\title{
Transcriptional control of the establishment of neocortical projections in the mammalian telencephalon
}

\author{
PhD Thesis \\ in partial fulfillment of the requirements \\ for the degree "Doctor of Phiosophy $(\mathrm{PhD}) /$ Dr. re.nat." \\ in the Neuroscience Program \\ at the Georg August University Göttingen, \\ Faculty of Biology
}

Submitted by

Swathi Srivatsa

born in

Mumbai, India

Göttingen, 2014

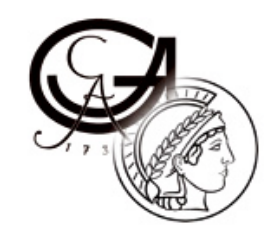




\section{DECLARATION}

This thesis has been written independently and with no other sources and aids than quoted.

Swathi Srivatsa

28 March 2014, Göttingen 


\section{TABLE OF CONTENTS}

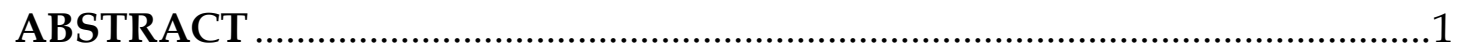

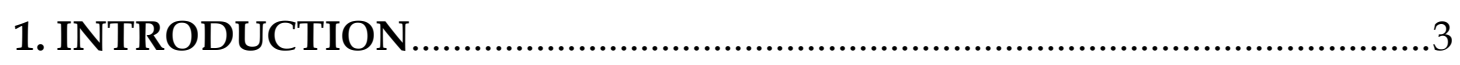

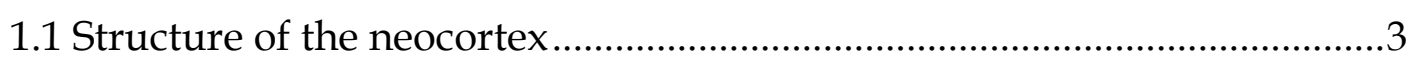

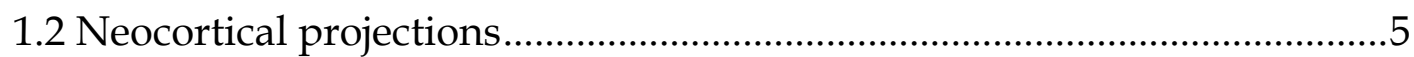

1.3 The molecular mechanism of axon guidance .......................................

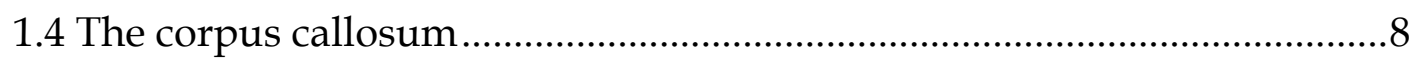

1.5 Role of Netrin1, Unc5C and DCC interactions in cortical axon guidance 9

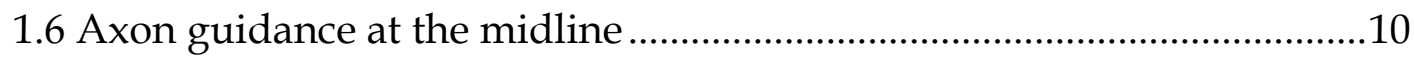

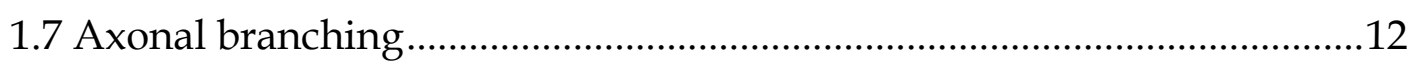

1.8 Microtubule assembly and function in neurons..................................... 14

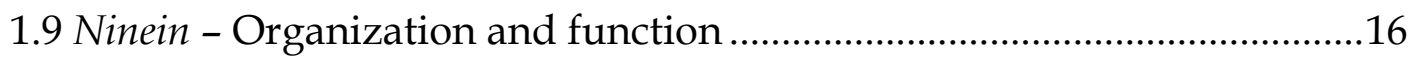

1.10 Transcriptional control over establishment of neocortical projections.17

1.11 Role of Satb2 and Ctip2 in controlling axonal trajectory choice .............18

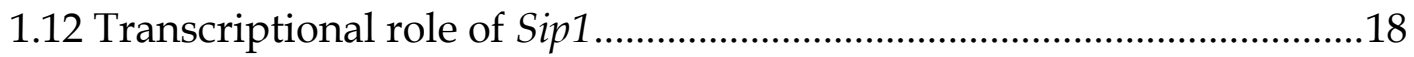

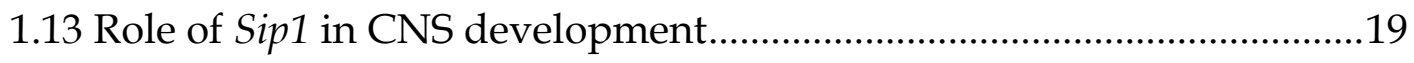

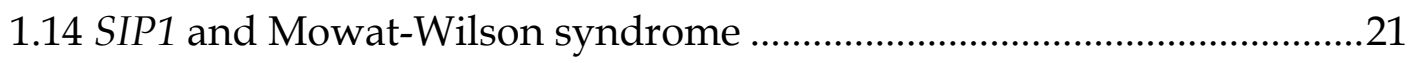

1.15 Scope of the study: Transcriptional role of Sip1, Satb2 and Ctip2 in axon

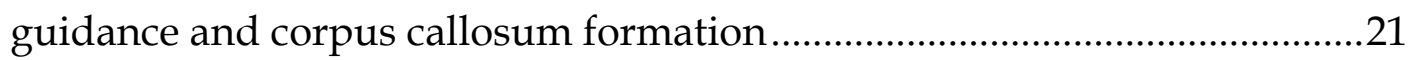

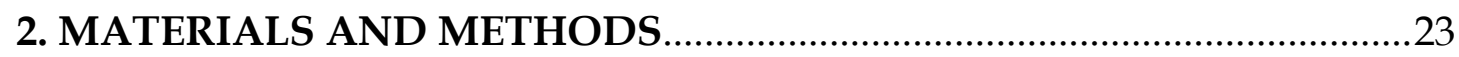

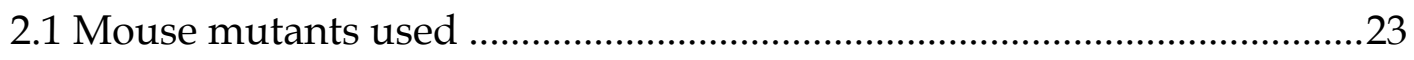

2.1.1 Sip1 conditional knockout ............................................................2 23

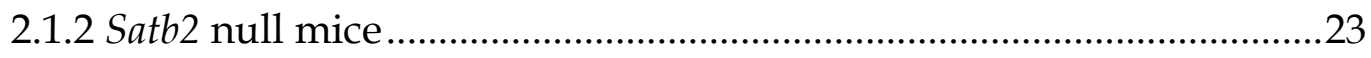

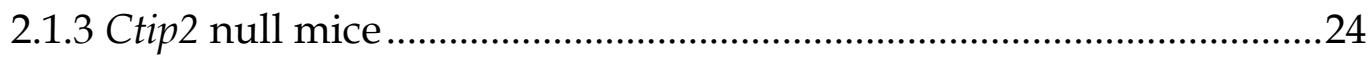

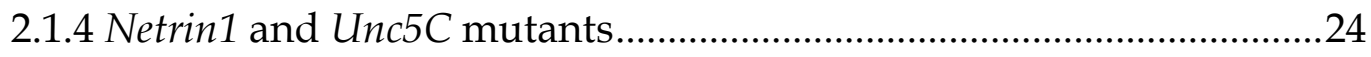

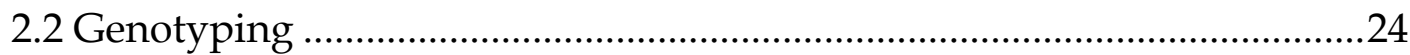

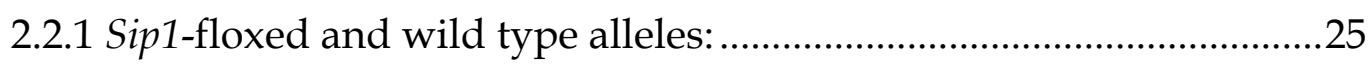

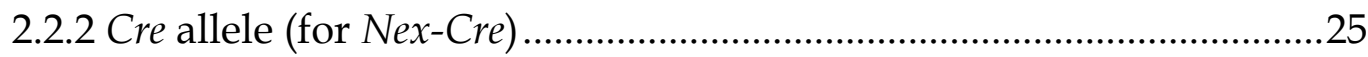

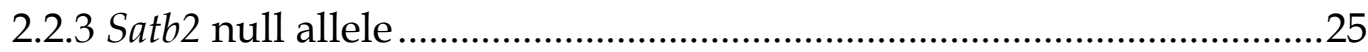




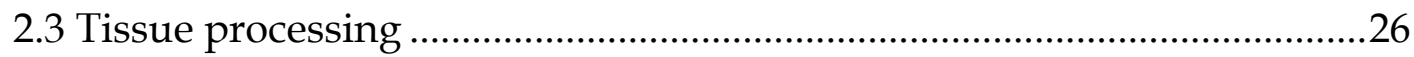

2.3.1 Embryonic and early postnatal tissue ....................................................26

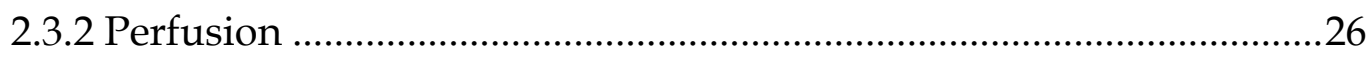

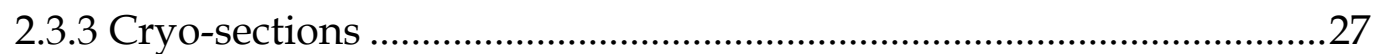

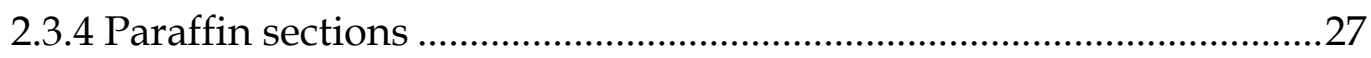

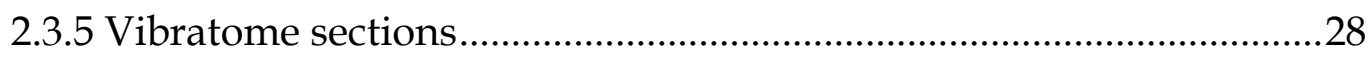

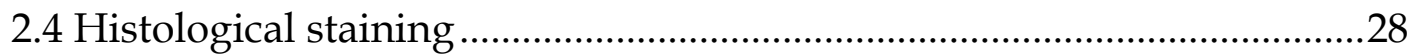

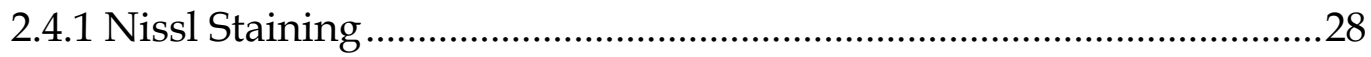

2.4.2 Immunohistochemistry using fluorescent primary and secondary antibodies 29

2.4.3 Immunohistochemistry using alkaline phosphatase based

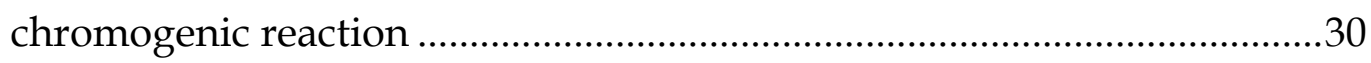

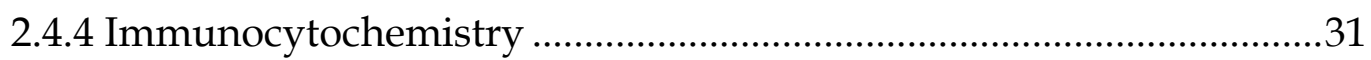

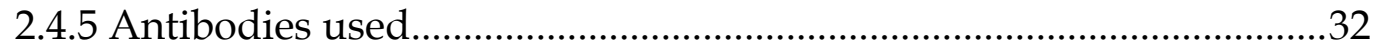

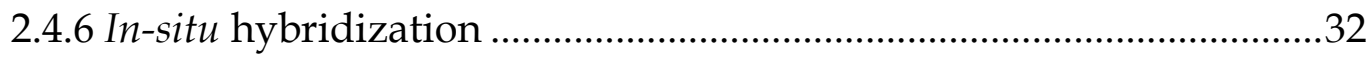

2.4.7 Immunofluorescence - Fluorescence in situ hybridization (IF-FISH)

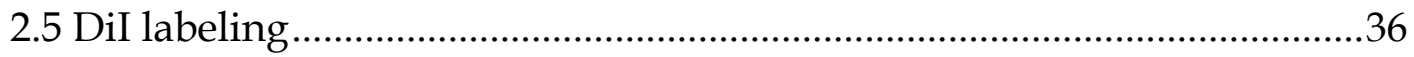

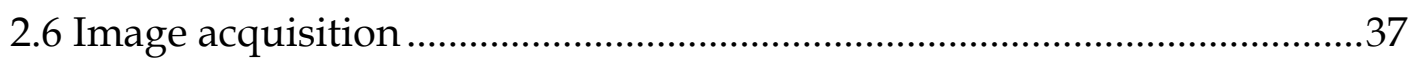

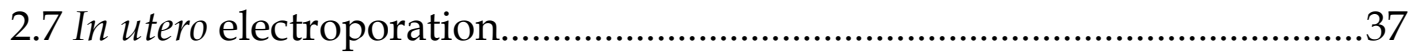

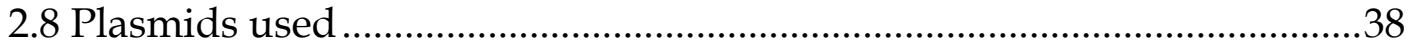

2.9 DCC knockdown assay using shRNA …………………...........................

2.10 Dissociated cortical neuron culture............................................................40

2.11 Nocodazole assay ....................................................................................

2.12 GFP-EB3 +TIPs live imaging ................................................................

2.13 In vitro cortical explant outgrowth assay ...................................................4

2.14 Organotypic slice culture and bead implantation assay ...........................43

2.15 Chromatin-Immunoprecipitation (ChIP) ..................................................4

2.16 Luciferase Assay .....................................................................................4

2.17 Data analysis and Statistics....................................................................4

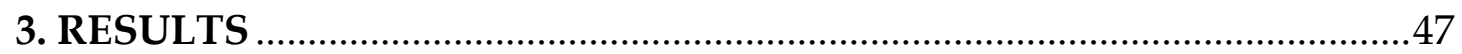


3.1 Commissural projections are severely altered in the Sip1 conditional knockout

3.2 Cortico-fugal projections are affected in the Sip1 conditional knockout 48 3.3 Retrograde DiI labeling reconfirms the absence of the corticospinal tract and the presence of the corticothalamic tract. 50

3.4 The corticospinal tract is present at early developmental stages in the Sip1 mutant 50

3.5 Loss of corticospinal tract is not a due to cell death of Layer V neurons51 3.6 Midline glia is almost completely lost in the Sip1 conditional mutant ...53 3.7 Subcallosal sling is not formed in the Sip1 conditional mutant 54

3.8 Slit1, Sema3C and other axon guidance molecules show altered expression levels in the Sip1 mutant.....

3.9 The lack of Sema3C expression in the Sip1 mutant is a non-cell autonomous effect. 59

3.10 Sip1 mutant shows altered midline morphology

3.11 Altered distribution of neurons at the midline is a non-cell autonomous effect of Sip1 deletion

3.12 Cell autonomous defects also contribute to the acallosal phenotype in the Sip1 mutant

3.13 Mosaic deletion of Sip1 is achieved by electroporation of Cre recombinase.... 65

3.14 Cell intrinsic deletion of Sip1 also affects CC formation 66

3.15 Callosally projecting neurons within the cingulate cortex are also affected by Sip1 deletion .68

3.16 Cell autonomous deletion of Sip1 slows the rate of axon extension 69

3.17 Sip1 deficiency affects ipsilateral axon-collateral formation 70

3.18 Microtubule associated protein - ninein, is downregulated in the Sip1 mutant. .73

3.19 Sip1 regulates ninein expression through direct binding .......................74

3.20 Sip1 binding to the ninein enhancer is functional .................................76

3.21 Ninein can be detected in the axons, axonal branches and the axonal growth cone of developing neurons 
3.22 Axonal growth is retarded in Sip1 deficient neurons in vitro 80

3.23 Restoration of ninein expression in Sip1 deficient neurons rescues the corpus callosum.

3.24 Restoration of ninein expression in Sip1 deficient neurons restores ipsilateral axonal branching of layer II and III projection neurons

3.25 Retarded microtubule growth contributes to the stunted growth of Sip1 deficient axons and is controlled by ninein

3.26 Ninein influences the stability of axonal microtubules... .86

3.27 Unc5C expression requires Satb2 and is restricted to callosal neurons.89

3.28 Ctip2 directly represses Unc5C expression .92

3.29 Partial Restoration of the CC in Satb2--;Ctip2-/ compound mutants .....96 $3.30 S a t b 2$ deficient callosal neurons have defects in axon targeting and not outgrowth or midline guidance. . .97

3.31 Restored expression of Unc5C in Satb2\% mutants results in partial rescue of $\mathrm{CC}$

3.32 Neocortical callosal axons are repelled by Netrin1 100

3.33 Misrouting of Satb2 positive axons in both Netrin1 and Unc5C mutants.

3.34 Satb2 is a direct repressor of DCC transcription. 104

3.35 Downregulation of DCC in Satb2 mutants results in partial rescue of the CC 108

3.36 Netrin1/DCC/Unc5C interactions regulate callosal projections of only deep layer neurons

3.37 Satb2 has limited capacity to restore the corpus callosum through cell intrinsic mechanisms

4. DISCUSSION

4.1 Commissural and sub-cortical projections are disrupted in the Sip1 mutant.

4.2 Dual role of Sip1 in CC formation

4.3 Non-cell autonomous role of Sip1 at the cortical midline. 
4.5 Mechanism involved in cell intrinsic control of Sip1 over axon growth

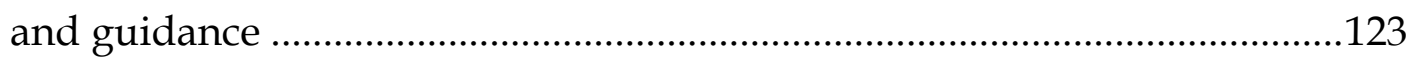

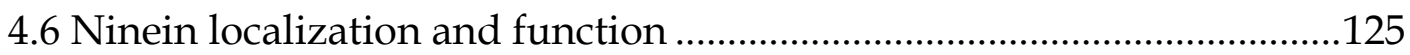

4.7 Mode of action: Ninein stabilizes microtubules .....................................126

4.8 Ninein function in axon collateral formation.........................................127

4.9 Microtubule stability influencing microtubule growth.........................128

4.10 New insights gained: The role of Sip1 in axon development ...............130

4.11 Satb2 and Ctip2 control the formation of divergent axonal paths........132

4.12 Lack of Ctip2 repression in the absence of Satb2 contributes to the acallosal phenotype in Satb2\% mice

$4.13 \mathrm{Unc5C}$ is under the direct transcriptional control of Ctip2 and indirect control of Satb2

4.14 Unc5C mutants also show corpus callosum defects

4.15 Unc5C aids in corpus callosum formation in response to Netrin1 expression

4.16 Unc5C and Netrin1 mutants show similar axonal behavior albeit dissimilar callosal phenotype

4.17 Together with Netrin1 - Unc5C interaction, Netrin1 - DCC interaction regulates medial vs. lateral axonal trajectory choice

4.18 Satb2-\%, DCC-/- and Netrin1 mutants show disparate accalosal phenotypes.

4.19 Cell intrinsic control of Satb2/Ctip2 and hence DCC/Unc5C in the formation of commissural vs. corticofugal projections is restricted to a suppopulation of deep layer neurons

4.20 New insights gained : Understanding the molecular basis of axonal trajectory choice. 


\section{LIST OF FIGURES}

Figure 1. Cortical projections are severely altered in the Sip1 mutant.................................................... 47

Figure 2. Commissural and corticofugal projections are affected in the Sip1 mutant.......................... 48

Figure 3. Corticospinal tract is present at birth (P0) in the Sip1 mutant.............................................. 51

Figure 4. No apparent cell death of Sip1 deficient layer V neurons....................................................... 52

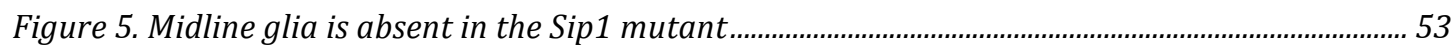

Figure 6. Calretinin positive sub-callosal sling neurons are mislocalized................................................. 55

Figure 7. Many axon guidance molecules including Slit1 and Sema3C show altered expression

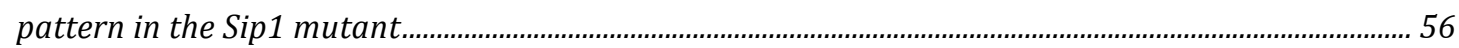

Figure 8. Loss of Sema3C expression in the Sip1 mutant is a non-cell autonomous effect..................... 59

Figure 9. Sip1 mutant midline is narrower and shows an altered distribution of cell density ............ 60

Figure 10. Change in relative distribution of cell density at the midline is a non-cell autonomous

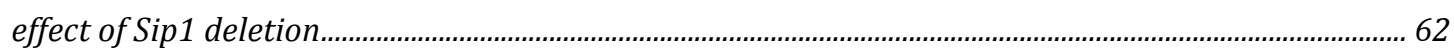

Figure 11. Acallosal phenotype in Sip1 mutant is due to both cell-intrinsic as well as cell-extrinsic

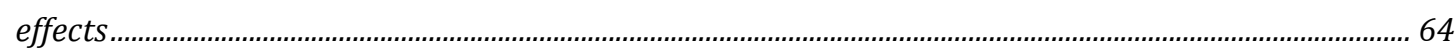

Figure 12. Cell autonomous deletion of Sip1 through in utero electroporation of Cre............................ 66

Figure 13. Callosal fibers fail to cross the midline on cell autonomous deletion of Sip1 ........................ 67

Figure 14. Projections of cingulate callosal axons are also affected due to cell intrinsic deletion of Sip1.

Figure 15. Lack of corpus callosum crossing with cell autonomous deletion of Sip1 is due to delay in axon extension 70

Figure 16. Upper layer neurons commence the formation of ipsilateral axonal collaterals at the level

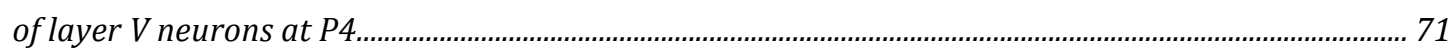

Figure 17. Sip1 deficient upper layer neurons do not form ipsilateral axon collaterals........................ 72

Figure 18. Ninein is downregulated in the Sip1 mutant cortex ….............................................................. 74

Figure 19. Sip1 directly binds to ninein promoter region and regulates its expression......................... 75

Figure 20. Luciferase assay confirms functional binding of Sip1 to ninein upstream region............... 76

Figure 21. Ninein localizes to the soma, dendrites, axon and axonal growth cone................................. 78

Figure 22. Ninein localizes to axonal and dendritic branches in neurons.................................................. 79

Figure 23. Sip1 negative neurons show stunted axonal growth in vitro, which is rescued by restoring ninein expression 80

Figure 24. Restoring ninein expression partially rescues the corpus callosum in brains with cell autonomous deletion of Sip1.

Figure 25. Axon collateral formation by upper layer neurons is restored by expression of ninein in Sip1 deficient neurons

Figure 26. Microtubules grow slower in Sip1 deficient cells and the growth rate is restored in the presence of ninein.

Figure 27. Ninein stabilizes microtubules in the axons. 
Figure 28. Unc5C is expressed by callosally projecting Satb2 positive neurons and Satb2 regulates

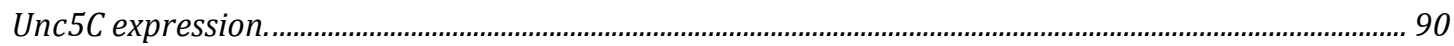

Figure 29. Ctip2 is up-regulated in the deep layers of the cortex upon Satb2 deletion.......................... 92

Figure 30. Unc5C expression is restored in Satb2\%;Ctip2\% double mutants ........................................... 93

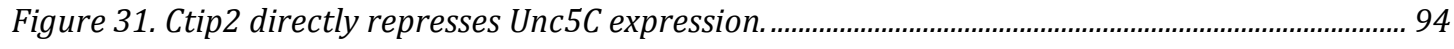

Figure 32. The corpus callosum is partially restored in Satb2\%; Ctip2\% compound mutants .............. 96

Figure 33. Satb2\% neurons do not show defects in neurite outgrowth and midline guidepost cells are not altered in Satb2\%, Ctip2\% or Satb2\%Ctip2 \% mutants........................................................................ 98

Figure 34. The corpus callosum is partially restored by over-expressing Unc5C in Satb2\% mutant cortex.

Figure 35. Netrin1 repels Unc5C positive neocortical axons. 101

Figure 36. Satb2 cells are similarly misrouted to subcortical targets in Netrin1 and Unc5C mutants

Figure 37. DCC is up-regulated in the Satb2\% mutant and Satb2\%;Ctip2\% double mutant cortex.105 Figure 38. Satb2 is a direct transcriptional repressor of DCC expression

Figure 39. Down-regulating DCC in the Satb2\% cortex leads to a partial rescue of the corpus callosum.

Figure 40. Over-expression of Unc5C with the simultaneous down-regulation of DCC does not rescue the corpus callosum 110

Figure 41. Over-expression of Unc5C or down-regulation of DCC at E14.5 does not rescue the CC in

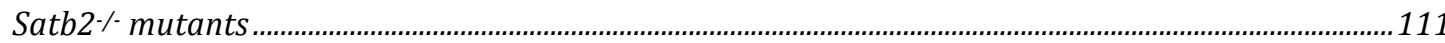

Figure 42. Satb2 electroporation in Satb2\% cortex partially rescues the corpus callosum. 113

Figure 43. Quantification of CC with respect to efficiency of electroporation in wild type and Satb2\% mutant.

Figure 44. Connectivity defects observed in the Sip1 mutant forebrain 118

Figure 45. Non-cell autonomous defects in the Sip1 mutant midline. 121

Figure 46. Ninein localizes to neocortical axons and influences microtubule growth and stability downstream of Sip1 130

Figure 47. Satb2 Ctip2 dependent establishment of cortical connections 132

\section{LIST OF TABLES}

Table 1. Axon guidance molecules tested by ISH in the Sip1 mutant. 58 


\begin{abstract}
Neuronal connections in the neocortex that relay and integrate information form the basis of our higher cognitive abilities. Transcription factors play an important role in controlling the trajectory of neuronal projections. They act as molecular switches capable of modulating downstream targets that in turn alter growth cone behavior. In this study we have investigated the role of three different transcription factors, namely Sip1, Satb2 and Ctip2 and their downstream mechanisms that influence the establishment of neocortical projections.
\end{abstract}

Sip1 is an important transcription factor that regulates many different aspects of CNS development. We show that, Sip1 also plays an important role in the development of neocortical projections. In the absence of Sip1 from the neocortex, many axonal tracts within the forebrain are severely affected. While the commissural projections like the corpus callosum (CC) and anterior commissure are not formed in the Sip1 mutant, corticofugal connections like the cortico-spinal tract is also affected.

Sip1 exerts both cell autonomous as well as non-cell autonomous control over CC formation. The non-cell autonomous effects of Sip1 deletion alter the cortical midline, making it unfavorable for commissural axons to cross. Fusion of the dorsal midline does not occur, different populations of GFAP positive midline glia are either reduced or absent and subcallosal sling neurons are mislocalized.

Cell autonomous deletion of Sip1 also results in the lack of corpus callosum formation and lack of ipsilateral axon-collateral formation. Sip1 mediates these effects through its direct downstream effector ninein, a microtubule binding protein. We further show that ninein in turn influences axon growth and branching by affecting microtubule stability and dynamics. 
The transcription factors Satb2 and Ctip2, are critical regulators of neuronal cell fate that control commissural versus corticofugal projections respectively. In this study, we have investigated the axon guidance molecules downstream of Satb2 and Ctip2 that govern the trajectory choice made by neocortical neurons. We show that Satb2 and Ctip2 transcriptionally regulate the expression of two Netrin1 receptors - DCC and Unc5C respectively. Within deep layer neurons, Netrin1- Unc5C/DCC interactions are involved in controlling the commissural versus corticofugal trajectory choice made by these axons. 


\section{INTRODUCTION}

The neocortex, which forms the outer most cover of the brain, is arguably the crowning achievement of mammalian evolution (Hill \& Walsh, 2005). It forms the biological substrate of our higher cognitive capacity that includes language, problem solving, reasoning, decision making etc., abilities that distinguish humans from other less-evolved species. As a first step in understanding these processes and how the brain computes seemingly complex tasks with tremendous ease, one must first understand the development of the neocortex (Geschwind \& Rakic, 2013).

\subsection{Structure of the neocortex}

The mammalian neocortex is a highly organized structure comprising of a vast array of neurons that have different cellular identity, structure and function (Fishell \& Hanashima, 2008a). This includes projection neurons, that extend their axons much further than their point of origin and are responsible for relaying information both within the cortex and to other sub-cortical structures, and interneurons that project their axons more locally and are involved in local circuitry. They have a modulatory function. The neocortex arises from a relatively smaller population of precursors or progenitors called radial glial cells that line the dorsal lining of the lateral ventricles called the ventricular zone (VZ) (Anthony et al., 2004; Malatesta, Hartfuss, \& Götz, 2000a, 2000b; McConnell, 1995; Noctor et al., 2001; Noctor et al., 2002). Apart from producing neurons and glia, the radial glial cells also known as the apical progenitors give rise to basal progenitors that occupy the subventricular zone (SVZ). The basal progenitors act as an additional step of amplification during neurogenesis. Sequential symmetric and asymmetric divisions of the progenitors give rise to the diverse set of projection neurons and glia that populate the neocortex (Fishell \& Kriegstein, 2003; Miyata et al., 2001; Noctor et al., 2004). Within the cortex the projection neurons are 
arranged in six different layers. The neocortex shows a characteristic insideout manner of generation wherein the first neuronal layer produced (layer VI) occupies the inner most region of the cortex and every subsequent layer produced, then occupies the next higher position. Neurons occupying the deeper layers of the neocortex are generated earlier by apical progenitors and the neurons occupying the upper layers of the cortex are born later from basal or intermediate progenitors (Desai \& McConnell, 2000; Frantz \& McConnell, 1996; Haubensak et al., 2004; Molyneaux et al., 2007; Rakic, 1974; Takahashi, 1995; Tarabykin et al., 2001). Every neuron, cell intrinsically, possesses the ability to respond to the immediate cellular environment to migrate through the cortex, passing previously born neurons and occupying its respective position within the cortical plate (Angevine \& Sidman, 1961; Huang, 2009; Kriegstein \& Noctor, 2004a, 2004b; Nadarajah et al., 2001; Nadarajah \& Parnavelas, 2002). Within a layer, neurons share to a large extent a common morphology, function and molecular identity (Fishell \& Hanashima, 2008b; Greig et al., 2013; Guo et al., 2013; Kmet et al., 2013; Rouaux \& Arlotta, 2013).

- Layer VI is comprised of the corticothalamic projection neurons that connect different regions of the cortex to specific nuclei within the thalamus.

- Layer $\mathbf{V}$ is comprised of pyramidal neurons that extend their axons all the way to the spinal cord relaying information out of the brain to subcerebral structures and hence are called the corticospinal neurons. These neurons also project to the optic tectum, striatum and pons. Layer $\mathrm{V}$ and $\mathrm{VI}$ are together referred to as deep layer (DL) neurons.

- Layer IV neurons also referred to as spiny-stellate neurons because of their morphology, play the critical function of receiving inputs from the thalamus. Hence axons of the thalamic neurons that convey information to the cortex innervate layer IV.

- Layer III and II comprise of projection neurons that primarily project within the cortex either within the same hemisphere or to the other hemisphere hence conveying and integrating information from 
different parts of the cortex. Together they are referred to as upper layer neurons.

- Layer I is also called the molecular layer and contains the apical dendrites of the neocortical neurons and a population of cells called the Cajal retzius cells.

\subsection{Neocortical projections}

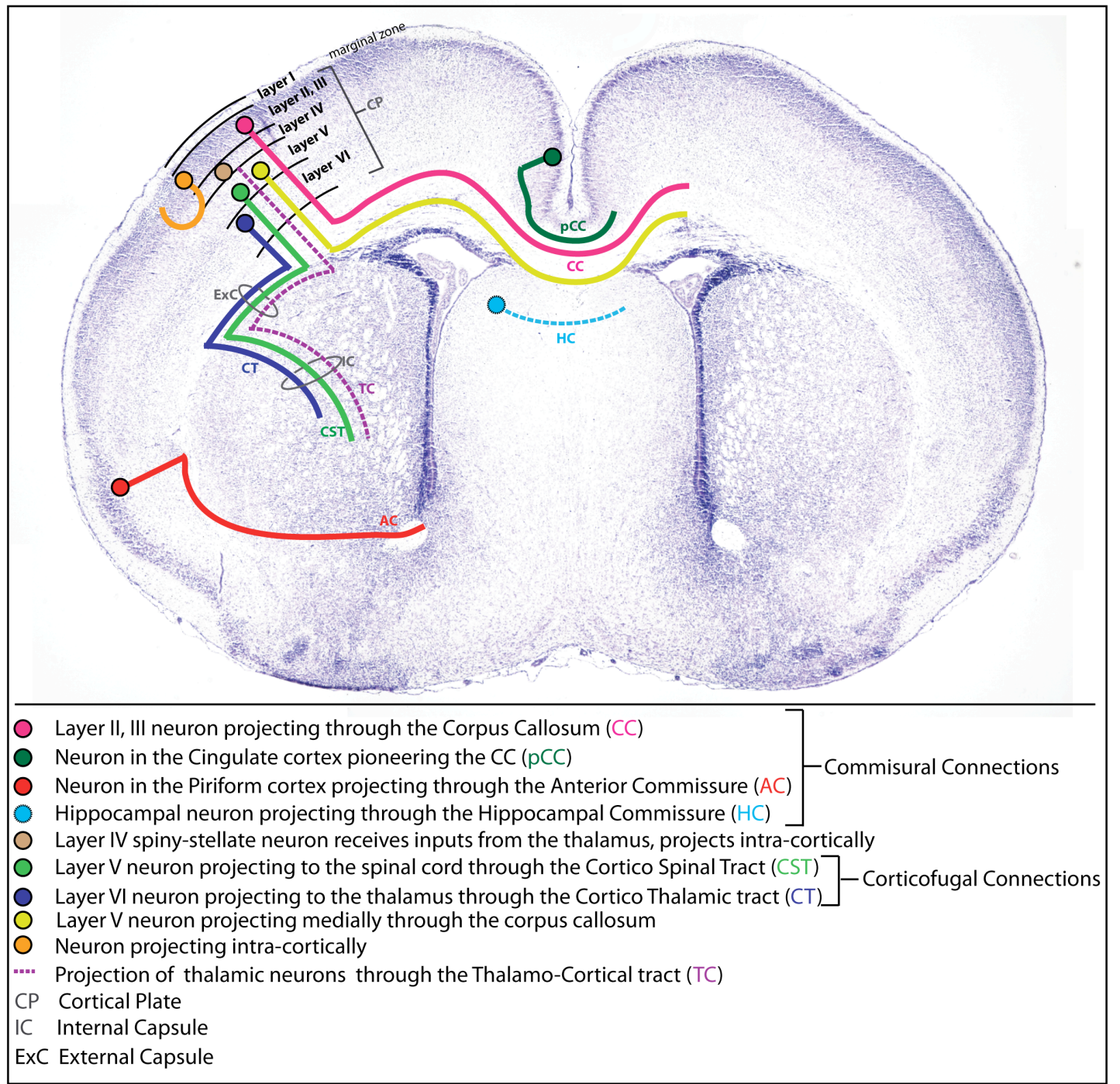

Figure (i). Major axonal pathways of the forebrain.

The figure shows a schematic representation of the major axonal tracts within the forebrain. Commissural projections that connect the two hemispheres with each other consist of the Corpus callosum (CC), Hippocampal commissure (HC) and Anterior commissure (AC). The corticofugal projections that connect cortical neurons to subcortical targets comprises of the Corticothalamic tract (CT) and Corticospinal tract (CST). 
As neurons migrate to their final position within the different layers of the cortex they simultaneously project their axons to different targets (Noctor et al., 2004). The projections that arise from the different layers of the cortex fasciculate into axonal bundles forming the white matter of the brain (Innocenti \& Price, 2005). Based on where they originate and where they project to, they can be broadly classified as

- Cortico-cortical connections

- Cortico-fugal connections

Cortico-cortical projections comprise of the intra-hemispheric projections, which connect neurons within the same hemisphere and inter-hemispheric commissural projections that connect one neocortical hemisphere with the other. The commissural projections of the cortex include the corpus callosum (CC) and the anterior commissure (AC). In the mouse, mainly axons from layer II and layer III and to a lesser extent from layer V project through the commissural path (Aboitiz \& Montiel, 2003; Fame, MacDonald, \& Macklis, 2011; Robin, 2006; Schüz \& Preiß1, 1996). The corticofugal projections are formed by axons that leave the neocortex and project to various subcerebral structures. These include the corticothalamic tract (CT) and the corticospinal tract (CST). Projections to other structures like the midbrain, pons etc. emanate as branches from the CST during development and are pruned when synaptic targets are recognized (Aronoff et al., 2010; Fame et al., 2011; Kennedy \& Dehay, 1993; Molnár et al., 2007).

Apart from these projections that arise from within the neocortex, the thalamocortical axons (TC) project from the thalamus into the neocortex and the hippocampal commissure (HC) interconnect the two hippocampi. Since all of these cortical projections act as conduits for the passage of information to and from the cerebral cortex to other regions of the central nervous system, understanding how these connections are established forms an integral part of understanding brain function (Innocenti \& Price, 2005). 


\subsection{The molecular mechanism of axon guidance}

Observations of early development of axonal trajectory in vivo have revealed that the process of path finding is highly directed, precise and stereotyped. The axonal growth cone extends towards the target making apparently very few errors on the way. Axons sense a variety of molecules present in their immediate environment that help direct their trajectory. The axon in turn carries the machinery that responds to these guidance cues by extension, growth cone collapse or axonal turning. Apart from molecular-cue based axon guidance there also exists activity dependent axon guidance, which helps in axonal pruning and establishing the right synaptic contacts. Hence, molecular-cue based axon guidance seems to be an initial mode of navigation adopted by the axon for target finding and the activity dependent mode is involved in pruning these connections (Goodman \& Shatz, 1993; TessierLavigne \& Goodman, 1996).

Work from over three decades in the field of axon guidance and path finding has led to the discovery of a plethora of ligands and their cognate receptors, which act as guidance cues and help in directing the axon towards its target and away from non-target locations. The guidance cues comprise of molecules that are secreted (and hence act over long-range) or membrane bound (acting over a short- range). They are also classified as chemo/contact attractant or repellent based on the response that they illicit. These ligandreceptor pairs among others include Netrins - UNC5s/DCC, Robo-Slit, Semaphorins - Plexins and Neuropillins, Ephrins and Eph-receptors (Bagnard et al., 1998; Brose \& Tessier-Lavigne, 2000; Kennedy et al., 1994; Kidd et al., 1998; Killeen \& Sybingco, 2008; Kullander \& Klein, 2002; Long et al., 2004; Nakamura, Kalb, \& Strittmatter, 2000; Seeger et al., 1993; Serafini et al., 1996). Although one set of ligand - receptor pair might elicit one kind of response from an axon, it is a combination of these attractive and repulsive forces that defines the trajectory of the axon. 
Furthermore, it is very interesting to see how the mechanism of guiding axons to their target has evolved so as to make the process more precise and time efficient. For example, many structures called 'guideposts' can be found within the axonal trajectories that act as intermediate targets. Axons are hence able to traverse long distances with relative ease as they respond to these intermediate cues that breakdown the entire journey of a neuron into smaller parts (Colón-Ramos \& Shen, 2008). For example, commissural axons found in the brain and the spinal cord respond to groups of specialized cells present in the midline that are essential for directing the axons to the contralateral side (Colamarino \& Tessier-Lavigne, 1995b; Klämbt, Jacobs, \& Goodman, 1991). Another beneficial mechanism that neurons have adopted is the 'pioneer and follower' mechanism (McConnell, Ghosh, \& Shatz, 1989). Pioneer axons are the very first set of axons that project from the place of origin towards the target. These show a much slower rate of growth and more elaborate growthcone structure and dynamics. The follower axons fasciculate with the pioneers and hence show relatively simpler growth cone structure and extend much faster. If these axons need to deviate from the path they can in some cases develop the characteristics of a pioneer by elaborating their growth cone and consequently slowing down their growth rate (Bak \& Fraser, 2003; Kim et al, 1991).

\subsection{The corpus callosum}

The corpus callosum (CC) is the largest axonal tract in the brain and consequently the most important commissural connection. It comprises of about 80 percent of the commissural fibers in the brain and helps in the coordinated exchange of information between the two cerebral hemispheres. In the mouse, a majority of the axons forming the CC originate in layer II and III, but few neurons of layer $\mathrm{V}$ also project medially through the corpus callosum (Aboitiz \& Montiel, 2003; Fame et al., 2011; Robin, 2006; Schüz \& Preißl, 1996). Partial or complete loss of the corpus callosum is called agenesis of the corpus callosum (ACC) and is associated with over 50 human 
pathologies, suggesting that defects in a variety of developmental paradigms ranging from cell proliferation, migration to failure in axon guidance can results in abnormalities in this structure (Paul et al., 2007; Richards, Plachez, \& Ren, 2004). Individuals with ACC present a range of behavioral as well as neuropsychiatric abnormalities such as learning deficits, sleep disorders, language and social communication disorders. The CC is pioneered by axonal projections of neurons from the medial most part of the cortex called the cingulate cortex (deAzevedo et al., 1997; Koester \& O'Leary, 1994). The first set of fibers seen crossing the midline in case of the mouse is seen as early as E16.5, following which callosal axons from other regions of the cortex fasciculate and form the thick commissural bundle (Rash \& Richards, 2001).

\subsection{Role of Netrin1, Unc5C and DCC interactions in cortical axon}

\section{guidance}

Netrin1, the first identified diffusible long-range guidance cue, is expressed at the ventral midline of the hindbrain and spinal cord as well as ventral structures of the forebrain (Colamarino \& Tessier-Lavigne, 1995; Kennedy et al., 1994; Métin et al., 1997; Serafini et al., 1996). It mediates both chemoattractive as well as chemorepulsive effects based on the receptor(s) expressed on the axonal growth cone (Colamarino \& Tessier-Lavigne, 1995a). DCC is a receptor of Netrin1 and mediates an attractive response on binding to Netrin1 (Deiner et al., 1997; Keino-Masu et al., 1996). Unc5C is another known receptor of Netrin1 (Ackerman et al., 1997). Unc5C, either independently or together with DCC, mediates a repulsive response to Netrin1 signals (Chan et al., 1996; Finger et al., 2002; Gitai et al., 2003; Hong et al., 1999; Kim \& Ackerman, 2011). In the forebrain, Netrin 1 is expressed in the ventral telencephalon within the ganglionic eminence, which gives rise to the basal ganglia (Hamasaki et al., 2001; Métin et al., 1997). It is also expressed in the ventral region of the dorsal midline during early embryonic stages till around E15.5 (Barallobre et al., 2000; T Fothergill et al., 2013). Unc5C and DCC, on the other hand, are expressed by cortical projection neurons. DCC is 
more strongly expressed by neurons present in the cingulate cortex and Unc5C shows a stronger expression within the lateral cortex. The interaction between these molecules is crucial for CC development. While Netrin1 and DCC mutant brains lack a corpus callosum (Fazeli et al., 1997; Ren et al., 2007; Serafini et al., 1996), Unc5C deficient brains show the presence of callosal crossing (Finger et al., 2002).

\subsection{Axon guidance at the midline}

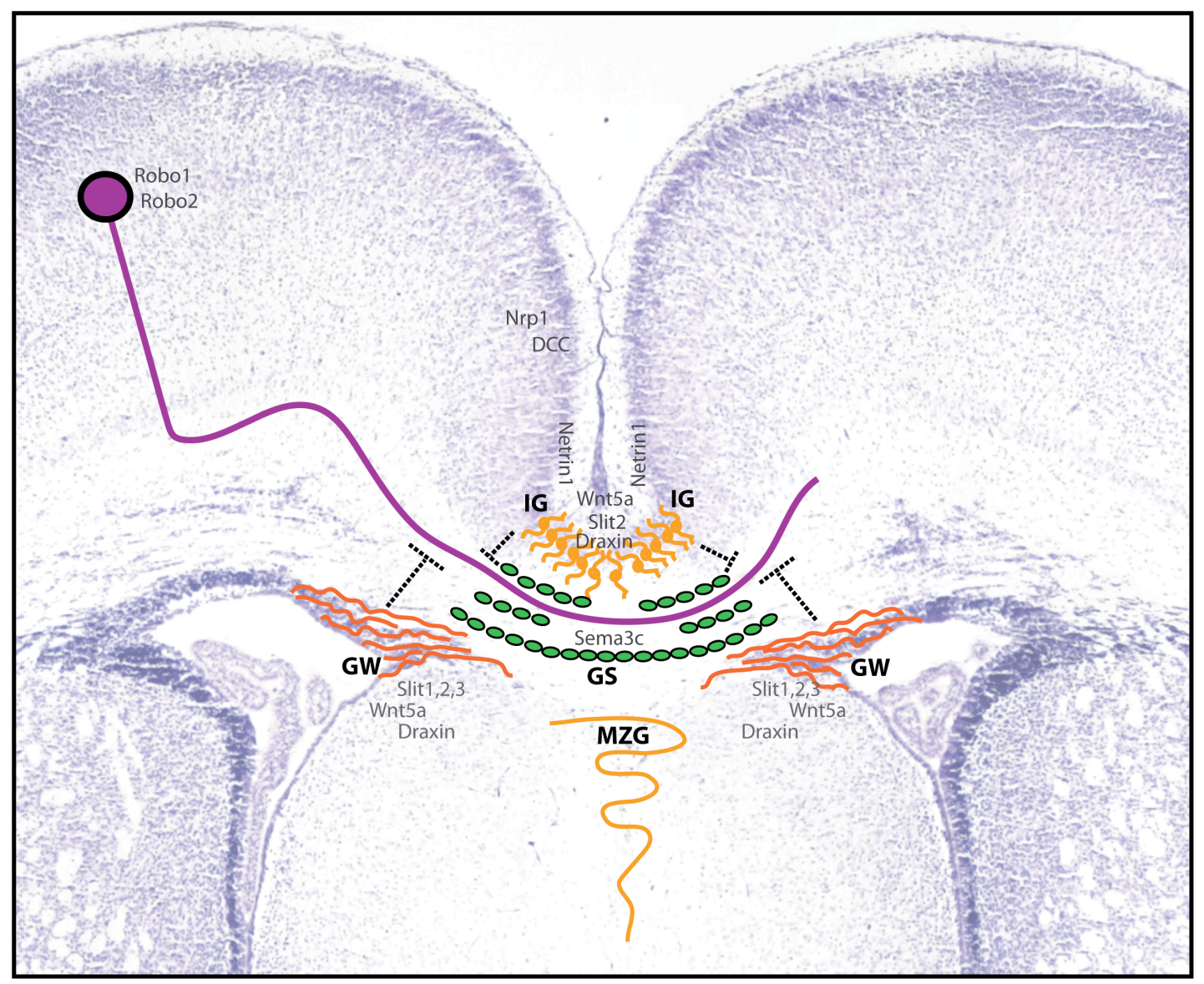

Figure (ii). Midline guidance structures that aid in corpus callosum formation

The figure shows a schematic representation of the midline glia as well as the sub callosal sling neurons that act as guidepost cells for the formation of the corpus callosum. The indusium griseum (IG), glial wedge cells $(\mathrm{GW})$ and the midline zipper glia (MZG) form the midline glia that express different guidance cues like Slit 1, 2, 3, Wnt5a, Draxins etc. The subcallosal sling neurons, which are a mixed population of glia, glutamatergic and GABAergic cells also act as accessory guidance structures. A population of sling neurons expresses Sema3C. The cortical neurons in turn express cognate receptors or respective ligands that make them responsive to these midline cues, for example Robo1 and Robo2. 
It has been shown that the midline of the central nervous system is a very critical organizing center with respect to axon guidance and establishment of commissural connections (Colamarino \& Tessier-Lavigne, 1995b; Dickson \& Zou, 2010; Evans \& Bashaw, 2010; Kaprielian, Imondi, \& Runko, 2000). Morphologically the dorsal midline has to fuse in order to allow for axons to navigate on a permissible substrate. At early embryonic stages when the birth of callosal neurons has commenced (E14.5- E15.5), the cerebral hemispheres are fused ventrally. Dorsally, they are separated mid-sagittally by a connective tissue filled fissure. The process of midline fusion has been suggested to involve the fusion of the basal lamina while simultaneously eliminating the fibroblast from the fissure, which is then replaced by neural tissue. This process is though to be carried out by glial cells found in the midline that essentially 'zips up' the cortical midline. This population is hence termed as the midline zipper glia (MZG) (Silver et al, 1993).

The cortical midline, apart from acting as a substrate for axons to pass through, also acts as a locus that expresses a variety of physical and molecular guidance cues. These cues mediate a delicate balance between attractive forces, which help in bringing the axons from one hemisphere towards the midline, and repulsive forces, that allow these axons to leave the vicinity of the midline and move towards their target on the contralateral side. These signals have to be appropriately regulated to prevent the already crossed axons from re-entering the midline and re-crossing towards the ipsilateral cortex (Chen et al, 2008; Richards et al., 2004; Sabatier et al., 2004). The accessory midline structures that aid in CC formation can be grouped into two different populations. The first group of cells is the midline glia, which includes the glial wedge (GW) and the indusium griseum glia (IG), and the second group of cells is called the midline sling neurons. The GW cells arise from the dorso-medial edge of the lateral ventricle and are diffrentiated radial glial progenitors. They are GFAP positive and are present as wedge shaped structures in the ventro-lateral aspect of the CC crossing. The IG is also GFAP positive but has been proposed to originate from the midline zipper glia. It is 
positioned dorsal to the CC crossing. Both these glial populations have been shown to express slit-2 and hence repel Robo-1 and Robo-2 expressing cortical axons in vitro. It is hence thought that the midline glial structures help in axonal turning of the $\mathrm{CC}$ at the midline and restricts the pathway of these axons so that they form the characteristic U-shaped turn (T Shu \& Richards, 2001). For example, it has been shown that if the midline glial structure are severed, the CC fails to cross the midline and instead forms Probst bundles on either sides of the midline (Silver \& Ogawa, 1983).

The midline glial population act in concert with the subcallosal sling neurons. The sling consists of immature neurons born between E15.5 and P2 in the dorsal SVZ that migrate towards the midline and occupy the ventral aspects of the CC. They have been found to express neuronal markers like NeuN and calretinin (Shu et al, 2003). They also play an important role in CC guidance as they express Sema3C that functions as an attractant molecule for the pioneering axons expressing Nrp1. Apart from the glutamatergic neurons, GABAergic interneurons are also found within the CC white matter as a part of the subcallosal sling (Niquille et al., 2009).

\subsection{Axonal branching}

Axons form the principal output structure of neurons as they transduce electrical impulses from the cell body to the synapse. In order for an individual neuron to connect to multiple targets the primary axon of the neuron frequently branches into collaterals. Axonal branch formation is a very dynamic process, which involves rapid extension and retraction of axonal projections resulting in a net enlargement of arborization. Three different processes have been used to describe branch formation (Acebes \& Ferrús, 2000; Portera-Cailliau et al., 2005; Schmidt \& Rathjen, 2010);

- Splitting mode, where the growth cone bifurcates and the two growth cones extend in divergent directions. 
- Delayed mode, where a region of the axon with unstable cytoskeletal structure and filopodial activity is left in the wake of a growth cone, and an axonal branch sprouts from that point soon after the primary growth cone extends further.

- Interstitial mode, where axon collaterals emerge as a single outgrowth of the main axon shaft orthogonal to the primary axon. The branch initiation can take place days after the growth cone has passed the corresponding area (O'Leary \& Terashima, 1988; Szebenyi et al., 1998; Yamamoto et al., 1997).

Delayed interstitial branching is not a very well understood mechanism and has garnered a lot of interest over the years. One possible mechanism proposed for collateral branching from specific regions of the axon refers to target derived signals that induce branching behavior in axons (Kennedy \& Tessier-Lavigne, 1995) . Alternatively, it has also been shown that the axonal growth cone recognizes the target and exhibits a pausing behavior that involves extension, collapse and retraction in this region during axon extension. This growth cone behavior reorganizes the cytoskeleton in this region and demarcates the axonal position for future branch formation (Szebenyi et al., 1998).

Within the neocortex, the different classes of neurons have a characteristic axonal and dendritic branching pattern specific to the neuronal subtype and function. For example axons of layer II and III neurons extensively ramify within the layer and the descending axon also ramifies at layer $\mathrm{V}$. The axon passes through layer IV without branching (Burkhalter, 1989; Kritzer \& Goldman-Rakic, 1995; Thomson \& Lamy, 2007). Axon guidance molecules that are implicated in influencing axonal trajectories have also shown to be involved in initiating axonal collateral formation (Harwell et al., 2012; Heffner, Lumsden, \& O’Leary, 1990; Kennedy \& Tessier-Lavigne, 1995; Leary, 1996). These guidance signals elicit molecular pathways within the neuron that finally translates into cytoskeletal modifications. Branch formation is 
initiated by modulation of actin cytoskeleton and is then stabilized when a more rigid microtubule structure is formed.

\section{$\underline{1.8 \text { Microtubule assembly and function in neurons }}$}

In responses to the different guidance cues discussed above, on ligand activation the receptors on the growing axon elicit downstream signaling cascades, which finally converge in remodeling the axonal cytoskeleton. These cytoskeletal elements are the ultimate effectors of movement and guidance (Dent, Gupton, \& Gertler, 2011). The cytoskeleton of a cell acts as a scaffold that decides the shape of the cell, organization of its inner organelles and to a large extent the functioning of the cell. The cytoskeletal polymers are primarily actin-filaments and microtubules (MTs). MTs are tubular macromolecules formed by polymerized tubulin dimmers namely $\alpha$ - and $B$ tubulin. MTs have a distinct polarity that governs their biological function. The slow-growing minus-end corresponds to the end of the MT which has an $\alpha$ - subunit exposed and the fast-growing plus-end corresponds to the region with the B- subunit is exposed (Baas \& Ahmad, 1992). A characteristic property of MTs is that it exists in a state of dynamic instability, i.e. a constant state of growth (polymerization) and breakdown (catastrophe). This intrinsic property of MTs allows it to rapidly reorganize within a cell thus modifying the cell with respect to its environment or generate a pushing and pulling forces with in a cell, which is essential for processes like cellular migration and growth (Inoué \& Salmon, 1995; Kirschner \& Mitchison, 1986). The centrosome is the cellular organelle predominantly associated with nucleation or birth of MTs. More generically it is referred to as the microtubule organizing center (MTOC) which includes the pair of centrioles surrounded by the centrosomal matrix, also called the pericentriolar material (PCM) from which MTs emanate (Kellogg, Moritz, \& Alberts, 1994). In a neuron MTs are found throughout the cytoplasm and hence can be seen within the soma, axon and dendrites. MTs also show specialized organization within these structures. Within an axon the MTs are arranged such that all the minus-ends 
are towards the soma and the plus-end or the growing end is directed towards the growth cone (Heidemann, Landers, \& Hamborg, 1981). In case of dendrites, the MTs show mixed polarity (Baas, 1998; Keating et al., 1997). Although MTs are generated or nucleated at the centrosome they are released from the centrosome by MT cleaving enzymes like katanin and are then transported into the neurites by MT associated motor proteins like dynein. (Ahmad et al., 1999; Yu et al., 1993). These free MTs are essential for neurons to carry out their specialized function (Keating \& Borisy, 1999). For example, since the length of an axon has been found to vary from a few millimeters to more than a meter long, a single MT molecule for the entire length of the axon would not provide stability or functionality to the axon. Hence a series of short overlapping microtubules can be found in the axon that help as tracks for the cargo carrying machinery in addition to acting as structural scaffolds (Ahmad \& Baas, 1995; Baas \& Yu, 1996; Bray \& Bunge, 1981).

MTs are essential not only for polarizing neurons and for axonal and dendritic growth but also for neurite branching. It has been shown that a large degree of microtubule reorganization occurs at interstitial branch point of axons and dendrites (Dent et al., 1999). It has been suggested that similar to generating free microtubules in the axon, severing enzymes like katanin and spastin are involved in fragmenting microtubules within an axon, which are then transported into the developing axonal branches with the help of motor proteins such as dynein (Dent et al., 1999; Yu et al., 1994; Yu et al., 2008). Although a plethora of proteins that behave as MT associated proteins or MAPs have been discovered, not all of them have been assigned to a physiological or molecular function with respect to axonogenesis. Different MAPs have been shown to help in the assembly, growth or maintenance of the dynamic state of MTs (Conde \& Cáceres, 2009). MT capping proteins form one class of MAPs. These bind to the minus-end of MTs and assist in nucleation and/or provide stability to the growing MT (Keating \& Borisy, 1999). One such molecule that we have studied in the work presented here is ninein. 


\subsection{Ninein - Organization and function}

Ninein, a $235 \mathrm{kDa}$ coil-coil protein, was discovered as a centrosome associated protein localized to the pericentriolar matrix of the MTOC. It was shown to be associated with the centrosome throughout cell cycle and with mitotic spindles. It consists of a putative GTP binding site, four leucine zipper domains and a potential EF-hand-like domain and may exist in macromolecular complexes (Bouckson-Castaing et al., 1996). Interestingly, the human isoform of ninein has been shown to interact with the phosphorylating enzyme GSK3-ß (Y. R. Hong et al., 2000), which has shown to be extensively involved in microtubule assembly and dynamics (Galjart, 2005; Hur et al., 2011; Kuijpers \& Hoogenraad, 2011; Neukirchen \& Bradke, 2011; Sakakibara et al., 2013; Schmidt \& Rathjen, 2010). It has been suggested that the MTOC houses two distinct set of protein complexes, one that is involved in MT nucleation and the other that is involved in MT capping, anchoring and stabilization (Bornens, 2002; Mogensen et al., 2000; Mogensen, 1999). In cells that present the classical radial microtubule array, ninein was found to primarily localize to the mother centriole, which forms the center from where the radial microtubules emanate. Although, only the mother centriole has been shown to contain ninein, both the centrioles posses the same capacity to nucleate microtubules (Piel et al., 2000). Additionally, in polarized cells like the cochlear epithelial cells, where non-centrosomal microtubules are found, ninein was also shown to be associated with the minus-end of these MTs (Mogensen et al., 2000). $\gamma$-tubulin and pericentrin, molecules important for MT nucleation have been shown to be absent from these non-centrosomal MTs. It has hence been suggested that ninein plays a role in stabilizing, positioning and anchoring the minus-end of the MTs, centrosomal or released, and is probably not involved in nucleation. There is still considerable debate about the role of ninein with respect to nucleation of MTs, as work by Delgehyr et al. has shown that ninein acts as an accessory molecule in promoting MT nucleation by docking the $\gamma$-TuRC complex at the centrosome (Delgehyr, Sillibourne, \& Bornens, 2005). 
Much like MTs that show both centrosomal as well as non centrosomal localization in neurons, ninein is also found to be localized in the centrosome as well as dispersed within the cellular matrix associated with the minus-end of these non centrosomal MTs (Baird et al, 2004; Ohama \& Hayashi, 2009). In the developing neocortex, ninein has been shown to be present in the progenitor rich ventricular zone as well as in differentiated neurons within the cortical plate. Ninein is localized to the centrosome in progenitors and is found bound to non-centrosomal microtubules of dendrites in differentiated neurons (Ohama \& Hayashi, 2009; Wang et al., 2009) . So far all the functional studies with respect to the role of ninein in the developing neocortex have pertained to the centrosomal ninein (Shinohara et al., 2013; Wang et al., 2009). The functional role of non-centrosomal ninein found in mature neurons is yet to be revealed and forms an essential part of this study.

\subsection{Transcriptional control over establishment of neocortical}

\section{projections}

As pointed out earlier for the correct establishment of neuronal circuitry as well as to propel neurons to extend axons towards the right target, a repertoire of molecules in the form of ligands and their cognate receptors, down-stream signaling molecules, cytoskeleton binding and modifying proteins are involved. Needless to say, these different molecules need to be tightly regulated so that the right molecules are expressed at the right time in both the neurons as well as in the environment. Transcription factors act as molecular switches that control the expression of these molecules both spatially and temporally. For example, Satb2 is expressed in the callosal neurons of the cortex and is required for the formation of the CC (Alcamo et al., 2008; Britanova et al., 2008). Fezf2 and Ctip2 transcription factors are expressed in deep layer neurons and are indispensible for the establishment and targeting of the CST (Arlotta et al., 2005; B. Chen et al., 2008; B. Chen, Schaevitz, \& McConnell, 2005). Similarly, other transcription factors such as Sox5, Tbr1 and Otx1 have also been shown to influence cortical projection 
neuron identity (Bedogni et al., 2010; Han et al., 2011; Hevner et al., 2002; Kwan et al., 2008; Lai et al., 2008; McKenna et al., 2011; Weimann et al., 1999). In this study we have investigated the role of three such transcription factors Sip1, Satb2 and Ctip2 that control and coordinate the establishment of the cortical axonal trajectories.

\subsection{Role of $S a t b 2$ and Ctip2 in controlling axonal trajectory choice}

Two genes, Satb2 and Ctip2 have been shown in recent years to orchestrate important and mutually exclusive genetic programs that establish corticocortical versus corticofugal projections respectively (Alcamo et al., 2008; Arlotta et al., 2005; Britanova et al., 2008). While Satb2, a matrix attachment region (MAR) interacting protein has been shown to be indispensable for the formation of the CC, Ctip2 on the other hand is required for the fasciculation and pathfinding of sub-cortically projecting neurons (Alcamo et al., 2008; Arlotta et al., 2005; Britanova et al., 2008; Szemes et al., 2006). Interestingly, Satb2 regulates the expression of Ctip2 as it binds to the upstream region of the Ctip2 locus and brings about transcriptional repression of the gene. Thus, the deletion of Satb2 in the cortex leads to an ectopic upregulation of Ctip2 in upper layer neurons, with a corresponding misrouting of callosally projecting neurons to sub-cortical targets (Alcamo et al., 2008; Britanova et al., 2008). Thus, in normal development, Satb2 overrides the Ctip2 driven molecular pathway in order to establish inter-hemispheric projections versus corticofugal projections.

\subsection{Transcriptional role of Sip1}

Sip1 (Smad interacting protein 1) also called ZFHX1b or ZEB2 is a 140kDa nuclear protein that was identified using the yeast two -hybrid system to bind to the SMAD proteins (Postigo \& Dean, 2000; Verschueren, 1999). SMADs are intracellular proteins that help in the signal transduction of 
extracellular signals to the nucleus as a result of $\mathrm{Tgf} ß$ - serine/threonine kinase receptor complex activation (Heldin et al., 1997; Kretzschmar \& Massague, 1998; Whitman, 1998). Sip1 shares a high degree of resemblance with the zinc finger homeodomain protein - $\partial \mathrm{EF} 1$ (delta-crystallin enhancer binding factor 1). Similar to $\partial \mathrm{EF} 1$, Sip1 also has conserved $\mathrm{N}$ and $\mathrm{C}$ terminal zinc finger motifs called NZF and CZF respectively that flank the homeodomain region (HD). This two-handed zinc finger domain confers DNA binding ability to Sip1. Sip1 has been shown to transcriptionaly regulate gene expression by binding to a spaced bipartite $\mathrm{CACCT}(\mathrm{G})$ sequence on the promoters of target genes through its zinc finger domains (Remacle et al., 1999). Sip1 has been shown to be an important regulator of the TGFß as well as BMP pathway. It has been shown to regulate gene expression by repressing transcription of many target genes in the TGFß and BMP pathway.

Sip1 requires the presence of activated R-SMADs i.e phosphorylated RSMADs for binding to the SMAD protein (Postigo, 2003; Verschueren, 1999). Sip1 has also been shown to bind to the corepressor CtBP (C- terminalbinding protein) to bring about repression of various genes involved in the TGFß and BMP pathway (Postigo et al., 2003). Interaction of Sip1 with the NuRD complex has also been shown to be an important interaction in influencing target gene repression (Verstappen et al., 2008). Although the role of Sip1 has been largely characterized as a transcriptional repressor (Comijn et al., 2001; Grooteclaes \& Frisch, 2000; Postigo, 2003), in certain cell contexts and with respect to certain genes, Sip1 has been shown to augment transcription in the presence and in the absence of SMADs (Yoshimoto et al., 2005), thus conferring a more global regulatory role to Sip1.

\subsection{Role of Sip1 in CNS development}

Sip1 has been shown to be involved in neurogenesis in Xenopus laevis (Eisaki et al., 2000; van Grunsven et al., 2000). In the mouse, at early embryonic stages, around E8.5, Sip1 mRNA expression can be detected in the developing 
neural epithelium and in the neural crest cells. Homozygous Sip1 mutants show a failure in neural tube closure, severely retarded growth, do not undergo embryonic turning and are not viable beyond E9.5 (Van de Putte et al., 2003).

Previous work from our lab has shown that Sip1 expression can be detected in the developing cortical plate at E12.5 in postmitotic cells. As the population of postmitotic cells grows within the cortex, the expression domain of Sip1 correspondingly expands. Expression of Sip1 mRNA can also be detected in the developing basal ganglia and in the thalamus. (Miquelajauregui et al., 2007; Seuntjens et al., 2009). Interestingly the human isoform of SIP1 shows a comparable expression patter in the human CNS. SIP1 mRNA is present in the cerebral cortex, cerebellum, corpus callosum as well as the thalamus (Cacheux et al., 2001).

In mice, Sip1 has been shown to play a very important role in hippocampal development by regulating Wnt signaling, wherein the absence of Sip1 the hippocampus is extremely reduced in size due to both cell death as well as decreased proliferation (Miquelajauregui et al., 2007). Further, seminal work from our lab has also shown that Sip1 plays a very important role in cortical feed back signaling, wherein cortical post-mitotic neurons send signals back to progenitors and alter progenitor cell fate. In the absence of Sip1 from the neocortex, there is a temporal shift in corticogenesis wherein during neurogenesis, upper layer neurons are generated earlier at the cost of deep layer neurons and at later developmental stages, glial cells are precociously generated at the cost of neurons. In the spinal cord, Sip1 plays a central role in thermal pain sensation where it controls the transduction properties of the thermal nociceptive neurons by coordinating changes in the DRG-neuron voltage gated ion channels (Jeub et al., 2011). Sip1 has also been shown to play an important role in myelination in the CNS (Weng et al., 2012). Sip1 is also highly expressed in the neural retina and in the lens placode, where in the absence of Sip1 severe deficiencies in ocular lens development could be 
noticed (Grabitz \& Duncan, 2012; Yoshimoto et al., 2005). Recently, two independent groups demonstrated the role of Sip1 in interneuron differentiation and migration (McKinsey et al., 2013; van den Berghe et al., 2013). As seen from the above examples, the role of Sip1 as a modulator of different developmental paradigms in the CNS has been well studied, but the role of Sip1 in establishing cortical projections has not been investigated. Studying the role of Sip1 in axon guidance and establishing correct cortical circuitry forms a principal aim of the study presented in this thesis.

\subsection{SIP1 and Mowat-Wilson syndrome}

Haploinsufficiency of SIP1 due to nonsense or frame shift mutations in one of the alleles of SIP1 gene has been found to cause the Mowat-Wilson syndrome (MWS). Patients suffering from this syndrome show severe mental retardation, motor deficits, epilepsy and craniofacial abnormalities etc. (Cacheux et al., 2001; Wakamatsu et al., 2001; K. Yamada et al., 2001). These effects are caused by a number of developmental disorders in the patients which frequently include microcephaly and agenesis of the corpus callosum (ACC) (Mowat, Wilson, 2003; Garavelli \& Mainardi, 2007; Moal et al., 2007; Wilson et al., 2003). Thus Sip1 is a very important molecule to be studied from a developmental as well as a clinical perspective. Since most MWS patients show ACC, studying the role of Sip1 in CC formation deserves special attention.

\subsection{Scope of the study: Transcriptional role of Sip1, Satb2 and Ctip2 in axon guidance and corpus callosum formation}

In this study we have, in a broader sense, studied different molecular programs involved in the formation of axonal tracts in the forebrain, paying special attention to the corpus callosum. More specifically, we have studied the transcriptional role of Sip1, Satb2 and Ctip2 that modulate the expression of multiple axon guidance cues, guidepost structures and cytoskeletal 
molecules. We have shown that mice lacking Sip1 in the cortex show many anomalies in cortical projections. We have further dissected out the cell autonomous and non-cell autonomous role of Sip1 in the formation of the CC. We have also studied at the role of transcription factors Satb2 and Ctip2 in controlling the trajectory choice made by deep layer neurons between taking a medial route forming the CC or a lateral trajectory forming the corticofugal tract. Here, we show that Ctip2 and Satb2 transcriptionally regulate a set of axon guidance molecules that help in deciding the axonal trajectory taken. 


\section{MATERIALS AND METHODS}

\section{$\underline{2.1 \text { Mouse mutants used }}$}

All mouse experiments were carried out in compliance with the German law and were approved by the Bezirksregierung Braunschweig, the Landesamt für Gesundheit und Soziales Berlin and the University of Queensland animal ethics committee.

\subsubsection{Sip1 conditional knockout}

The Sip1 floxed mice generated by Higashi et al., was used for the work presented in this thesis (Higashi et al., 2002). Lox-p sites were inserted flanking the exon 7 of the Sip1 allele, which renders the protein inactive after Cre mediate excision. In order to generate Sip1 conditional mutant, where Sip1 has been deleted from postmitotic cells within the cortex, Sip 1 flfl mice were mated with Nex Cre mice (Goebbels et al., 2006). In these mice, Cre recombinase is expressed under the Nex promoter, which ensures expression only within the postmitotic population of the neocortex. Sip $1^{f / w t} \mathrm{Nex}{ }^{\mathrm{Cre}}$ animals obtained from this cross were used as parents to generate the Sip1 conditional knockouts designated as Sip $1^{f l / f l} N e x^{C r e}$ mice. Since Sip $1^{f / w t} N e x^{w t / w t}$, Sip $1^{f l / f} N e x^{w t / w t l}$, Sip $1^{f / w t}$ Nex Cre, littermates did not show any deviation from the wild type phenotype, they were interchangeably used as controls for the various experiments performed.

\subsubsection{Satb2 null mice}

The Satb2 null mice described in (Britanova et al., 2006) have been used in this work. Here, the Satb2 null allele was generated through homologous recombination by elimination of the second exon in the protein-coding region and replacement with a Cre recombinase-coding sequence. Satb2 ${ }^{+/-}$mice were treated as wild type. 


\subsubsection{Ctip2 null mice}

Ctip2 null mice used in the experiments here were produced by conventional gene targeting described in (Wakabayashi et al., 2003). The animals were maintained either as Ctip2+/- heterozygous parents or Satb2+-; Ctip2+/- double heterozygous parents.

\subsubsection{Netrin1 and Unc5C mutants}

The Netrin 1 hypomorphs and the Unc5C null mutants used were generated as described in (Ackerman et al., 1997; Serafini et al., 1996).

\section{$\underline{2.2 \text { Genotyping }}$}

Tail tip of 3 weeks old mice was used for genotyping. Tail tissue was digested in $0.3 \mathrm{ml}$ Lysis buffer $(100 \mathrm{mM}$ Tris- $\mathrm{HCl}$ pH8.5, $5 \mathrm{mM}$ EDTA, $200 \mathrm{mM} \mathrm{NaCl}$, $0.2 \% \mathrm{SDS}, 100 \mu \mathrm{g} / \mathrm{ml}$ Proteinase $\mathrm{K}$ ) at $55^{\circ} \mathrm{C}$ for $2 \mathrm{hrs}$ to overnight. The samples were centrifuged at $9000 \mathrm{rpm}$ for $10 \mathrm{mins}$ to remove un-lysed tissue and residual hair. The DNA in the supernatant was precipitated by adding an equal volume of isopropanol, followed by gently mixing and centrifugation at $13000 \mathrm{rpm}$ for $15 \mathrm{mins}$. The DNA precipitate was washed twice in $80 \%$ ethanol, air dried and resuspended in 50 to $100 \mu \mathrm{l}$ sterile distilled water.

All PCR reactions were done in a final volume of $20 \mu \mathrm{l}$ in a mixture prepared according to the following protocol-

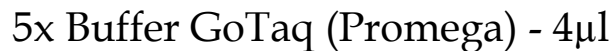

10mM dNTPs (Invitrogen) - 0.4 $\mu \mathrm{l}(20 \mathrm{pmol} / \mathrm{ml})$

Forward/Reverse primers (IBA) $-1 \mu \mathrm{l}(10 \mathrm{pmol} / \mathrm{ml})$ each

GoTaq polymerase (Promega) - 0.2 $\mu \mathrm{l}$ (0.5 units)

Template DNA - $1 \mu 1$

$\mathrm{ddH}_{2} \mathrm{O}-12.4 \mu \mathrm{l}$ 
The following primer sequences were used to detect the amplified product:

\subsubsection{Sip1-floxed and wild type alleles:}

Forward- 5' TGGACAGGAACTTGCATATGCT 3'

Reverse- 5' GTGGACTCTACATTCTAGATGC 3'

Amplification program-

$94^{\circ} \mathrm{C}-10^{\prime \prime}$

$59^{\circ} \mathrm{C}-20^{\prime \prime}$

$72^{\circ} \mathrm{C}-40^{\prime \prime}$

for 31 cycles;

Wild type allele yields a product of $\sim 450 \mathrm{bp}$ and the floxed Sip 1 allele yields a product of $\sim 600 \mathrm{bp}$.

\subsubsection{Cre allele (for Nex-Cre)}

Forward- 5' TCGATGCAACGAGTGATGAG 3'

Reverse- 5' TTCGGCTATACGTAACAGGG 3'

Amplification program-

$94^{\circ} \mathrm{C}-10^{\prime \prime}$

$55^{\circ} \mathrm{C}-30^{\prime \prime}$

$72^{\circ} \mathrm{C}-40^{\prime \prime}$

30 cycles;

Presence of at least one Cre allele yields a product of $\sim 500 \mathrm{bp}$.

\subsubsection{Satb2 null allele}

To detect $w t$ and Satb2 KO alleles

5' - CAAGAGAGCCATCCAACTGC- 3'

a reverse primer that recognizes Cre 5' - CCAGACCGCGCGCCTGAAGA- 3'

and 5'- AACCATCAGGCTCAACC3'

Amplification program-

$94^{\circ} \mathrm{C}-10^{\prime \prime}$

$55^{\circ} \mathrm{C}-30^{\prime \prime}$

$72^{\circ} \mathrm{C}-40^{\prime \prime}$

30 cycles; 
In $w t$ mice, the PCR generated a fragment of $\sim 400 \mathrm{bp}$ where as mutant alleles generated a fragment of $\sim 200 \mathrm{bp}$.

All PCR products were analyzed by electrophoresis on a 1.2-1.4\% agarose gel at $90-120 \mathrm{~V}$. The products were separated in TAE buffer ((40mMTris- acetate, 1mMEDTA, $\mathrm{pH} 8$ ) containing 1: $1 \times 10^{5}$ Serva green (Serva Electrophoresis), and were visualized under UV illumination in a INTAS gel documentation system. The size of the PCR products was determined by comparing with standard DNA ladders (Bioline) loaded at 200ng/ $\mu 1$.

\section{$\underline{2.3 \text { Tissue processing }}$}

\subsubsection{Embryonic and early postnatal tissue}

The day of the vaginal plug was considered as embryonic day 0.5 and the required embryonic stage for experiments was accordingly calculated. Pregnant females at the appropriate stage were sacrificed by administration of an overdose of Avertin, followed by cervical dislocation. The embryos were dissected out of the uterus into ice-cold 1X PBS and brains were isolated. The brains were fixed in $4 \%$ PFA in $1 \mathrm{X}$ PBS at $4^{\circ} \mathrm{C}$ and the duration was decided based on the embryonic age.

E12.5 to E13.5: 4 to 6 hours

E16.5 to E18.5: 8 to 10 hours

P0 to P4: 10 to 14 hours

P4 and above: perfusion followed by overnight (o/n) incubation

The fixed tissue was then washed twice or thrice with 1X PBS. The brains were further processed based on the type of histological sections required.

\subsubsection{Perfusion}

Mice older than P5 were fixed using perfusion with 4\% PFA before immersing in the same solution overnight in order to ensure proper fixation of the tissue. 
The mice were anesthetized by intraperitoneal administration of Avertin (100$300 \mu 1$ per animal, depending on the age). Subsequently, they were dissected open to expose the chest-cavity, rib cage was cut, followed by the diaphragm. A fine needle connected to a syringe with cooled PBS was inserted into the left ventricle and held in position. A small nick was made in the right auricle to expel the blood. This ensured circulation of PBS through the entire circulatory system, facilitated initially by the beating of the heart. As a sign of successful perfusion, visceral organs such as the liver can be seen to turn pale in color. The PBS was then replaced with $4 \%$ PFA and the perfusion was continued for a few minutes until certain prominent signs of penetration of PFA into tissues was detected. The signs would include, movement of the head (in case of young pups), movement of the tail (in juvenile and adult mice) etc. After this, the brain was dissected out (should appear pale and devoid of any blood in the vessels), and immersed in 4\% PFA overnight.

\subsubsection{Cryo-sections}

The brains were taken through a series of sucrose gradients starting from $7 \%$ Sucrose in 1X PBS, followed by 15\% sucrose and finally in 30\% Sucrose. The brains were allowed to equilibrate in each solution till the tissue sunk to the bottom of the well. The brains were then washed one time in OCT (TissueTek) and placed in an embedding mould immersed in fresh OCT. The mould was then placed on a slab of dry ice to freeze the sample. The sample was then stored at $-20^{\circ} \mathrm{C}$ or $-80^{\circ} \mathrm{C}$. Coronal sections with a thickness of $12 \mu \mathrm{m}$ to $20 \mu \mathrm{m}$ were cut using a Cryotome (Leica). Sections were collected on Superfrost plus slides (Menzel- Gläser) and stored at $-20^{\circ} \mathrm{C}$ or $-80^{\circ} \mathrm{C}$ till use.

\subsubsection{Paraffin sections}

The fixed brains were incubated overnight in $0.9 \% \mathrm{NaCl}$, followed by progressive dehydration through a series of increasing strength of ethanol (50\% to $100 \%$ ), followed by isopropanol. They were then incubated in toluol 
for atleast $6 \mathrm{hrs}$, followed by Paraplast wax (maintained at $60^{\circ} \mathrm{C}$ ) (twice, overnight). Finally they were embedded in wax within plastic chambers and sectioned into $10 \mu \mathrm{m}$ thick frontal sections using a Microtome (Leica). The sections were mounted on silane- coated slides (Marienfeld Histobond).

\subsubsection{Vibratome sections}

The fixed brains were embedded in melted 4\% Agarose in 1X PBS and allowed to solidify. The block was then sectioned using a Vibratome (Leica) with a thickness varying between 60 to $100 \mu \mathrm{m}$. The floating sections were collected with a brush in 1X PBS with $0.1 \%$ Sodium Azide used as an antifungal agent.

\section{$\underline{2.4 \text { Histological staining }}$}

\subsubsection{Nissl Staining}

Paraffin section of the brains were used for Nissl staining. The slides were firstly de-paraffinized by immersing them in Xylol for 10 minutes with 1 change. The sections were then re-hydrated by taking them through a gradient of decreasing concentration of ethanol from $100 \%$ ethanol to $30 \%$ ethanol for 2 minutes each ending in two washes in 1X PBS. The slides were then washed in double distilled $\mathrm{H}_{2} \mathrm{O}$ and then incubated in $50 \%$ potassium disulfite for $15 \mathrm{mins}$. They were washed again, and stained in Cresyl violet $(1.5 \%$ in acetate buffer) for approx. 20mins. The sections were then washed twice in acetate buffer $\left(10 \mathrm{mM}\right.$ sodium acetate, $10 \mathrm{mM}$ acetic acid in $\left.\mathrm{H}_{2} \mathrm{O}\right)$ for 2 mins each to remove excess stain. This was followed by a very quick dip in Differentiation buffer $(0.14 \%$ glacial acetic acid $)$ until the desired color/intensity was obtained. The slides were then dehydrated again in $70 \%$ ethanol, followed by two-2 minute incubations in $100 \%$ ethanol. The slides were then immersed in Xylol for 10 minutes with 1 change. Finally, the 
sections were mounted using a Xylol- based mounting medium (Eukitt) and dried before imaging.

\subsubsection{Immunohistochemistry using fluorescent primary and secondary} $\underline{\text { antibodies }}$

The pre-treatment protocol for immunohistochemistry was slightly modified depending on the type of sections used. For paraffin sections, the slides were first re-hydrated in decreasing concentration of ethanol in $\mathrm{H}_{2} \mathrm{O}$ from $100 \%$ to $30 \%$ for 2 minutes each followed by two 5 minute washes in $1 \times$ PBS. The sections were then subjected to antigen retrieval by boiling the slides for 3 mins in a preheated $0.96 \%$ antigen unmasking solution (Vector Labs), followed by cooling to $50^{\circ} \mathrm{C}$, heating again for 3 mins in the unmasking solution, and finally cooling to room temperature. The slides were then rinsed in PBS for 10mins. In case of cryosections, the slides were brought to room temperature and washed in 1X PBS for 10 minutes to remove the dried OCT surrounding the sections. In case of vibratome sections, no pre-treatment was required. The sections were then blocked to prevent background staining in 2\% BSA/0.3\% TritonX-100 in 1X PBS for 1 hour at room temperature (RT). Primary antibody cocktail at the appropriate dilution was made in the same blocking solution and sections were incubated in it overnight at $4^{\circ} \mathrm{C}$. Three rounds of $15 \mathrm{~min}$ washes in PBS at RT followed this. The sections were then incubated in secondary antibody cocktail made in blocking solution for 1 hour at RT. The secondary antibodies used were AlexaFluor- tagged secondary antibodies (1:500, Molecular Probes) or DyLight conjugated secondary antibodies (1:500, Jackson Labs). In some cases nuclear stain DRAQ5 was used along with the secondary antibodies (1:1000, eBioscience). The slides were finally mounted in a fluorescent mounting medium (DAKO Cytomation). 


\subsubsection{Immunohistochemistry using alkaline phosphatase based} chromogenic reaction

The sections were pre-processed as mentioned above. In this case all types of sections including cryosection and vibratome sections were subjected to antigen retrieval or incubation at $65^{\circ} \mathrm{C}$ for at least 1 hour before blocking. This was done to inactivate endogenous alkaline phosphatase (AP). The blocking solution was made in TBS and all washes were also performed using 1X TBS. After overnight incubation at $4^{\circ} \mathrm{C}$ with primary antibody the sections were washed 3 times for 15 minutes with 1X TBS. Secondary antibody conjugated to AP (Promega) was used at 1:500 dilution in blocking solution for 1 hour incubation at RT followed by at least two 10 minute washes with 1X TBS and one wash with NTMT, pH 9.5. The AP substrate NBT/BCIP (Roche) in NTT (1:50) was added to the sections and incubated till the purple precipitate developed to the required intensity. The enzymatic reaction was stopped with two washes in 1X PBS and the sections were mounted using a fluorescent mounting medium (DAKO Cytomation).

\section{$\underline{1 X \text { TBS (Tris Buffer Saline) }}$}

50 mM Tris-Cl, $\mathrm{pH} 7.5$

$150 \mathrm{mM} \mathrm{NaCl}$

To prepare, dissolve $6.05 \mathrm{~g}$ Tris and $8.76 \mathrm{~g} \mathrm{NaCl}$ in $800 \mathrm{~mL}$ of $\mathrm{H}_{2} \mathrm{O}$. Adjust $\mathrm{pH}$ to 7.5 with $1 \mathrm{M} \mathrm{HCl}$ and make volume up to $1 \mathrm{~L}$ with $\mathrm{H} 2 \mathrm{O}$.

NTMT (Alkaline Phosphatase buffer)- 200ml

$100 \mathrm{mM} \mathrm{NaCl}(4 \mathrm{ml}$ of $5 \mathrm{M} \mathrm{NaCl})$

$100 \mathrm{mM}$ Tris-Cl, pH 9.5 (20ml of $1 \mathrm{M}$ Tris-Cl, $\mathrm{pH} 9.5)$

$50 \mathrm{mM} \mathrm{MgCl} 2\left(10 \mathrm{ml}\right.$ of $\left.1 \mathrm{M} \mathrm{MgCl}_{2}\right)$

$1 \%$ Tween $20(2 \mathrm{ml})$

$\mathrm{H}_{2} \mathrm{O}$ to $200 \mathrm{ml}$

NTT (Alkaline phosphate buffer without Magnesium salt) - $200 \mathrm{ml}$ $100 \mathrm{mM} \mathrm{NaCl}(4 \mathrm{ml}$ of $5 \mathrm{M} \mathrm{NaCl})$ 
100mM Tris-Cl, pH 9.5 (20ml of 1 M Tris-Cl, $\mathrm{pH} 9.5)$

$1 \%$ Tween $20(2 \mathrm{ml})$

$\mathrm{H}_{2} \mathrm{O}$ to $200 \mathrm{ml}$

\subsubsection{Immunocytochemistry}

Coverslips covered with cultured cells were first fixed in $4 \%$ PFA with $4 \%$ Sucrose in 1X PBS for $20 \mathrm{~min}$ at RT. They were then washed twice in PBS for $10 \mathrm{~min}$ per wash, and left overnight in the same solution at $4^{\circ} \mathrm{C}$. The cells were firstly permeabilised in $0.1 \%$ Tween 20 in PBS for $15 \mathrm{~min}$ at RT, followed by blocking of non- specific binding sites with the blocking solution (2\% BSA in $0.1 \%$ Tween20 in PBS) for $1 \mathrm{hr}$ at RT in a humid chamber. The coverslips were then treated with the primary antibodies prepared in blocking solution overnight at $4^{\circ} \mathrm{C}$. They were then washed three times in PBS for 15 min per wash, and incubated for in 1:500 diluted secondary antibody solution for $1 \mathrm{hr}$ at RT. Finally, excess secondary antibodies were washed off twice in PBS (10min each) and the coverslips were mounted in fluorescent mounting medium (DAKO Cytomation). 
2.4.5 Antibodies used

\begin{tabular}{|l|l|l|l|}
\hline \multicolumn{1}{|c|}{ Name } & \multicolumn{1}{c|}{ Species } & \multicolumn{1}{c|}{ Company } & \multicolumn{1}{c|}{ Dilution } \\
\hline Ac-tubulin & Rabbit & Sigma & $1: 3000$ \\
\hline Calretinin (CR) & Rabbit & Swant & $1: 1000$ \\
\hline Cre & Mouse & Sigma & $1: 500$ \\
\hline Ctip2 & Rat & Abcam & $1: 250$ \\
\hline DCC & Rabbit & H.Cooper & $1: 100$ \\
\hline dsRed & Rabbit & Clonetech & $1: 250$ \\
\hline GFAP & Mouse & Sigma & $1: 500$ \\
\hline GFP (1) & Goat & Rockland & $1: 500$ \\
\hline GFP (2) & Chicken & Abcam & $1: 1000$ \\
\hline L1 & Rat & Millipore & $1: 200$ \\
\hline Myc & Goat & Abcam & $1: 2000($ W.B) \\
\hline Ninein & Rabbit & K. Hayashi & $1: 500$ \\
\hline RFP (1) & Rabbit & Abcam & $1: 250$ \\
\hline Satb2 & Rabbit & Self Generated & $1: 1000$ \\
\hline Sip1 & Rabbit & Self generated & $1: 500$ \\
\hline Tau-1 & Mouse & Millipore & $1: 400$ \\
\hline Unc5C & Rabbit & S. Ackerman & $1: 300$ \\
\hline
\end{tabular}

\subsubsection{In-situ hybridization}

\section{Cloning and transformation}

$800 \mathrm{bp}$ to $1000 \mathrm{bp}$ sequence within the coding region of the gene of interest was chosen and forward and reverse primers were designed accordingly. Using E16.5 mouse cDNA as template the selected region was amplified through PCR. GoTaq polymerase was used for the PCR amplification as it provides a 3 ' deoxyadenosine ' $A$ ' over hang in the amplicon. The amplicon was ligated into pGEMT plasmid vector (Promega) through TA ligation. The ligated 
plasmid was transformed into $\mathrm{DH} 5 \alpha, \mathrm{XL}-10 \mathrm{G}$ or $\mathrm{XL}-10$ blue competent bacteria. For this the plasmid mix was incubated with $100 \mu \mathrm{l}$ of competent cells on ice for 10 minutes followed by a heat shock at $42^{\circ} \mathrm{C}$ for 60 to 90 seconds and immediate cooling on ice for 5 minutes. The cells were then grown for 1 hour at $37^{\circ} \mathrm{C}$ in I $\mathrm{ml} \mathrm{LB}$ media without antibiotic in a shaker. The cells were then pelleted down, resuspended in $100 \mu \mathrm{LB}$ and plated on LB agar plates with ampicillin. For blue white selection of the colonies the LB agar plated were also coated with X-gal/IPTG before plating out the cells. The plates were incubated at $37^{\circ} \mathrm{C}$ over night. Colonies were picked the next day and grown in $5 \mathrm{ml} \mathrm{LB}$ media with ampicillin followed by plasmid extraction using Qiagen's mini-prep kit and columns. The presence of the DNA sequence of interest and the orientation was verified using restriction enzyme digestion and appropriate clones were selected and glycerol stocks of the culture was made and stored at $-80^{\circ} \mathrm{C}$. All in situ probes used in this study were obtained from Kuo Yan, Institute for Cell and Neurobiology, CharitéBerlin.

\section{Plasmid linearization}

The probe sequences (cloned into plasmids) were flanked by two different types of promoters (usually T3, T7 or Sp6) oriented in such a way that they could be used to synthesize either the antisense or the sense RNA. Therefore, depending on the requirement, the purified plasmids were linearised using a specific restriction enzyme (New England Biolabs) that would cleave the cDNA sequence at the end opposite to the promoter that will be used subsequently for probe synthesis. $1-5 \mu \mathrm{g}$ of plasmid DNA was digested per reaction. 10units of restriction enzyme was used for linearising $1 \mu \mathrm{g}$ plasmid DNA. Reactions were carried out at $37^{\circ} \mathrm{C}$ for 5 hrs until the linearization was completed, which in turn was tested by gel electrophoresis. The linearized DNA was extracted from the gel by cutting out the portion of the gel with the linearized DNA band and processed using Qiagen- DNA gel extraction kit as per manufacturers instructions. 


\section{Synthesizing ribo-probe}

Linearized plasmid DNA

$1 \mu l$

DIG RNA labeling mix (Roche)

$2 \mu 1$

10x transcription buffer (Roche)

$2 \mu 1$

RNase inhibitor (Roche)

$1 \mu \mathrm{l}(20 \mathrm{U})$

RNA polymerase (T7, T3, Sp6) (Roche)

$1 \mu 1$

DEPC treated $\mathrm{H}_{2} \mathrm{O}$

$13 \mu 1$

Incubate for 2 hours at $37^{\circ} \mathrm{C}$ and purify using RNA cleanup kit (QIAGEN).

\section{In-situ hybridization: Tissue processing and probe application (Day 1)}

The embryos at the required stage were sacrificed and the brains were dissected in ice cold 1X PBS treated with DEPC to make it RNase free. The brains were then fixed overnight in 4\% PFA-7\% Sucrose in 1X DEPC treated PBS followed by incubation in 15\% Sucrose and 30\% Sucrose and finally cryoembedding in OCT. 14 to $16 \mu \mathrm{m}$ sections were cut using a cryotome and the sections were collected and dried on Superfrost slides for about 30 minutes. The sections were then post fixed in 4\% PFA for 15 minutes followed by two washes with PBS. All solutions used on day 1 were treated with DEPC to make them RNase free. The sections were then treated with ProtienaseK (at $20 \mu \mathrm{g} / \mathrm{ml}$ ) for 3 minutes at RT to sufficiently permeablize the tissue. Following this step, the slides were immediately immersed in $0.2 \%$ Glycine in 1 X PBS for 5 minutes followed by two washes with 1x PBS. The sections were then treated with pre-hybridisation mix (50\% formamide, 5x SSC pH7.0, 2.5M EDTA, 0.1\% Tween-20, 0.15\% CHAPS, 0.1mg/ml Heparin, 100 $\mu \mathrm{g} / \mathrm{ml}$ yeast tRNA, $50 \mu \mathrm{g} / \mathrm{ml}$ salmon sperm DNA, 1x Denhardt's solution) for two hours at $65^{\circ} \mathrm{C}$ followed by incubation with RNA probe diluted in the hybridization mix, and incubated overnight at $65^{\circ} \mathrm{C}$.

\section{In situ hybridization: Blocking and antibody application (Day 2)}

On the second day the tissue was treated with RNase A in RNase buffer for 30 minutes at $37^{\circ} \mathrm{C}$ followed by 3 washes with $2 \times$ SSC, pH 4.5 in $50 \%$ formamide at $65^{\circ} \mathrm{C}$ for 30 minutes each. The slides were then immersed in KTBT solution 
for 5 minutes and then the tissue was blocked using 20\% sheep serum. Sheep anti-DIG antibody (Roche, 1:1000) was applied overnight at $4^{\circ} \mathrm{C}$.

\section{In situ hybridization: washes and developing (Day 3)}

On the third day, the tissue was washed in 6 times in KTBT and 4 times in NTMT. The reaction was developed by adding substrate NBT/BCIP (Roche, chromogenic) at 1:50, diluted in NTT solution. Upon reaching appropriate signal intensity, the reaction was stopped with atleast two washes in PBS. The sections were dehydrated with increasing concentration of ethanol and mounted using Eukitt mounting media.

\section{Solutions used}

\section{X PBS- DEPC}

$1 \mathrm{ml}$ of DEPC is added to 11 XPBS, Shake $\mathrm{o} / \mathrm{n}$ at $37^{\circ} \mathrm{C}$, autoclave

\section{$\underline{1 X \text { PBST }}$}

\section{1XPBS}

$1 \mathrm{ml}$ Triton X-100 (0.1\%)

\section{$\underline{\text { Proteinase K buffer }}$}

$30 \mathrm{mM}$ Tris $\mathrm{pH} 8.0(30 \mathrm{ml}$ of $1 \mathrm{M}$ Tris $\mathrm{pH} 8.0)$

10mM EDTA pH 8 (50ml of 0.2M EDTA)

$\mathrm{H}_{2} \mathrm{O}$ to $1000 \mathrm{ml}$

\section{$\underline{\operatorname{KTBT}(11)}$}

$150 \mathrm{mM} \mathrm{NaCl}(30 \mathrm{ml}$ of $5 \mathrm{M} \mathrm{NaCl})$

$50 \mathrm{mM}$ Tris-Cl, pH 7.5 (50ml of 1 M Tris-Cl, $\mathrm{pH} 7.5)$

$10 \mathrm{mM} \mathrm{KCl}(10 \mathrm{ml}$ of $1 \mathrm{M} \mathrm{KCl})$

1\% TritonX-1000 (10ml)

$\mathrm{H}_{2} \mathrm{O}$ to $1000 \mathrm{ml}$

NT(M)T (Alkaline Phosphatase buffer)- 500ml

$100 \mathrm{mM} \mathrm{NaCl}(10 \mathrm{ml}$ of $5 \mathrm{M} \mathrm{NaCl})$ 
100mM Tris-Cl, pH 9.5 (50ml of 1 M Tris-Cl, pH 9.5)

$50 \mathrm{mM} \mathrm{MgCl} 2\left(25 \mathrm{ml}\right.$ of $\left.1 \mathrm{M} \mathrm{MgCl}_{2}\right)$

$0.05 \%$ Tween $20(250 \mu \mathrm{l})$

$\mathrm{H}_{2} \mathrm{O}$ to $500 \mathrm{ml}$

$\underline{\text { RNase Buffer }}$

$15 \mathrm{ml} 5 \mathrm{M} \mathrm{NaCl}$

$1.5 \mathrm{ml}$ 1M Tris $\mathrm{pH} 7.5$

$\mathrm{H}_{2} \mathrm{O}$ upto $150 \mathrm{ml}$

\subsubsection{Immunofluorescence - Fluorescence in situ hybridization (IF-} $\underline{\text { FISH) }}$

The IF-FISH protocol was similar to the in situ hybridization protocol with certain specific modifications. The ProtienaseK step on day 1 was omitted for the IF-FISH protocol and was instead substituted with a 25-minute incubation in 2 X SSC pH7.0. On day 2, for the IF-FISH, antibodies against Satb2 and Ctip2 were included together with anti DIG antibody. On the third day, the reaction was developed by adding substrate Fast Red (Abcam). Upon reaching appropriate signal intensity, for the IF-FISH, the sections were washed in PBS and stained with appropriate secondary antibodies.

\section{$\underline{2.5 \text { DiI labeling }}$}

For anterograde cortical axon tracing, after fixing the isolated brains in $4 \%$ PFA, crystals of DiI (1,1'-dioctadecyl-3,3,3', $3^{\prime}$-tetramethylindocarbocyanine-perchlorate) (Molecular probes) were placed with the help of a tungsten electrode used to make a fine incision into the somato-sensory, motor and visual cortical areas of the brain. After DiI placement, brains were kept for 2 weeks in case of P0 brains and 3-4 weeks in case of P5 brains at $37^{\circ} \mathrm{C}$ immersed in PBS with $0.1 \%$ Sodium Azide in the dark. 
Alternatively, for retrograde tracing of cortico-thalamic or cortico-spinal tract, DiI crystals were placed in the thalamus or the cerebral peduncle. For this the brain was firstly cut coronally at the appropriate rostro-caudal level and then the dye was placed in the respective structures using the tungsten electrode. Methyl green solution was added onto the brains to increase the contrast and aid in crystal placement at the correct position.

After incubation for the stipulated time, brains were embedded in $4 \%$ Agarose (Applichem) and sectioned into $100 \mu \mathrm{m}$ thick sections using a vibratome (Leica). The sections obtained were counterstained with Hoechst (1:1000, Sigma) and mounted in PBS. Alternatively sections were processed for immunostaining with Satb2. No detergent was used for staining DiI labeled sections. Images were procured on a Leica SL/SP2 confocal microscope and counting was done manually. Statistics was done using $\mathrm{R}$ and Microsoft excel. Graphs were made on GraphPad prism 5.

\subsection{Image acquisition}

Bright field and fluorescence images were acquired using Olympus BX51 and BX60. Confocal images were acquired using Leica Confocal System-SL.

\section{$\underline{2.7 \text { In utero electroporation }}$}

This procedure was carried out as described before (Saito, 2006). Pregnant and staged female was anesthetized in a chamber using isofluran. The animal was then placed on its back on a heating pad while constantly inhaling isofluran to stay anesthetized. Analgesic was administered subcutaneously. A vertical incision was made in the abdominal area and the uterine horns were carefully retrieved from the female without damaging the blood vessels and other visceral organs. The plasmid solution together with fast green dye used for visualization was filled into a fine capillary that was pulled using HEKA- 
PIP6 capillary puller. The sharp end of the capillary was inserted into the head of the embryo so as to penetrate the skull and enter the lateral ventricles of the brain. Using a foot pedal pump, the plasmid was injected into the lateral ventricle. A square wave pulse consisting of $35 \mathrm{mV}$ current in 6 pulses was administered using an electrode and BTX electroporator. The uterus was constantly kept moist by addition of 1X PBS with penicillin streptomycin maintained at $37^{\circ} \mathrm{C}$. This was then repeated for all the embryos and the uterus was re-inserted into the body cavity of the mouse. The body wall was sutured using sterilized sutures and the skin was clipped together using wound clips (Autoclip). The female was allowed to recover and put back into her cage. The female was monitored over the next days to ensure that the animal was normal. The embryos were then harvested at the chosen embryonic or postnatal stage.

\subsection{Plasmids used}

\begin{tabular}{|c|c|c|c|}
\hline Plasmid name & Promoter & Protein expressed & Source \\
\hline pFUGW-Cre & Ubiquitin & GFP IRES Cre & $\begin{array}{ll}\text { Dr. } & \text { Hiroshi } \\
\text { Kawabe } & \end{array}$ \\
\hline pCAG-Cre & CAG & Cre & $\begin{array}{l}\text { Generated in the } \\
\text { lab }\end{array}$ \\
\hline$p C A G e G F P$ & CAG & eGFP & $\begin{array}{l}\text { Generated in the } \\
\text { lab }\end{array}$ \\
\hline$p C A G$ dsRed & CAG & dsRed & $\begin{array}{ll}\text { Dr. } & \text { Gregory } \\
\text { Wulczyn } & \end{array}$ \\
\hline$p C A G$ FMF eGFP & CAG & $\begin{array}{l}\text { eGFP in the presence of } \\
\text { Cre }\end{array}$ & Self generated \\
\hline$p C A G$ FMF Sip1-myc & CAG & $\begin{array}{l}\text { Sip1-myc in the } \\
\text { presence of Cre }\end{array}$ & $\begin{array}{l}\text { Generated in the } \\
\text { lab }\end{array}$ \\
\hline$p C A G$ FMF eGFP-Ninein & CAG & $\begin{array}{l}\text { eGFP-Ninein in the } \\
\text { presence of Cre }\end{array}$ & $\begin{array}{l}\text { Generated in the } \\
\text { lab }\end{array}$ \\
\hline$p C M V$ eGFP-Ninein & CMV & eGFP-Ninein & Dr. Michel Bornens \\
\hline$p C A G$ Ninein & CAG & Ninein & $\begin{array}{l}\text { Generated in the } \\
\text { lab }\end{array}$ \\
\hline
\end{tabular}




\begin{tabular}{|l|l|l|l|}
\hline$p C M V$ GFP-EB3 & CMV & GFP-EB3 & $\begin{array}{l}\text { Dr. } \\
\text { Stegmüller }\end{array}$ \\
\hline$p C A G$ GFP-EB3 & CAG & GFP-EB3 & $\begin{array}{l}\text { Generated in the } \\
\text { lab }\end{array}$ \\
\hline$p C \beta A-F L E x$ & B-actin & $\begin{array}{l}\text { tdTomato when Cre is } \\
\text { absent and eGFP in the } \\
\text { presence of Cre }\end{array}$ & Dr. Ulrich Müller \\
\hline$p C M V$ Ctip2 & CMV & Ctip2 & Dr. Leid \\
\hline$p C A G$ Ctip2 & CAG & Ctip2 & $\begin{array}{l}\text { Generated in the } \\
\text { lab }\end{array}$ \\
\hline$p C A G$ Venus & CAG & Venus GFP & Dr. Handjantonakis \\
\hline$p C A G$ Satb2 & CAG & Satb2 & Dr. Nenad Sestan \\
\hline$p C A G$ Ski & CAG & Ski & Dr.Suzana \\
\hline$p C A G$ Unc5C & CAG & Unc5C & Dr. Franck Polleux \\
\hline$p C A G$ DCC-myc & CAG & DCC-myc & Dr. Helen Cooper \\
\hline$p U 6$ DCCshRNA2 & U6 & DCCshRNA & Genecopoiea \\
\hline$p U 6$ DCCshRNA4 & U6 & DCCshRNA & Genecopoiea \\
\hline
\end{tabular}

All the plasmids generated in the lab were cloned by Srinivas Parthasarathy, Institute for Cell and Neurobiology, Charité, Berlin.

\section{$\underline{2.9}$ DCC knockdown assay using shRNA}

DCC shRNA clones were ordered from GeneCopoeia with the following target sequences

DCC shRNA 1: aagccggatgaaggacttt

DCC shRNA 2: agagaccatccaacgtaat

DCC shRNA 3: ttgtcgcctacaatgagtg

DCC shRNA 4: agcagcggtactattccat

The DCC shRNA plasmids were expressed in HEK-293T cells together with a plasmid coding for DCC-myc using Lipofectamine 2000 (Invitrogen). An unrelated RNA sequence was used for the scrambled shRNA. The cells were lysed after 2 days in lysis buffer (50mM Tris- $\mathrm{HCl} \mathrm{pH} 7.5,150 \mathrm{mM} \mathrm{NaCl}, 1 \mathrm{mM}$ 
EDTA, 1\% Triton X-100, 1x protease inhibitor cocktail (Sigma), pepstatin). Lysates were centrifuged for 20 minutes at $12000 \mathrm{~g}, 4^{\circ} \mathrm{C}$. Protein concentration was measured using BCA assay (Thermo scientific) and $30 \mu \mathrm{g}$ protein was loaded on a SDS-PAGE gel and transferred onto a PVDF membrane. The membrane was subsequently blocked for one hour in 3\% BSA in TBST (TBS + 0.5\% Tween-20), followed by overnight incubation in anti-Myc (1:2000) and anti-tubulin (1:50000) antibodies at $4^{\circ} \mathrm{C}$. The membranes were washed in TBST on the next day and incubated for one hour in peroxidase coupled secondary antibodies (Jackson Laboratory). Chemiluminescence (ECL western blotting detection reagent, Perkin Elmer) was used for visualizing the protein bands. The chemiluminescence was captured using the Image lab software on a ChemiDoc XRS+ detector (Bio-Rad). The efficiency of knockdown was calculated using ImageJ.

\section{$\underline{\text { 2.10 Dissociated cortical neuron culture }}$}

On the previous day, sterile coverslips were placed in a 24 well plate and coated with poly-L-lysine at $0.1 \mathrm{mg} / \mathrm{ml}$ concentration and kept at $37^{\circ} \mathrm{C}$ over night. The next day the plates were washed with water or PBS three times and the plates were left to dry within the cell culture hood. On the day of culture E16.5/E17.5 embryos were harvested and brains was isolated on ice cold 1X HBSS with Calcium and Magnesium - HBSS [++] (GIBCO). The two cerebral hemispheres were separated and the meninges were removed. The basal ganglia and the hippocampus were dissected out and only the cortex was harvested. If the brains were previously electroporated then the cortices were visualized under a fluorescent binocular and only the fluorescent tissue was dissected out. The tissue was pooled from the entire litter and collected in a $15 \mathrm{ml}$ falcon in HBSS [++]. The tissue was washed three times with $10 \mathrm{ml}$ HBSS without Calcium and Magnesium HBSS - - (GIBCO). After the last wash $3.5 \mathrm{ml}$ of HBSS [- -] was left in the falcon and $500 \mu \mathrm{l}$ of $2.5 \%$ Trypsin (GIBCO) was added to it and incubated at $37^{\circ} \mathrm{C}$ for 30 minutes. $2 \mathrm{ml}$ of heat inactivated 
horse serum was added to the tissue in order to inactivate the Trypsin. This was then followed by three $10 \mathrm{ml}$ washes with cold HBSS [++]. After the last wash with minimum media around the tissue, $100 \mu \mathrm{l}$ of $1 \mathrm{mg} / \mathrm{ml}$ DNase (Worthington) was added and incubated for 10 to 30 seconds. The tissue was then washed three times with $10 \mathrm{ml}$ Neurobasal media (GIBCO). With the last wash, $2 \mathrm{ml}$ of Neurobasal media was left behind in the falcon and the tissue was dissociated by using a $1 \mathrm{ml}$ pipette. After all the cell clumps were broken $8 \mathrm{ml}$ of Neurobasal media was added to this and centrifuged at $300 \mathrm{rpm}, 4^{\circ} \mathrm{C}$ for 5 minutes. The supernatant was again centrifuged at $1200 \mathrm{rpm}, 4^{\circ} \mathrm{C}$ for 5 minutes. The cell pellet was resuspended in warm $\left(37^{\circ} \mathrm{C}\right)$ Neurobasal media supplemented with 1X Glutamax (GIBCO), 1\% Penicillin- Streptomycin (GIBCO) and 1X B27 (GIBCO). The cell density was counted using a Neubauer chamber cell counter. The cells were plated on PLL coated coverslips at 50,000 cells per well density in a 24 well plate.

\subsection{Nocodazole assay}

Wild type mice belonging to the NMRI strain were electroporated with either pCAG-FMF-eGFP + pCAG-Cre plasmid or pCAG-FMF-eGFP + pCAG-Cre + pCAG-FMF-eGFP-Ninein plasmid cocktail at E14.5 and the embryos were harvested at E17.5. The electroporated region of the cortex was isolated, dissociated and cultured in vitro as described before. On DIV3 5mM nocodazole in Neurobasal complete medium was added to the neurons. Coverslips were harvested after 2 hours, 4 hours, 6 hours and 8 hours after treatment. The coverslips were fixed, stained for ac-tubulin and GFP and analyzed.

\subsection{GFP-EB3 + TIPs live imaging}

Sip 1 fl/wt and Sip $1^{f / f l}$ embryos were electroporated with $p C A G-C r e+p C A G-$ GFP-EB3 plasmid at E14.5. Sip1flfl embryos were also electroporated with 
$p C A G-C r e+p C A G-N i n e i n+p C A G$ GFP-EB3 plasmid cocktail. The embryos were harvested at E16.5 and the electroporated region was isolated, neurons were dissociated and cultured on FluroDish tissue culture dish. At DIV2 time series of GFP-EB3 comets were recorded using a Zeiss Spinning Disc system CXU-S1 at 1 frame per second for 200 seconds. The data was analyzed using the Kymograph ImageJ pluggin.

\section{$\underline{2.13 \text { In vitro cortical explant outgrowth assay }}$}

These experiments were performed in the lab of Dr. Linda Richards, Queensland Brain Institute, Australia. Satb2Cre;Rosa flox stop lacZ mice were time-mated (E13 or E17) and pregnant dams were anesthetized with $100 \mathrm{mg} / \mathrm{kg}$ sodium pentobarbitone, and placed on a heating pad. Embryos were removed and placed in Leibovitz's L-15 medium (Invitrogen), the brain was removed, and the neocortex dissected. Explants and collagen gels were prepared and cultured exactly as described earlier (Thomas Fothergill et al., 2013). Explants displaying poor growth (those below one standard deviation of the mean level of outgrowth for all experiments across all conditions) were eliminated from the experiment. Each explant was stained with TuJ1 immunohistochemistry as previously described (Thomas Fothergill et al., 2013) and imaged using a Zeiss upright microscope equipped with an Apotome optical sectioning device. Digital images were analysed using a Matlab (The Mathworks Inc.) program to define a line through the center of the explant (center of mass of pixels representing the explant body). The number of pixels representing neurites on either side of this line was then counted (values $U$ and D respectively). "Outgrowth" was defined as total neurite pixels $(U+D)$ divided by the total number of pixels representing the neurite body (Mortimer et al., 2009; W.J.Rosoff et al., 2004). 46-139 explants per condition were pooled across four experiments and the amount of outgrowth analyzed using an unpaired two-tailed Student's t-test. Data are presented as average values \pm standard error of the mean (SEM). 


\subsection{Organotypic slice culture and bead implantation assay}

We cut 200-250- $\mu$ m-thick sections of E16.5 Unc5C or EGFP electroporated brains using a vibratome and cultured them on cell-culture inserts $(1-\mu \mathrm{m}$ pore size, BD Biosciences) according to the protocol described previously(Polleux \& Ghosh, 2002; E Seuntjens et al., 2009). The slices were cultured in a medium consisting of complete Hank's Balanced Salt Solution, basal medium Eagle, 20 mM D-glucose, $1 \mathrm{mM}$ L-glutamine, penicillin (100 $\left.\mathrm{U} \mathrm{ml}^{-1}\right)$, streptomycin (0.1 $\left.\mathrm{mg} \mathrm{ml}^{-1}\right)$, N2 supplement (100 $\mu \mathrm{l}$ per $\left.12.5 \mathrm{ml}\right)$ and $10 \%$ heat-inactivated horse serum (vol/vol). Agarose beads (Affi-gel Blue gel, Bio-Rad Laboratories) were washed three times with sterile water and four times with PBS. For coating the beads with Netrin1, $20 \mu \mathrm{l}$ of bead solution was mixed with $10 \mu \mathrm{l}$ of a Netrin1 solution (250 ng $\mu \mathrm{l}^{-1}, \mathrm{R} \& \mathrm{D}$ Biosciences) and incubated overnight at $4^{\circ} \mathrm{C}$. BSA coated beads were used as a control for which $20 \mu \mathrm{l}$ of bead solution was mixed with $20 \mu \mathrm{l}$ of BSA $\left(1 \mathrm{mg} \mathrm{ml}^{-1}\right)$. Before placing the beads on the slices, they were washed with PBS and then resuspended in $100 \mu$ of slice culture medium. Using a mouth pipette, the beads of the desired size were picked and placed over the midline in the region where the CC crosses. The cultured slices were immunostained (see above) after three days.

\section{$\underline{\text { 2.15 Chromatin-Immunoprecipitation (ChIP) }}$}

The ChIP-IT express kit (Active Motif) was used for performing the ChIP assay, according to the manufacture's instructions. Rat monoclonal anti-Ctip2 (Abcam), Rabbit anti- Satb2 (self generated), Rabbit anti- Sip1 (self generated) and polyclonal anti-rabbit IgG (Active Motif) antibodies were used. The following primers were used for qRT-PCR:

NIN-P1 fwd: 5' CTGGTGGTGAATGTGTCCTG 3'

NIN-P1 rev: 5' TGGGCTTCATATCCATCTCC 3'

NIN-P2 fwd: 5’ ATGCATCCAGGTGTTGGTTT 3’ 
NIN-P2 rev: 5' GAGGTCAAGGCCATTCTCAG 3'

NIN-P3 fwd: 5' GGGCTAAAGAGCAGGAGGAA 3'

NIN-P3 rev: 5' CTGTCAATCTGCCAAACCAG 3'

NIN-P4 fwd: 5' ACAGCCCTGATGAAAACCAG 3'

NIN-P4 rev: 5' CTGAGCACAAGTGTGGGAGA 3'

NIN-P5 fwd: 5' TGCAGCCTGTTCTGATTGAC 3'

NIN-P5 rev: 5' CCTTTCTTGGTCCTGTGCAT 3'

Unc5C E1 fwd: 5' ATCAAGCGCAACTCCCTAAA 3'

Unc5C E1 rev: 5' CTTGCTCACTTGCTCACTCG 3'

Unc5C E2 fwd: 5' CCCTTGGAGAAAGTGGAGTG 3'

Unc5C E2 rev: 5’ GTGTACGGGGAAGGGAAAC 3’

DCC MAR1 fwd: 5' TGCACAGCACCTATGATCTTG 3'

DCC MAR1 rev: 5' AACAGAGGAGTCAGAGCGAAA 3'

DCC MAR2 fwd: 5' CGCACACACATTATTCTTTTGG 3'

DCC MAR2 rev: 5’ ACTGCCTGGCTCTGTACTCC 3'

Data are presented as average values \pm standard deviation (SD).

\section{$\underline{\text { 2.16 Luciferase Assay }}$}

The luciferase assay for Sip1 binding to Ninein promoter was done in HEK293T cells. The following primers were used for cloning the Nin-P1 region and Nin-P4 regions. The PCR products were initially cloned into pGEMT (promega) and sequence verified. Subsequently, the inserts were subcloned into pMCS-GL (Thermoscientifc).

NIN-P1 fwd: 5’ AGCTGAATTCCTTCTGAAGACATCTTGATAATACAGC 3'

NIN-P1 rev: 5' AGCTGGATCCAGGAGGAGGTAGGTCACCAGGAACA 3'

NIN-P4 fwd: 5’ AGCTACTAGTGGGGAGAGACTACACCTGCTCACA 3'

NIN-P4 rev: 5' AGCTGGATCCGGATGTCCCCCGTCTCTTAAAAGC 3' 
Fragments cloned into pMCS-GL were transfected along with pCMV-Alkaline Phosphatase in HEK293T cells using lipofectamine 2000. 48 hours post transfection, cell media was collected and used for assaying using the SecretePair Dual Luminescence assay kit (Genecopoeia). Glomax (promega) was used for measuring the luminescence. Values are represented as Ratio of Gaussia luciferase to Alkaline phosphatase luminescence ratio \pm standard deviation. Values were collected from three independent experiments, with atleast three replicates for each experiment.

The luciferase assay for Ctip2 binding Unc5C promoter was done in HEK293T cells. Putative Ctip2 binding promoter regions in the Unc5C promoter region were amplified with the following primers:

E1Fw: 5'TTAAGAATTCTTGCCTTCTTTCCCATCT 3'

E1Rv: 5'TATTGAGGATCCAGTGCTGGGAGGTGTAGCGC 3'

E2Fw: 5'TTAAGAATTCTCCTCCTCGGAGGCTC 3'

\section{E2Rv: 5'TATTGAGGATCCTGAGACGCGCAAACAGCCGA 3'}

The fragments were cloned into pC1-TK-luciferase vector (Evrogen). E1 and E2 fragments included -842 to $-124 \mathrm{nt}$ and -60 to $+65 \mathrm{nt}$ of the Unc5C 5'flanking promoter region, respectively. Lipofectamine2000 (Invitrogen) was used for transfecting cells, according to the manufacture's instructions. Transfected cells were analyzed 24 hours after transfection. Luciferase assays were done using the Dual-Luciferase kit (Promega). A mixture of plasmids coding for renilla-luciferase (RL) were used (CMV-RL, TK-RL and SV40-RL; 1:2:10 molar proportions, respectively) to account for variable transfection efficiency. Lumat LB96V reader (Berthold Technologies) was used for reading the assays. Results are shown as Renilla-normalized relative luciferase units (RLUs) \pm standard deviation (SD). Data shown is atleast from three independent experiments. All $\mathrm{p}$ values were $\leq 0,05$.

For the luciferase assay to test Satb2 binding to DCC promoter, the following primers were used for cloning the DCC MAR1 and MAR2 regions. The PCR products were initially cloned into pGEMT (promega) and sequence verified. 
Subsequently, the MAR containing inserts were sub-cloned into pMCS-GL (Thermoscientifc).

DCC MAR1 fwd: 5’ ACTACCTAGTAGAGGCAGCCACAAG 3'

DCC MAR1 rev: 5’ AGGTGAAATTTCCACCAATGTGAAT 3'

DCC MAR2 fwd: 5' AAAATGCTACCCATGTTCCAGAAG 3'

DCC MAR2 rev: 5’ ACTGCCTGGCTCTGTACTCCAAATT 3'

Fragments cloned into pMCS-GL were transfected along with pCMV-Alkaline Phosphatase in COS7 cells using lipofectamine 2000. 48 hours post transfection, cell media was collected and used for assaying using the secretePair Dual Luminescence assay kit (Genecopoeia). Glomax (promega) was used for reading the plates. Values are represented as Ratio of Gaussia luciferase to Alkaline phosphatase luminescence ratio \pm standard deviation. Values were collected from three independent experiments, with atleast three replicates for each experiment.

\subsection{Data analysis and Statistics}

All the experiments that have been quantified have been analyzed by Student's $t$ test unless otherwise mentioned using GraphPad Prism 5.0a or Microsoft Excel software. All the graphs have been plotted using GraphPad Prism 5.0a. 


\section{RESULTS}

PART I

Non-cell autonomous role of Sip1 in Corpus Callosum formation

\subsection{Commissural projections are severely altered in the Sip1}

\section{conditional knockout}

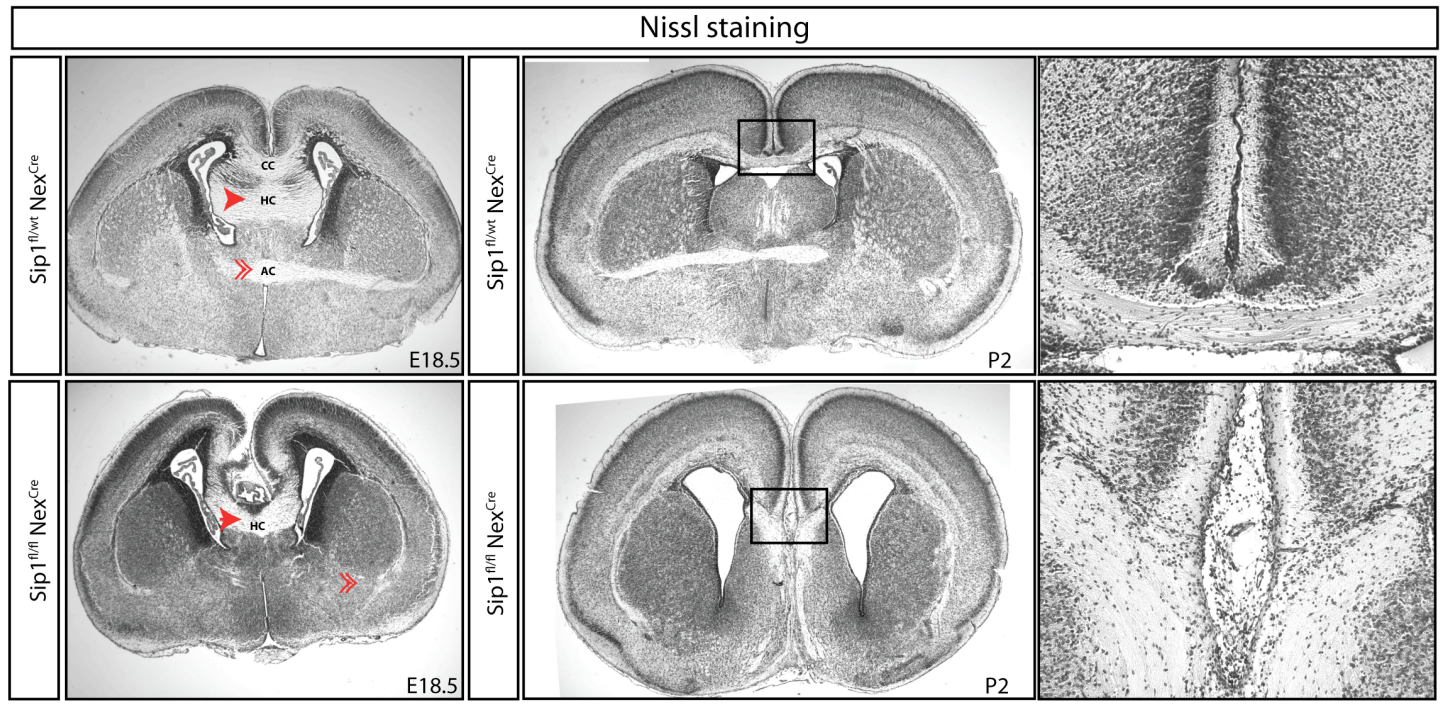

Figure 1. Cortical projections are severely altered in the Sip1 mutant

Histological analysis of the Sip1f/fl Nex Cre mutant at E18.5 through Nissl staining shows that the corpus callosum (CC) and the anterior commissure (AC) are absent, while the hippocampal commissure (HC) is unaffected. The callosal axons in the Sip $1^{f / f l} \mathrm{Nex}$ Cre mutant form Probst bundles. Higher magnification images correspond to the boxed area in the lower magnification image.

Morphological and anatomical analysis of the Sip1 mutant embryos revealed that the corpus callosum (CC) and anterior commissure (AC) were absent in the Sip1 mutant. The callosal axons failed to cross the midline and formed loose-ended bundles called Probst bundles. The AC on the other hand was completely absent. Interestingly, the hippocampal commissure (HC), which inter-connects the two hippocampi, was unaffected in the Sip1 mutant (Figure 1). 


\subsection{Cortico-fugal projections are affected in the Sip1 conditional}

\section{knockout}

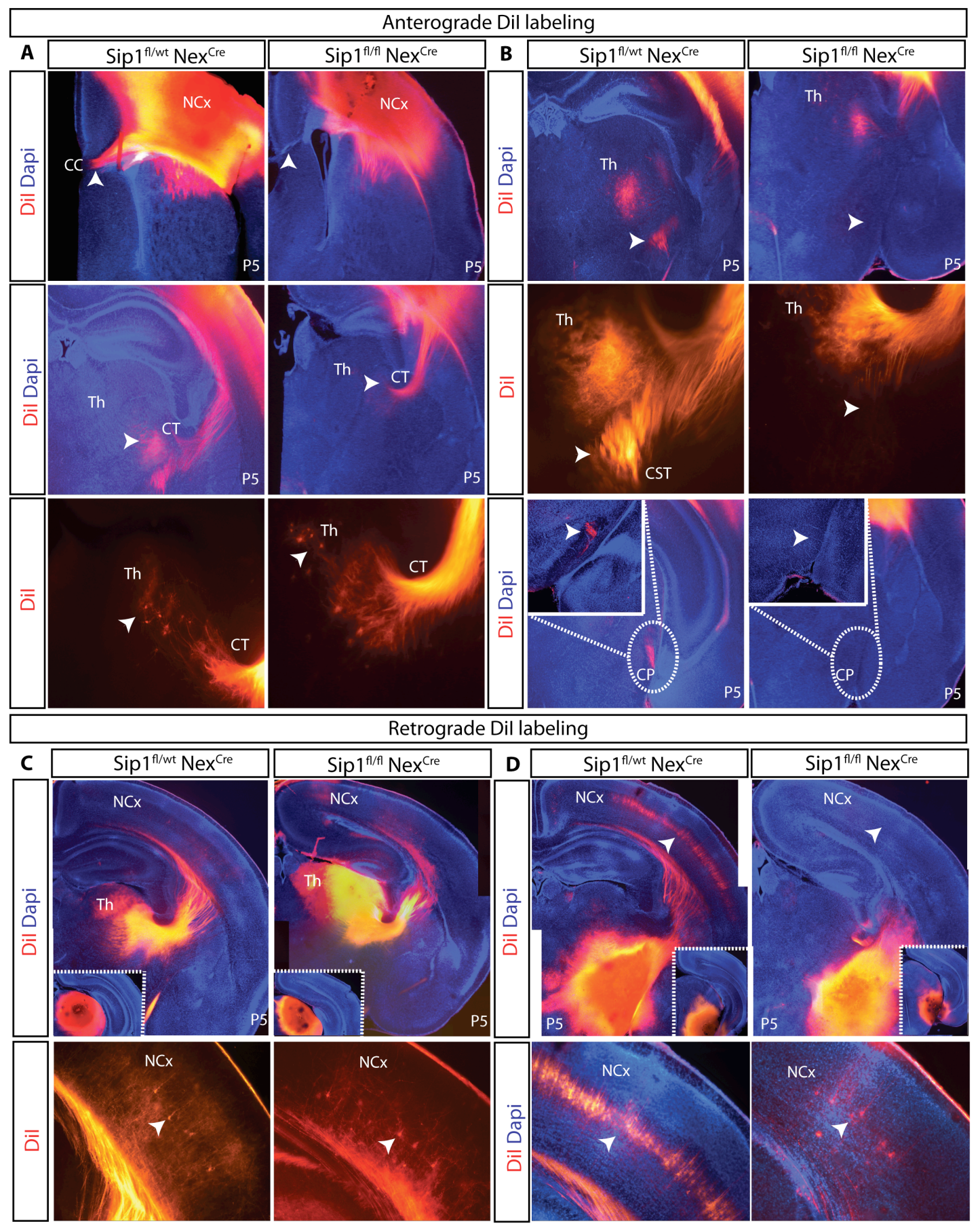

Figure 2. Commissural and corticofugal projections are affected in the Sip1 mutant

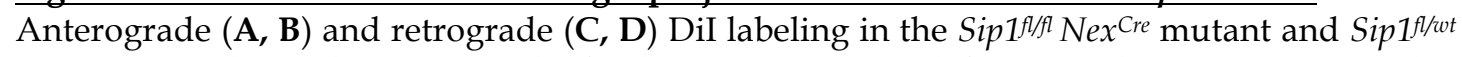
Nex Cre control, P5 brains reveals further connectivity defects in the Sip1 mutant. (A) Anterograde DiI labeling from the neocortex (NCx) confirms the absence of the corpus callosum (CC) in the Sip1 mutant. The corticothalamic tract (CT) that connects the neocortex (NCx) to the thalamus (Th) is unaffected. (B) The corticospinal tract (CST) is absent in the Sip $1^{f / f l}$ Nex Cre brains with almost no fibers passing through the cerebral peduncle (CP). (C) Retrograde DiI labeling from the thalamus shows that thalamocortical axons are unaffected in the Sip1f/flf Nex ${ }^{\mathrm{Cre}}$ brains and neurons in layer V and VI of the neocortex are back-labeled in the 
mutant and the control. (D) Retrograde DiI labeling from the cerebral peduncle confirms the absence of the CST. Very few layer V neurons are back-labeled in the Sip1 $1^{f / f} \mathrm{Nex} \mathrm{Cre}^{\mathrm{C}}$ brain when compared to Sip1f/wot Nex Cre brain. Inset boxes in $\mathbf{C}$ and $\mathbf{D}$ show the position of DiI crystal placement. Data from Master's Thesis of Srinivas Parthasarathy, IMPRS-Neuroscience Göttingen.

To further understand and characterize the changes in cortical projections as a consequence of deletion of Sip1 from the neocortex, we analyzed Sip $1^{f l / f l} \mathrm{Nex}$ Cre and Sip $1^{f l / w t}$ Nex ${ }^{C r e}$ brains at P5 using anterograde and retrograde DiI labeling. DiI (1,1'-dioctadecyl-3,3,3'3'-tetramethylindocarbocyanine perchlorate) is a lipophilic dye that is taken up by the neuronal membrane at the site of application and is transported passively through the lipid bi-layer along the axon . Placing the DiI crystals at the cell body can trace the axonal projection of the neuron and dye placement at the axonal terminal or the axonal trajectory can back-label the cell body from which the axon originates. To study the various projections from the cortex, crystals of DiI were placed in the sensory, motor and the visual cortex of fixed brains. After dye diffusion, the brains were sectioned and the axonal trajectories were analyzed. As observed earlier, the CC was absent in the Sip1 mutant. The callosal axons stopped prematurely in the ipsilateral cortex and formed Probst bundles (Figure 2A). Similar to the control brains, in the Sip1 conditional mutant, axonal bundles could be seen taking the corticofugal path and passing through the internal capsule. At the region where the corticofugal tract branches, only the dorsal branch going to the thalamus called the corticothalamic tract (CT) was present in the mutant. Axons could be followed all the way into the thalamus into the various thalamic nuclei. The presence of the thalamocortical axons (TC) could also be confirmed, as thalamic neurons were back-labeled from the cortex (Figure 2A). The ventral branch of the corticofugal tract projecting to the spinal cord called the corticospinal tract (CST) was absent in the Sip1 mutant. Examining further caudal sections revealed that no DiI labeling could be observed within the cerebral peduncle in the mutant, thus reconfirming that the CST was absent (Figure 2B). Thus in the Sip1flffl Nexcre mice, although the CT was present the CST was missing. 


\subsection{Retrograde DiI labeling reconfirms the absence of the corticospinal tract and the presence of the corticothalamic tract.}

In order to confirm the presence of the CT tract, crystals of DiI were placed in the various nuclei of the thalamus. After dye diffusion, back-labeled cells in the cortex were studied. We observed that in both the control and Sip $1^{f / f l}$ Nex Cre brains, layer VI neurons, which are known to project to the thalamus, were back labeled (Figure 2C). Similarly, to study the CST, crystals of DiI were placed in the cerebral peduncle. After dye diffusion, back-labeled cells in the cortex were studied. While in the control, layer $\mathrm{V}$ neurons were specifically labeled, in the Sip $1^{f / f l} \mathrm{Nex}$ Cre brains very few cells were backlabeled, thus confirming the absence of the CST in the Sip1 mutant (Figure 2D).

\subsection{The corticospinal tract is present at early developmental}

\section{stages in the Sip1 mutant}

In order to understand the developmental progression of the cortical projections in the Sip1 mutant we decided to study these axonal trajectories at P0. Anterograde DiI labeling from the cortex re-confirmed the absence of the CC and AC in the Sip1 $1^{\text {flfl }}$ Nex ${ }^{\text {re }}$ brain at P0. It was interesting to note that axons that project from the external capsule, which in the wild type scenario, would turn into the $\mathrm{AC}$ and project to the contralateral hemisphere, were defasciculated in the Sip $1^{f / f l} \mathrm{Nex}$ Cre brain (double arrowheads). They projected ventrally from the external capsule and could also be seen in further caudal sections of the brain unlike in the controls. Interestingly at P0, unlike at P5, the CST was present in the Sip1 mutant. Axonal projections of the CST could be traced all the way to the cerebral peduncle in the Sip1 fl/fl Nex Cre brains (arrowhead). Therefore, the CST is present at an early developmental stage (P0) in the Sip1 mutant but is lost as development progresses to P5 (Figure 3). 


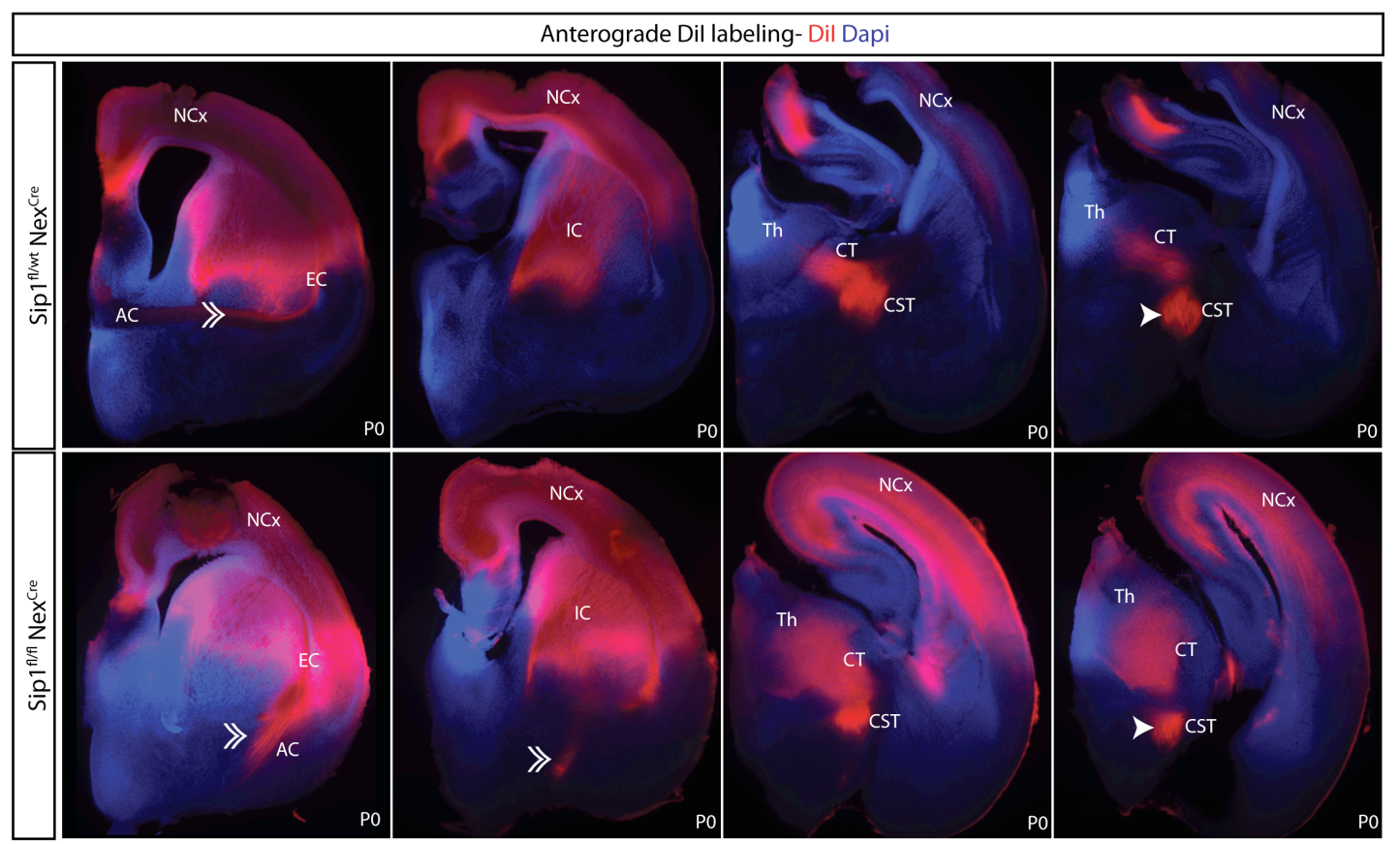

Figure 3. Corticospinal tract is present at birth (P0) in the Sip1 mutant

Anterograde cortical DiI labeling in Sip1f//wt NexCre and Sip1f/f/f Nex Cre brains at P0 shows that the anterior commissure (AC) is misrouted in the Sip1 mutant. The axons misproject ventrally instead of crossing horizontally to the contralateral hemisphere (double arrow heads). Unlike $\mathrm{P} 5$, at P0 the corticospinal tract (CST) was present in the Sip $1^{\mathrm{f} / \mathrm{fl}} \mathrm{Nex} \mathrm{Xre}^{\mathrm{Cre}}$ mutant (arrow head). External capsule (EC), Internal capsule (IC) Corticothalamic tract (CT), Neocortex (NCx), Thalamus (Th).

\subsection{Loss of corticospinal tract is not a due to cell death of Layer $\mathrm{V}$}

\section{$\underline{\text { neurons }}$}

The loss of CST could be attributed to two possible causes. Either cell death of layer $\mathrm{V}$ neurons as a result of which their axonal projections are lost or that these axons are withdrawn due to reasons like misprojection or defasciculation. In order to verify if layer $\mathrm{V}$ neurons in the Sip $1^{\text {fl/fl }} \mathrm{Nex}$ Cre brain were dying prematurely, we performed immunohistochemistry with anticleaved caspase3 antibody on sections of control and Sip $1^{f / f l} \mathrm{Nex}$ Cre brains at P2. Cleaved caspase3 is a marker for cell death. We additionally stained for Ctip2, which is a marker for layer V cortical neurons. Draq5 was used to label all the cell nuclei. The developmental stage P2 was chosen as the loss of the CST occurs between P0 and P5. 


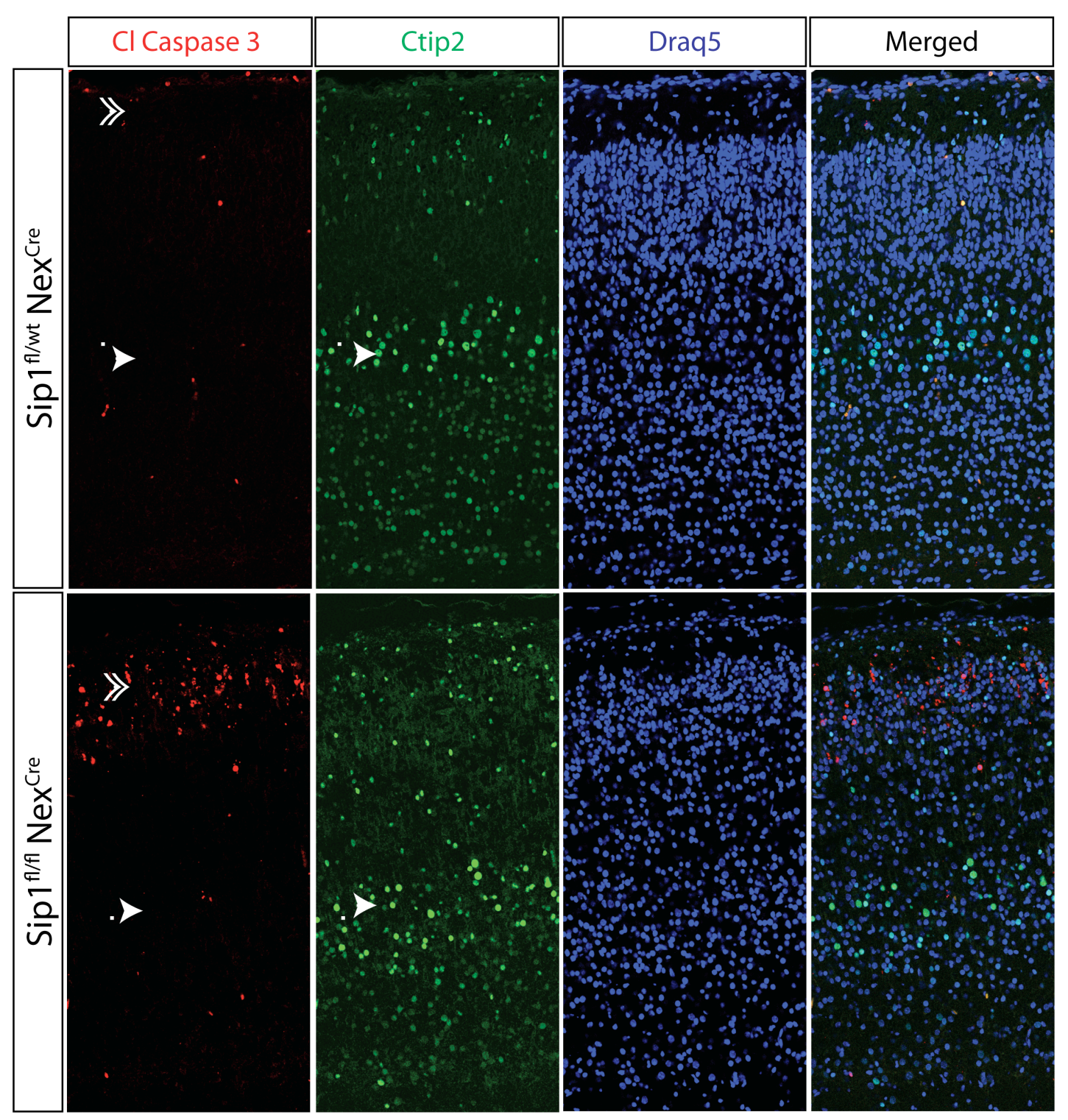

Figure 4. No apparent cell death of Sip1 deficient layer V neurons

Cleaved caspase3 staining at P2 in Sip1 $1^{f / w t} \mathrm{Nex}{ }^{\mathrm{Cre}}$ and Sip1 $1^{\mathrm{flfl} f} \mathrm{Nex} \mathrm{Cre}^{\mathrm{Pr}}$ cortex shows that there is no apparent difference in cleaved caspase 3 signal at layer V, demarcated by Ctip2 immuno labeling (arrowhead). Although, an increase in cleaved caspase3 staining in the upper layers of Sip1 deficient cortex can be seen (double arrowheads). Draq5 labels all the nuclei.

We observed that in the Sip1flfl Nexcre and control brains there was no apparent difference in cleaved caspase 3 staining in layer $\mathrm{V}$ hence ruling out the possibility of cell death of corticospinal neurons (Figure 4). Thus the loss of layer $\mathrm{V}$ projections to the spinal cord was not due to cell death but probably due to other causes. It was noted though, that there was a significant increase in cell death within the upper layers of Sip1 mutant cortex compared to the controls. 


\subsection{Midline glia is almost completely lost in the Sip1 conditional}

\section{$\underline{\text { mutant }}$}

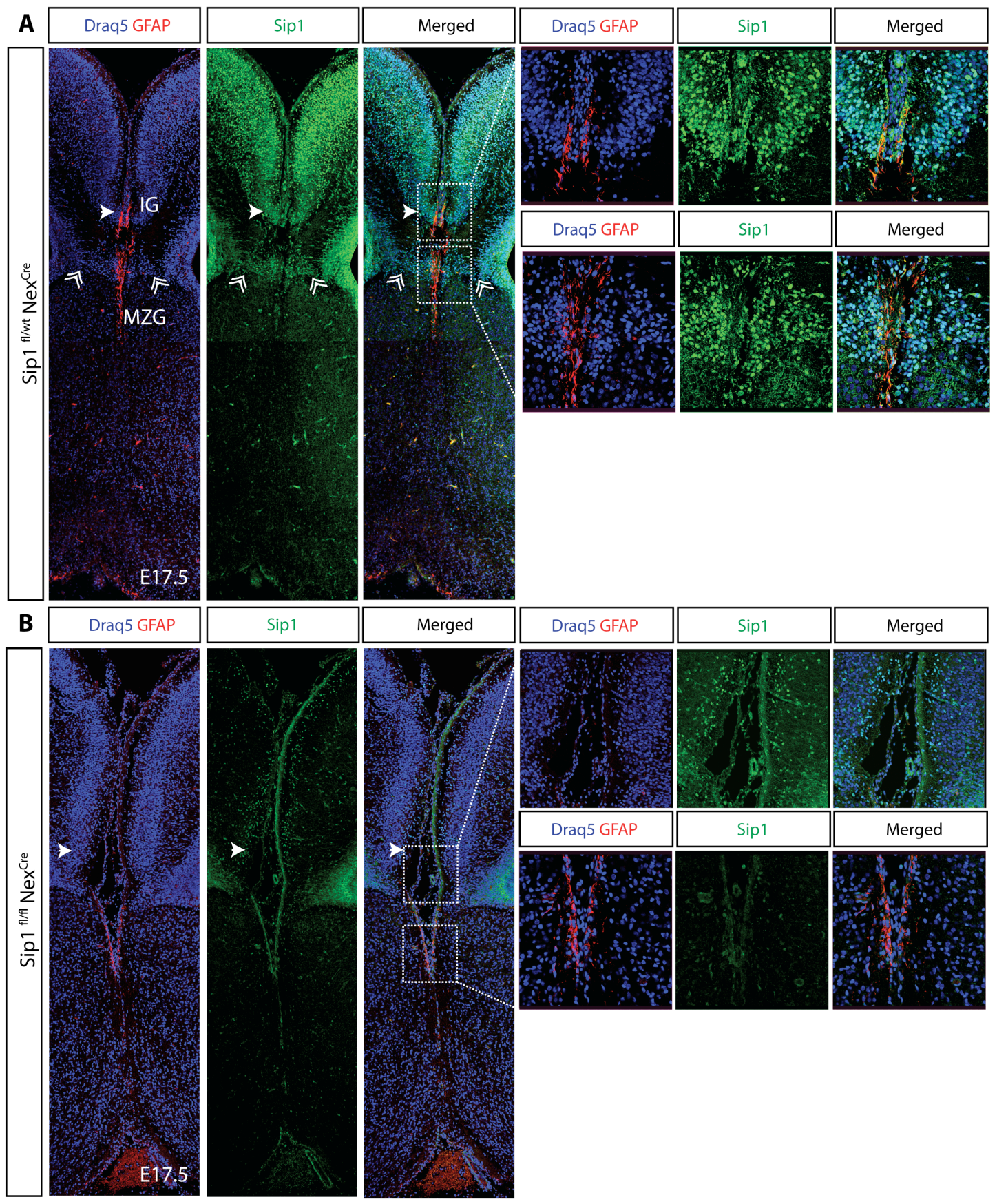

Figure 5. Midline glia is absent in the Sip1 mutant

(A) Sip1 is expressed in the GFAP positive midline glial cells in the indusium griseum (IG) and in the midline zipper glia (MZG). Sip1 is also expressed in the sub-callosal sling neurons (double arrow heads). (B) In the Sip1f/fl $\mathrm{Nex}$ Cre brains the cortical midline is not fused. GFAP positive IG glia is absent and the MZG is severely reduced. Subcallosal sling neurons are absent (double arrows in A). Higher magnification images in both panels correspond to the boxed areas in the merged images. 
GFAP positive midline glial cells have been shown to be important for guiding callosal axons. They secrete various guidance cues that bind to cognate receptors on the axons to influence axonal turning (Richards et al., 2004; Shu \& Richards, 2001; Shu et al., 2003; Silver \& Ogawa, 1983). To determine if the midline glia are intact in the Sip1 mutant, we performed immunohistological staining on control and Sip1flfl Nex Cre brain sections at E17.5 using antibodies against GFAP and Sip1. In the control brains, GFAP positive glial cells could be seen lining the cortical midline. Dorsal to the CC, GFAP positive midline glia forming the Indusium griseum (IG) could be seen. Ventral to the CC, another cluster of GFAP cells called the midline zipper glia (MZG) was present. Glial cells lining the lateral aspects of the CC called the glial wedge $(G W)$ were also visible. Sip1 was expressed in all these cell populations (Figure 5A). In contrast to the control, in the Sip1 mutant brains, the IG glia was completely lost. Although the GW and MZG glia were present, they were severely reduced. Additionally, the dorsal midline in the Sip1 mutant was not fused (Figure 5B).

\subsection{Subcallosal sling is not formed in the Sip1 conditional mutant}

Apart from the midline glial structures that help in the guidance of callosal neurons during midline crossing, previous studies have also shown the importance of subcallosal sling neurons, which can act as a source of physical as well as chemical guidance cues for callosal axons (Figure 5A- double arrowheads, Figure 6A). One population of these sling neurons includes calretinin (CR) expressing cells (Niquille et al., 2009; Shu et al., 2003). The subcallosal sling neurons were absent in the Sip1 mutant; a sling like structure could not be discerned at the midline (Figure 5B). To hence study this population of cells further, sections of E15.5 and E17.5 Sip1fl/wt Nex Cre and Sip $1^{f l f l}$ NexCre brains were stained with an antibody against CR. At E15.5, a stage before midline fusion and midline crossing of $\mathrm{CC}$ occurs; the distribution of CR positive neurons in the Sip1 mutants matched the distribution in the control brains. The CR positive cells were found to line the 
two edges of the cortical midline (Figure 6B). By E17.5 in the control brains, a stage when callosal axons have usually crossed the midline in wild type brains, CR positive sling neurons were found to line the ventral trajectory of the corpus callosum. They were also found as dispersed cells within the white matter. On the other hand, in Sip1 mutants, the CR positive neurons did not form a midline sling but instead were found as aggregates of cells lining the two edges of the midline (Figure 6C). Therefore, apart from the midline glia, another accessory guidance structure- the sling neurons, was also disrupted in the Sip1 mutant possibly contributing to the acallosal phenotype.

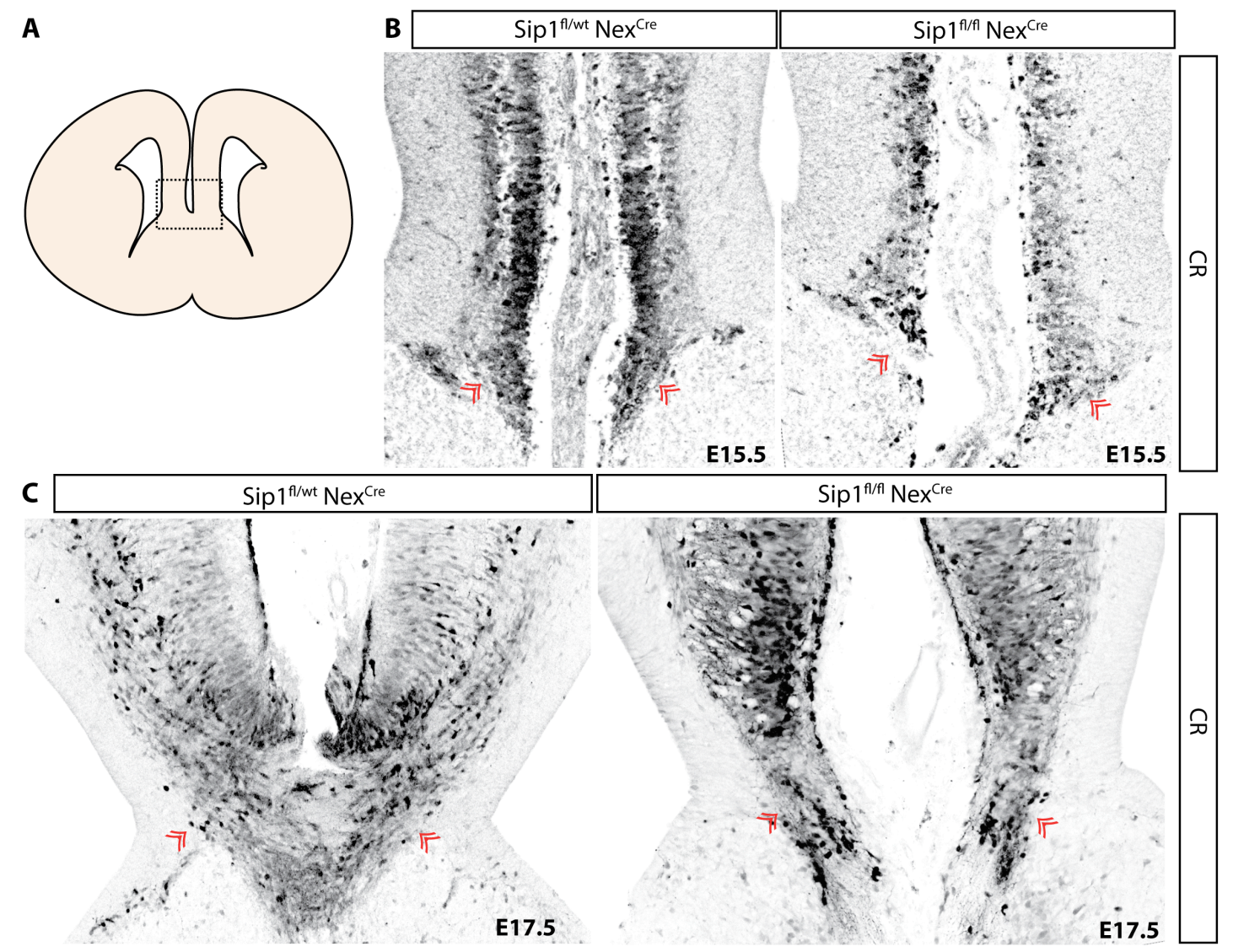

Figure 6. Calretinin positive sub-callosal sling neurons are mislocalized

(A) Calretinin (CR) positive cells form one population of sub-callosal sling neurons and are found in the cortical midline below the corpus callosum. (B) CR positive cells are similarly distributed in Sip1f//wt Nex Cre and Sip1/f/f Nex Cre midline at E15.5, a stage before CC formation. (C) At E17.5, when callosal fibers have crossed the midline, CR positive neurons can be seen forming a subcallosal sling in Sip $1^{f / / w t} \mathrm{Nex}{ }^{\mathrm{Cre}}$ brain but not in Sip $1^{f / f / f} \mathrm{Nex} \mathrm{Cre}^{\mathrm{C}}$ mutant. 


\subsection{Slit1, Sema3C and other axon guidance molecules show}

\section{altered expression levels in the Sip1 mutant}

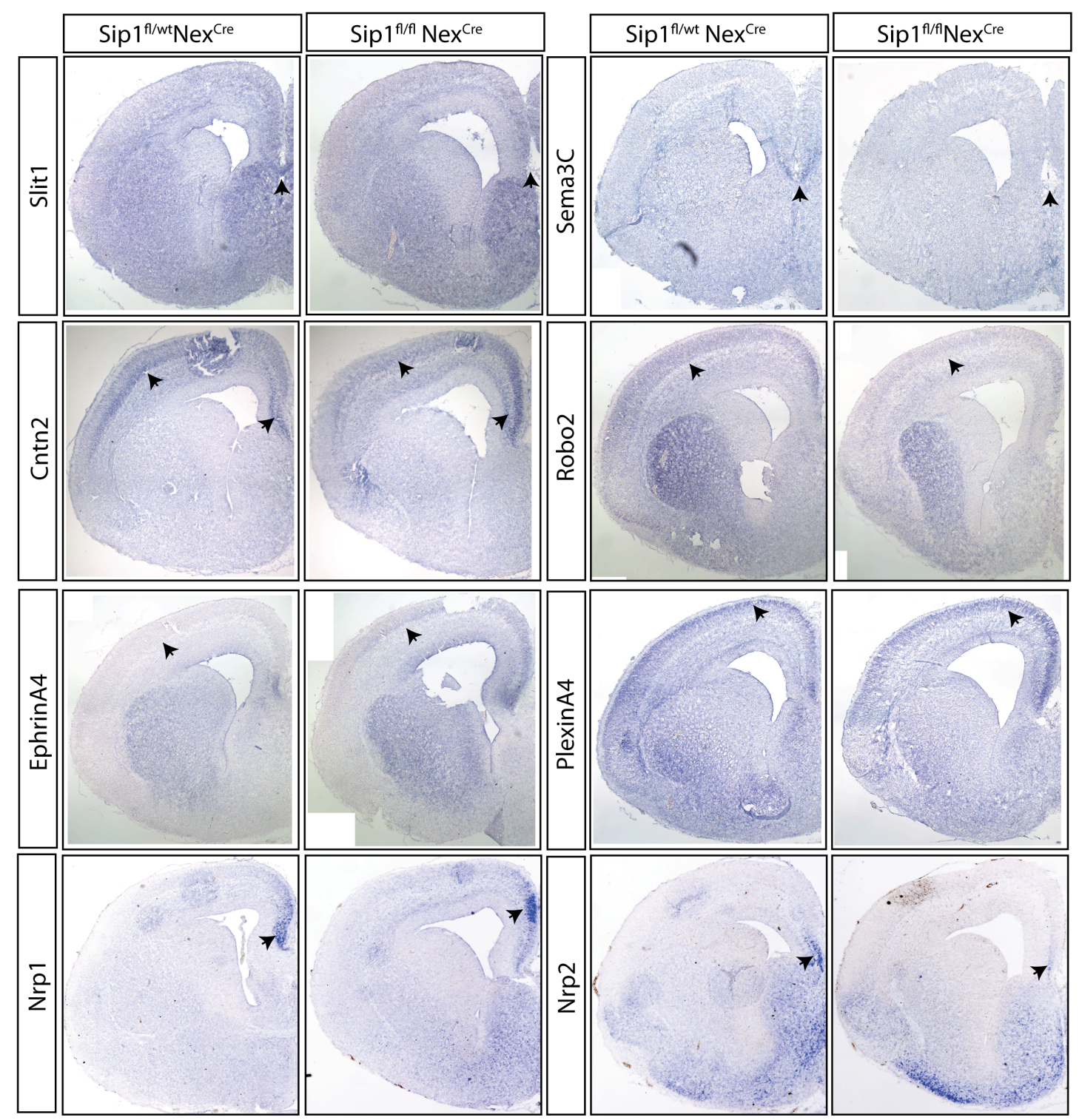

Figure 7. Many axon guidance molecules including Slit1 and Sema3C show altered expression pattern in the Sip1 mutant

In situ hybridization for different axon guidance molecules showed altered expression pattern in E16.5 Sip1 mutant sections when compared to controls. Arrowheads highlight the region of altered expression. Expression of Slit1 and Sema3C is lost at the midline. Cntn2 and Robo2 show a downregulation in the Sip1 mutant while ephrinA4 and PlexinA4 show an upregulation. Nrp1, which is expressed in the cingulate cortex, is marginally upregulated while Nrp2, which is also expressed at the midline, is downregulated in the mutant midline. 
Slit family of guidance factors are expressed by the midline glia forming the GW, IG and MZG. The callosal axons in turn express the Robo family receptors that respond to Slit family ligands, aiding in axon guidance (Shu \& Richards, 2001). Similarly the CR positive sling neurons express Sema3C that attracts Neuropillin1 (Nrp1) expressing cortical neurons (Niquille et al., 2009). Since in the Sip $1^{f / f l}$ Nex ${ }^{\text {Cre }}$ mice both the midline glia as well as the midline sling neurons were affected we wanted to investigate if the expression of Slit family molecules and Sema3C was also affected. In situ hybridization using probes against Slit 1,2,3 and Sema3C showed that there was a visible down regulation of Slit1 and Sema3C at the midline in the Sip1 mutant (Figure 7). The lack of these chemical guidance cues in addition to the change in the midline morphology could explain the absence of midline crossing in the Sip1 mutant.

Apart from the Slit family and Sema3C, we tested for changes in expression levels and patterns of other axon guidance molecules as well (Table 1). Of the many different molecules tested we found changes in expression levels of Contactin2 (Cntn2), Robo2, EphrinA4, PlexinA4, Neuropillin1 (Nrp1) and Neuropillin2 (Nrp2) in the Sip1 mutant (Figure 7). In wild type E16.5 embryos Cntn2 showed a high lateral-low medial expression gradient in the cortex. In the Sip1 mutant this gradient was reversed such that the expression level of Cntn2 was higher medially and lower laterally. While Robo2 was downregulated in the Sip1 mutant cortex, EphrinA4 was upregulated. The expression domain of PlexinA4 seemed to be expanded in case of the Sip1 mutant. Nrp1 and Nrp2 are expressed in the cingulate cortex in the wild type brain. In the Sip1 mutant while the expression of Nrp1 was marginally upregulated, the expression of Nrp2 was considerably downregulated. Thus apart from crucial midline-guidance molecules other axon guidance molecules that have been shown to be important for influencing axonal trajectory were also altered in the Sip1 mutant. 
Table 1. Axon guidance molecules tested by ISH in the Sip1 mutant

\begin{tabular}{|c|c|c|c|c|c|}
\hline Nr. & Gene & ISH result & Nr. & Gene & ISH result \\
\hline 1 & Contactin 1 & No change & 16 & PlexinA1 & No change \\
\hline 2 & Contactin2 & $\begin{array}{l}\text { Down- } \\
\text { laterally } \\
\text { and up- } \\
\text { medially }\end{array}$ & 17 & PlexinA2 & No change \\
\hline 3 & Ephrin A1 & No change & 18 & PlexinA3 & No change \\
\hline 4 & Ephrin A2 & No change & 19 & PlexinA4 & $\begin{array}{l}\text { Up- } \\
\text { regulated }\end{array}$ \\
\hline 5 & Ephrin A4 & $\begin{array}{l}\text { Up- } \\
\text { regulated }\end{array}$ & 20 & PlexinB4 & No change \\
\hline 6 & Ephrin A5 & No change & 21 & Robo1 & No change \\
\hline 7 & Ephrin B1 & No change & 22 & Robo 2 & $\begin{array}{l}\text { Down- } \\
\text { regulated }\end{array}$ \\
\hline 8 & Ephrin B2 & No change & 23 & Robo3 & No change \\
\hline 9 & EphrinB3 & No change & 24 & Slit1 & $\begin{array}{l}\text { Down- } \\
\text { regulated }\end{array}$ \\
\hline 10 & EphA2 & No change & 25 & Slit2 & Inconclusive \\
\hline 11 & EphA4 & No change & 26 & Slit3 & Inconclusive \\
\hline 12 & EphB1 & No change & 27 & Sema3A & No change \\
\hline 13 & EphB2 & No change & 28 & Sema3C & $\begin{array}{l}\text { Down- } \\
\text { regulated }\end{array}$ \\
\hline 14 & Neuropilin 1 & $\begin{array}{l}\text { Marginally } \\
\text { up- } \\
\text { regulated }\end{array}$ & 26 & Sema3F & No change \\
\hline 15 & Neuropilin 2 & $\begin{array}{l}\text { Down- } \\
\text { regulated }\end{array}$ & 27 & Sema3G & No change \\
\hline
\end{tabular}


3.9 The lack of Sema3C expression in the Sip1 mutant is a noncell autonomous effect
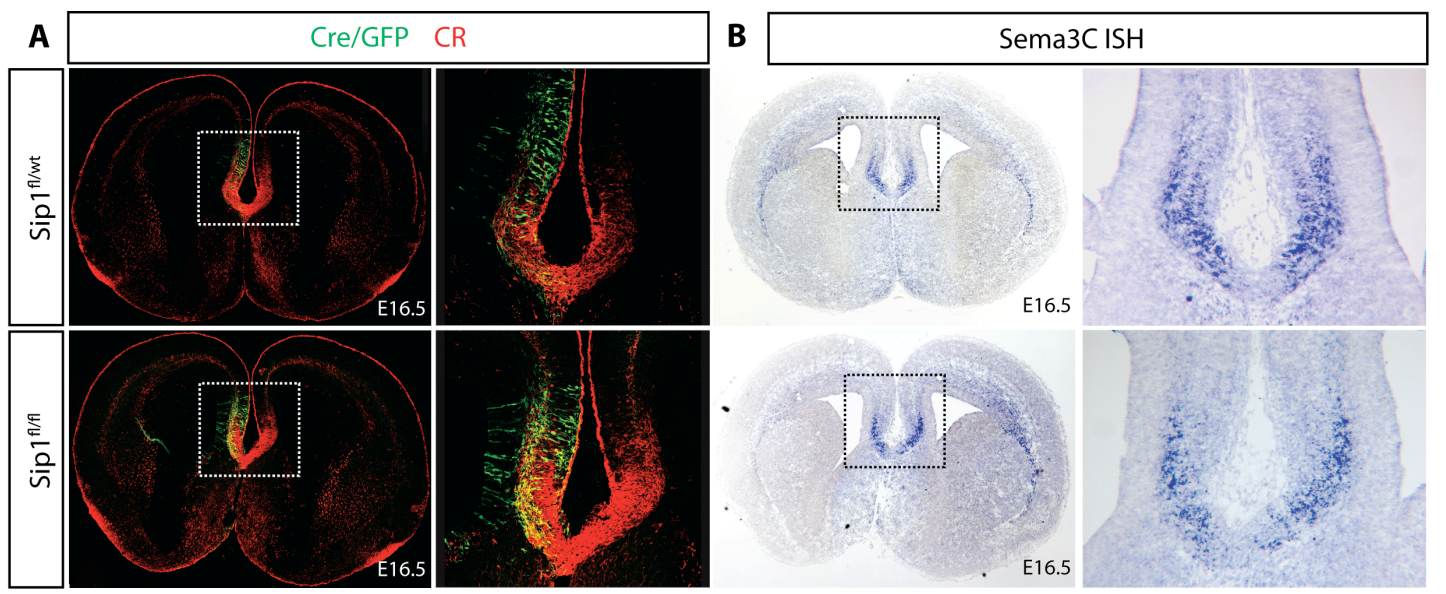

Figure 8. Loss of Sema3C expression in the Sip1 mutant is a non-cell autonomous effect

(A) In utero electroporation of GFP-IRES-Cre at the cortical midline of Sip1f/fl and Sip 1 f//wt brains does not alter the position of calretinin (CR) positive subcallosal sling neurons. (B) In situ hybridization of the adjacent sections of the electroporated brains show that the expression of Sema3C is also unaltered.

To further understand if the change in Sema3C expression levels in the Sip1 mutant is due to cell intrinsic loss of Sip1 or due to cell extrinsic effects we electroporated GFP-IRES-Cre plasmid into the cortical midline of Sip $1^{f / w t}$ and Sip $1^{f l / f l}$ embryos at E12.5. This ensured a mosaic deletion of Sip1 specifically in the electroporated cells at the midline while not affecting the rest of the brain. The embryos were harvested at E16.5; alternate sections were stained for CR and GFP (Figure 8A), while subsequent sections were processed for in situ hybridization for Sema3C (Figure 8B). We observed that despite the deletion of Sip1 from the electroporated cells in the midline, no visible changes could be seen in the distribution of CR positive cells in the Sip1flfl brain (Figure 8A). Sema3C probe signal was also comparable to the control electroporation (Figure 8B). Hence the loss of Sema3C expression in the Sip1 mutant midline is a non- cell autonomous effect of Sip1 deletion. 


\section{$\underline{3.10 \text { Sip1 mutant shows altered midline morphology }}$}

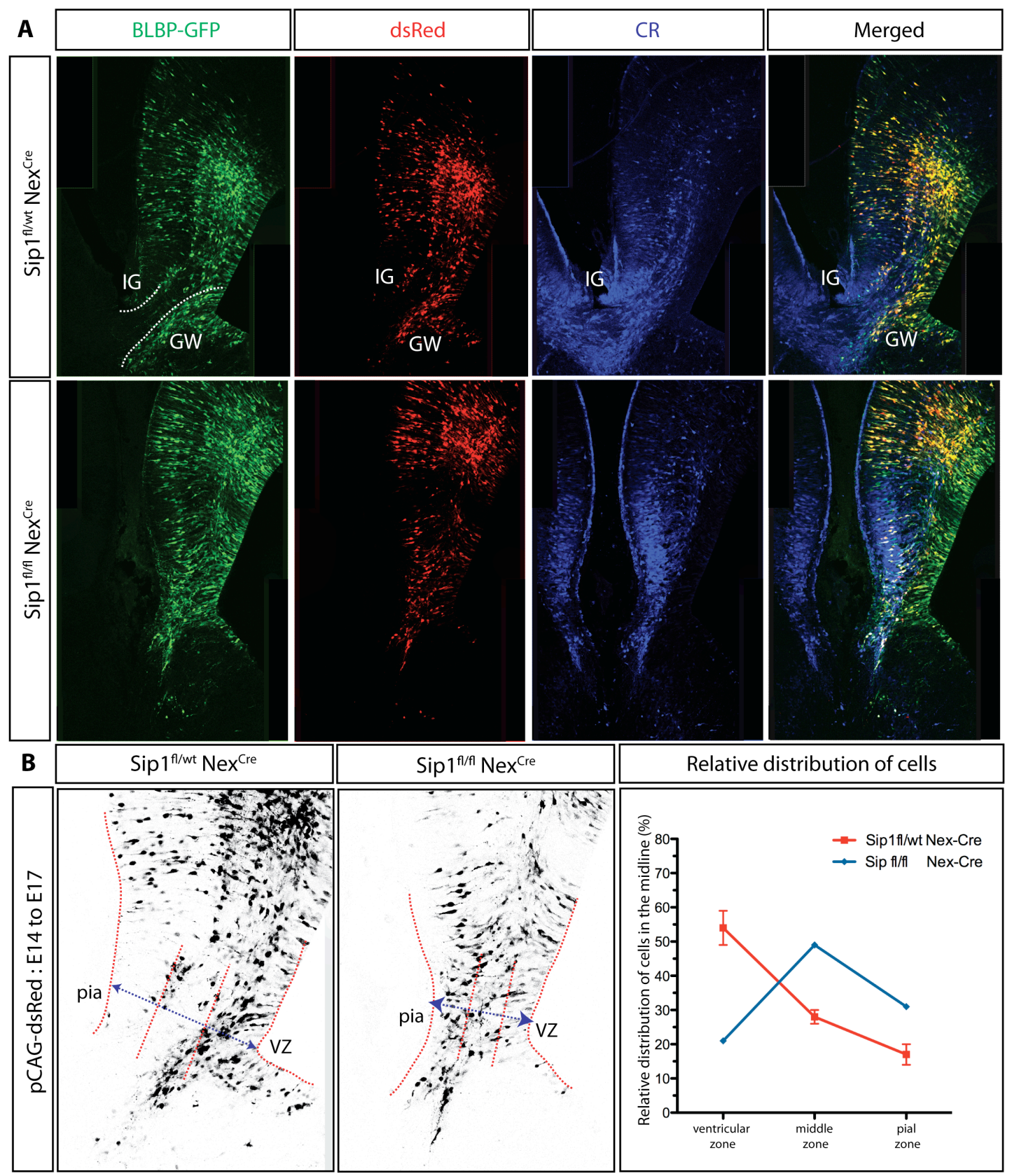

Figure 9. Sip1 mutant midline is narrower and shows an altered distribution of cell density (A) In utero electroporation of pBLBP-GFP together with pCAG-dsRed and immuno staining for calretinin shows that the relatively cell free region in the midline that corresponds to the corpus callosum in Sip1f/wt Nex Cre is replaced by a cell dense midline in Sip1 ff/fl Nex Cre brains. Calretinin (CR) positive neurons populate these cell dense regions in the Sip1 mutant instead of forming a sub-callosal sling. (B) The cortical midline of Sip $1^{f / f l} \mathrm{Nex} \mathrm{Cre}^{\mathrm{re}}$ brains is significantly narrower than control brains (arrow) and shows altered distribution of cell density within the three equally sized bins.

To allow for callosal axons to traverse through the midline, elborate reorganization of the midline takes place (Smith et al., 2006). The cortical 
midline of wild type brains were electroporated at E14.5 with pBLBP-GFP plasmid, which labels radial glial cells and pCAG-dsRed, which labels the progenitor and postmitotic cells and analyzed at E17.5. As described previously (Shu et al., 2003; Smith et al., 2006), this experiment revealed that the BLBP positive cells present at the midline either retracted their apical process and migrated towards the pia forming the IG glia or retracted their basal process and migrated towards the ventrical forming the GW. This essentially left a cell free region in between the two populations for the callosal axons to pass through. The pattern formed by the dsRed positive cells also followed a similar arrangement (Figure 9A). On the other hand, in the Sip1 mutant brains, at the midline neither the BLBP-GFP positive glial cells nor dsRed cells separate into two populations (Figure 9A). Quantifying the relative distribution of dsRed cells at the midline within three equally spaced bins in the wild type and the mutant further reflected this difference (Figure 9B). Apart from the relative distribution of cells at the midline it was also apparent that the Sip1 mutant midline was much narrower than the wild type (Figure 9B).

\subsection{Altered distribution of neurons at the midline is a non- cell autonomous effect of Sip1 deletion}

To further establish non-cell autonomous dependence of neurons on Sip1 for the distribution at the midline, we electroporated Sip $1^{f / f l}$ embryos at E14.5 with a plasmid called pCBA-FLEx together with a plasmid encoding Cre and harvested the embryos at E16.5. The pCBA-FLEx plasmid uses the Cre- loxP system to transcribe tdTomato in the absence of Cre and transcribe eGFP on Cre mediated recombination (Figure 10B). Hence using this plasmid and varying the concentration of the Cre plasmid used, we could, in parallel, study Cre positive, hence Sip1 negative- GFP positive cells as well as Cre negative, hence Sip1 positive - tdTomato positive cells within the same cortical section (Figure 10A). The tdTomato positive cells thus served as an internal control for this experiment. To compare the distribution of 
electroporated cells at the midline, the cortical midline was divided into three equally sized bins and the relative distribution of the two different cell populations within each of the bins was examined. We observed that there was no difference in the relative distribution of the two kinds of cell populations, suggesting a non-cell autonomous dependence on Sip1 (Figure 10C).

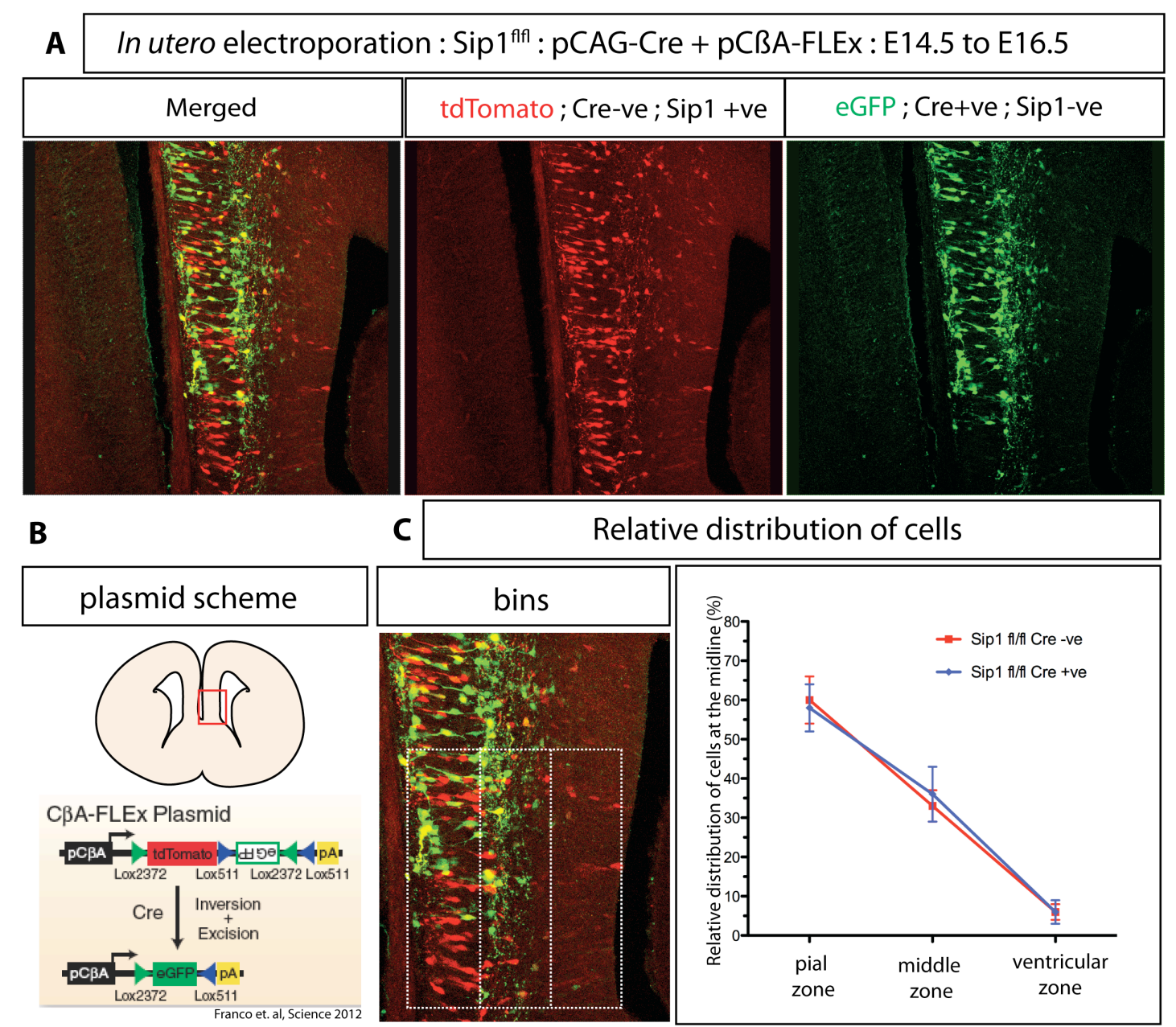

Figure 10. Change in relative distribution of cell density at the midline is a non-cell autonomous effect of Sip1 deletion

(A) In utero electroporation of $p C \beta A-F L E x$ plasmid and Cre at the midline of Sip 1 fl/fl embryos (B) from E14.5 to E16.5 resulted in a mosaic pattern of tdTomato positive/ Cre negative/ Sip1 positive cells, interspersed with eGFP positive/ Cre positive/ Sip1 negative cells. (C) Relative distribution of Sip1 positive and Sip1 negative cells within three equally sized bins drawn at the midline was measured. Both cell populations show a similar distribution at the midline. 
Taken together these experiments indicate that, the cortical midline in the Sip $1^{f / f l}$ Nex ${ }^{C r e}$ mutant mice is severely altered when compared to the wild type. The midline glia as well as the midline sling neurons that help in callosal axon guidance are absent and do not express the required guidance cues. The dorsal midline does not fuse and shows an altered morphology. These changes in the midline are probably due to non-cell autonomous effects of Sip1 deletion, as the cell intrinsic deletion of Sip1 from theses cells does not recapitulate the phenotype. 


\section{PART II}

\section{Cell autonomous role of Sip1 in establishing cortical}

\section{projections}

\subsection{Cell autonomous defects also contribute to the acallosal}

\section{phenotype in the Sip1 mutant}

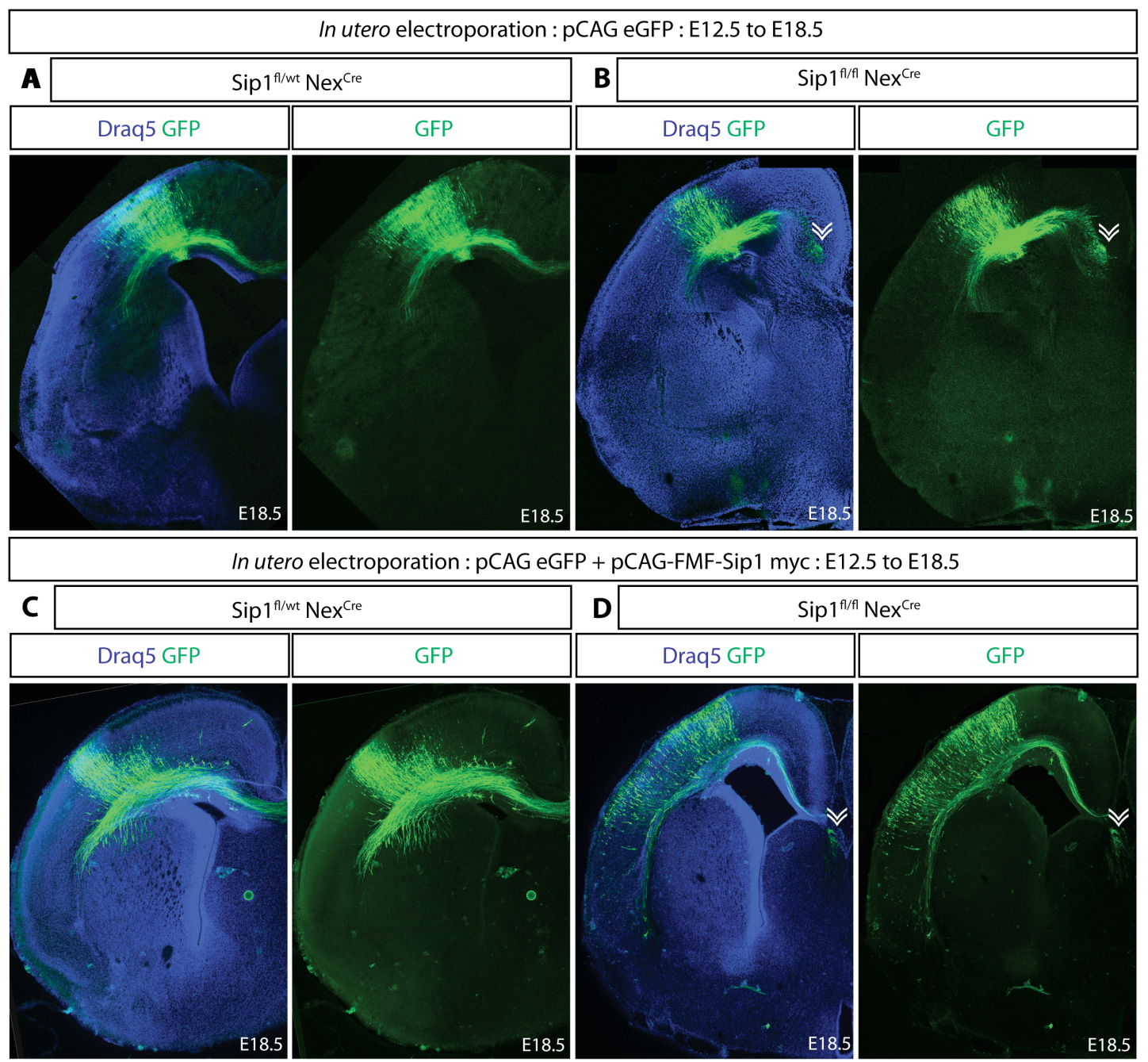

Figure 11. Acallosal phenotype in Sip1 mutant is due to both cell-intrinsic as well as cellextrinsic effects

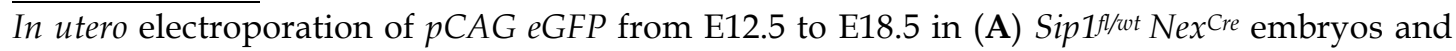
in (B) Sip $1_{f / f l}$ Nex Cre embryos shows that the GFP positive axons in Sip1fl/fl NexCre brains do not form a corpus callosum. The fibers stall medially before reaching the midline (arrowhead). In utero electroporation of $p C A G$ eGFP + pCAG-FMF-Sip1myc expressing plasmids from E12.5 to E18.5 in (C) Sip1fl/wt NexCre embryos and in (D) Sip1fl/fl NexCre embryos. The Sip1 positive/ GFP positive axons within the Sip1 mutant, do not cross the midline but yet project much further till the midline fissure, when compared to eGFP electroporation in Sip1flfll Nex Cre embryos (arrowheads in B, D). (A, C) show normal corpus callosum formation. 
Although the midline of the Sip1 mutant had numerous defects that prevent midline crossing of callosal axons we wanted to determine if these non-cell autonomous defects in the Sip1 mutant were the only cause for the lack of corpus callosum formation. If this were the case then when Sip1 is reexpressed in a subset of callosal neurons in the Sip1 mutant brain, they should continue to form Probst bundles and not form the CC. To test this we electroporated a plasmid expressing Sip1 together with eGFP into Sip1ft/wt NexCre and Sip1 flffl NexCre embryos at E12.5 and also electroporated eGFP alone into Sip1fl/wt Nex Cre and Sip1fl/fl NexCre embryos at E12.5 as controls. The brains were harvested at E18.5. eGFP expressing neurons in the Sip1 $1^{f / w t}$ Nex Cre brains projected axons to the contralateral cortex forming a CC (Figure 11A), whereas eGFP-expressing neurons in the Sip1flffl Nexcre brains ended prematurely in the ipsilateral hemisphere (Figure 11B). Electroporation of Sip1 together with eGFP in Sip1 $1^{f l f l}$ Nex Cre brains on the other hand did not look similar to the Sip $1^{f / f l}$ Nexcre brains where eGFP alone was expressed. Here, although axons did not completely cross the midline to reach the contralateral hemisphere, they were still able to navigate further, till the midline fissure (Figure 11D). Expressing Sip1 and eGFP in Sip1 $1^{f / w t}$ Nex ${ }^{\text {Cre }}$ brains resulted in a normally formed CC (Figure 11C). Thus these experiment put together showed that although the defects in the Sip1 mutant at the midline were definitely responsible for the lack of formation of the $\mathrm{CC}$, there might be other cell intrinsic defects in the Sip1 mutant, which contribute towards the acallosal phenotype.

\subsection{Mosaic deletion of Sip1 is achieved by electroporation of Cre recombinase}

To test the cell-autonomous role of Sip1 in axonal extension and CC formation it was important to study Sip1 deficient neurons and their axons within a wild type background. We hence used a plasmid expressing Cre recombinase under a ubiquitously expressed promoter following a GFP-IRES sequence. The GFP-IRES ensured that all the cells expressing Cre would also express 
GFP. The GFP-IRES-Cre plasmid was electroporated into Sip $1^{f / w t}$ and Sip $1^{f / f l}$ embryos at E12.5 and the brains were harvested at E18.5. The sections were stained for GFP and Sip1. All the cells expressing GFP and hence Cre in the Sip $1^{f / f l}$ embryos were negative for Sip1 expression while the electroporated cells in the Sip1 $1^{f / w t}$ brain were unaffected (Figure 12). Hence mosaic deletion of Sip1 could be achieved with the expression of a GFP-IRES-Cre plasmid.

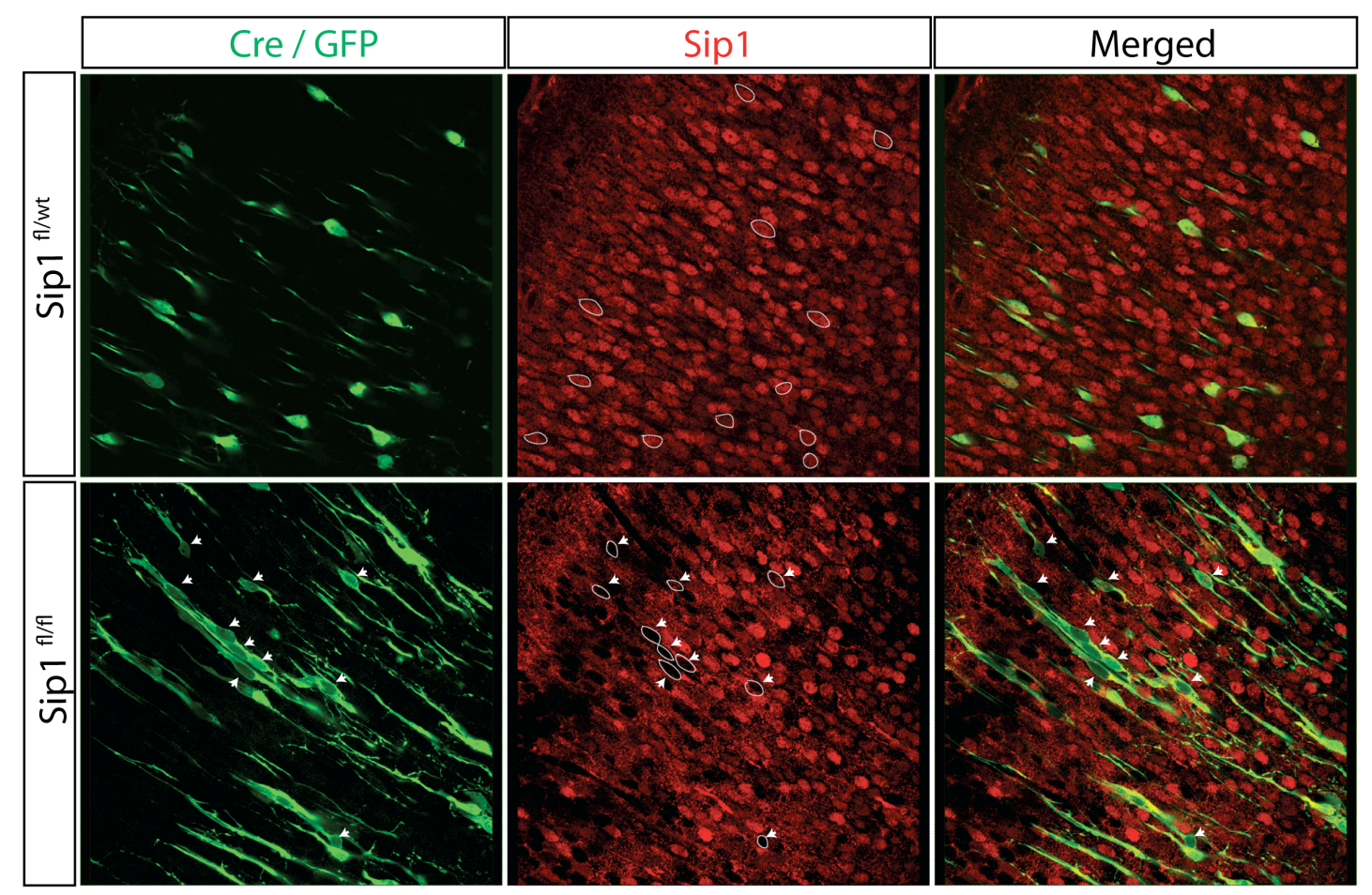

Figure 12. Cell autonomous deletion of Sip1 through in utero electroporation of Cre In utero electroporation of a GFP-IRES-Cre plasmid at E12.5 and immunohistochemistry with anti-GFP and anti-Sip1 antibody at E18.5 in Sip1f//wt and Sip1f/fl embryos demonstrates the mosaic deletion of Sip1 in Sip $1^{f / f l}$ embryos expressing GFP/Cre (arrowheads and outlines).

\section{$\underline{3.14 \text { Cell intrinsic deletion of Sip1 also affects CC formation }}$}

A GFP-IRES-Cre plasmid was electroporated into the sensory-motor cortex of Sip $1^{f / / w t}$ and Sip $1^{f l / f l}$ embryos at E12.5 and analyzed at E18.5. The sections were stained of GFP and L1 (anti-neuronal cell adhesion factor that labels axons). While the Cre/GFP positive axons in the Sip1fl/wt sections could be traced forming the CC and projecting all the way into the contralateral hemisphere, the Cre/GFP positive, hence Sip1 negative, axons in the Sip1flfl embryos did 
not form the CC Almost no GFP positive fiber could be detected at the midline crossing (Figure 13A).
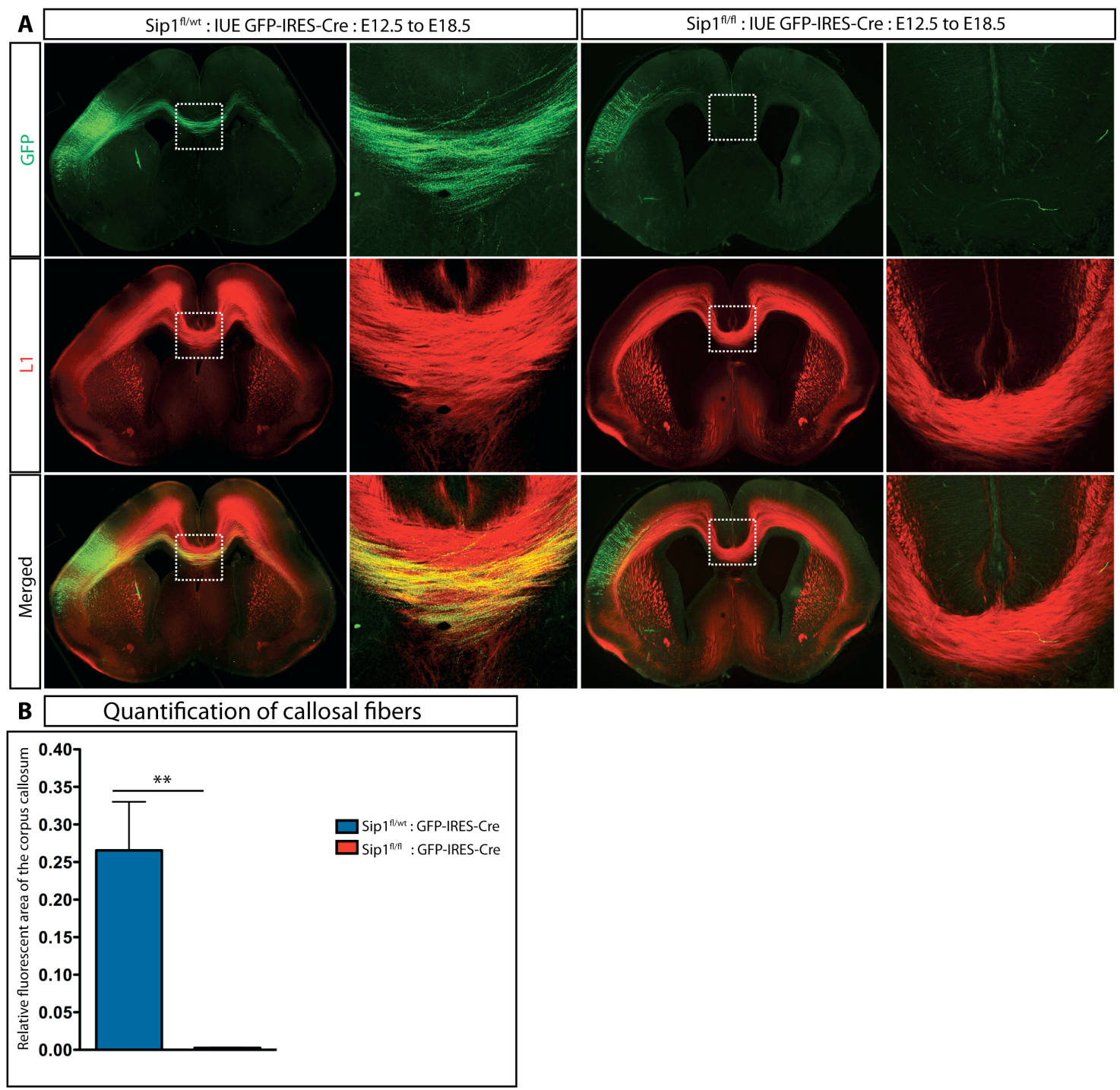

Figure 13. Callosal fibers fail to cross the midline on cell autonomous deletion of Sip1 (A) In utero electroporation of GFP-IRES-Cre at E12.5 to E18.5 in Sip1 ${ }^{f / / w t}$ and Sip1 fl/fl embryos shows that GFP positive/Cre positive /Sip1 positive axons in Sip1f/wt embryos cross the midline while GFP positive/Cre positive/Sip1 negative axons in Sip1fl/fl embryos do not cross the midline. (B) Quantification of the corpus callosum thickness at the midline relative to the electroporated cells shows severe loss of callosal fibers in Sip1flfl embryos when compared to Sip $1^{f / w t}$ embryos. Sip $1^{f / w t}=0.2656 \pm 0.06457$, Sip $1^{f l / f l}=0.0025 \pm 0.0005, \mathrm{p}$ value $=0.002, \mathrm{n}=3$.

Quantifying the thickness of the callosal axons crossing the midline, relative to the number of cells electroporated showed a $98 \%$ decrease in Sip $1 / f / f$ embryos when compared to Sip1f/wt (Figure 13B). All values represent the mean of the fluorescent area of the callosal midline relative to the fluorescent 
area of the cells electroporated in the cortex \pm standard deviation. Sip $1^{f / / w t}=$ $0.2656 \pm 0.06457, \operatorname{Sip} 1^{f l f l}=0.0025 \pm 0.0005, p$-value $=0.002, \mathrm{n}=3$.

3.15 Callosally projecting neurons within the cingulate cortex are also affected by Sip1 deletion

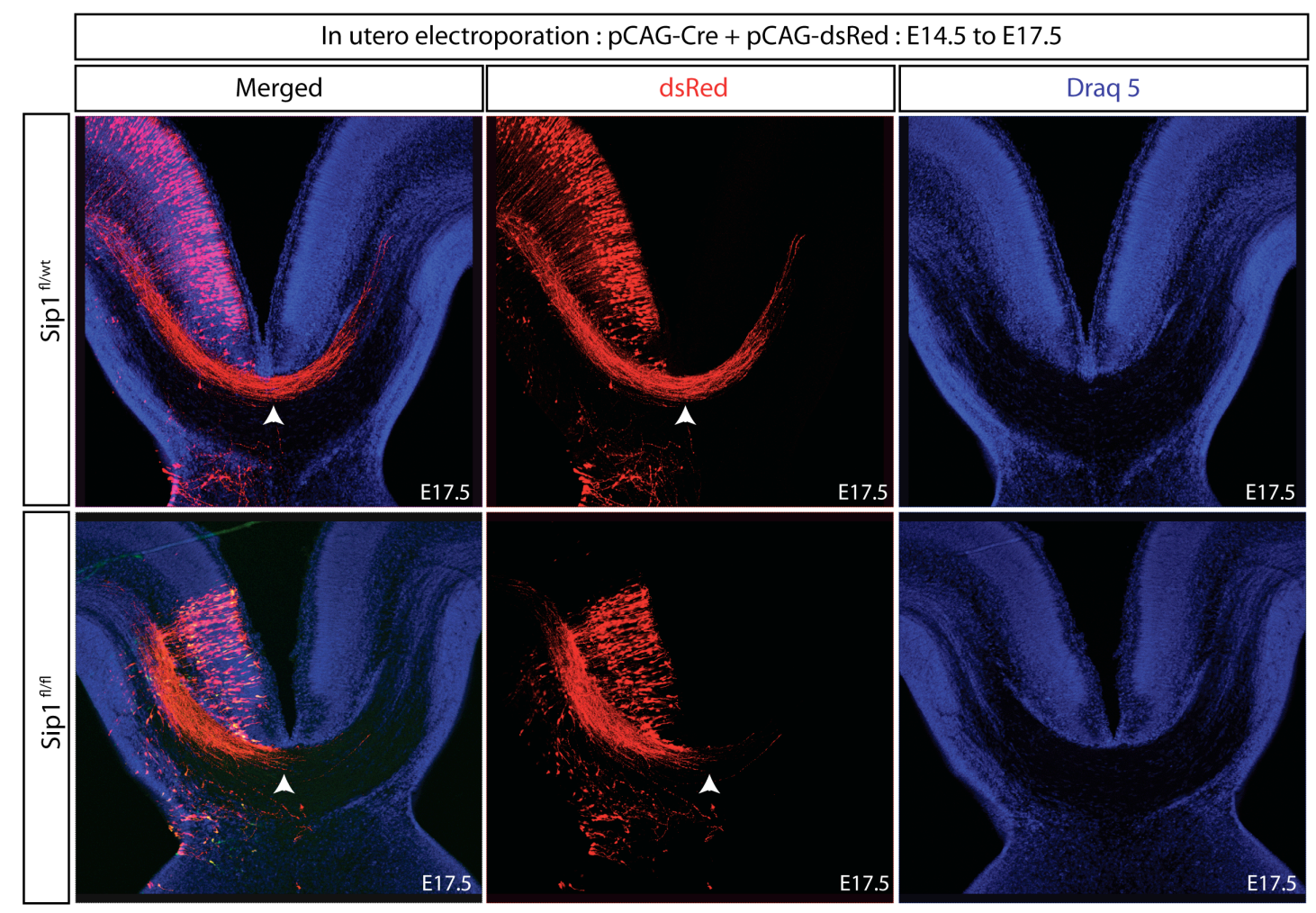

Figure 14. Projections of cingulate callosal axons are also affected due to cell intrinsic deletion of Sip1

In utero electroporation of plasmids expressing Cre and dsRed at E14.5 in Sip1 $1^{\mathrm{f} / w t}$ and Sip1/f/fl embryos at the midline show that axons projecting from the cingulate cortex do not cross the cortical midline in the Sip $1^{f / f l}$ brains at E17.5 unlike Sip $1^{f / / w t}$ brains where midline crossing can be seen (arrowheads).

The cingulate cortex, which is the medial most region of the neocortex, houses neurons that form the pioneers of the CC. They are the first set of neurons to cross the midline and aid in the crossing of the follower axons to the contralateral cortex. They take a dorsal path in crossing the midline, and the axons from the more laterally placed neurons in the cortex take a ventral path (deAzevedo et al., 1997; Koester \& O'Leary, 1994). The cingulate pioneer neurons hence have very different growth cone dynamics, speeds of axon 
extension and sometimes respond to a different set of guidance cues (G. J. Kim et al., 1991). In order to understand if these cingulate pioneer neurons also require Sip1 for correct axon extension and CC formation we injected Crerecombinase together with $d s R e d$ into the lateral ventricles of E14.5 Sip $1^{f / f l}$ and Sip $1^{f / w t}$ embryos and electroporated the cingulate cortex. The brains were harvested at E17.5. We observed that axons of the electroporated neurons in the Sip $1^{f / w t}$ projected into the contralateral hemisphere. Sip1 deficient neurons, on the other hand were incapable of extending their axons much beyond the ipsilateral white matter with very few axons managing to reach the midline (Figure 14). Hence both the pioneers as well as the follower axons require Sip1 cell intrinsically in order to form the CC.

\subsection{Cell autonomous deletion of Sip1 slows the rate of axon extension}

In order to determine if the lack of CC formation at E18.5 in the Sip $1^{f l / f l}$ brains with Cre electroporation was due to a delay in axon extention, we electroporated GFP-IRES-Cre plasmid into Sip $1^{f / w t}$ and Sip1 fl/fl embryos at E12.5 and harvested the brains from postnatal day 7 pups. We observed that callosal axons could be seen forming the CC and crossing over to the contralateral hemisphere in the Sip1 $1^{f / w t}$ as well as in the Sip $1^{f / f l}$ brains at P7 (Figure 15). Thus Sip1 deficient neurons show an axon extension defect wherein axons grow at a much slower rate when compared to their wild type counterparts. 


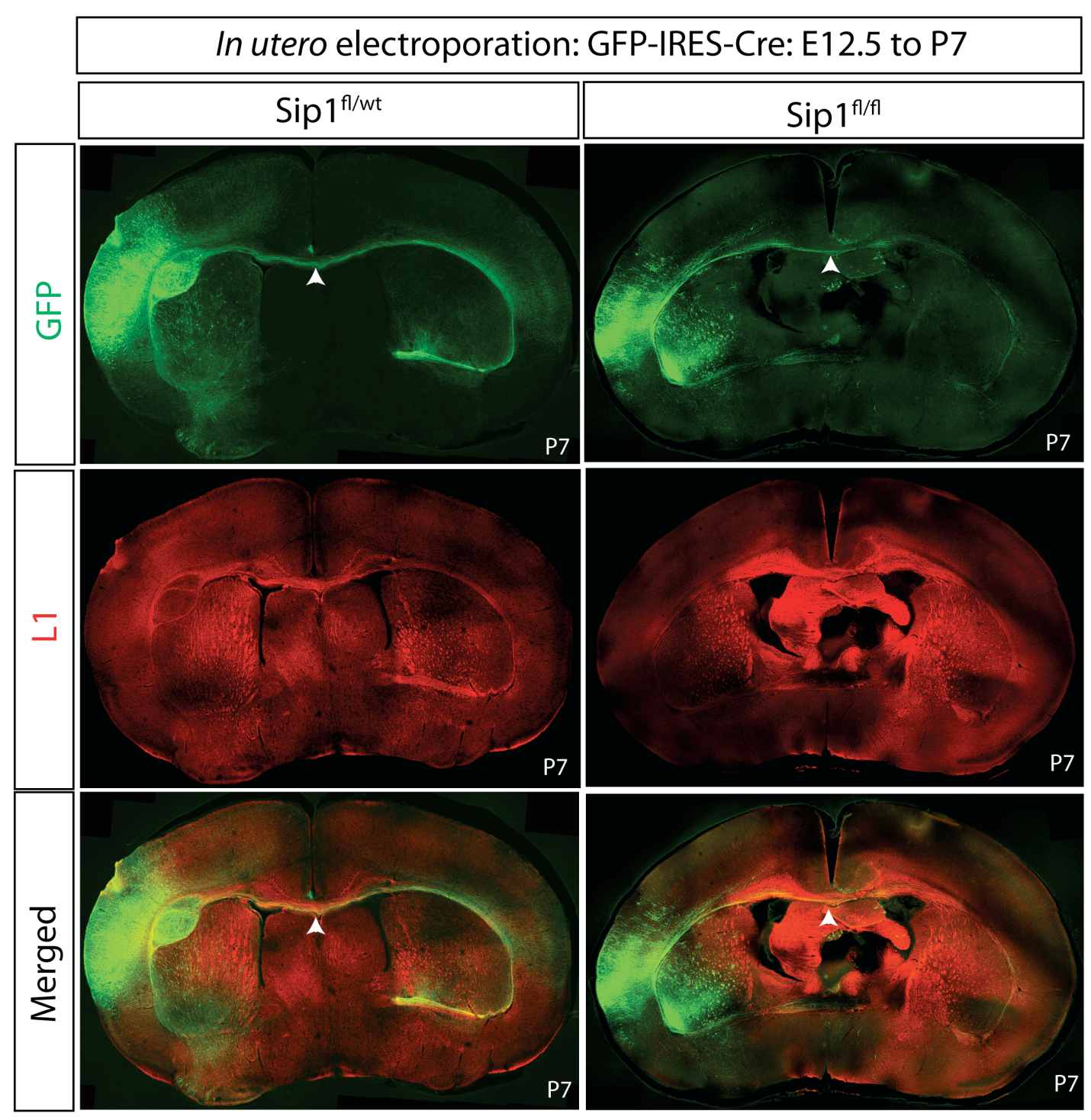

Figure 15. Lack of corpus callosum crossing with cell autonomous deletion of Sip1 is due to delay in axon extension

In-utero electroporation of GFP-IRES-Cre in Sip1f/wt and Sip1 1f/f brains at E12.5 and analyzed at P7. Immunofluorescence labeling with GFP and L1. The GFP positive axons form the corpus callosum in both Sip $1^{f / w t}$ and Sip $1^{f / f l}$ brains with axons crossing the midline (arrowheads).

\section{$\underline{3.17 \text { Sip1 deficiency affects ipsilateral axon-collateral formation }}$}

Upper layer neurons, apart from projecting to the contralateral cortex through the CC and connecting with contralateral neurons, also form axonal branches and hence synaptic connections with ipsilateral neurons. One such characteristic ipsilateral connection is formed by the axons of the upper layer 
neurons on ipsilateral layer $\mathrm{V}$ neurons through the formation of axonal collaterals (Burkhalter, 1989; Harwell et al., 2012; Thomson \& Lamy, 2007).

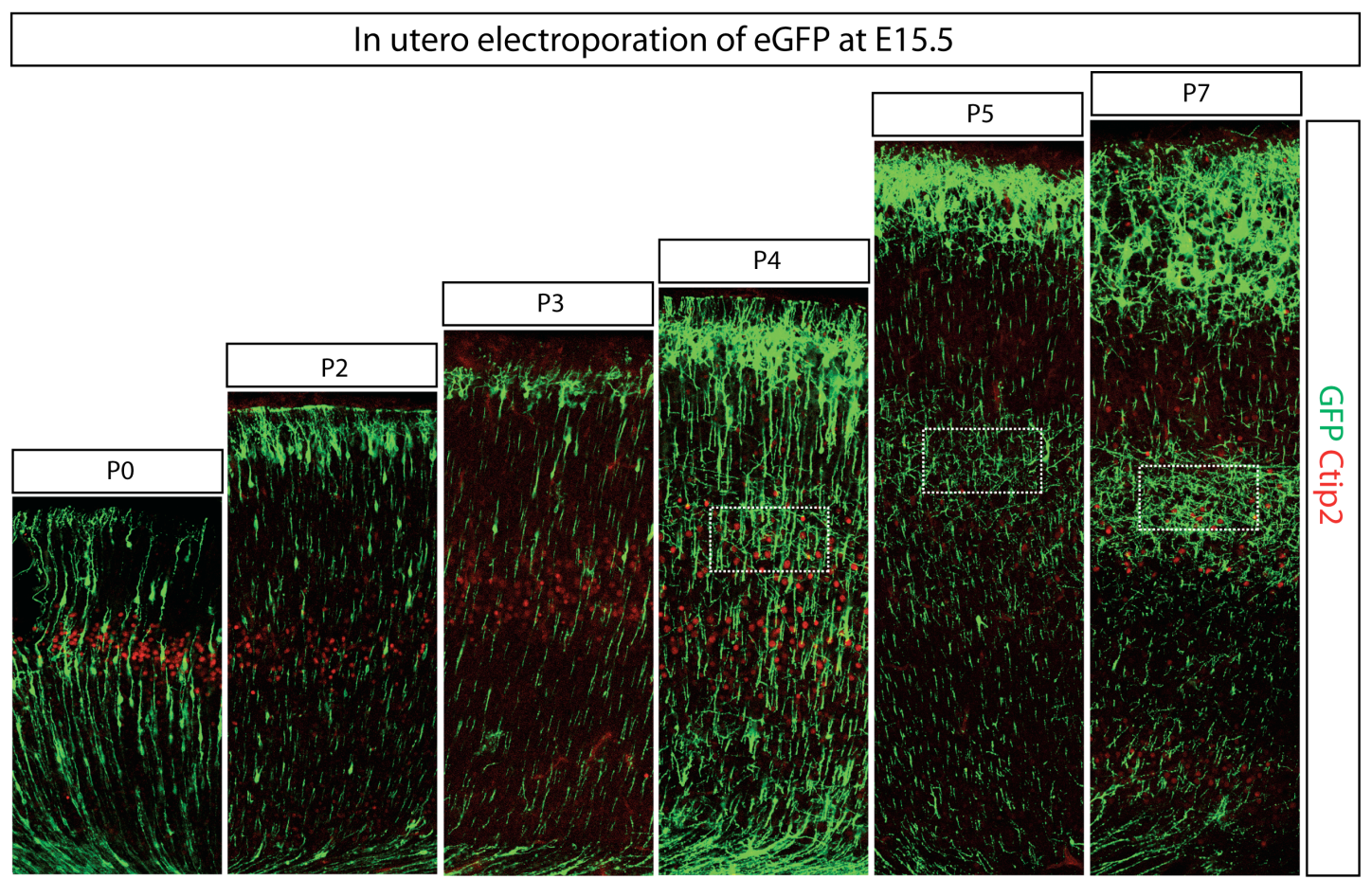

Figure 16. Upper layer neurons commence the formation of ipsilateral axonal collaterals at the level of layer $V$ neurons at $P 4$

In utero electroporation of eGFP at E15.5 in wild type embryos and subsequent immuno staining of cortical sections at P0, P2, P3, P4, P5, and P7 with Ctip2. Axons of upper layer neurons do not form branches till P3. Collateral branches start to form at P4 at the level of layer $\mathrm{V}$ neurons in the cortical plate and continue to increase at later stages (boxed region).

Since the lack of Sip1 affected axonal growth we wanted to test whether Sip1 deficiency also affects the formation of ipsilateral axonal collaterals. In order to investigate the normal course of collateral formation, we electroporated eGFP expressing plasmid at E15.5 in wild type embryos and harvested the embryos at subsequent postnatal stages (P0 to P7). In these experiments we could specifically visualize the UL neurons and their axonal branches in the deep layers of the cortex. The sections were also stained for Ctip2, which labels layer $\mathrm{V}$ neurons. We observed that between P0 and P3 as the UL neurons migrate and take up their final position in the cortical plate, they in parallel project axons to the white matter with no visible axonal collaterals. By $\mathrm{P} 4$, the first branches of ipsilateral axonal collaterals started to appear at the level of layer $\mathrm{V}$ neurons in the cortex, demarcated by Ctip2 positive cells 
(Figure 16). Between P4 and P7, there was a steady increase in the density of these axonal branches. Hence, ipsilateral branching of UL axons commences at P4 and is very prominent at P7 (Figure 16). These experiments also indicated that the axonal collaterals are most probably formed by the interstitial mode of branch formation, as the branches appear from the main axon shaft many days after intial axon extension.

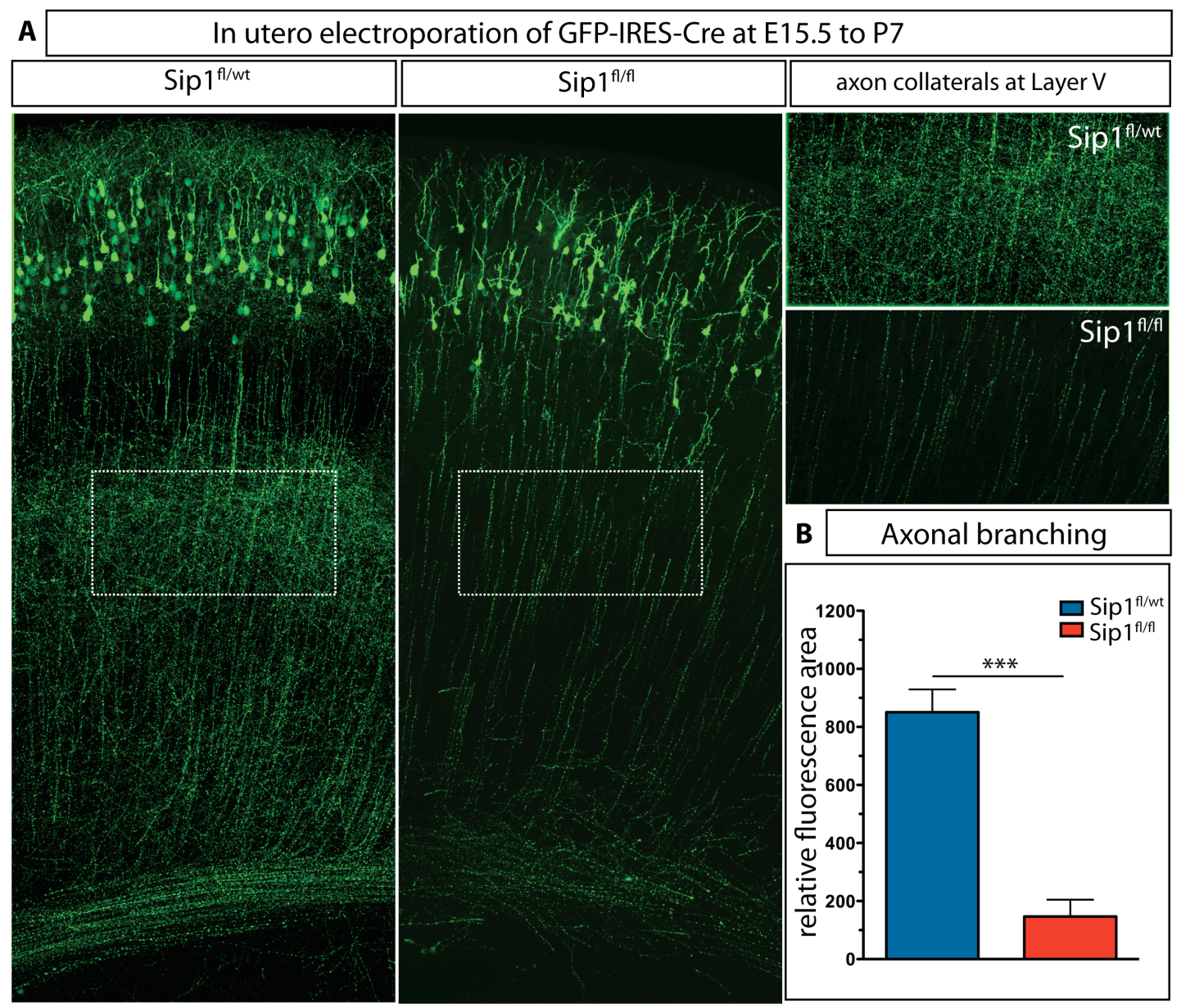

Figure 17. Sip1 deficient upper layer neurons do not form ipsilateral axon collaterals (A) In utero electroporation of GFP-IRES-Cre at E15.5 to P7 in Sip $1^{f / / w t}$ and Sip $1^{f / f l}$ embryos shows that Sip1 negative neurons do not form ipsilateral axon collaterals at the level of layer $\mathrm{V}$ neurons at P7, while the control brains show robust branching. (B) Quantification of the extent of branching at layer V between Sip1f/wt and Sip1f/fl sections confirms the absence of branching in the Sip1 deficient scenario. Relative fluorescence area in layer V: Sip $17 / w t=850.78$ $\pm 78.598, \operatorname{Sip} 1^{f / f l}=147.05 \pm 57.909$, $\mathrm{p}$ value $=0.0003$

To study the ipsilateral axon collateral formation in the presence and absence of Sip1, we electroporated GFP-IRES-Cre plasmid into Sip $1^{f l / f l}$ and Sip $1^{f / / w t}$ 
embryos at E15.5 and harvested the pups at P7, a stage when ipsilateral axonal collaterals are well established. We observed that while the neurons in the Sip $1^{f / w t}$ cortex projected axons that form a dense meshwork of branches at layer V, Sip1 lacking upper layer neurons in the Sip1fl/fl brains did not form any branches at all (Figure 17A). To quantify this effect we calculated the fluorescent area of the axonal collateral at layer $\mathrm{V}$ within a radial unit relative to the number of electroporated neurons. There was an approximate 6-fold decrease in relative fluorescence area at layer $\mathrm{V}$ in $\operatorname{Sip} 1 f l f l$ brains when compared to Sip1f/wt brains (Figure 17B). All values are depicted as mean \pm standard deviation, Sip $1^{f / / w t}=850.78 \pm 78.598$, Sip 1 fl/fl $=147.05 \pm 57.909, p$ value $=0.0003, \mathrm{n}=3$.

\subsection{Microtubule associated protein - ninein, is downregulated}

\section{in the Sip1 mutant}

Sip1 deficient neurons show defects in axonal extension and axonal branching. In order to identify Sip1 targets that control axonal growth and collateral formation we performed microarray experiments at E16.5 searching for genes down-regulated in the Sip1 deficient cortex. This analysis revealed that ninein, a centrosome and MT minus-end binding protein was downregulated in the absence of Sip1. We verified these findings by performing immunohistological staining with anti-ninein antibody on E16.5, Sip $1^{f / w t}$ Nex $x^{C r e}$ and Sip $1^{f / f l}$ Nex ${ }^{C r e}$ embryos. Within the Sip $1^{f l / w t}$ Nex $\mathrm{Cre}$ neocortex, ninein was strongly expressed in the cortical plate and the ventricular zone. Expression of ninein was also detected within the white matter. In the Sip 1 fl/fl Nex Cre mutant, a severe downregulation of ninein expression in the cortical plate as well as in the white matter of the cortex could be observed. The expression of ninein was unaltered in the progenitor zone (Figure 18). 


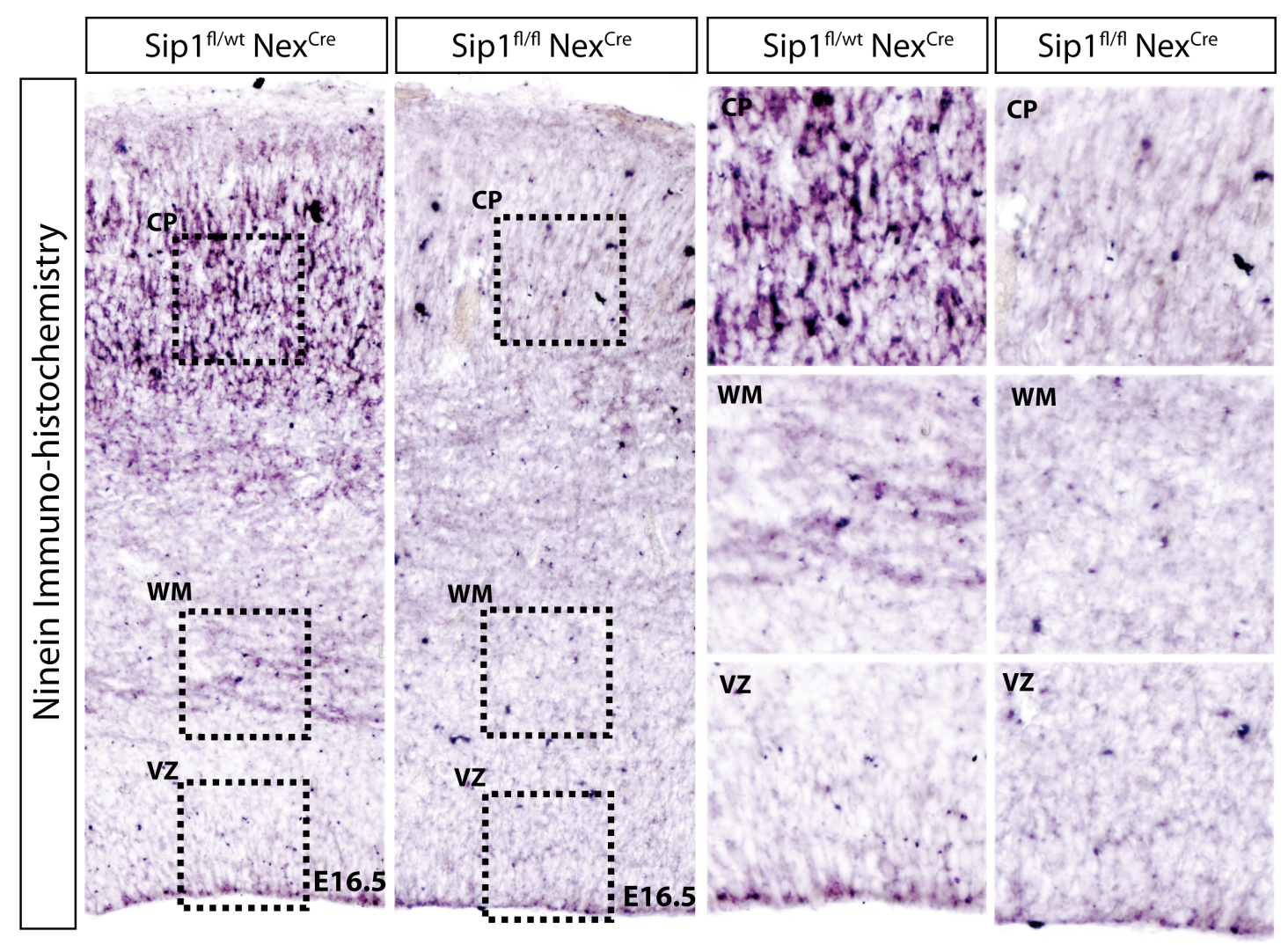

Figure 18. Ninein is downregulated in the Sip1 mutant cortex

Immunohistochemistry shows that ninein is expressed in the cortical plate $(\mathrm{CP})$, white matter (WM) and the ventricular zone (VZ) in the Sip1f//wt NexCre brains at E16.5, but is down regulated in the CP as well as WM of Sip $1^{f / f l} \mathrm{Nex}{ }^{\mathrm{Cre}}$ embryos. The expression of ninein at the VZ is unaffected in the Sip $1^{f / f l} \mathrm{Nex}$ Cre embryos.

\subsection{Sip1 regulates ninein expression through direct binding}

To test whether Sip1 directly binds to the ninein promoter region to regulate its transcription, we performed Chromatin immunoprecipitation (ChIP) assay on E16.5 wild type cortices. We used E16.5 Sip1 flfl Nex ${ }^{\mathrm{Cre}}$ mutant cortices as control. The immuno precipitated DNA was analyzed through quantitative real time PCR for 5 different regions within $10 \mathrm{~Kb}$ upstream of the ninein transcription start site (TSS), denoted as Nin-P1 to Nin-P5 that were found to have the bipartite Sip1 consensus binding motif: CACCT(G) (Figure 19A). The ChIP assay showed 7 to 10 -fold (Sip1:IgG) relative enrichment in all five regions tested. Sip1 flffl Nexcre mutant cortex showed no relative enrichment (Figure 19B). 


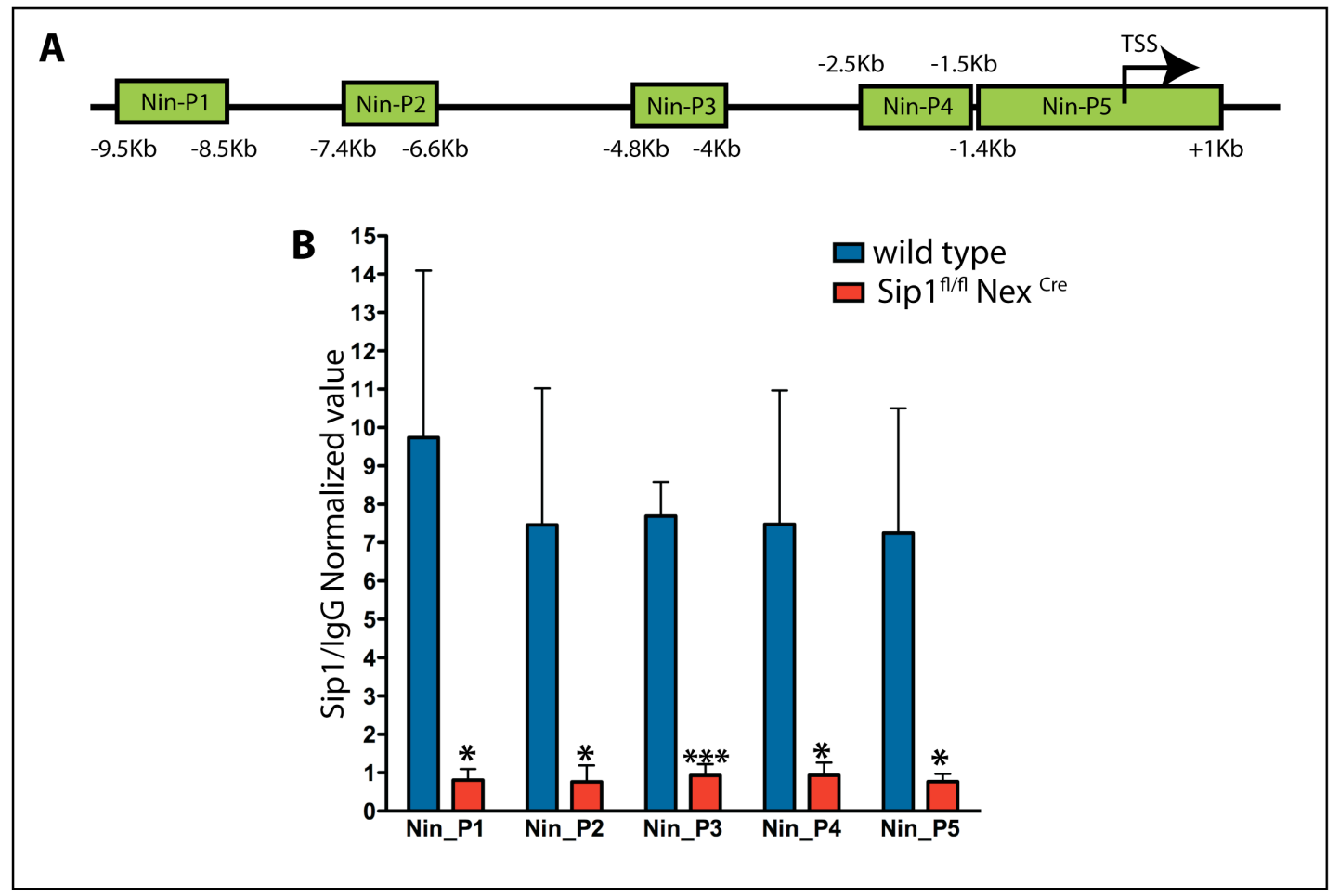

Figure 19. Sip1 directly binds to ninein promoter region and regulates its expression

(A) Five different regions with Sip1 consensus binding sequence within $10 \mathrm{~Kb}$ upstream region of ninein transcription start site were selected; Nin-P1 to Nin-P5. Primers to amplify 150-200 bp regions within the selected regions were designated for the ChIP assay. (B) ChIP data shows significant enrichment in all five regions tested in wild type tissue when compared to Sip1 mutant tissue. Wild type, $n=4$ (where each sample (n) contained pooled cortices from two brains): Nin-P1 $=9.7335 \pm 4.3579$, Nin-P2 $=7.4594 \pm 3.5617$, Nin-P3 $=7.6909$ \pm 0.8916, Nin-P4 $=7.4790 \pm 3.4918$, Nin-P5 = 7.2495 $\pm 3.2511 ;$ Sip1 $1_{\text {flfl }}$ Nex Cre, $n=3$ (where each sample (n) contained pooled cortices from two brains): Nin-P1 $=0.8060 \pm 0.2880$, Nin-P2 $=$ $0.76255 \pm 0.4252$, Nin-P3 $=0.9289 \pm 0.2925$, Nin-P4 $=0.9332 \pm 0.3278$, Nin-P5 $=0.7662 \pm 0.2036$; $p$-values Nin-P1 $=0.0259$, Nin-P2 $=0.0316$, Nin-P3 $=0.0001$, Nin-P4 $=0.0323$, Nin-P5 $=0.0278$.

All values are represented as mean of ratio of Sip1 normalized to IgG values \pm standard deviation. Wild type, $n=4$ (where each sample (n) contained pooled cortices from two brains): Nin-P1 $=9.7335 \pm 4.3579$, Nin-P2 $=7.4594 \pm 3.5617$, Nin-P3 = 7.6909 \pm 0.8916, Nin-P4 $=7.4790 \pm 3.4918$, Nin-P5 $=7.2495 \pm 3.2511$; Sip $1^{f / f l}$ Nex Cre, $\mathrm{n}=3$ (where each sample (n) contained pooled cortices from two brains): Nin-P1 $=0.8060 \pm 0.2880, \mathrm{Nin}-P 2=0.76255 \pm 0.4252, \mathrm{Nin}-\mathrm{P} 3=0.9289 \pm$ 0.2925, Nin-P4 $=0.9332 \pm 0.3278$, Nin-P5 $=0.7662 \pm 0.2036$; $p$-values Nin-P1 $=$ 0.0259, Nin-P2 $=0.0316$, Nin-P3 $=0.0001$, Nin-P4 $=0.0323$, Nin-P5 $=0.0278$. 


\subsection{Sip1 binding to the ninein enhancer is functional}

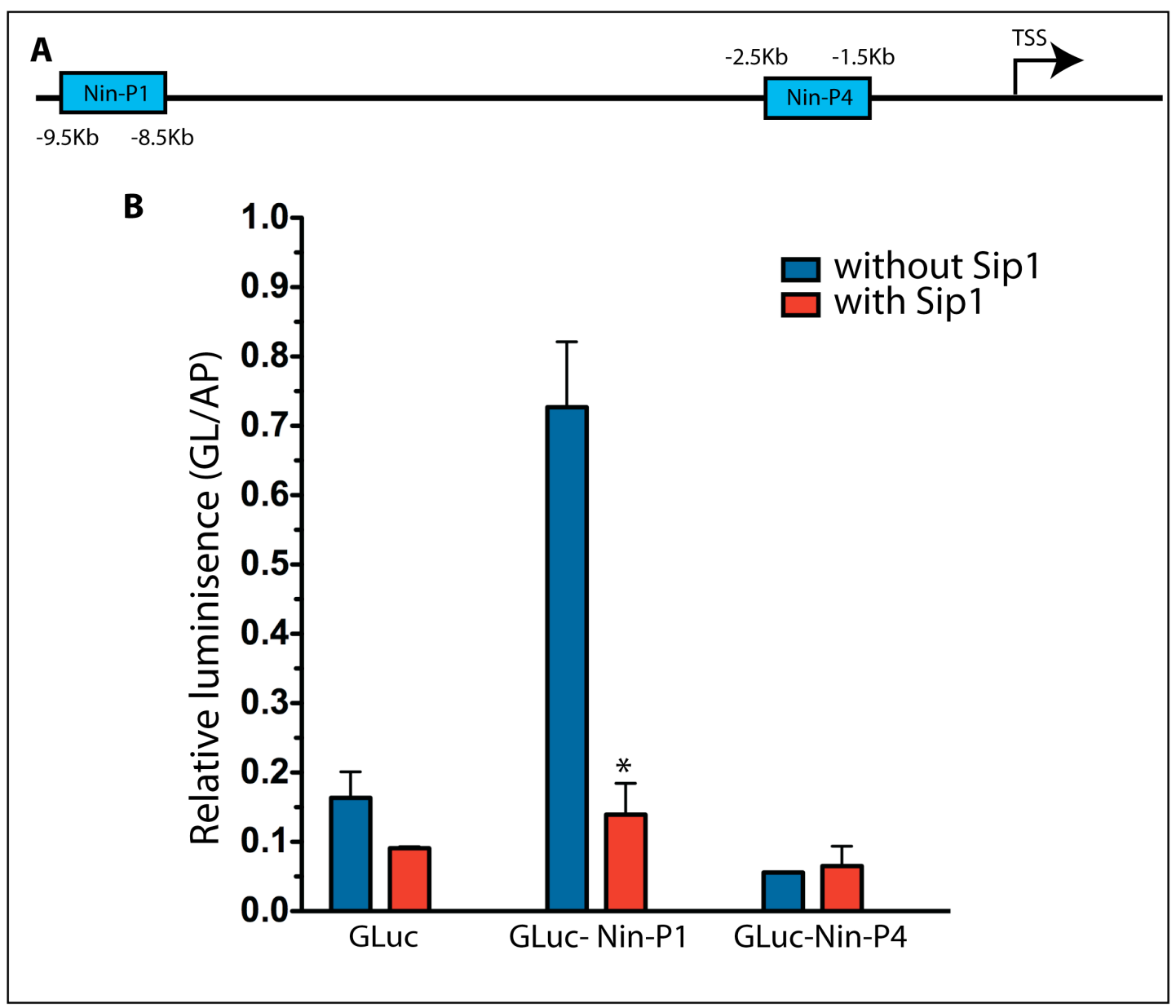

Figure 20. Luciferase assay confirms functional binding of Sip1 to ninein upstream region

(A) Two of the five promoter elements upstream of ninein coding region tested in the ChIP assay were selected and cloned into a plasmid upstream of Gaussia luciferase called, GLucNin-P1 and GLuc-Nin-P2. (B) There is a significant decrease in relative luminescence on Sip1 binding to Nin-P1 but not to Nin-P4. GLuc $=1.6353 \pm 0.037$, GLuc + Sip1 $=0.0908 \pm 0.002, p-$ value = 0.078; GLuc-Nin-P1 = $0.7269 \pm 0.0944$, GLuc-Nin-P1 + Sip1 $=0.1393 \pm 0.0450, p-$ value $=$ 0.0437; GLuc-Nin-P4 = $0.055 \pm 0.0005$, GLuc-Nin-P4 + Sip $1=0.0651 \pm 0.0285 ; p-$ value $=0.6259$.

To test the functionality of the binding of Sip1 to the ninein enhancer regions, we performed an in vitro luciferase assay. Two of the five different promoter regions upstream of the ninein transcription start site that were tested for the ChIP assay were chosen, namely Nin-P1 and Nin-P4 (Figure 20A). They were cloned upstream of Gaussia luciferase and expressed in HEK293T cells either with or without Sip1. Luminescence from the Gaussia luciferase was measured relative to the luminescence from alkaline phosphatase used as a control. We observed a significant decrease in transcriptional activity in Nin- 
P1 enhancer by 5.2 fold when Sip1 was co-transfected. Nin-P4 as well as the control plasmid did not show a change in relative luminescence (Figure 20B). In vivo, ninein expression is downregulated with the loss of Sip1 (Figure 18), thus attributing the role of a transcriptional activator to Sip1, whereas in the heterologous system used for the luciferase assay, Sip1 behaves as a transcriptional repressor. This maybe due to the absence of essential cofactors in the heterologous system used, which might be required for Sip1 to behave as a transcriptional activator. All values have been indicated as relative luminescence ratio between Gaussia luciferase and alkaline phosphatase \pm standard deviation, averaged over three independent experiments; G-Luc = 0.1635 $\pm 0.0376, G-L u c+S i p 1=0.0908 \pm 0.0022 ;$ GLuc-Nin-P1 $=0.7269 \pm 0.0944$, GLuc-Nin-P1 + Sip1 $=0.139 \pm 0.0450 ;$ GLuc-Nin-P4 $=0.0557 \pm 0.0005$, GLuc-Nin$P 4+\operatorname{Sip} 1=0.0651 \pm 0.0285$. Comparing with and without Sip1 co-transfection: GLuc-NinP1 p-value=0.0437, GLuc-Nin-P4 p-value =0.6259, $\mathrm{n}=3$.

\subsection{Ninein can be detected in the axons, axonal branches and}

\section{the axonal growth cone of developing neurons}

Ninein has been shown to bind to the minus-end of both centrosomal and non-centrosomal microtubules (Mogensen et al., 2000). It has been previously shown that in cultured neurons, ninein is present in the somato-dendritic compartment (Ohama \& Hayashi, 2009). Immunohistochemistry with an antininein antibody in E16.5 wild type cortex showed that apart from the cortical plate, ninein expression was also present in the cortical white matter (Figure 18). Hence, in order to verify if ninein is localized within the axon and the axonal growth cone of neurons, we transfected eGFP tagged ninein in DIV 1 cultured cortical neurons and analyzed them at DIV 2 and DIV 7. We cotransfected a dsRed expressing plasmid to be able to visualize the entire cellular morphology. At DIV 2 GFP-ninein puncta could be seen in the cell soma, in the axon and also within the growth cone. As expected no GFP could be seen within the nucleus (Figure 21). Analyzing the neurons at DIV 7 also showed similar localization. GFP-ninein puncta were visible in the dendrites, 
dendritic branches, axon, axonal branches and in the growth cone (Figure 22). It can hence be hypothesized that ninein might play an important role in modulating the cytoskeletal architecture and dynamics of these different cellular compartments.

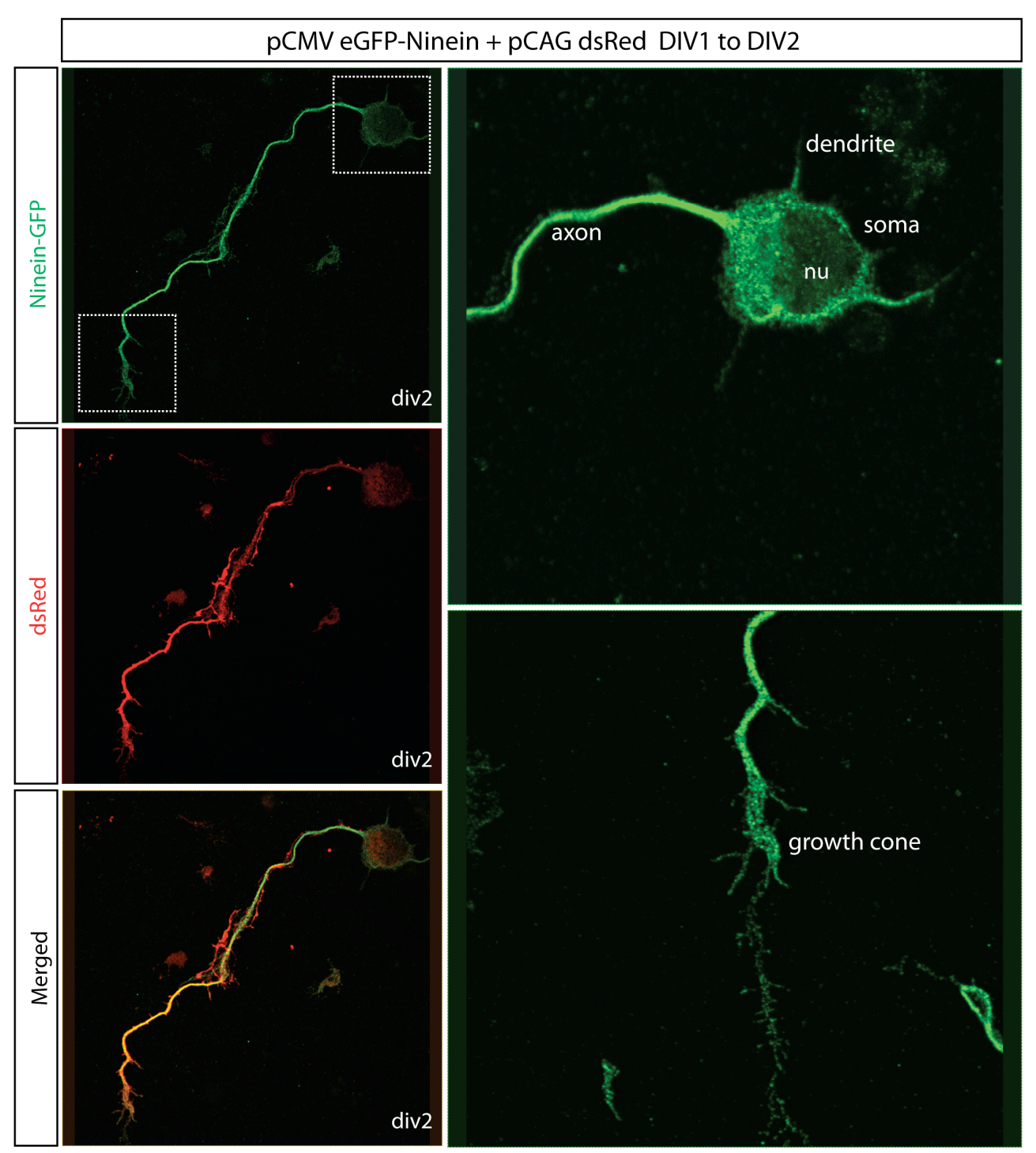

Figure 21. Ninein localizes to the soma, dendrites, axon and axonal growth cone

DIV 2 cortical dissociated neurons transfected at DIV 1 with eGFP tagged ninein (to help localize ninein) and pCAG-dsRed (to visualize the complete neuronal morphology) shows that GFP-ninein puncta are localized to the soma, dendrites, axon and the axonal growth cone. No GFP-ninein puncta are visible within the nucleus (nu). The higher magnification images correspond to the boxed regions. 


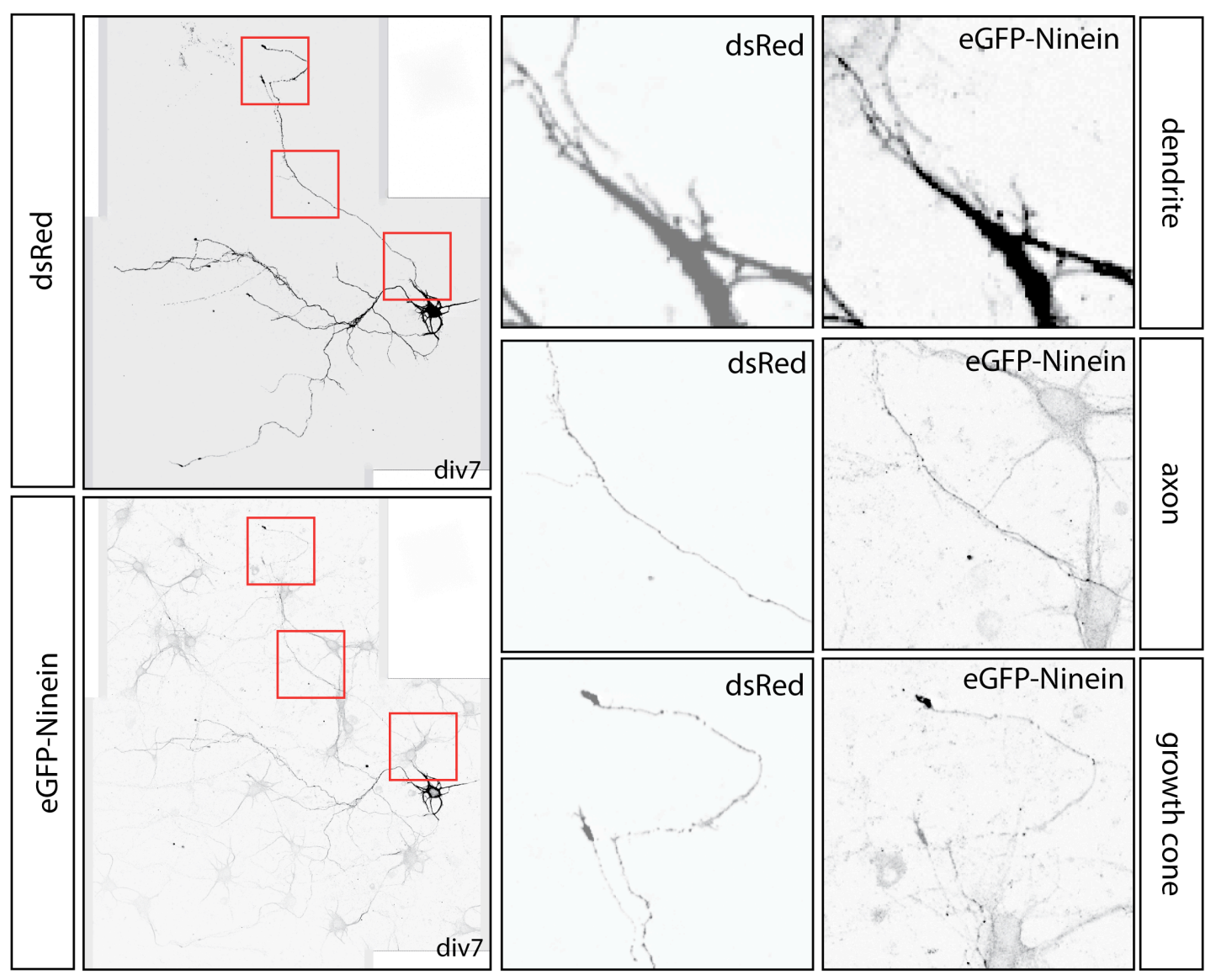

Figure 22. Ninein localizes to axonal and dendritic branches in neurons

DIV 7 cortical dissociated neurons transfected at DIV 1 with eGFP tagged ninein (to help localize ninein) and pCAG-dsRed (to visualize the complete neuronal morphology) reveal that GFP- ninein puncta also localize to axonal and dendritic branches that develop as the neurons mature, indicating that GFP-ninein is present in all cellular compartment of cortical neurons. Higher magnification images correspond to the boxed regions. 


\subsection{Axonal growth is retarded in Sip1 deficient neurons in vitro}

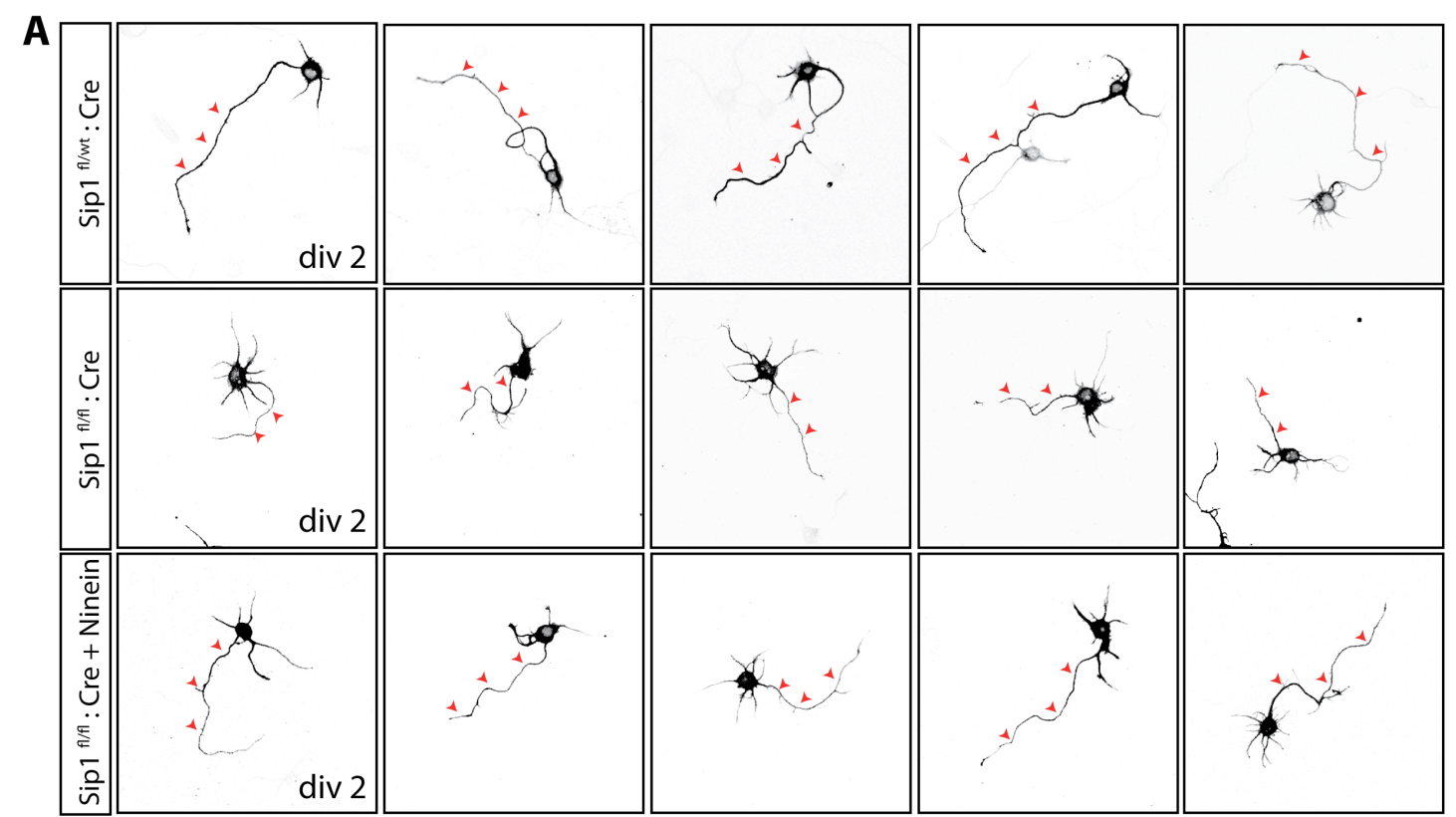

B
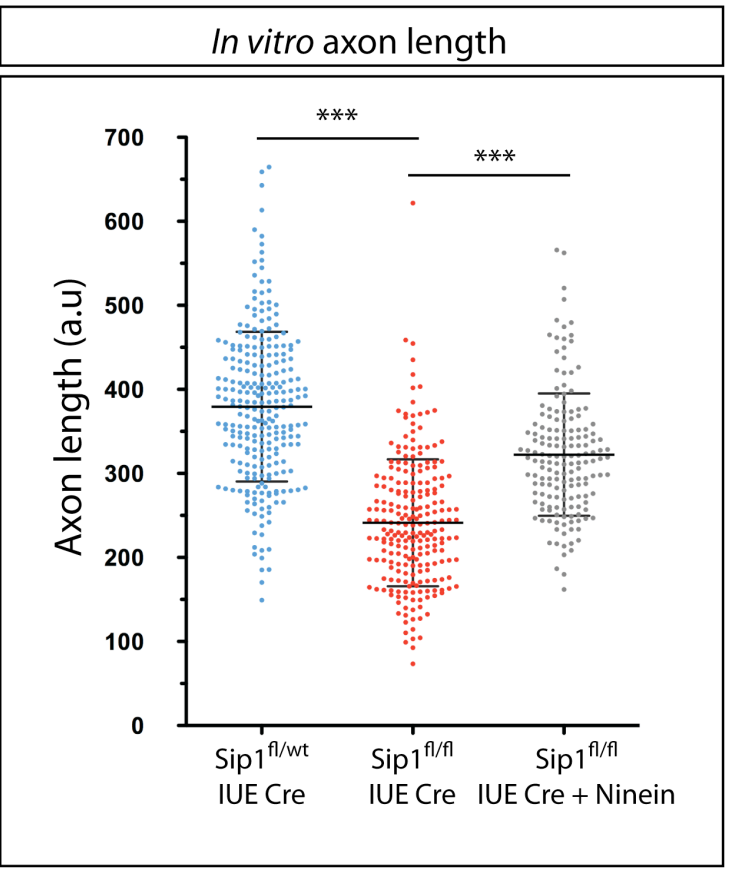

Figure 23. Sip1 negative neurons show stunted axonal growth in vitro, which is rescued by restoring ninein expression

(A) GFP-IRES-Cre expressing Sip1 $1 / f / f$ and Sip $1 f / / w t$ cortical dissociated neurons were cultured in vitro. (B) GFP-IRES-Cre expressing Sip1f/fll cortical dissociated neurons at DIV 2 had shorter axons when compared to Sip1f//wt neurons. (A, B) The axon length was rescued by co expression of $p C A G-F M F e G F P$ - ninein together with GFP-IRES-Cre in Sip1ffll cortical dissociated neurons. Sip1 $1^{f / w t}+\operatorname{Cre}(\mathrm{n}=258)=379.43 \pm 89.09$, Sip1f/fl $+\mathrm{Cre}(\mathrm{n}=233)=241.36 \pm$ $75.51, \operatorname{Sip} 1^{f / f l}+$ Cre + ninein $(\mathrm{n}=166)=322.41 \pm 72.82 ;$ p-values $<0.001$. 
We wanted to test if ninein, being a MT binding protein, that localizes to the axon and is down regulated in the Sip1 mutant, could influence axonal length. First we tested if Sip1 deletion from neocortical neurons would affect axonal length in vitro. Sip $1^{f / / w t}$ and Sip $1^{f / f l}$ embryos were electorporated with GFPIRES-Cre plasmid at E14.5 and brains were isolated at E17.5. The electroporated region of the cortex was isolated, dissociated and neurons cultured for 2 days in vitro and fixed. In utero electroporation was used instead of plasmid transfection in order to delete Sip1 as early as possible. The length of the axons of GFP positive neurons was measured. We observed that the average length of the axon of Sip1 deficient neurons was significantly shorter when compared to wild type neurons, thus reconfirming the cell autonomous requirement of Sip1 for axon extension (Figure 23A, B). To address whether ninein acts downstream of Sip1 to control axonal length, we performed similar experiments where ninein expression was restored in Sip1 deficient neurons. Expressing ninein in Sip1 deficient cells significantly increased the average length of the axon when compared to Sip1 negative neurons, although it was not restored to wild type levels (Figure 23A, B). All values depicted as average length \pm standard deviation. Sip1fl/wt + Cre $(\mathrm{n}=258)$ $=379.43 \pm 89.09, \operatorname{Sip} 1^{f / f l}+\operatorname{Cre}(\mathrm{n}=233)=241.36 \pm 75.51, \operatorname{Sip} 1^{f / f l}+$ Cre + ninein $(n=166)=322.41 \pm 72.82 ;$-values $<0.001$.

\subsection{Restoration of ninein expression in Sip1 deficient neurons rescues the corpus callosum}

Since overexpression of ninein could increase the axonal length of Sip1 deficient neurons in vitro we wanted to test if ninein could also potentially influence axonal growth in vivo. We hence electroporated a plasmid expressing eGFP tagged ninein that is precede by a floxed mCherry-polyA cassette, designated as $p C A G-F M F-e G F P$ Ninein, together with the GFP-IRESCre plasmid into Sip1fl/fl embryos at E12.5. Together the plasmid cocktail ensured onset of ninein expression only in the Cre positive and hence Sip1 
negative cells. The brains were analyzed at E18.5 and compared with Sip $1^{f / f l}$ and $\operatorname{Sip} 1^{f / w t}$ brains electroporated with only GFP-IRES-Cre plasmid.

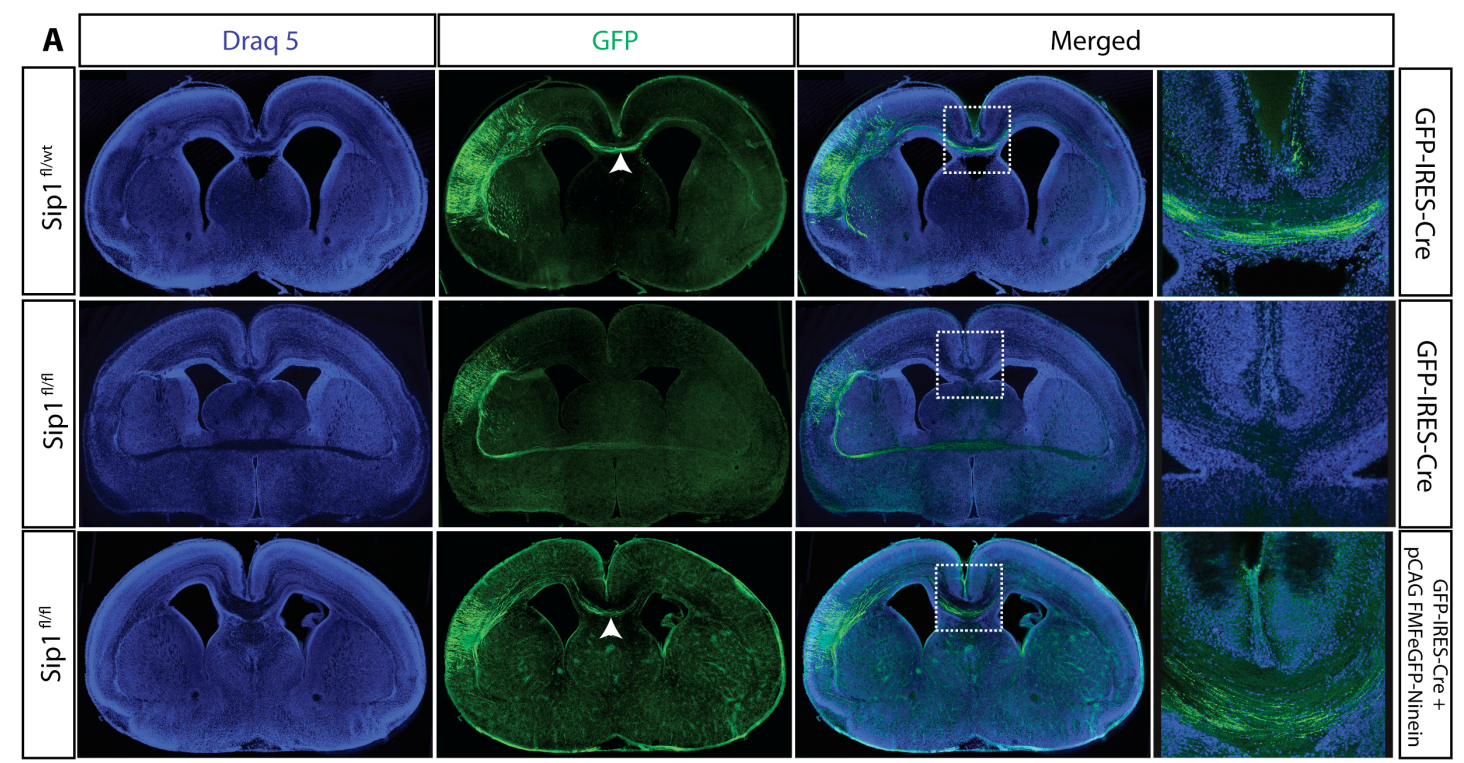

B Quantification of callosal rescue

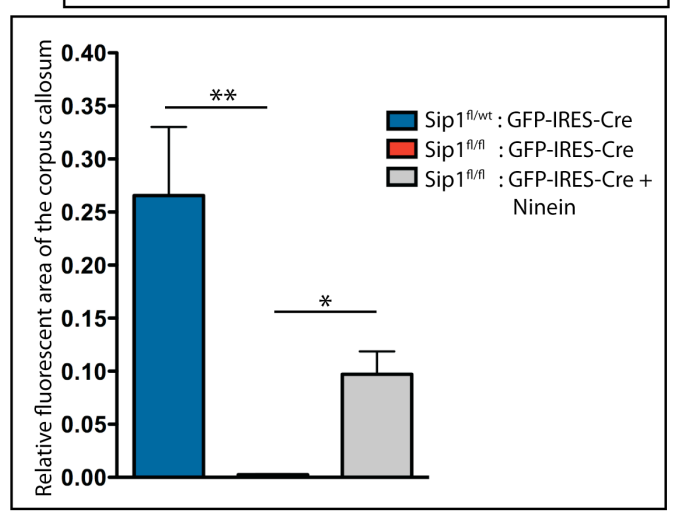

Figure 24. Restoring ninein expression partially rescues the corpus callosum in brains with cell autonomous deletion of Sip1

(A) $p$ CAG-FMF-eGFP Ninein co-expressed with GFP-IRES-Cre in Sip1flfll embryos at E12.5 and analyzed at E18.5. The corpus callosum is compared with that formed with GFP-IRES-Cre electroporation in Sip1fl/wt and Sip1fl/fl embryos (arrowheads). Sections are immunostained for GFP and Draq5. (B) Quantifying the thickness of the corpus callosum at the midline shows a partial rescue of callosal fibers in Sip1flfl embryos on co-electroporation of $p$ CAG-FMF-eGFP Ninein with GFP-IRES-Cre. Sip1fl/fl + Cre and ninein $=0.0972 \pm 0.0215$, when compared to Sip 1 fl/fl + Cre $=0.0025 \pm 0.0005$, $p$-value $=0.016$

We observed that while in Cre-IRES-GFP alone electroporated Sipt/fl embryos, the GFP positive axons did not form the CC, the neurons electroporated with Cre-IRES-GFP as well as ninein were able to extend their axons medially and cross the callosal midline into the contralateral hemisphere (Figure 24A). 
Quantifying the thickness of the CC at the midline showed that similar to the in vitro rescue of axonal length, the in vivo rescue of the CC was partial, as both the proportion of GFP positive axons crossing the midline and maximal axonal length at E18.5 were not restored to wild type levels (Figure 24B). The values are expressed as mean \pm standard deviation, $n=3$, Sip 1 fl/fl + Cre and ninein $=0.0972 \pm 0.0215$, when compared to Sip1flfl + Cre $=0.0025 \pm 0.0005, p$ value $=0.016$, Sip1fl/wt + Cre $=0.2656 \pm 0.06457$.

\subsection{Restoration of ninein expression in Sip1 deficient neurons restores ipsilateral axonal branching of layer II and III projection neurons}

We electroporated ninein together with Cre-IRES-GFP in Sip $1^{f l f l}$ embryos at E15.5 and harvested the brains at P7. Cre-IRES-GFP electroporation in Sip1 flffl embryos as well as Sip1f/wt embryos served as positive and negative controls. We observed that, on restoring ninein expression in Sip1 negative upper layer neurons, they were able to form ipsilateral axonal collaterals at the level of layer $\mathrm{V}$ neurons in the cortical plate (Figure 25A, B). The extent of arborization measured in terms of relative fluorescent intensity was comparable to the wild type (Figure 25B, C). Values expressed as mean \pm standard deviation, $\mathrm{n}=3$, Sip 1 flfl + Cre and ninein $=845.90 \pm 14.598$, when compared to Sip1flfll + Cre $=147.05 \pm 57.909$, p-value $=0.0011$, Sip1 $1^{f / w t}+$ Cre $=$ $850.78 \pm 78.598$. 


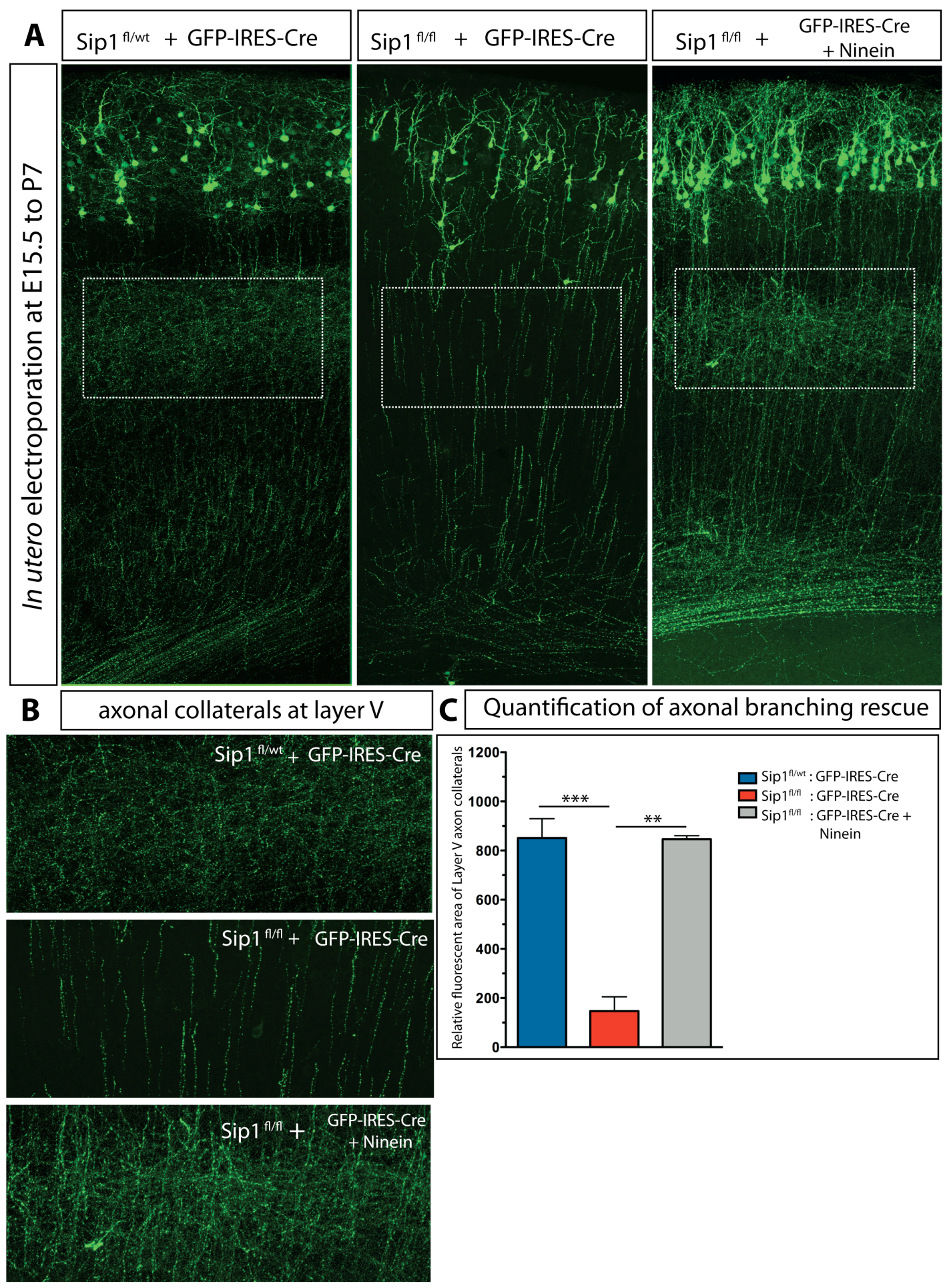

Figure 25. Axon collateral formation by upper layer neurons is restored by expression of ninein in Sip1 deficient neurons

(A) $p$ CAG-FMF-eGFP Ninein was co-expressed with GFP-IRES-Cre in Sip1/f/f embryos at E15.5 and analyzed at P7. The ipsilateral axonal collaterals formed at layer $\mathrm{V}$ in these brains was comparable with that formed with GFP-IRES-Cre electroporation in Sip1f/wt. GFP-IRES-Cre electroporation Sip $1^{f / f} / \mathrm{f}$ embryos shows no branching. (B) Higher magnification images of boxed area in panel (A). (C) Quantifying the axonal branching at layer $\mathrm{V}$ shows a rescue of collaterals in Sip1f/f/l embryos on co-electroporation of $p C A G-F M F-e G F P$ Ninein with GFPIRES-Cre. Sip1 $1_{f / f l}+$ Cre and ninein $=845.90 \pm 14.598$, when compared to Sip1fl/fl + Cre $=147.05 \pm$ 57.909, p-value $=0.0011$, Sip 1 ff/wt + Cre $=850.78 \pm 78.598$. 
3.25 Retarded microtubule growth contributes to the stunted growth of Sip1 deficient axons and is controlled by ninein

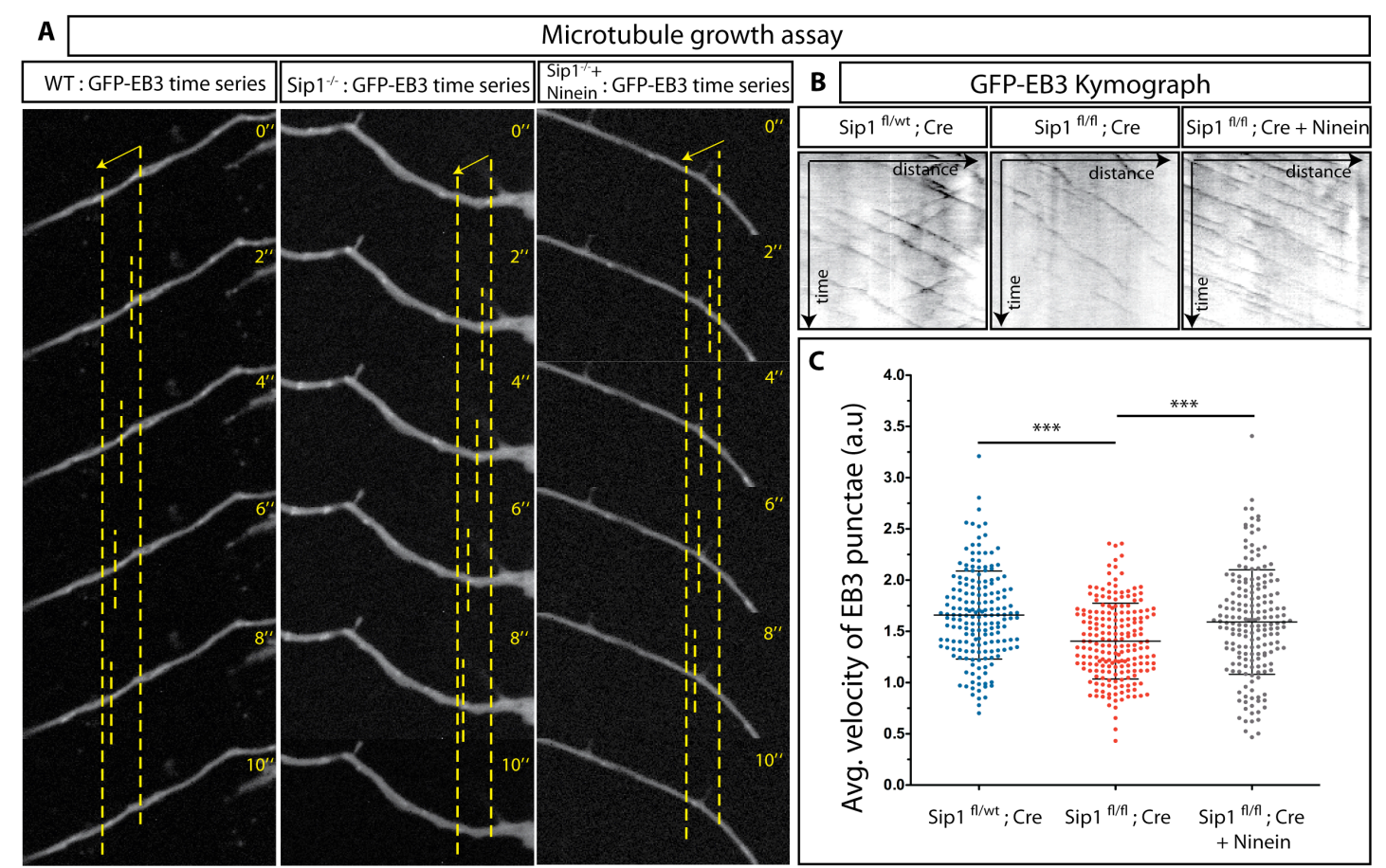

Figure 26. Microtubules grow slower in Sip1 deficient cells and the growth rate is restored in the presence of ninein

(A) Example of time series tracking the movement of GFP-EB3 +TIPs over 10 seconds in DIV 2, Sip $1^{f / w t}+$ Cre expressing, Sip $1^{f / f l}+$ Cre expressing and Sip $1 / f / f+$ ninein + Cre expressing cortical neurons. (B) Kymographs tracking the movement of GFP-EB3 comets over time, in all three experimental conditions. Slope of the graph indicates the speed of GFP-EB3 comets, which in turn correlates to the growth rate of microtubules. (C) Graph representing the average velocity of GFP-EB3 comets. Average velocity is represented as pixels traveled per second. Sip $1 / /$ wt + Cre expressing neurons $=1.65 \pm 0.42$, Sip $1^{f / f l}+$ Cre expressing neurons $=1.40$ \pm 0.369 , Sip $1 / f / f+$ ninein + Cre expressing neurons $=1.59 \pm 0.50, p$ values $<0.001$.

Since ninein is a MT binding protein that was able to rescue the axon length of Sip1 deficient neurons, we hypothesized that Sip1 through ninein may control the growth rate of axonal MTs. To assess this we used microtubule plus-end binding protein EB3 fused with GFP and tracked EB3-GFP comets through live imaging experiments (Stepanova et al., 2003). We firstly compared EB3GFP comet velocity in control neurons (Sip1 $1^{f / w t}$ neurons expressing Cre + EB3GFP) and in Sip1 deficient neurons (Sip fl/fl neurons expressing Cre + EB3-GFP) at DIV 2. The average anterograde velocity of EB3-GFP comets was significantly reduced in Sip1 deficient neurons probably contributing to the stunted axonal growth. When we examined axons of Sip1 deficient neurons 
where ninein expression was restored (Sip fl/f neurons expressing Cre + Ninein $+E B 3-G F P)$, we observed that the average anterograde velocity of EB3-GFP comets was significantly higher than in the axons of Sip1 deficient neurons and was almost restored to wild type levels (Figure 26). EB3-GFP comets from 13 to 16 neurons were analyzed per condition, only the comets that could be tracked for 10 seconds or more were included. Data is represented as average velocity \pm standard deviation: Controls $=1.65 \pm 0.42$, Sip 1 deficient neurons $=$ $1.40 \pm 0.369$, Sip1 deficient neurons + ninein $=1.59 \pm 0.50, p$ values $<0.001$.

\subsection{Ninein influences the stability of axonal microtubules}

We observed that re-expressing ninein in Sip1 deficient neurons could increase the rate of axonal extension as well as restore axonal branching in vivo. Further, we observed that ninein influences axonal growth by affecting MT growth. To understand whether ninein, being a MT binding protein also contributes to the stability of axonal microtubules, we performed a timed Nocodazole induced microtubule breakdown assay with cultured DIV 3, wild type cortical neurons expressing either eGFP or ninein $+e G F P$. The neurons were fixed and stained for acetylated tubulin (ac-tub) and GFP after 2 hrs, 4 hours, 6 hours and 8 hours of $5 \mathrm{mM}$ nocodazole exposure. We observed that at 2 hours both eGFP expressing control neurons and eGFP + ninein expressing neurons showed the presence of stable microtubules labeled by ac-tubulin along the length of the axon and other neurites of the cell (Figure 27A). By 4 and 6 hours of nocodazole exposure a higher percentage of control neurons showed the absence of ac-tubulin staining within the axon while eGFP + ninein expressing neurons seemed to be more resistant to microtubule break down (Figure 27C). Therefore, we observed that control neurons showed a steep decline in the proportion of cells with stable microtubules in the axonal compartments with increasing exposure time to nocodazole, falling to $22 \%$ at 8 hours. On the other hand ninein expressing cells were resistant to nocodazole breakdown and $85 \%$ of the cells contained stable microtubules within the axon at 8 hours (Figure 27B, C) 


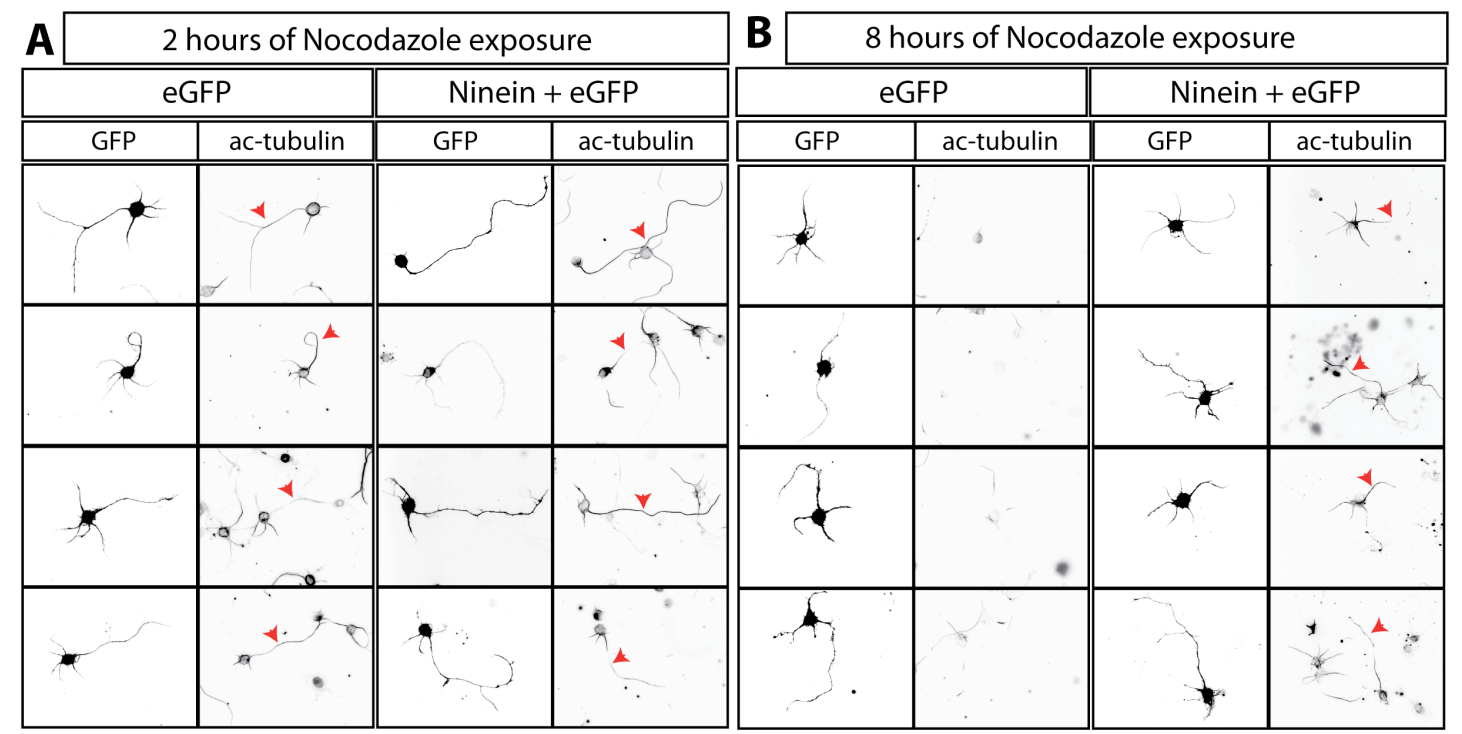

C Resistance towards nocodazole mediated

microtubule breakdown

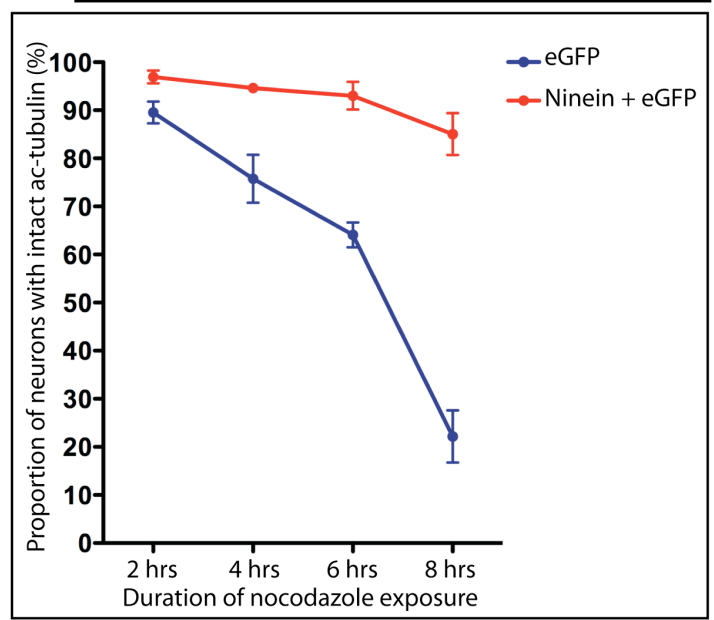

Figure 27. Ninein stabilizes microtubules in the axons

(A) Figure panel shown four example images of eGFP expressing DIV 3 cortical neurons as well as ninein $+e G F P$ expressing DIV 3 neurons after 2 hours of Nocodazole exposure, immunostained for GFP and acetylated tubulin. Ac-tubulin staining can be seen in the axons of both types of neurons (arrowheads). (B) Figure panel shown four example images of eGFP expressing DIV 3 neurons as well as ninein $+e$ GFP expressing DIV 3 neurons after 8 hours of Nocodazole exposure, immunostained for GFP and acetylated tubulin. Ac-tubulin staining can be seen in the axons of as ninein + eGFP expressing neurons but not in the eGFP alone expressing neurons (arrowheads). (C) Graph comparing the resistance of microtubule breakdown against increasing duration of Nocodazole exposure between eGFP expressing DIV 3 neurons as well as ninein $+e G F P$ expressing DIV 3 neurons. 2 hours: $e G F P=89.56 \% \pm$ 2.27, $e$ GFP + ninein $=96.92 \% \pm 1.35 ; 4$ hours: $e$ GFP $=75.77 \% \pm 5.00, e G F P+$ ninein $=94.29 \% \pm$ 0.76 ; 6 hours: $e G F P=64.09 \% \pm 2.60$, $e G F P+$ ninein $=93.02 \% \pm 2.88,8$ hours: $e G F P=22.17 \% \pm$ $5.41, e G F P+$ ninein $=85.04 \% \pm 4.35$. $p$-value $<0.001$ (two way ANOVA).

Hence the presence of ninein resists break down of microtubules induced by nocodazole suggesting that ninein acts as a microtubule-stabilizing molecule within the growing axon. The proportion of cells with stable microtubules 
was calculated by averaging the percentage obtained from three different cover slips for each condition and time point, 100 to 380 neurons were counted per cover slip. Data is represented as proportion of cells with stable microtubules \pm standard deviation. 2 hours: eGFP $=89.56 \% \pm 2.27$, eGFP + ninein $=96.92 \% \pm 1.35 ; 4$ hours: $\mathrm{eGFP}=75.77 \% \pm 5.00$, eGFP + ninein $=94.29 \%$ $\pm 0.76 ; 6$ hours: $\mathrm{eGFP}=64.09 \% \pm 2.60$, eGFP + ninein $=93.02 \% \pm 2.88,8$ hours: $\mathrm{eGFP}=22.17 \% \pm 5.41$, eGFP + ninein $=85.04 \% \pm 4.35$. $p$-value $<0.001$ (two way ANOVA).

Taken together these experiments describe the cell autonomous role of Sip1 in cortical callosal neurons, where Sip1 influences the rate of axonal growth as well as axonal collateral formation in vivo through its direct downstream effector ninein. Ninein, in turn exerts its influence on these process by regulating microtubule growth and stability. 


\section{PART III}

\section{Transcriptional control of Satb2 and Ctip2 over corpus callosum formation}

(Data presented in this part of the thesis are the results from the work performed along with Srinivas Parthasarathy, Charité-Berlin with equal contribution. Pilot experiments were performed by Paraskevi Sgourdou and have not been shown)

\section{$\underline{3.27 \text { Unc5C expression requires Satb2 and is restricted to callosal }}$}

\section{neurons.}

Satb2- mutants lack a corpus callosum suggesting the involvement of Satb2 in controlling, at least in part, the molecular program involved in CC formation (Alcamo et al., 2008; Britanova et al., 2008). In order to identify molecules down-stream of Satb2 that control CC formation we investigated changes in the expression of a variety of axon guidance molecules in the Satb2 $\%$ mutants. We assumed that such molecules should be downregulated in the Satb2 mutant cortex and that their expression should be confined to callosal neurons (Satb2 positive), while being excluded from corticofugal neurons (Ctip2 positive). Among others tested, the expression of the Netrin1 receptor - Unc5C satisfied these two criteria. Unc5C expression was previously shown to be down-regulated in Satb2 $/$ - mutants (Alcamo et al., 2008; Britanova et al., 2006; Srinivasan et al., 2012), where in situ hybridization against the Unc5C mRNA showed a complete loss of Unc5C from the Satb2\% cortical plate, with only expression in the subplate remaining (Figure 28A). Further, Unc5C has a very dynamic expression pattern during cortical development. At E14.5 Unc5C is predominantly expressed in the subplate. At E18.5 the expression of Unc5C in the cortical plate reaches a very high level and demonstrates a highlateral-low-medial gradient (Figure 28A, B).

To test whether ectopic and premature expression of Satb2 can induce Unc5C expression, we performed in utero electroporation of Satb2 and Ski expressing plasmids into the lateral ventricle at E12.5 and analyzed the brains at E14.5. Satb2 requires Ski as a co-factor for recruiting chromatin remodeling complexes and thus silencing it's targets (Baranek et al., 2012). 


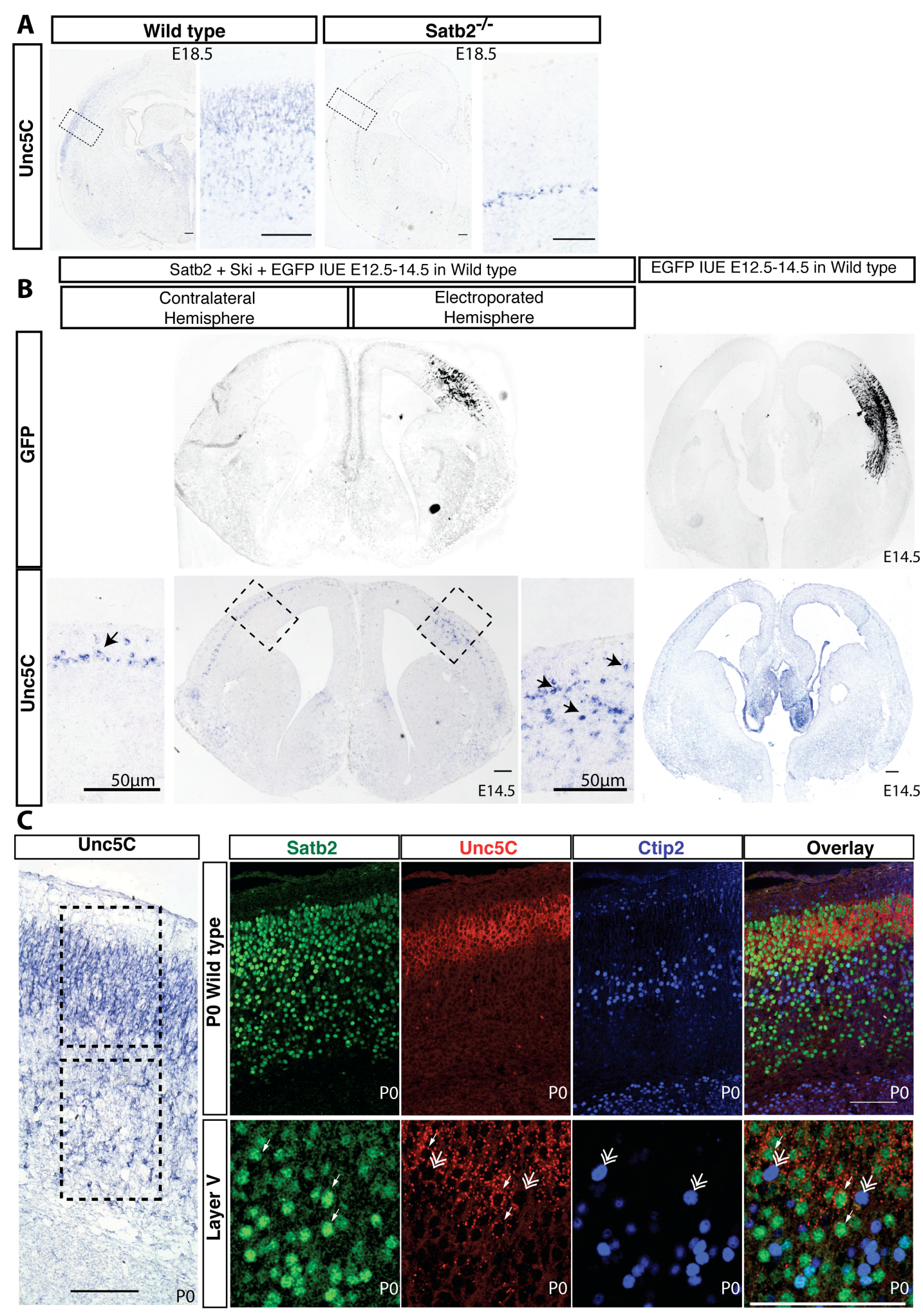

Figure 28. Unc5C is expressed by callosally projecting Satb2 positive neurons and Satb2 regulates Unc5C expression.

(A) Unc5C mRNA expression in wild type and Satb2- mutant mice at E18.5. In sections of Satb2- mutant brains, Unc5C continues to be expressed in only the subplate, but is absent in other cortical layers. (B) Unc5C expression in E14.5 wild type embryos in cells that were electroporated at E12.5 with Satb2/Ski/EGFP or EGFP control. GFP immunohistochemistry depicts the region electroporated. Unc5C in situ hybridization shows additional ectopic expression of Unc5C within cells electroporated with Satb2/Ski apart from the expression in 
the subplate seen in the contralateral hemisphere or in the control electroporation. (C) Unc5C mRNA expression with respect to Satb2 and Ctip2 expressing cells through immunofluorescence/fluorescence in situ hybridization in P0 wild type cortex. High magnification image of $\mathrm{P} 0$ wild type cortex shows that $U n c 5 \mathrm{C}$ is expressed primarily in the upper layers but also in a subset of cells in the deep layers - layer V (boxed regions) Unc5C mRNA expression coincides with the expression of Satb2 and is excluded from Ctip2 expressing cells. Layer V expression of Unc5C is restricted to cells expressing Satb2 (arrows) and absent in Ctip2 positive cells (double arrowheads). Scale bar $=100 \mu \mathrm{m}$, unless specified differently in the figure.

This experiment induced ectopic expression of Unc5C in the cells electroporated with Satb2 compared to a control construct (Figure 28B). To test our hypothesis that Unc5C is expressed by callosally projecting Satb2positive cells and absent in Ctip2-positive cells, we combined in situ hybridization (ISH) using a probe against Unc5C and immunohistochemistry (IHC) using antibodies against Satb2 and Ctip2. Unc5C mRNA is expressed predominantly in upper layers and weakly in deeper layers. Since combining ISH and IHC requires the omission of proteinase-K from the protocol, a physical limitation to the extent of probe penetration is an unavoidable drawback. Thus, even though in conventional ISH, Unc5C expression is seen in both upper and deeper layers, with FISH such a dual domain of expression was detected in separate experiments. We observed that Unc5C and Ctip2 were not expressed in the same cells in most cases whereas Unc5C and Satb2 were usually co-expressed. Even in layer V, Unc5C and Satb2-were coexpressed but neither was expressed in Ctip2-positive cells (Figure 28C).

The layer $\mathrm{V}$ expression of Unc5C specifically in cells negative for Ctip2 and positive for Satb2 indicated that Satb2 might also be able to repress Ctip2 in deep layer neurons, in addition to the previously reported repression in upper layer neurons (Britanova et al., 2008). In order to confirm this we counted the proportion of Ctip2 and Satb2 double positive cells within the deep layers of the cortex of wild type embryos and compared it with the proportion of Ctip2 and Cre double positive cells within the deep layers of the Satb2 mutant cortex (Figure 29A). Here Cre expression indicated the Satb2 deleted population of cells. We observed that there was a $70 \%$ increase in the 
number of Ctip2/Cre double positive cells within the deeper layers of the cortex in the Satb2 mutant (Figure 29B).
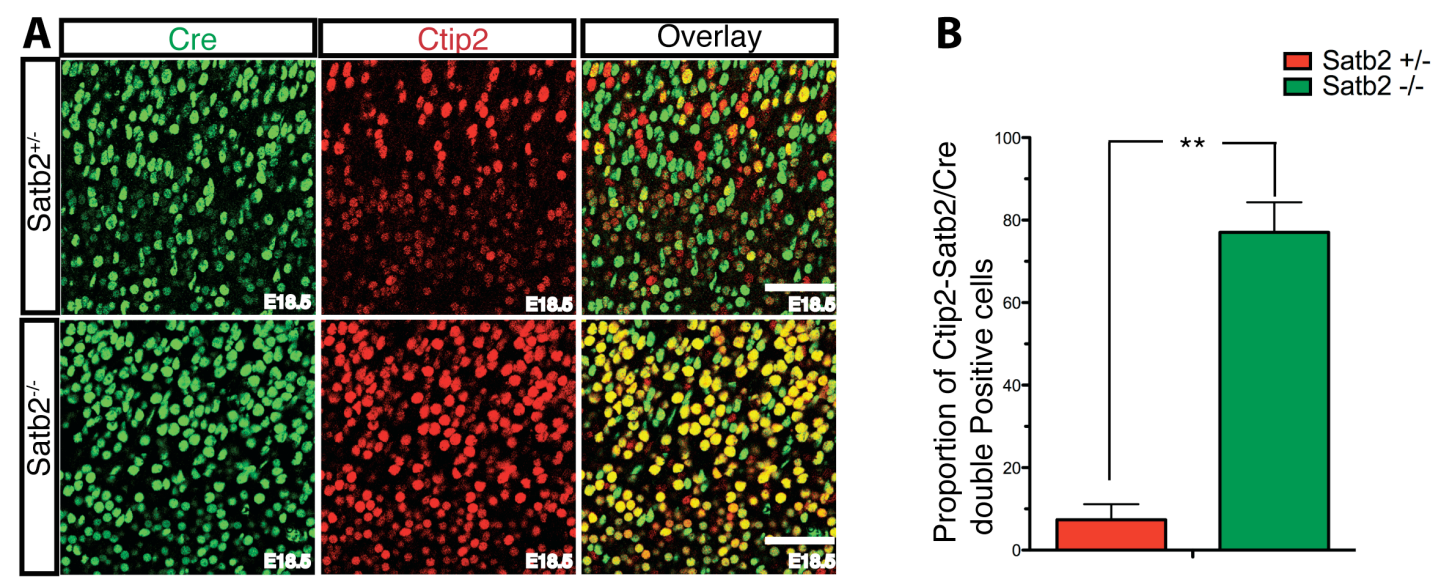

Figure 29. Ctip2 is up-regulated in the deep layers of the cortex upon Satb2 deletion

(A) Immunohistochemical staining using anti-Cre and anti-Ctip2 antibody was performed in Satb2+- and Satb2- brains. The proportion of deep layer Ctip2-positive cells that were also Satb2 (Cre) double positive in Satb2- brains as compared to Satb2+\% was plotted. (B) 8-fold increase was observed in the number of Ctip2-Cre double positive cells in Satb2\% mutant when compared to the heterozygote $(n=3, p$-value $=0.0006)$. Scale bar $=100 \mu \mathrm{m}$. Data are presented as average values \pm standard deviation (SD).

\subsection{Ctip2 directly represses Unc5C expression}

Since Unc5C and Ctip2 were not co-expressed, we hypothesized that Unc5C expression could be negatively regulated by Ctip2. To investigate this, we examined whether Unc5C expression would be restored in Satb2\%; Ctip2\% double mutants. We observed an increase of Unc5C expression in Satb2\%; Ctip2 $\%$ mutant cortex as compared to Satb2\% mutants. In Ctip2 single mutants we did not detect significant changes in Unc5C expression compared to controls (Figure 30).

This result indicated that in Satb2 mutants, the down-regulation of Unc5C could be the result of the ectopic upregulation of Ctip2. To test this hypothesis we over-expressed Ctip2 in the cortex. Ctip2 expression was validated in HEK293T cells and a confocal maximum Z-projection image for the electroporated region also confirmed the expression of Ctip2 in a large population of cortical cells (Figure 31A). An EGFP-expression plasmid was electroporated either alone or with the Ctip2 expression plasmid in wild type 
E12.5 embryos and the brains were harvested at E17.5. Subsequent sections were labeled with a probe against Unc5C mRNA. A decrease in Unc5C expression was observed in Ctip2 over-expressing cells within the electroporated cortical region, as compared to the contralateral hemisphere $(n=3)$ or EGFP-alone electroporated brains $(n=2$, Figure $31 B)$. In all further experiments wild type, Satb2 heterozygous, Ctip2 heterozygous and Satb2/Ctip2 compound heterozygous embryos have all been referred to interchangeably as controls as none of these genotypes show any deviation from the wild type phenotype for the pathway in question.

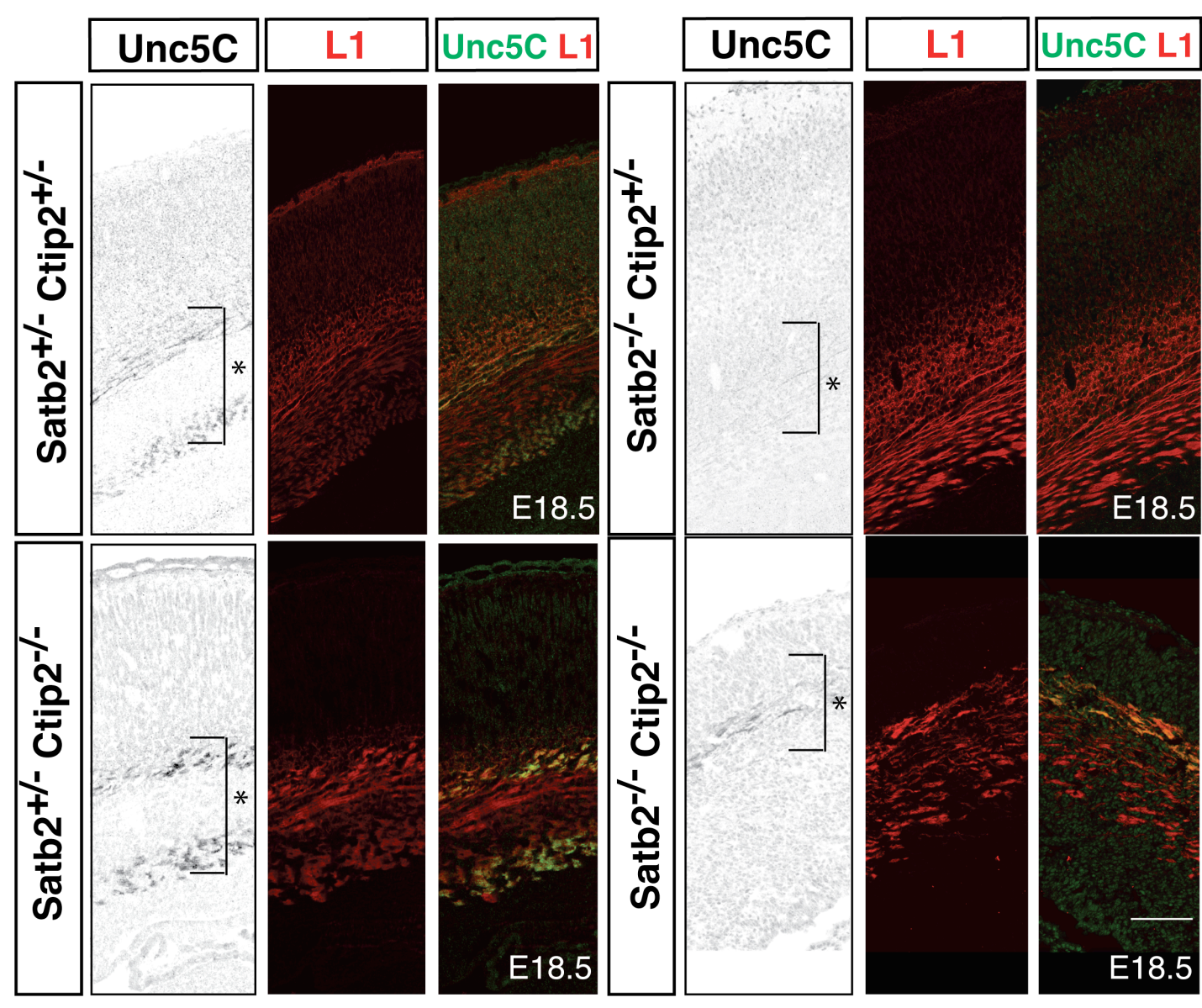

Figure 30. Unc5C expression is restored in Satb2 $-;$ Ctip2 $\%$ double mutants

Immunohistological staining against Unc5C and L1 in wild type, Satb2 $\%$, Ctip2 $2^{-}$and Satb2 $\%$ ;Ctip2 $\%$ double mutant brains. Unc5C expression is absent in Satb2\% brains when compared to wild type and Ctip2 $\%$ brains. The expression of Unc5C is restored in Satb2 $-;$ Ctip $2-$ double mutant brains. 

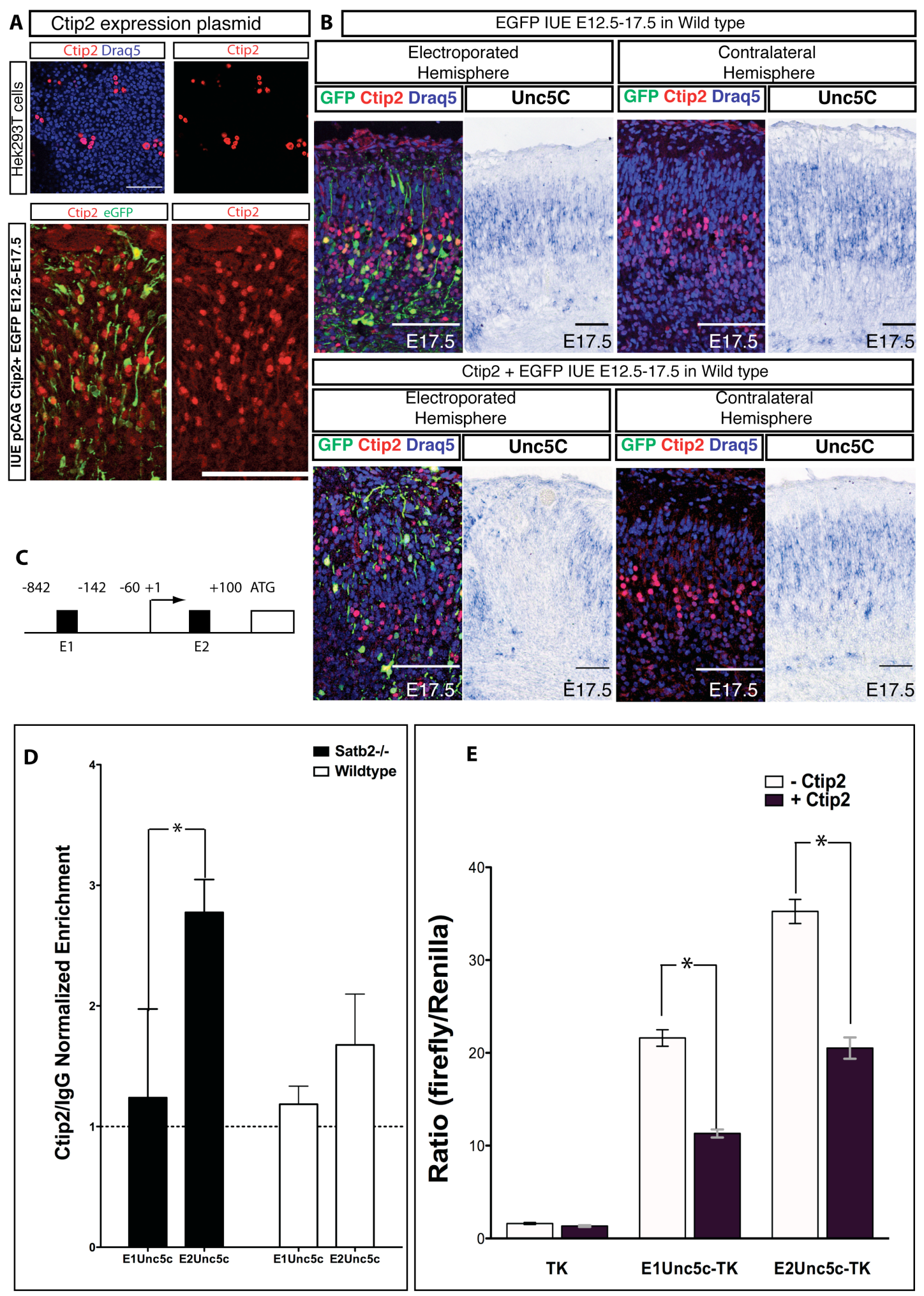

Figure 31. Ctip2 directly represses Unc5C expression.

(A)Plasmid expressing Ctip2 under the ubiquitous CAG promoter was transfected into HEK 293T cells. Immunohistochemistry of Ctip2 together with nuclear marker Draq5 confirmed the expression of Ctip2. Plasmid expressing Ctip2 expressed in cortical neurons by in utero electroporation at E12.5 and analyzed at E17.5. Immunohistochemistry of Ctip2 confirmed the expression of Ctip2 in vivo as well. Scale bar $=100 \mu \mathrm{m}$. (B) Unc5C expression in E17.5 wild type embryos in cells electroporated at E12.5 with Ctip2/EGFP or EGFP control within the electroporated as well as contralateral hemisphere. Subsequent section stained for GFP, Ctip2, 
Draq5 (a nuclear marker) immunohistochemistry. Unc5C mRNA expression is lost in the region electroporated with Ctip2 when compared to either the control electroporation or the corresponding region in the contralateral hemisphere. (C) Scheme showing the two regions in the Unc5C promoter where Ctip2 was predicted to bind. (D) Chromatin immunoprecipitation from P0 cortex of wild type and Satb2 $\%$ mutants show that Ctip2 binds to the Unc5C promoter region. $2.78 \pm 0.2722$ fold enrichment for E2 compared to $1.23 \pm 0.73$ for E1 in Satb2- $\mathrm{n}=3, p$ value $=0.027$ and $1.67 \pm 0.42$ fold enrichment for E2 compared to $1.18 \pm 0.14$ for E1 in wild type $\mathrm{n}=4$, n.s (E) Luciferase assay to demonstrate that Ctip2 binds to Unc5C genomic region and represses its expression. In the presence of full length Ctip2, 1.9 and 1.7 fold decrease in luminescence ratio was observed in case of E1 and E2 respectively. E1 and E2 being two putative Ctip2 binding sites. All $p$ values were $\leq 0.05$. Scale bar $=100 \mu \mathrm{m}$. The luciferase assay was performed by Olga Britanova, IBCh - Russian academy of Science, Russia

Next, we investigated whether Ctip2 directly represses Unc5C expression. An in silico search revealed two putative Ctip2 binding sites in the Unc5C promoter region, that we designated E1 and E2 (-842 to -142 and -60 to +100$)$ upstream of the transcription initiation site (Figure 31C). Chromatinimmunoprecipitation (ChIP) from wild type and Satb2-- cortices followed by qRT-PCR, showed that Ctip2 binds strongly to the E2 but not the E1 Unc5C promoter region. Since the wild type cortex consists of a relatively low Ctip2 population, Satb2- cortices were used as a means of enriching for Ctip2 positive cells in the cortex (Figure 31D, $2.78 \pm 0.2722$ fold enrichment for E2 compared to $1.23 \pm 0.73$ for E1 in Satb2-/-, n=3, p-value $=0.027$ (Student's t-test) and $1.67 \pm 0.42$ fold enrichment for E2 compared to $1.18 \pm 0.14$ for E1 in wild type, $n=4$, n.s).

To confirm this result we performed an in vitro luciferase assay. In these experiments the same two Unc5C upstream genomic regions E1 and E2 were inserted into a plasmid encoding the luciferase gene and transfected into cells with a plasmid encoding Ctip2. We observed a decrease in the activity of the Unc5C enhancer by approximately 1.9-fold and 1.7-fold, in the case of E1 and E2 respectively, when Ctip2 was co-transfected (Figure 31E, both p-values < 0.05). These results indicate that Ctip2 can directly repress Unc5C transcription. 


\subsection{Partial Restoration of the CC in Satb2 $\%$;}

\section{mutants}

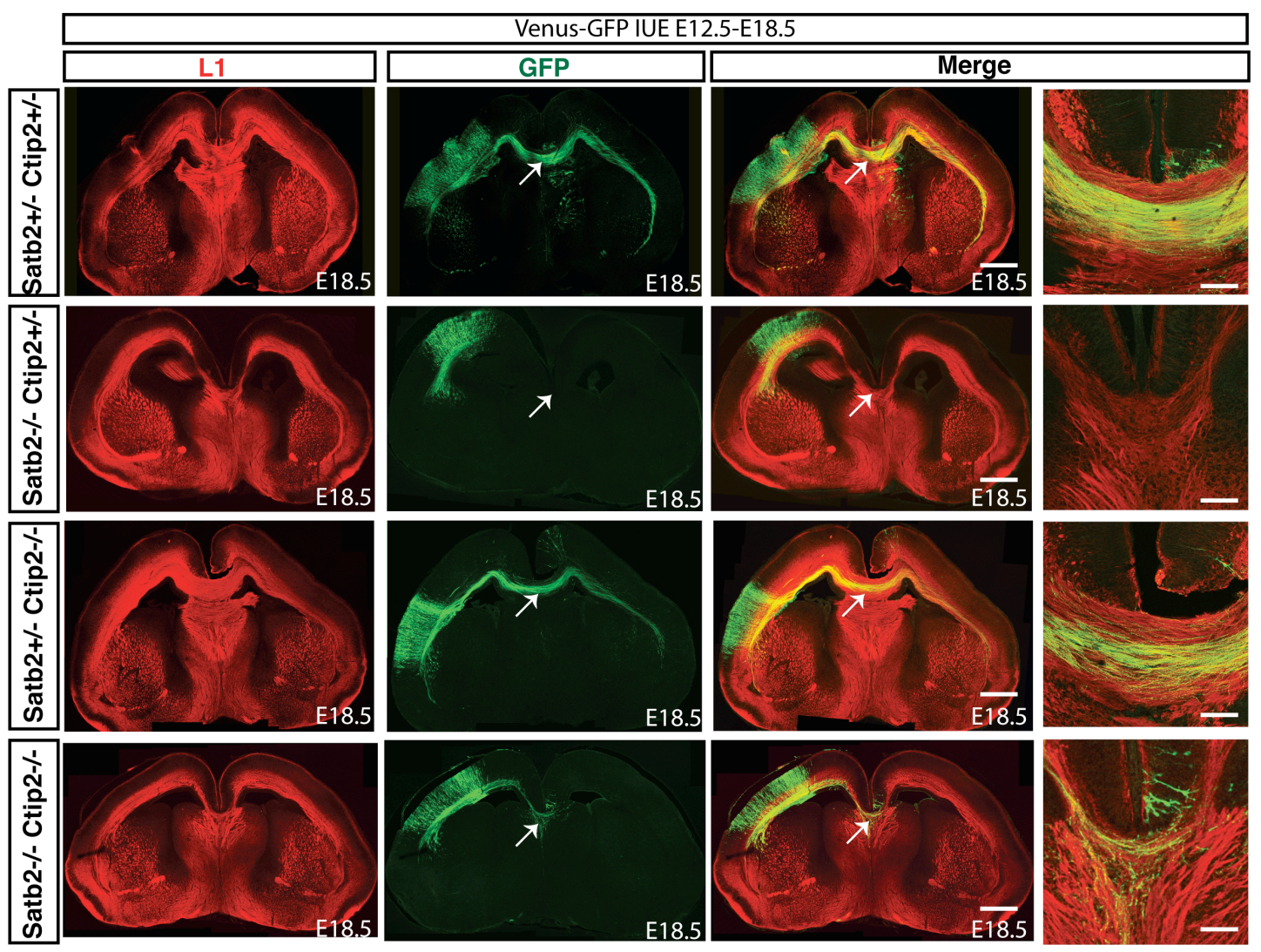

Figure 32. The corpus callosum is partially restored in Satb2-\%; Ctip2- compound mutants

The panel of images with higher magnification of the midline shows a series of experiments, wherein Venus GFP was electroporated into the developing cortex at E12.5 and the brains harvested and analyzed at E18.5. Electroporation of Venus GFP shows a normal CC in wild type and Ctip $2 \%$ embryos. Satb2 $\%$ mutant brains clearly demonstrate a complete absence of callosal fibers, wherein no fibers could be seen approaching the midline. Partial rescue of the CC could be seen in Satb2-\% Ctip2- compound mutants. Fibers manage to reach and cross the midline (arrows). The sections have been subjected to immunohistochemical staining for the neural cell adhesion molecule L1 and for GFP. Scale bar $=450 \mu \mathrm{m}$ for low magnification images and $100 \mu \mathrm{m}$ for high magnification images.

Others and we have previously shown that the deletion of Satb2 results in the ectopic up-regulation of Ctip2 and misprojection of callosal axons laterally (Alcamo et al., 2008; Britanova et al., 2008). Since Satb2 and Ctip2 regulate divergent genetic programs, that control the formation of interhemispheric versus corticofugal projections (Alcamo et al., 2008; Arlotta et al., 2005; Britanova et al., 2008), we investigated whether down-regulating Ctip2 in the Satb2 $\%$ cortex could restore CC formation. We analyzed Satb2- ; Ctip 2\% double mutant animals for the presence of the CC. To visualize callosal axons, we 
introduced a plasmid coding for Venus-GFP in neocortical cells by in utero electroporation at E12.5 and harvested the brains at E18.5. We found that while in Satb2 $\%$ animals, no cortical axons approached or even turned towards the dorsal midline, in Satb2- ;Ctip2- compound mutants, GFP positive axons approached, crossed the midline and invaded the contralateral cortex. It is noteworthy though that the number of crossing axons that projected into the contralateral hemisphere were fewer when compared to that seen in wild type and Ctip2 $\%$ littermates (Figure 32). The results were quantified by measuring the fluorescent area of the fibers projecting medially with respect to the fluorescent area of the electroporated region in the cortex (Figure 43).

\subsection{Satb2 deficient callosal neurons have defects in axon targeting and not outgrowth or midline guidance}

To rule out the possibility of axonal outgrowth defects in Satb2 negative neurons as a cause for the lack of extension of the axons contralaterally, we performed an in vitro axon-outgrowth assay using wild type and Satb2 mutant cortical explants, but did not observe an axonal outgrowth deficit in Satb2 deficient neurons (Figure 33A). Another possibility was that the axons were indirectly affected due to changes in the formation of midline guidepost structures in the Satb2 mutant and Satb2- $\%$ Ctip2 2 - double mutants (Niquille et al., 2009; Shu \& Richards, 2001). To examine this, we stained for midline guidepost cells: Sling neurons- with anti-calretinin (CR) and midline glia: with anti-GFAP. We did not detect any differences in the size or morphology of midline structures between the different genotypes suggesting that Satb2 deficient neurons are defective in axonal targeting and not outgrowth or midline crossing (Figure 33B). 

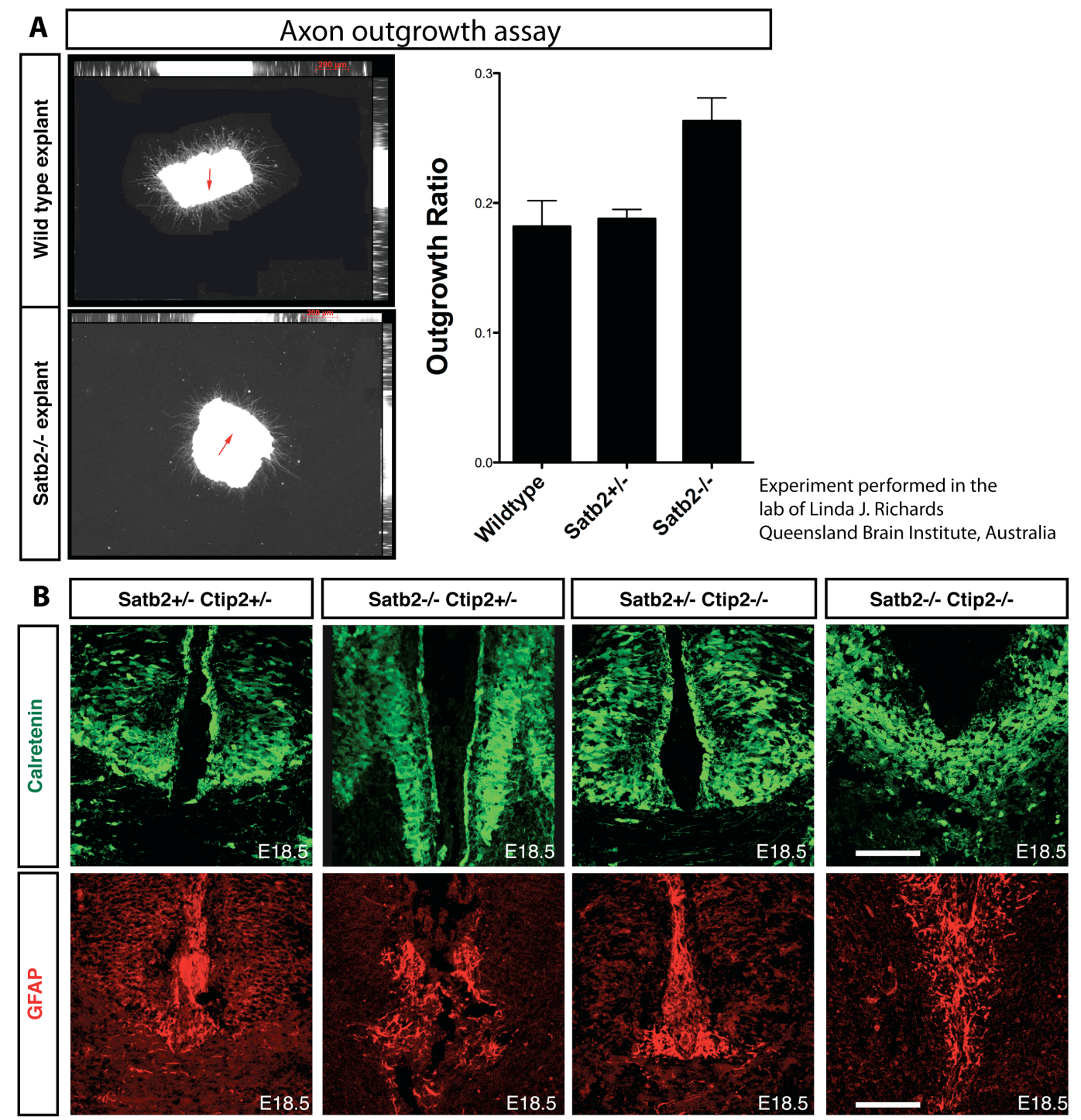

Figure 33. Satb2\% neurons do not show defects in neurite outgrowth and midline guidepost cells are not altered in Satb2 $\%$, Ctip $\%$ or Satb2 $\%$ Ctip2 $\%$ mutants

(A) Outgrowth ratio of axons from cortical explants of wild type, Satb2+/- and Satb2-- were compared. Satb2 deficient neurons did not show impairment in axonal growth, instead showed a slight increase in outgrowth ratio when compared to the controls ( $p$-value $=0.0037$ Student's t-test). (B) Midline guidepost cells were stained for using antibodies against Calretinin and GFAP in wild type, Satb2\%, Ctip2 $\%$ and Satb2 $\%$ Ctip2 $\%$ brains, showing comparable expression patterns and levels in all four genotypes. Scale bar $=100 \mu \mathrm{m}$.

\subsection{Restored expression of Unc5C in Satb2 ${ }^{-1}$ mutants results in}

\section{partial rescue of $\mathrm{CC}$}

Since the Satb2- - ; Ctip2 $\%$ double mutants showed a partial rescue of callosal crossing fibers and an increased Unc5C expression compared to single Satb2mutants, we tested whether we could rescue callosal fibers in Satb2\% mutants by over-expressing Unc5C. 


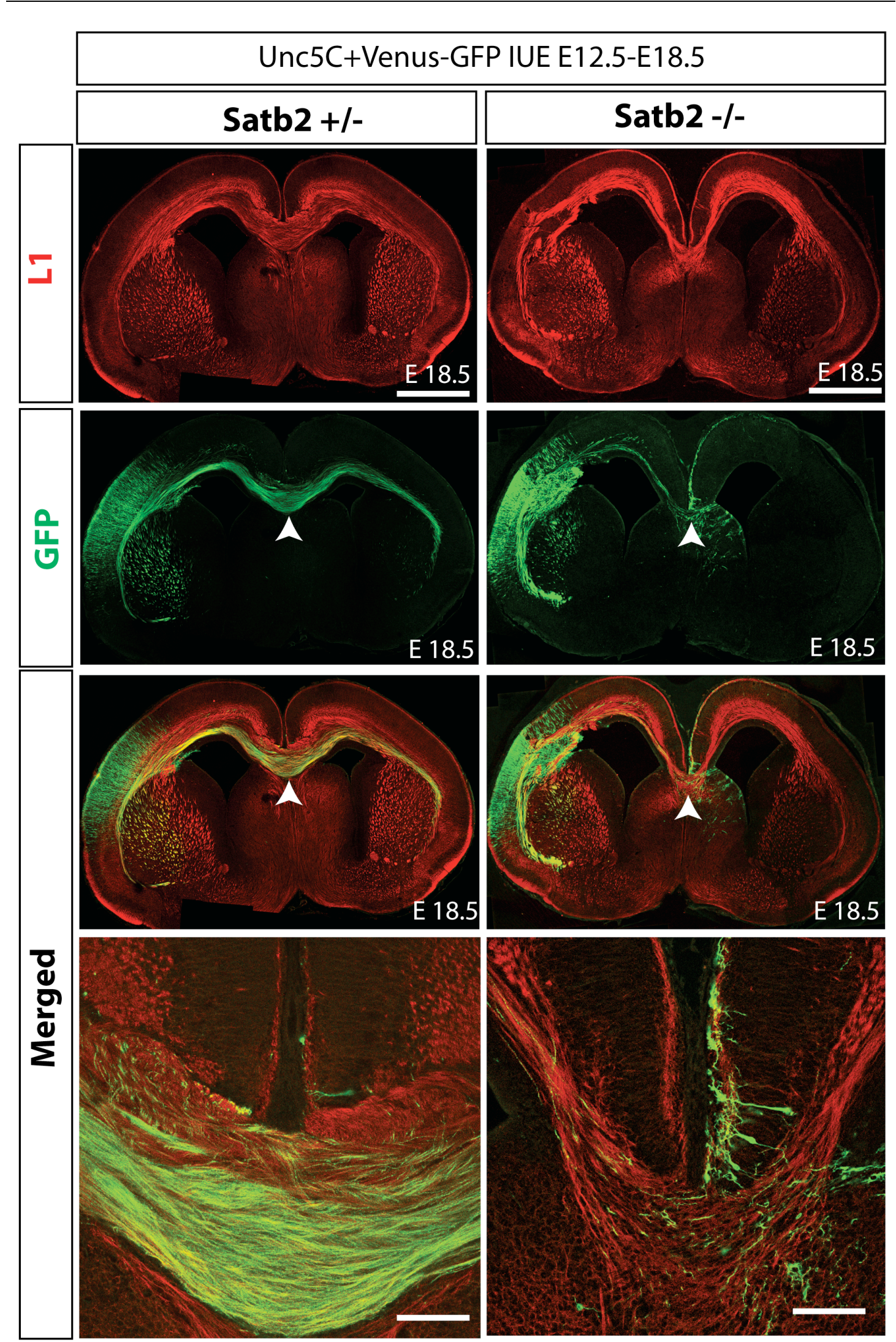

Figure 34. The corpus callosum is partially restored by over-expressing Unc5C in Satb2 mutant cortex.

Images show the result of Unc5C/EGFP over-expression in wild type and Satb2- mutant cortex. Over-expression of Unc5C in Satb2\% partially restores the CC where axons of electroporated neurons reach and cross the midline (arrow). Normally projecting callosal 
axons were observed in the control cortex. Scale bar $=450 \mu \mathrm{m}$ for low magnification images and $100 \mu \mathrm{m}$ for high magnification images.

An Unc5C expressing plasmid was co-expressed with Venus-GFP at E12.5 and compared with Venus-GFP expression alone in Satb2 $\%$ as well as control brains. We observed that axons of the Unc5C electroporated neurons in the Satb2 $\%$ cortex extended medially, and crossed the midline $(n=3)$. This rescue was again only partial as fewer axons crossed the midline compared to that in the control animals (Figure 34). Electroporation of only Venus-GFP in Satb2 $\%$ embryos served as a negative control where no callosally extending fibers were observed (Figure 32). The extent of callosal midline crossing with electroporation of Unc5C was comparable to the partial rescue obtained in Satb2- - ; Ctip2 - double mutants at E12.5 (Figure 32, 34, Quantification of CC rescue - Figure 43).

\subsection{Neocortical callosal axons are repelled by Netrin1.}

Unc5C has been shown to be a receptor for the secreted ligand Netrin1, mediating long range repulsive signaling (K. Hong et al., 1999; Serafini et al., 1996). Since we observed that the formation of callosal projections depended upon the presence of Unc5C expression, we tested whether these Unc5Cpositive callosally projecting neurons were also responsive to Netrin1. To test this we devised a slice culture system where wild type embryos were coelectroporated with either Unc5C and EGFP (Figure 35A) or only EGFP (Figure 35B) at E12.5 and harvested at E16.5. Slices prepared from these brains were cultured for 3 days in vitro and Netrin1 soaked agarose beads were placed in the midline at the position where callosal fibers cross. If the Unc5C positive callosal axons were responsive to the repulsive Netrin1 signal, then they would be prevented from crossing the midline upon encountering the ligand in their trajectory. When Unc5C positive fibers encountered Netrin1 soaked beads in the midline, they were repelled and did not cross the 
midline. Instead they misprojected ventrally or dorsally within the ipsilateral hemisphere showing an avoidance behavior towards the beads.

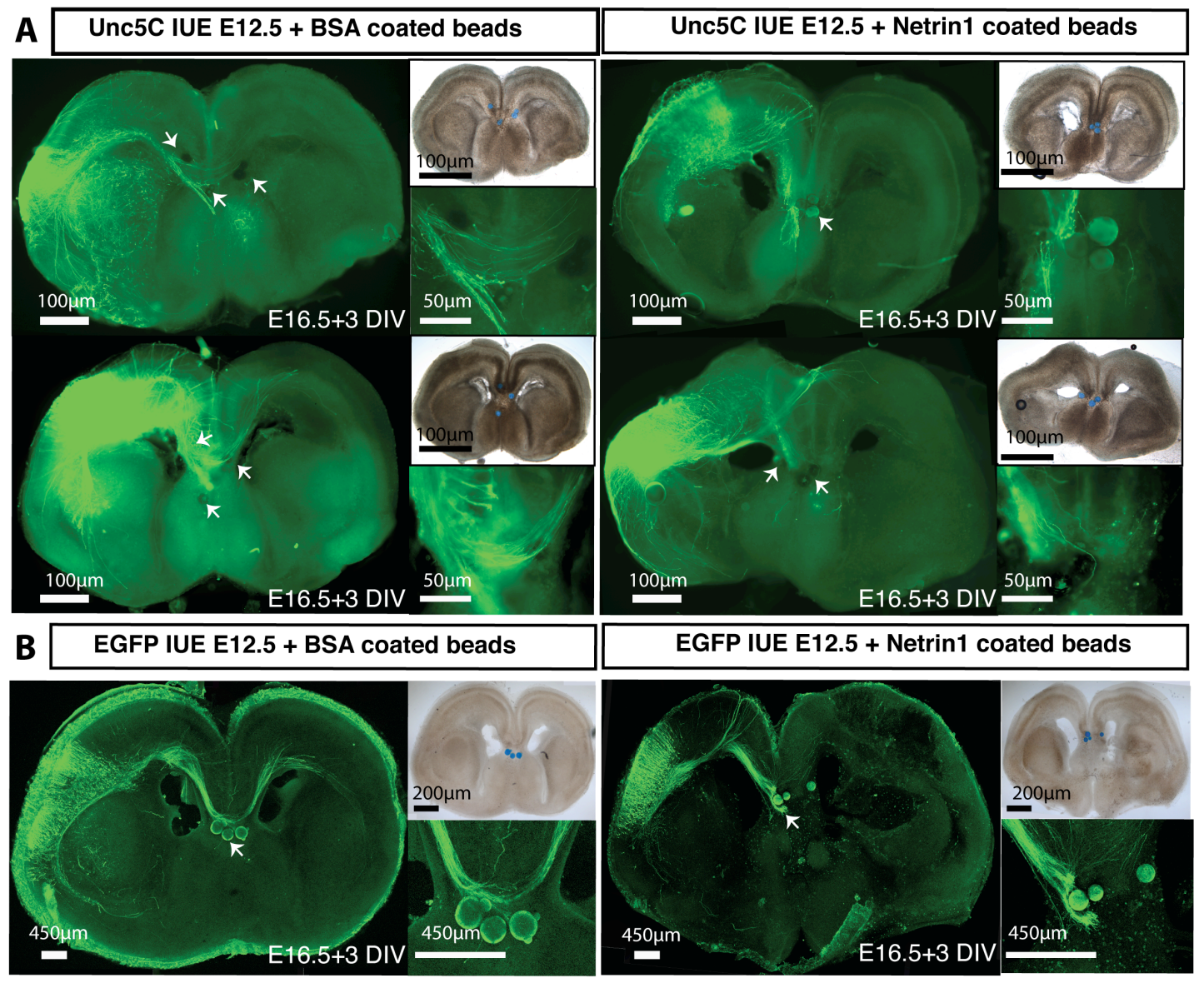

Figure 35. Netrin1 repels Unc5C positive neocortical axons.

Images showing the axonal trajectory of neurons electroporated with Unc5C (A) or EGFP (B) on encountering neutral BSA coated beads (arrows) or Netrin1 coated beads (arrows). Unc5Cpositive fibers are unaffected by BSA coated beads, placed along the midline, and continue along their initial trajectory to project into the contralateral hemisphere. Unc5C-positive fibers, upon encountering a Netrin1 source in the midline show clear repulsive behavior with fibers projecting either dorsal or ventral to their initial path, but none continuing into the contralateral hemisphere. Wild type axons electroporated with only EGFP (with endogenous Unc5C) are unaffected by the BSA beads and are repelled by the Netrin1 beads similar to the Unc5C over-expressing axons. Scale bar specified in the figure.

Conversely, when these Unc5C positive axon fibers encountered BSA soaked beads, they were unaffected and continued in their trajectory, crossing the midline and entering the contralateral hemisphere (Figure 35A). This experiment indicated that Unc5C expressing cortical neurons are indeed repelled by a Netrin1 source. By expressing only EGFP in cortical neurons, we 
tested our hypothesis that if callosal fibers express Unc5C endogenously, then they would also be repelled from a Netrin1 source. Again, these fibers failed to cross the midline upon encountering Netrin1, whereas BSA coated beads posed no hindrance to the midline crossing (Figure 35B).

\subsection{Misrouting of Satb2 positive axons in both Netrin1 and}

\section{Unc5C mutants.}

Since Unc5C is expressed in Satb2 positive neurons (Figure 28), and their axons are repelled by Netrin1 in vitro (Figure 35), we asked whether Netrin1 deletion would have a similar effect on callosal axons in vivo. In the developing telencephalon Netrin1 is expressed in the basal ganglia and ventral midline (Barallobre et al., 2000; Métin et al., 1997). We reasoned that the source of Netrin1 in the basal ganglia might normally repel the axons of Satb2 positive/Unc5C positive neurons from the internal capsule. We hypothesized that $S a t b 2$ positive neurons would misproject to sub-cortical targets in Netrin 1 deficient brains. To test this hypothesis, we placed crystals of the lipophilic tracer DiI at the cerebral peduncle of E17.5 Netrin1 hypomorph and wild type brains to back trace the neurons that project subcortically. After staining these sections with an antibody against Satb2, we counted the proportion of DiI-positive retrograde-labeled neurons that were also Satb2 positive (Figure 36A). We observed that the proportion of Satb2 positive- DiI labeled cells representing the proportion of Satb2-positive, subcortically projecting neurons, almost doubled in Netrin1 hypomorph brains compared to their wild type littermates (Figure 36B, 15.36\% in wild type compared to $27.33 \%$ in Netrin 1 hypomorph, $\mathrm{p}$-value $=0.011, \mathrm{n}=3$ ). 

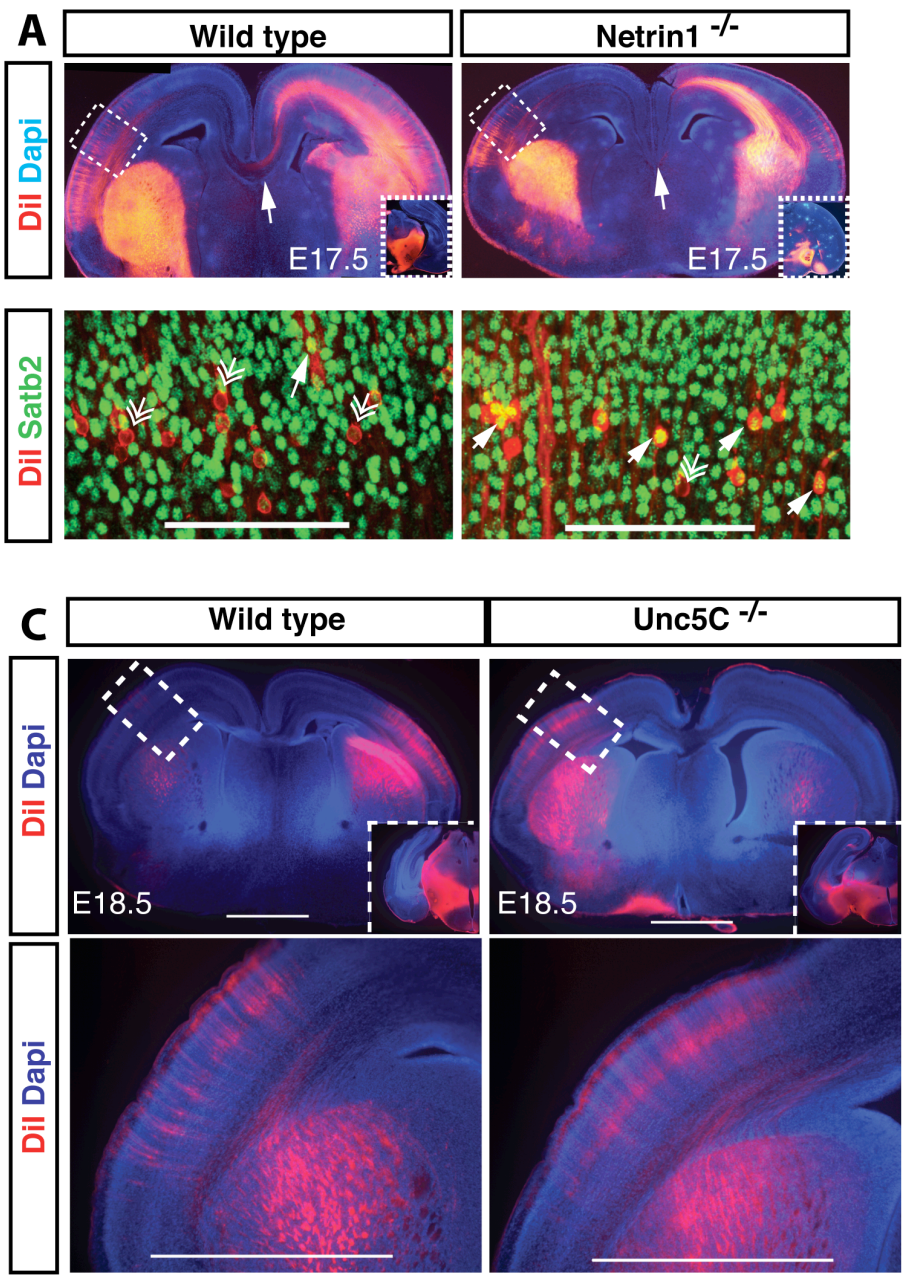

\section{$\mathbf{E}$}
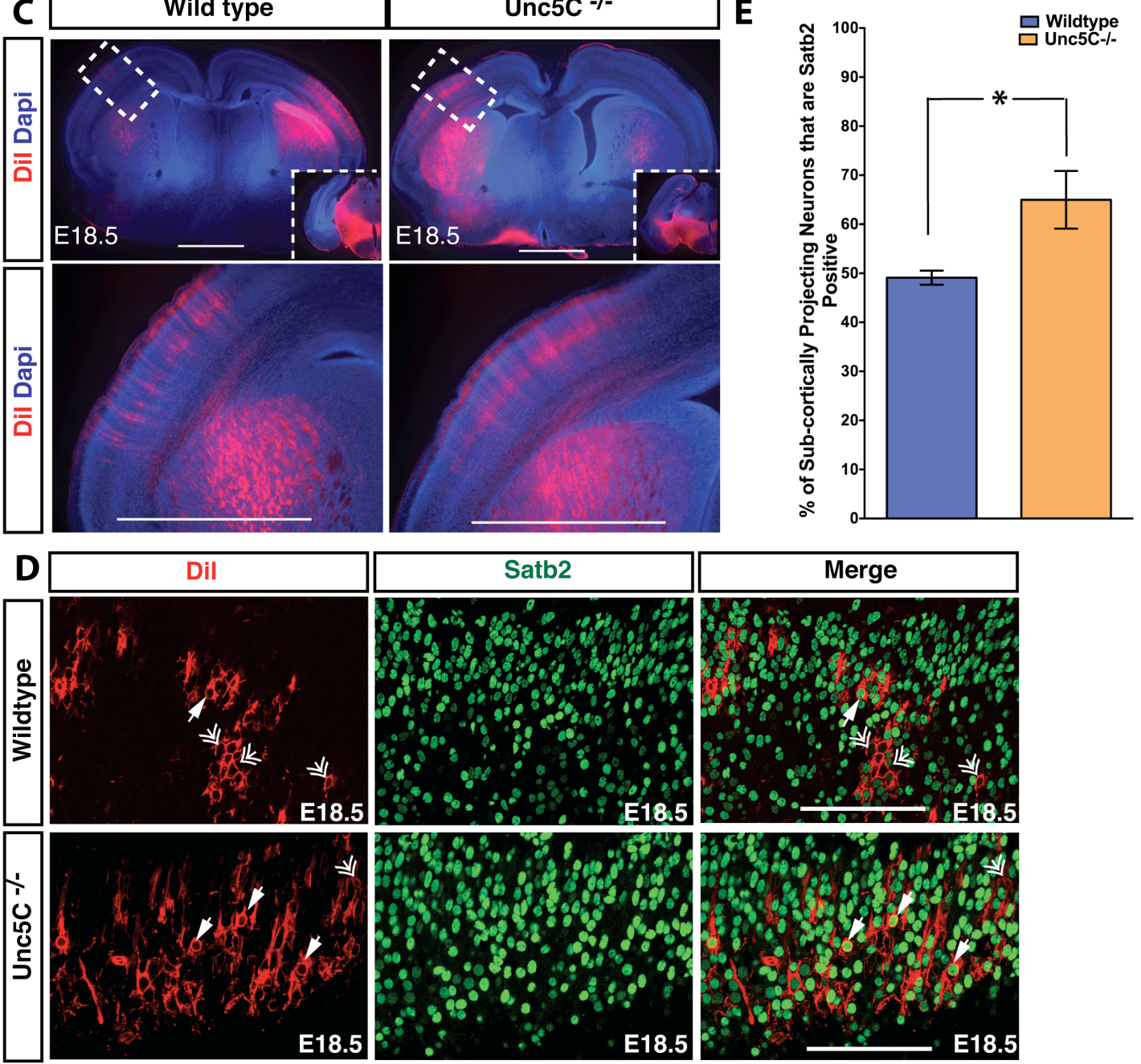

Figure 36. Satb2 cells are similarly misrouted to subcortical targets in Netrin1 and Unc5C mutants

(A) Retrograde labeling of subcerebrally projecting neurons by placing crystals of DiI in the cerebral peduncle of wild type and Netrin $1 \%$ brains. The smaller inset image show the respective position of the crystal placement. Back-labeled Dil-positive cells were co-localized with Satb2 in wild type and Netrin1 mutant brains. Arrows point towards double positive cells while double arrows highlight single DiI-positive cells. (B) Quantification of the percentage of DiI-positive cells that are Satb2-positive; $15.36 \%$ in wild type versus $27.33 \%$ in E17.5 Netrin1 mutant, p-value $=0.011, n=3$. Error bars represent s.e.m. (C) Crystals of the 
lipophilic tracer DiI were placed in the cerebral peduncle of wild type and Unc5C- brains to retrogradely label cortical neurons projecting sub-cerebrally. The smaller inset images show the location of the crystals placed in the cerebral peduncle. Higher magnification image of the boxed region show layer V neurons back-labeled in the cortex. (D) Co-localization of DiIpositive and Satb2-positive cells in the neocortex. Arrows indicate double positive cells, while double arrows indicate single DiI-positive cells. Scale bar $=100 \mu \mathrm{m}$. (E) Quantification of the proportion of sub-cortically projecting neurons that are Satb2 positive; $49.10 \%$ in wild type versus $64.98 \%$ in E18.5 Unc5C--; $p$-value $=0.0286, \mathrm{n}=3$. Error bars represent s.e.m.

We also tested whether Unc5C ablation in neocortical neurons would cause a similar effect. In contrast to the acallosal Netrin1 mutant, Unc5C mutants display callosal midline crossing fibers. We therefore tested whether some axons that would normally project to the CC are redirected to the internal capsule in the Unc5C mutant. We performed similar DiI retrograde-labeling experiments in Unc5C-/ brains and counted the number of Satb2 positive cells projecting to the internal capsule (Figure 36C, D). We found that the proportion of Satb2 positive, sub-cortically projecting cells was $16 \%$ higher in the Unc5C mutants compared to wild type littermate brains (Figure 36E; $49.1 \%$ in wild type versus $64.98 \%$ in Unc5C $\%$ brains, $p$-value $=0.0286, n=3$ ). Therefore, although callosal axons cross the midline in the Unc5C mutant, more Satb2-positive axons project to the internal capsule in both these mutants. This finding indicates that a Netrin1-Unc5C interaction plays an important role in determining the choice of axonal target projection (medially versus laterally), in a cell-autonomous manner.

\subsection{Satb2 is a direct repressor of DCC transcription.}

Since restoring the expression of Unc5C in Satb2 $\%$ mutants lead to only a partial rescue of the $\mathrm{CC}$, we tested whether additional axon guidance molecules in this pathway that are important in CC formation displayed altered expression in Satb2\% brains. Like Unc5C, DCC is also a receptor for Netrin1, but has been shown to mediate axonal attraction (Chan et al., 1996b; Deiner et al., 1997; Fazeli et al., 1997). 


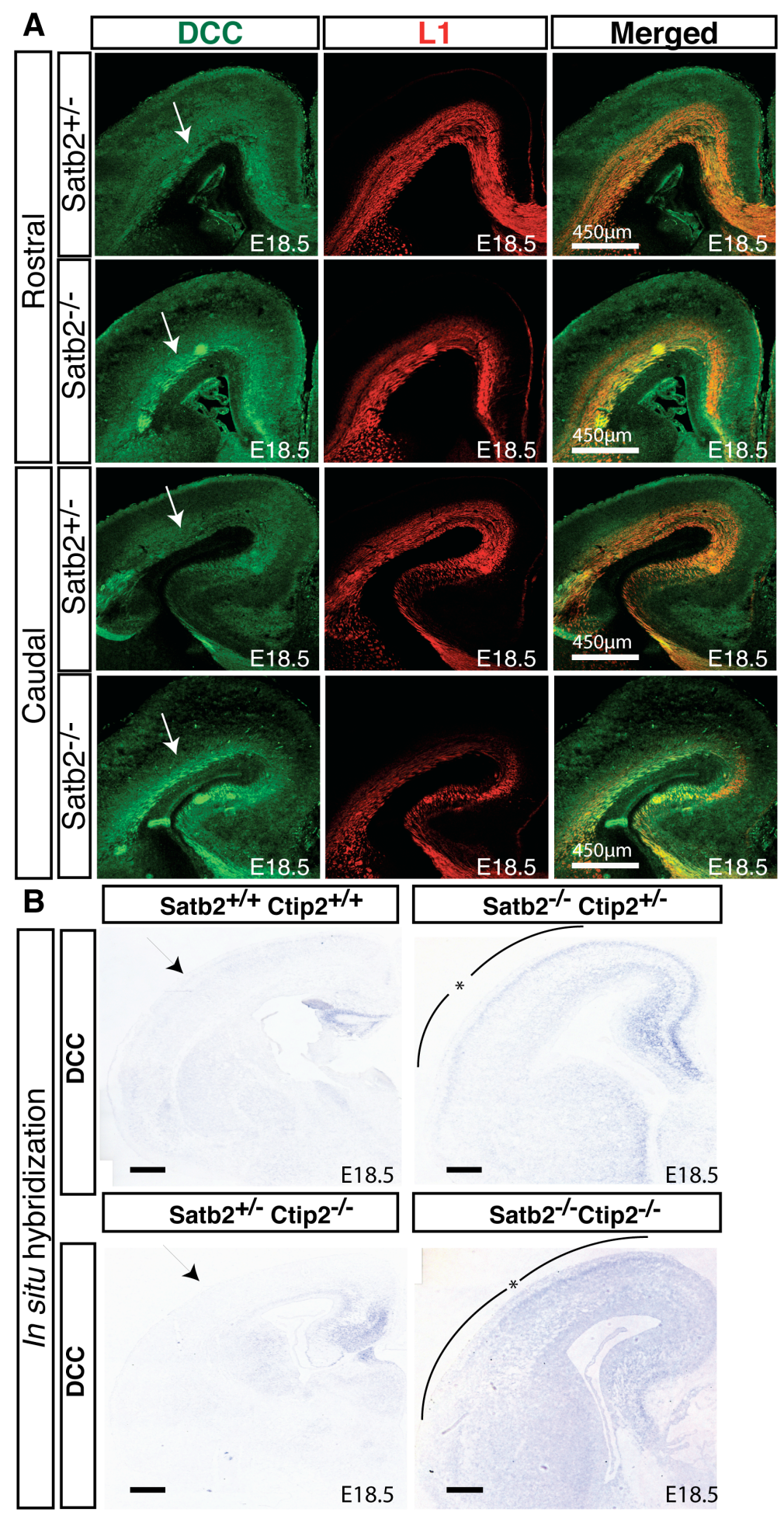

Figure 37. DCC is up-regulated in the Satb2 $\%$ mutant and Satb2 $\%$;Ctip2 $\%$ double mutant cortex

(A) Immunohistochemical staining for DCC in rostral and caudal sections show an upregulation of DCC expression in Satb2- mutant brains as compared to wild type littermates. Arrows point to the region of altered DCC expression between wild type and Satb2\% sections. (B) In situ hybridization reflects an up-regulation of DCC in E18.5 Satb2\% cortex when compared to Satb2 heterozygous brains. Ctip $2-$ mutant did not show any alteration in DCC expression when compared to controls. Satb2-\%Ctip2 $\%$ double mutant showed comparable expression to the Satb2 - mutant. Scale bar $=100 \mu \mathrm{m}$. 
We performed immunohistochemical staining and in situ hybridization for DCC in wild type as well as Satb2\% cortices at E18.5. Both, DCC protein and mRNA expression was upregulated in Satb2\% brains as compared to wild type littermates (Figure 37A, B). In contrast, no changes in DCC expression were observed between the control and Ctip2 $2^{--}$or between the Satb2-; Ctip2\% double mutant and Satb2- single mutant indicating that Ctip2 did not effect DCC expression (Figure 37B).

In the wild type cortex, DCC demonstrated a very dynamic pattern of expression. In contrast to Unc5C expression, DCC mRNA expression is high in the cortical plate at E14.5 (Figure 38A) and below detection levels at E18.5. Only a low level of DCC protein expression was observed in the white matter at E18.5 (Figure 37B). In order to investigate if Satb2 could regulate DCC expression, we over-expressed $S a t b 2$ and $S k i$ prematurely in wild type cortex at E11.5 and analyzed the brains for DCC expression at E14.5. Again, Ski was co-electroporated to enable Satb2 to recruit the transcription repressor complex (Baranek \& Atanasoski, 2012; Baranek et al., 2012). At E14.5, since there are few endogenous Satb2 positive cells in the cortex and since DCC expression is high in the cortical plate, we assumed that any alteration in DCC expression would be observable. In this experiment DCC expression was significantly lower in the electroporated region compared to the nonelectroporated hemisphere $(n=2)$. In contrast, EGFP electroporation alone did not alter the expression of DCC $(\mathrm{n}=2)$ (Figure 38A).

To investigate whether Satb2 could directly control DCC expression, we performed ChIP assays for two putative Satb2 binding sites (MAR1 (matrix attachment region1) and MAR2) upstream of the DCC transcription initiation site (Figure 38B). These regions are AT rich and could therefore serve as Satb2 binding sites. We observed a significant enrichment of the region more proximal to the TSS (MAR1) in wild type tissue as compared to Satb2 mutant tissue, which served as the negative control $(5.27 \pm 2.75$ fold enrichment in $\mathrm{n}=6$ wild type compared to $1.72 \pm 0.015$ in $\mathrm{n}=2$ Satb2\% mutants, p-value= $0.025)$. 


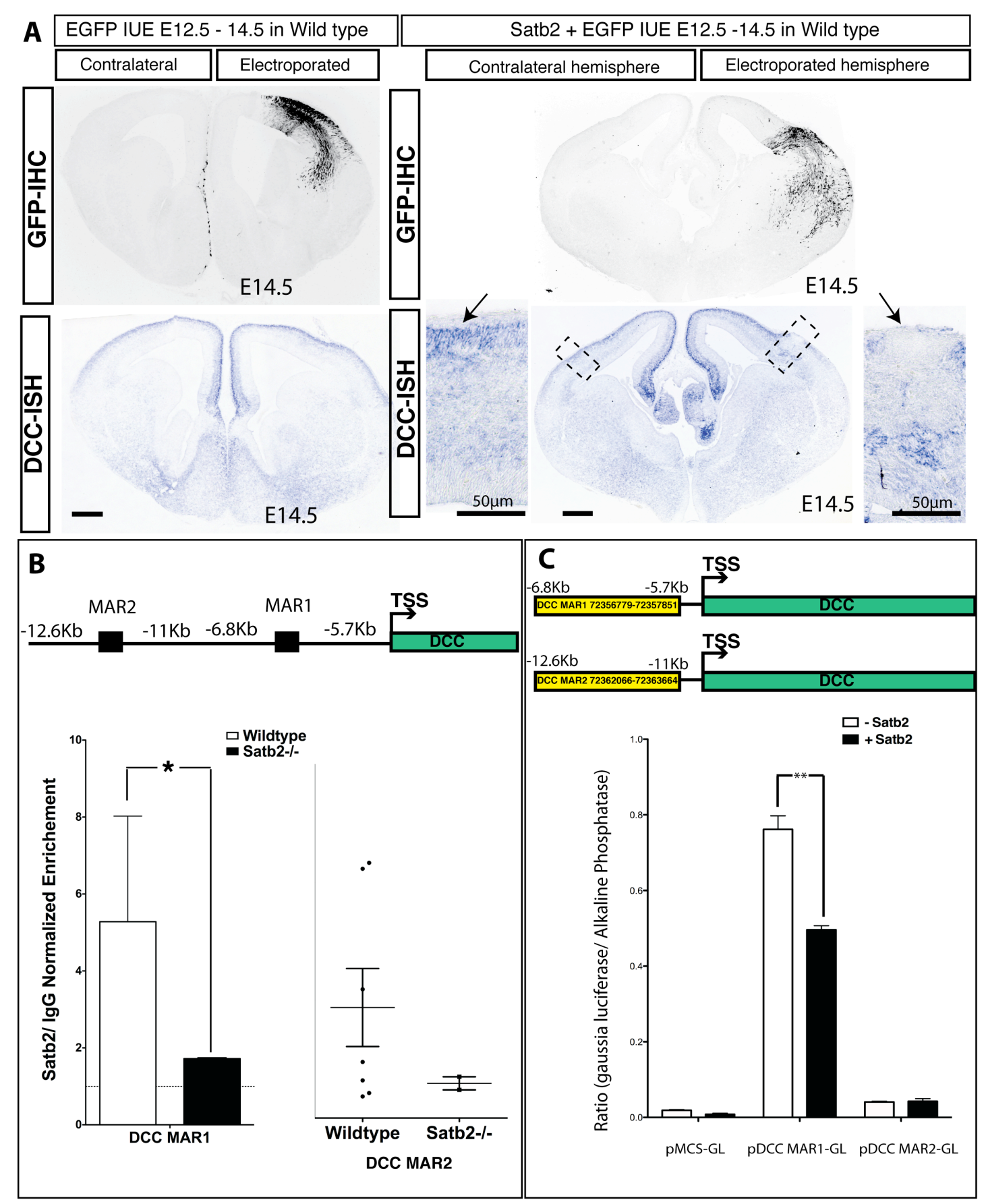

Figure 38. Satb2 is a direct transcriptional repressor of DCC expression

(A) Series of images show DCC expression in E14.5 wild type embryos in cells electroporated with either Satb2/Ski/EGFP at E11.5 or with EGFP alone at E12.5. GFP immunohistochemistry delineates the region electroporated. DCC in situ hybridization demonstrates a down regulation of DCC mRNA within cells electroporated with Satb2/Ski compared to DCC expression in the contralateral hemisphere, or in the control electroporation. (B) Schematic diagram showing two putative binding MAR sites for Satb2 upstream of the DCC transcription initiation site. Chromatin-immunoprecipitation from P0 cortices showed a $5.27 \pm$ 2.75 fold enrichment in $n=6$ wild type compared to $1.72 \pm 0.015$ in $n=2$ Satb2 $\%$ mutants for DCC MAR1, p-value $=0.025$ Student's t-test and for DCC MAR2, $\mathrm{n}=7$ wild type showed a range of $0.91-10.83$ fold enrichment with an average of $4.69 \pm 4.38$, as compared to $1.47 \pm 0.39$ in $\mathrm{n}=2$ Satb2 - mutants, $\mathrm{p}$-value $=0.10$ Student's t-test $(\mathrm{C})$ Luciferase assay to demonstrate that Satb2 binds to the DCC genomic region and represses its expression. In the presence of full length Satb2, DCC MAR1 showed a 1.5 fold decrease in luminescence ratio while DCC MAR2 
did not shown any change (Student's t-test, $\mathrm{p}$-value $=0.0035$ ). Scale bar $=100 \mu \mathrm{m}$, unless specified differently in the figure.

In contrast, enrichment for the second MAR region (MAR2) was highly variable indicating that Satb2 might bind very weakly to this promoter region ( $\mathrm{n}=7$ wild type showed a range of 0.91-10.83 fold enrichment with an average of $4.69 \pm 4.38$, as compared to $1.47 \pm 0.39$ in $n=2$ Satb2 $\%$ mutants, $p$-value $=$ 0.10). To further investigate the functional significance of this MAR binding, we cloned the two genomic regions, DCC MAR1 and DCC MAR2, into a Gaussia Luciferase vector and transfected them into COS cells either in the presence or absence of Satb2. DCC MAR1 (chromosome 18 7235677972357851) showed a 1.5 fold decrease in transcription levels when coexpressed with Satb2 (Figure 38C, n=3, p-value=0.0035). DCC MAR2 (chromosome 18 72362066-72363664) did not show any change in transcription levels upon Satb2 co-expression (Figure 38C, n=3, pvalue $=0.742$ ). Taken together, these findings suggest that Satb2 can directly repress DCC expression and might be another factor influencing corticocortical versus cortico-subcortical axon targeting choice.

\subsection{Downregulation of DCC in Satb2 mutants results in partial rescue of the CC}

To test the hypothesis that DCC up-regulation contributed to the misrouting of callosal axons in Satb2 deficient brains, we tested whether downregulating DCC in the Satb2 mutant could restore callosal projections. We tested the downregulation efficiency of four different shRNA constructs by transfecting DCC shRNA or the scrambled shRNA together with a DCC expression plasmid in HEK293T cells and checking protein levels by Western blot (Figure 39A). Based on these experiments we selected two DCC shRNA constructs (clone 2 and 4) and co-electroporated them with Venus-GFP at E12.5 in Satb2\% and wild type cortices. 
A
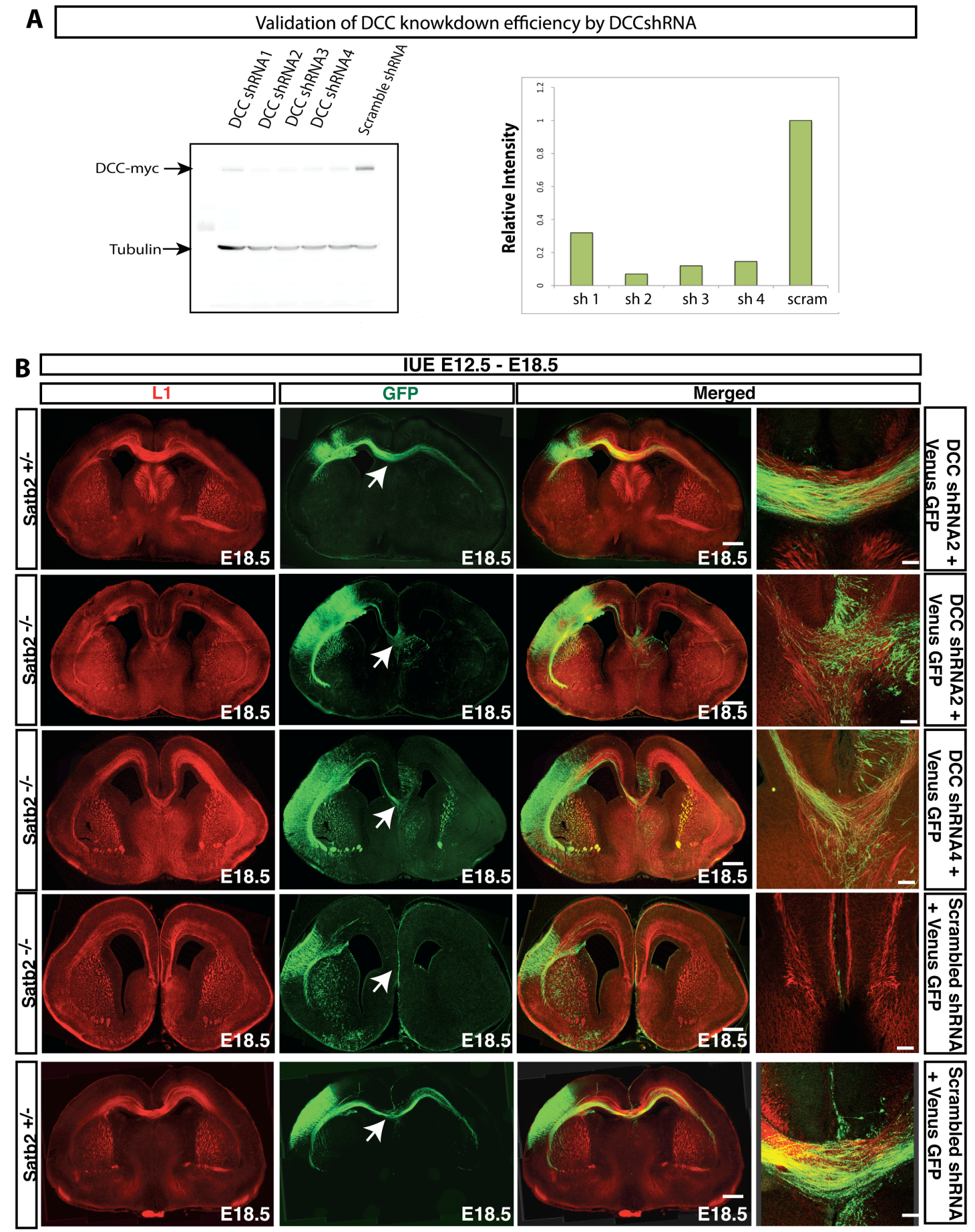

IUE E12.5 - E18.5

Figure 39. Down-regulating DCC in the Satb2\% cortex leads to a partial rescue of the corpus callosum.

(A) The efficiency of DCC knockdown was verified in HEK293T cells. Four different shRNAs were tested for their efficiency in DCC knockdown. A scrambled shRNA was used as a control. Western blot analysis shows a clear reduction in DCC protein levels in all four shRNAs compared to the scrambled shRNA. Quantification shows relative protein levels of DCC to tubulin. DCC shRNA 2 and 4 proved most efficient in knocking down DCC, and were thus used in the experiments. (B) Images show a series of experiments, wherein in utero electroporation was done at E12.5 and the brains harvested and analyzed at E18.5. In utero electroporation of DCC shRNA and Venus GFP do not seem to have a major effect on the formation of the CC in wild type embryos. In utero electroporation of two different DCC shRNAs (shRNA2 and shRNA4) along with Venus GFP show a reproducible partial rescue of 
the CC in Satb2\% embryos. In utero electroporation of a scrambled shRNA and Venus GFP cannot rescue the CC in Satb2\% embryos. Arrows point towards the dorsal midline. Scale bar= $450 \mu \mathrm{m}$ for low magnification images and $100 \mu \mathrm{m}$ for high magnification images.

While callosal axons crossed the midline in wild type brains electroporated with either the DCC shRNA or the scrambled shRNA, no midline crossing fibers were observed when scrambled shRNA was electroporated in Satb2\% brains $(\mathrm{n}=2)$. In contrast, neurons electroporated with DCC shRNA in the Satb2\% cortex now extended towards and crossed the midline $(n=3$, Figure 39B). The rescue was however again only partial (Quantification of CC rescue- Figure 43). Together, these results indicate that Satb2 directly represses the expression of DCC and that an up-regulation of DCC in the Satb2- cortex could contribute to the lack of callosal axons crossing the midline.

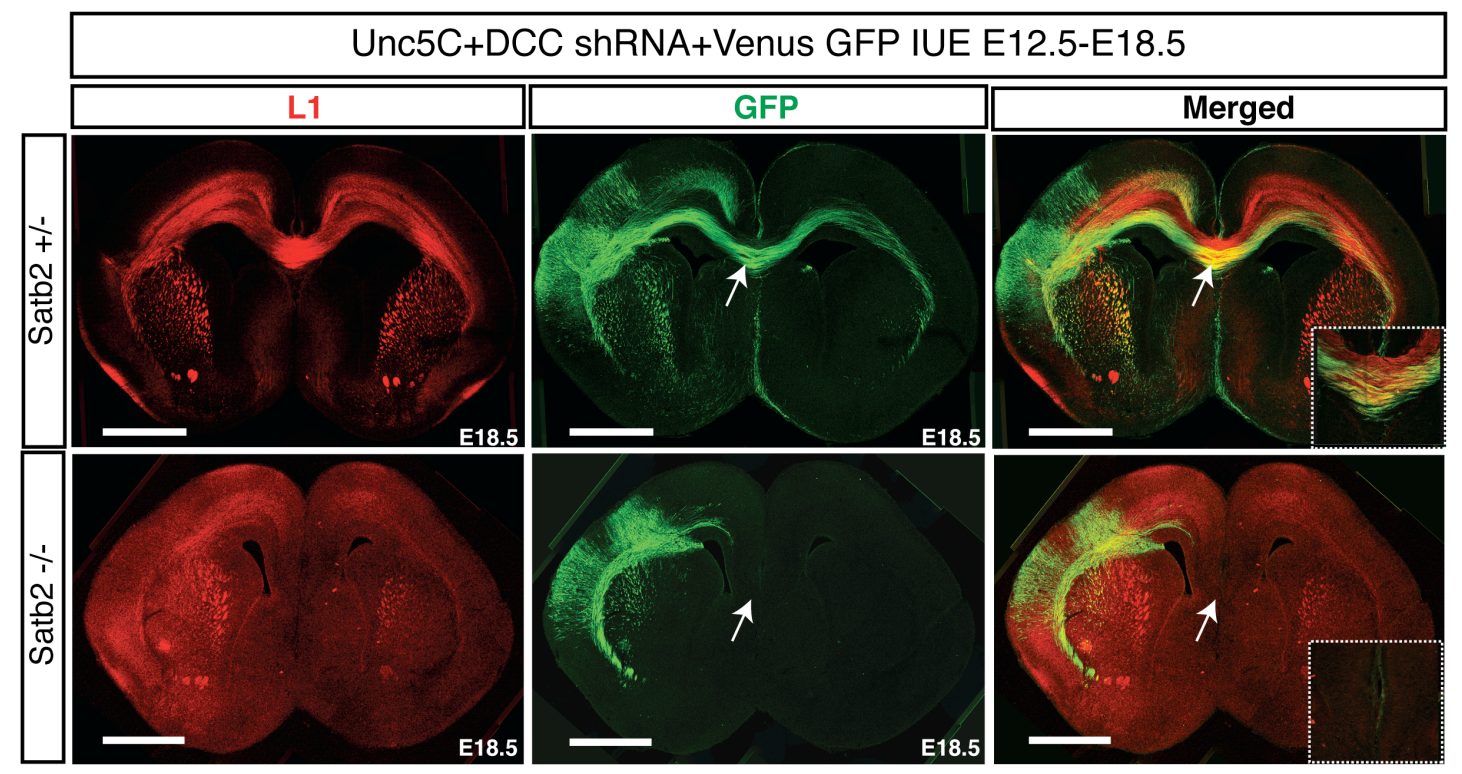

Figure 40. Over-expression of $U n c 5 C$ with the simultaneous down-regulation of $D C C$ does not rescue the corpus callosum.

Images show experiments, wherein Unc5C, DCC shRNA and Venus GFP were coelectroporated at E12.5 and the brains harvested and analyzed at E18.5. Wild type embryos display a normal CC at E18.5. The CC was not rescued when Unc5C and DCC shRNA were co-electroporated in Satb2\% embryos. Inset images show higher magnification of the midline. Scale bar $=450 \mu \mathrm{m}$.

In order to test whether Unc5C and DCC act as parallel pathways and the extent of CC restoration in Satb2 mutants could be enhanced by simultaneously down-regulation DCC and up-regulating Unc5C in the Satb2 mutant, we coexpressed DCC shRNA and Unc5C overexpression construct 
together with $e G F P$ in Satb2 $\%$ mutants. Surprisingly, in these experiments we did not detect any CC restoration (Figure 40).

\section{$\underline{3.36 \text { Netrin1/DCC/Unc5C interactions regulate callosal }}$}

\section{projections of only deep layer neurons}

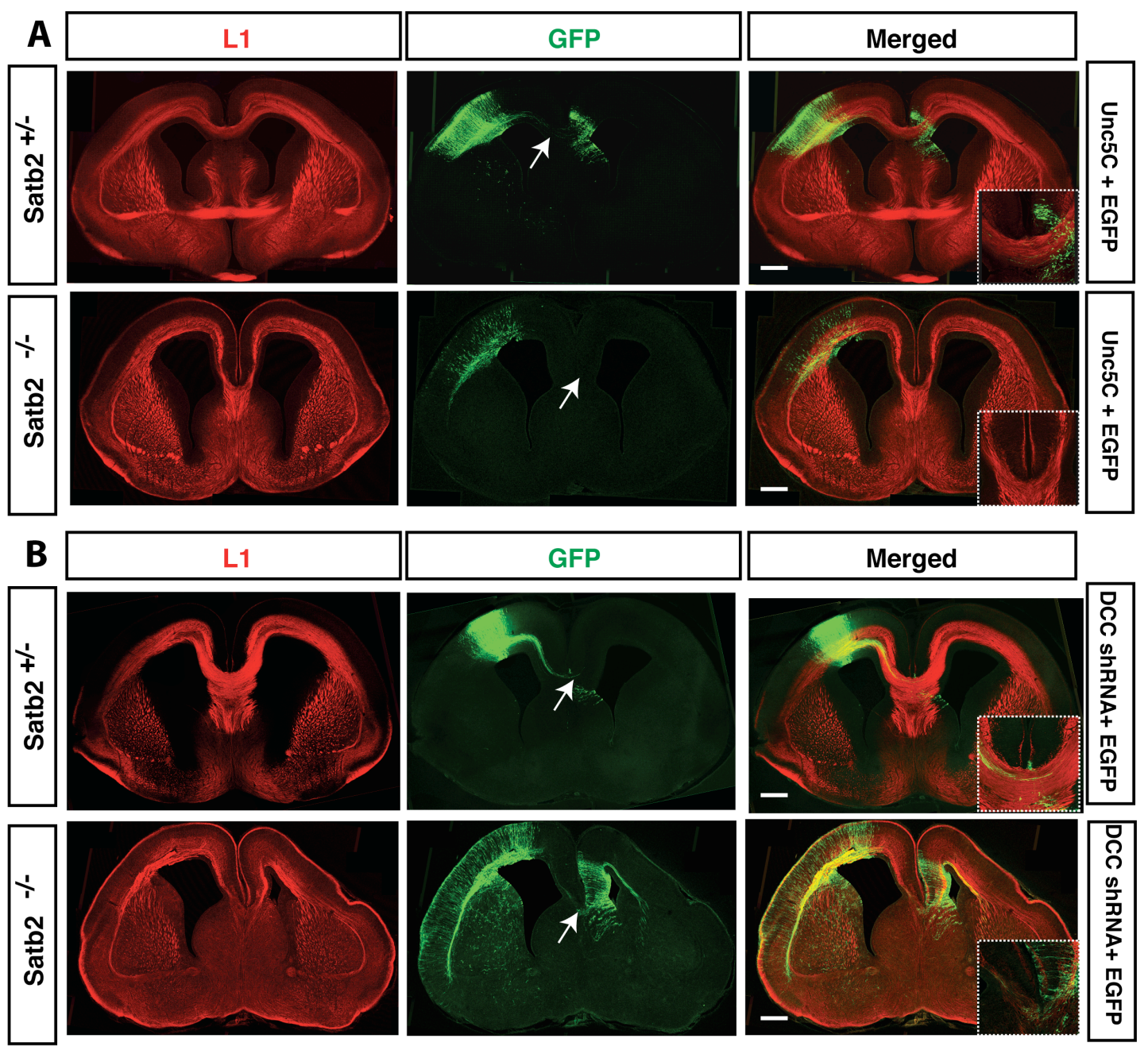

Figure 41. Over-expression of Unc5C or down-regulation of DCC at E14.5 does not rescue the CC in Satb2\% mutants

(A) Over-expression of Unc5C at E14.5 by in utero electroporation in Satb2\% mutants does not rescue the CC, whereas wild type littermates show normal callosum. (B) Down regulation of DCC by shRNA electroporation at E14.5 does not rescue the CC in Satb2\% mutants, whereas wild type littermates show normal callosum. Inset images show higher magnification of the midline. Scale bar $=450 \mu \mathrm{m}$.

Callosal neurons are a heterogeneous population of neurons (B J Molyneaux et al., 2009). The majority of callosal axons are from upper layer neurons, 
hence in order to test if upper layer neurons also show dependency on Satb2/Ctip2 and hence DCC/Unc5C for making the choice between taking a medial or a corticofugal trajectory, we electroporated Unc5C (Figure 41A) or DCC shRNA (Figure 41B) in Satb2\% cortex at E14.5 and harvested the brains at E18.5. The later stage of electroporation ensures targeting of mainly the upper layer neurons. We observed that both the experiments did not yield in a rescue of the $\mathrm{CC}$, and no fibers could be observed taking the medial path. Hence, with respect to the molecular mechanisms described here, it could be concluded that deep neocortical layers, born before E14.5, are dependent upon the Netrin1/Unc5C/DCC pathway. However, upper layer neurons, born after E14.5 probably require pathway(s) other than Netrin1/Unc5C/DCC.

\subsection{Satb2 has limited capacity to restore the corpus callosum through cell intrinsic mechanisms}

The lack of a complete restoration of the CC in Satb2 mutants after Unc5C upregulation or DCC down-regulation, questioned the extent of the cell autonomous contribution of Satb2 to CC formation. We therefore electroporated a Satb2 expression plasmid together with EGFP into Satb2\%brains at E12.5. At E18.5 we tested the ability of the electroporated Satb2 in repressing Ctip2 and found an absence of Ctip2 from the electroporated cells (Figure 42A). In the electroporated brains we observed that although the electroporated neurons in the Satb2\% mutants were able to form a CC, the rescue of the CC was again only partial, similar to the rescues obtained in case of either the Satb2\%;Ctip2\% double mutants, Unc5C over-expression or DCC down-regulation in the Satb2\% mutants (n=2, Figure 42B, Quantification of CC rescue - Figure 43). A similar principal seems to be underlying all of these scenarios as Satb2 upon ectopic over-expression in wild type cortices can lead to Unc5C over expression (Figure 28B) and DCC downregulation (Figure 38A). Again, we did not observe any rescue of the CC upon over-expression of Satb2 at later stages (E14.5, Figure 42C). 

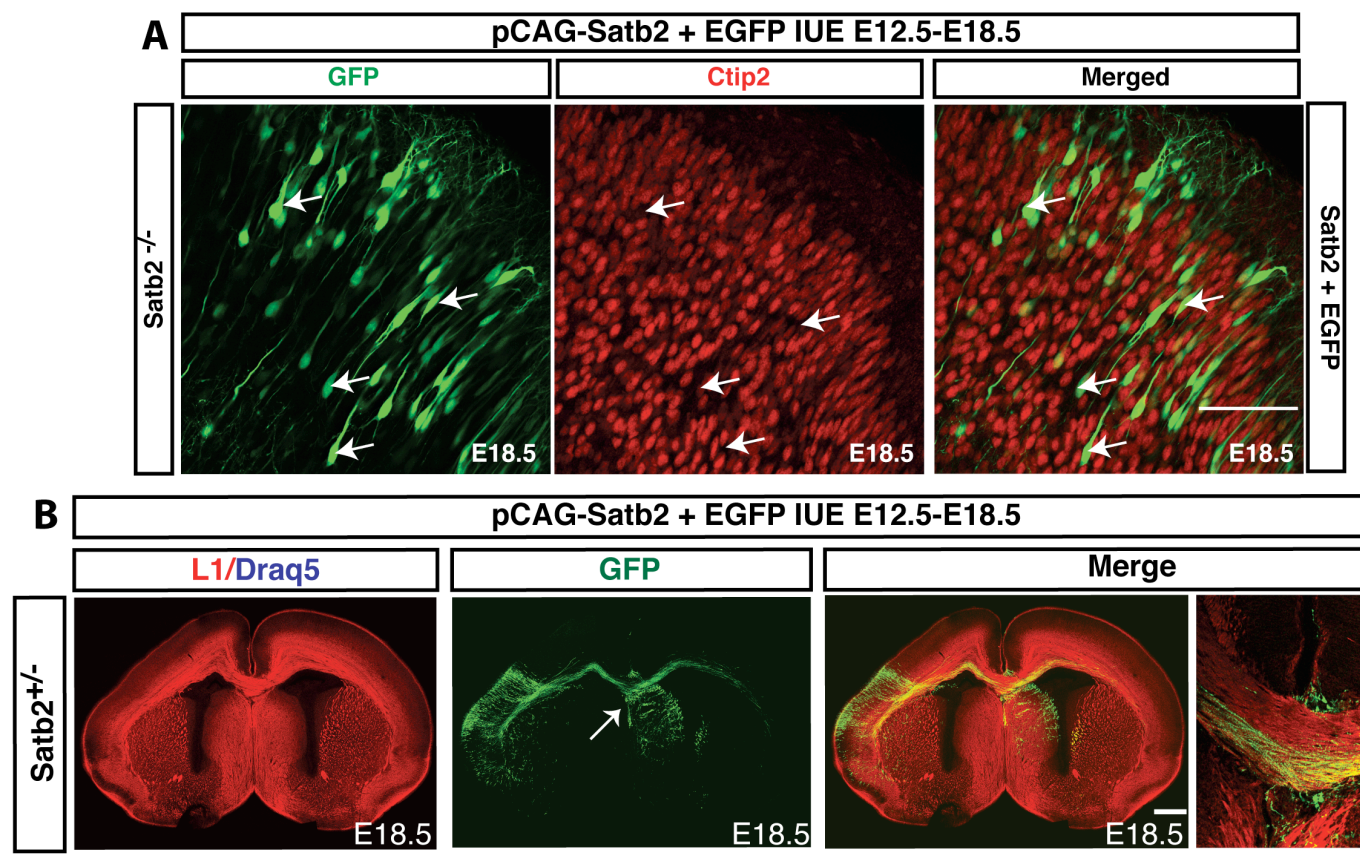

pCAG-Satb2 + EGFP IUE E12.5-E18.5
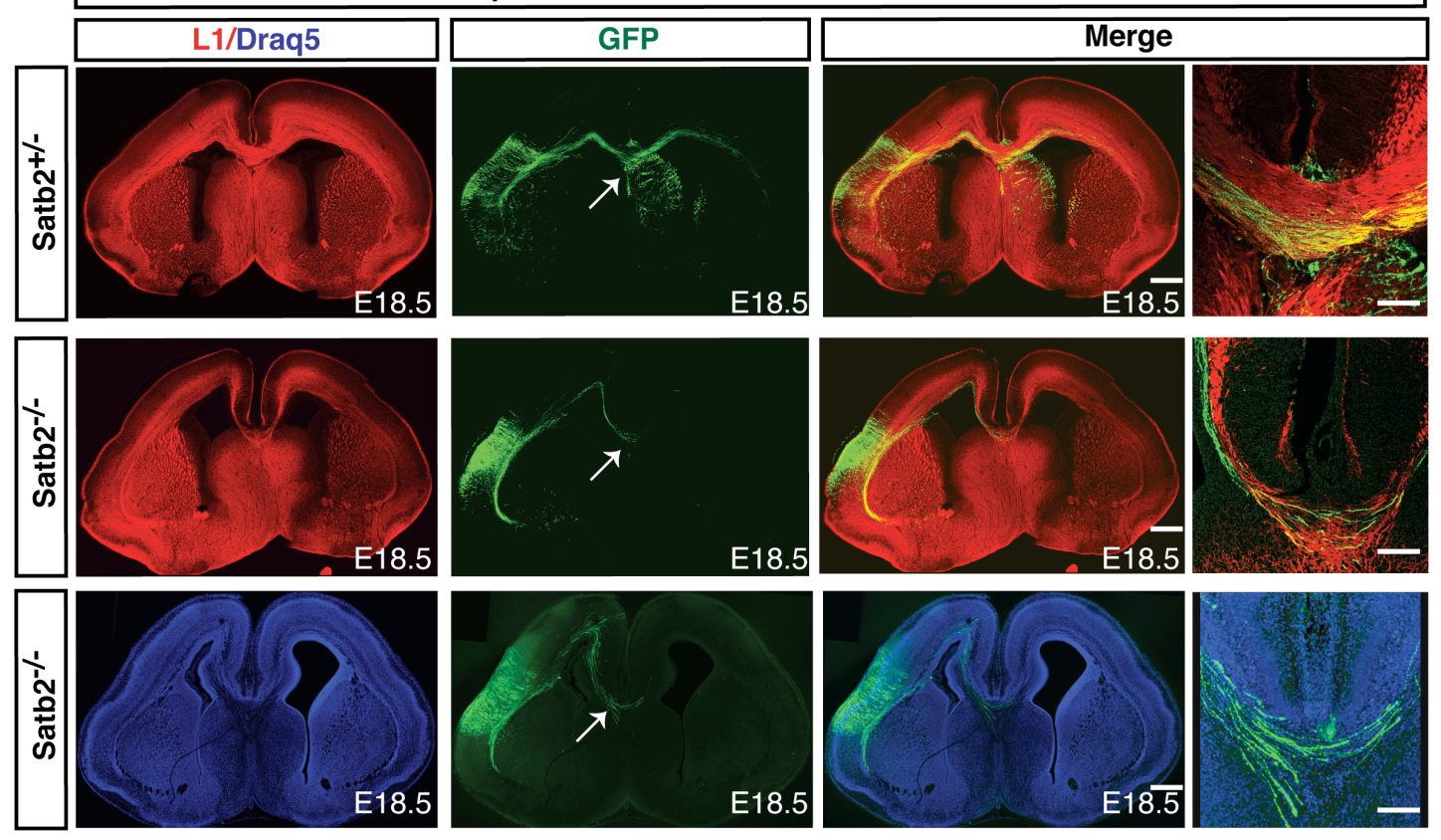

\section{C}

pCAG-Satb2 + EGFP IUE E14.5-E18.5

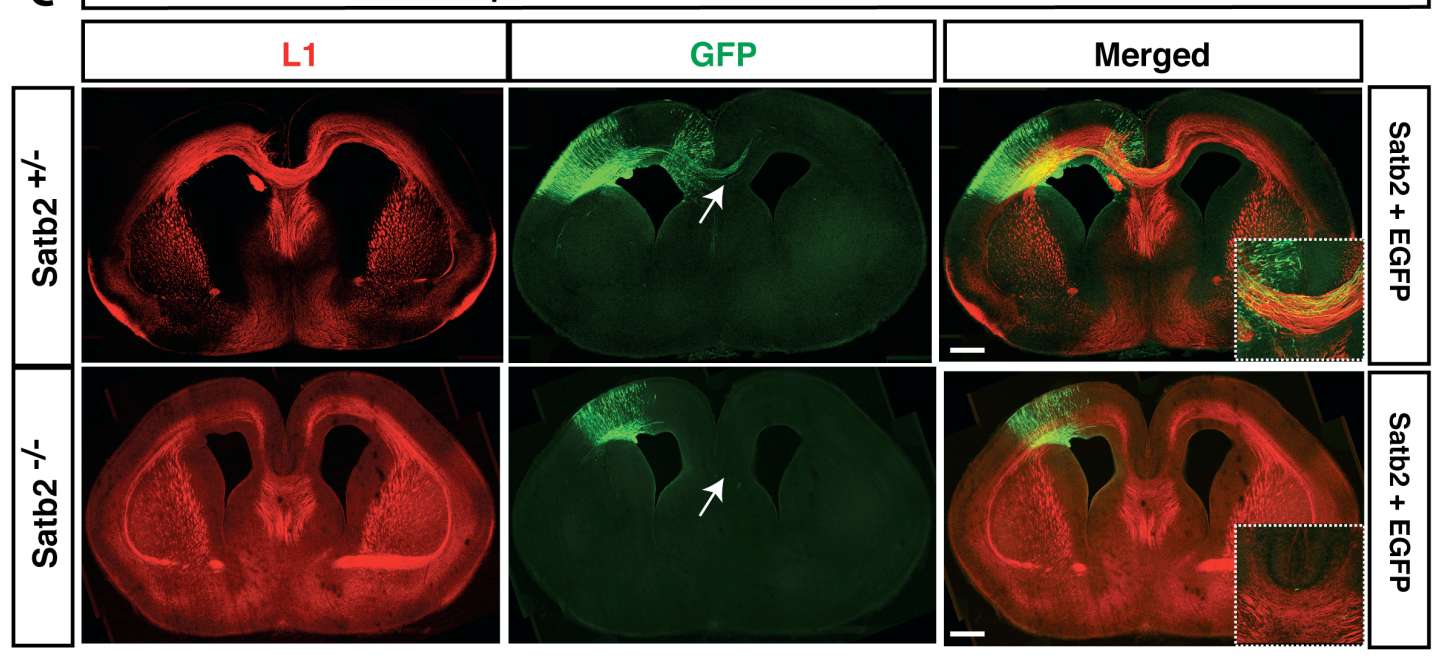

Figure 42. Satb2 electroporation in $S a t b 2 \%$ cortex partially rescues the corpus callosum.

(A) In utero electroporation of Satb2 in Satb2\% cortex results in a down regulation of Ctip2 expression in the electroporated cells, verifying the activity of Satb2 in these cells. Scale bar $=100 \mu \mathrm{m}$. (B) In utero electroporation of plasmids encoding for Satb2 and EGFP in wild type and Satb2\% embryos at E12.5, harvested at E18.5 followed by immunohistochemical staining for the neuronal adhesion molecule L1, show a partial rescue of the CC in Satb2\% mutants. Alternatively, sections were counter stained with the far-read nuclear marker Draq5. Arrows 
indicate fibers crossing the midline. Scale bar $=450 \mu \mathrm{m}$ for low magnification images and $100 \mu \mathrm{m}$ for high magnification images. (C) Over-expression of Satb2 at E14.5 does not rescue the CC in Satb2\% mutants, whereas wild type littermates show normal CC. Inset images show higher magnification of the midline. Scale bar $=450 \mu \mathrm{m}$. Inset images show higher magnification of the midline.

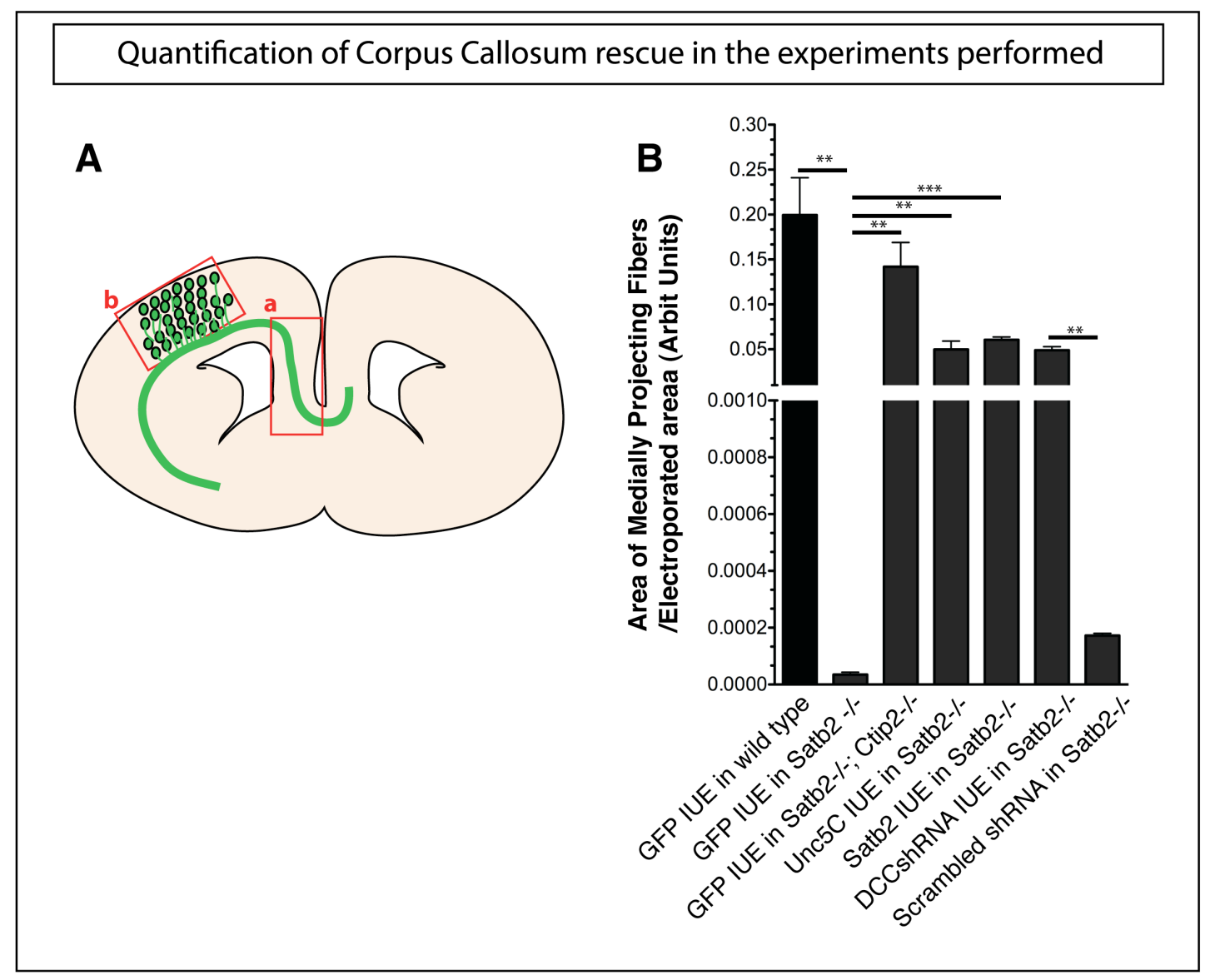

Figure 43. Quantification of CC with respect to efficiency of electroporation in wild type and Satb2\% mutant

(A) In order to quantify the extent of rescue of medial projections in the in utero experiments performed, ratio of the fluorescent area of the medially projecting fibers in the ipsilateral hemisphere (within box a) to the fluorescent area of the cells electroporated in the cortical plate (within box $b$ ) was measured. The size of box ' $b$ ' varied depending upon the extent of electroporation. The size of box ' $a$ ' was adjusted so as to enclose the extent of medially projecting fibers after they make the dorsal turn within the ipsilateral cortex till the midline. (B) The in utero-experiments with GFP in wild type, GFP in Satb2 $-\%$, GFP in Satb2 $-\%$; Ctip2 $2-$, Unc5C in Satb2- Satb2 in Satb2 $\%$, DCC shRNA in Satb2 $\%$ and Scrambled shRNA in Satb2 $\%$ were quantified. All values are represented as mean \pm SEM. All $p$ values are calculated by student's $t$ test. GFP in wild type $(\mathrm{n}=3)=0.199 \pm 0.0415$, GFP in Satb2 $\%(\mathrm{n}=3)=0.00003 \pm 8.26 \times 10^{-}$ 6 , $\mathrm{p}$ value $=0.0086$, GFP in Satb2 $-;$ Ctip $\%(\mathrm{n}=2)=0.1419 \pm 0.027$, $\mathrm{p}$ value $=0.0058$, Unc5C overexpression in Satb2 $\%(\mathrm{n}=3)=0.0498 \pm 0.009$, $\mathrm{p}$ value $=0.0058$, Satb2 electroporation in Satb2 $\%$ $(\mathrm{n}=2)=0.0606 \pm 0.003, \mathrm{p}$ value $=0.00012, D C C$ shRNA in Satb2 $\%(\mathrm{n}=3)=0.0489 \pm 0.003$, compared to scrambled electroporation in the Satb2- $(\mathrm{n}=2)=0.00017 \pm 7.83 \times 10^{-6}, \mathrm{p}$ value $=0.00023$. 
Together, the above experiments suggest that Satb2 and Ctip2 play a crucial role in the decision of axonal projections to take a medial route and form the corpus callosum or take a sub/cortical route. The axons make this choice with the help of the axon guidance molecules DCC and Unc5C that respond to Netrin1, where Satb2 transcriptionally regulates DCC and Ctip2 regulates Unc5C expression. 


\section{DISCUSSION}

Evolution of the brain has witnessed a consistent growth in both the number of neocortical neurons as well as in the connections that these neurons make. This robust increase in size and circuitry has tremendously augmented the variety as well as the complexity of the tasks the brain is capable of executing. Understanding the developmental mechanisms involved in establishing these connections hence forms an integral part of understanding the functions of the brain as a whole (Geschwind \& Rakic, 2013). To understand how neurons extend their axons, elongate at a particular rate, branch out at particular regions and finally stop growing at a critical time is essential not only for understanding the development of the nervous system but also to understand the mechanisms involved in regrowth of axons after injury (Goldberg, 2003). In this study we have investigated the contribution of three different transcription factors, namely Sip1, Satb2 and Ctip2 and the regulatory mechanisms downstream of these factors involved in establishing cortical projections.

Sip1 is a SMAD binding protein, which together with a cohort of cofactors has been shown to behave as both a transcriptional repressor as well as a transcriptional activator depending on the cellular and genomic context (Comijn et al., 2001; Postigo \& Dean, 2000; Postigo et al., 2003; Verschueren et al., 1999; Yoshimoto et al., 2005). In the neocortex Sip1 is expressed exclusively in the postmitotic compartment of the cortex and the expression of the protein is detectable when the first set of neurons poppulate the cortical plate and the expression expands as the cortical plate grows (Seuntjens et al., 2009). Almost all neocortical neurons express Sip1 indicating that it could potentially play a global role with respect to development of the different types of neocortical neurons and their connections. In this study we have investigated the various cortical projection defects in the Sip1f/fl NexCre conditional mutant and 
dissected out the cell intrinsic and cell extrinsic mechanisms with which Sip1 exerts its control over establishing cortical circuitry.

Unlike Sip1, Satb2 and Ctip2 are expressed in very specific sub sets of neocortical neurons. While Satb2 is expressed mostly in callosally projecting neurons in layer II, III and layer V, Ctip2 is expressed in neurons that project through the CST that form the majority of layer V neurons (Alcamo et al., 2008; Arlotta et al., 2005; Britanova et al., 2008). In the mouse brain, though the majority of callosally projecting neurons lie in layers II, and III, some of the layer V neurons also project through the CC (Ivy \& Killackey, 1981). Hence, in the mouse, layer $\mathrm{V}$ neurons consist of a heterogenous population of cells wherein some neurons express Ctip2 and project subcortically and others express Satb2 and project callosally. We have investigated the molecular mechanisms downstream of Satb2 and Ctip2 in deep layer neurons, which enables a population of cells born around the same developmental time point, occupying the same laminar position in the cortex, make opposite decisions with respect to their projections.

\section{$\underline{4.1}$ Commissural and sub-cortical projections are disrupted in}

\section{the Sip1 mutant}

Many different cortical axon tracts are severely altered in the absence of Sip1 (Figure 44). The CC, which forms the most important commissural projection in the brain, is absent in the Sip1 mutant. While the callosal neurons are able to project towards the cortical midline, these axons fail to cross the midline and form Probst bundles. The AC, which is an evolutionarily more primitive commissural projection in the brain is also absent in the Sip1 mutant. This makes the Sip1 mutant a very interesting model to study not only from a developmental point of view but also its behavioral aspects, as all forms of interhemispheric cortical projections are absent making each neocortex an independent unit. While the two commissural projections that originate from within the cortex are absent in the Sip1 mutant, the hippocampal commissure, 
which is a commissural tract that interconnects the two hippocampi is unaffected. This is an interesting finding as it provides evidence that Sip1 control over the formation of commissural projections is specific to cortical neurons and does not extend to hippocampal neurons, even though Sip1 is expressed in hippocampal neurons.

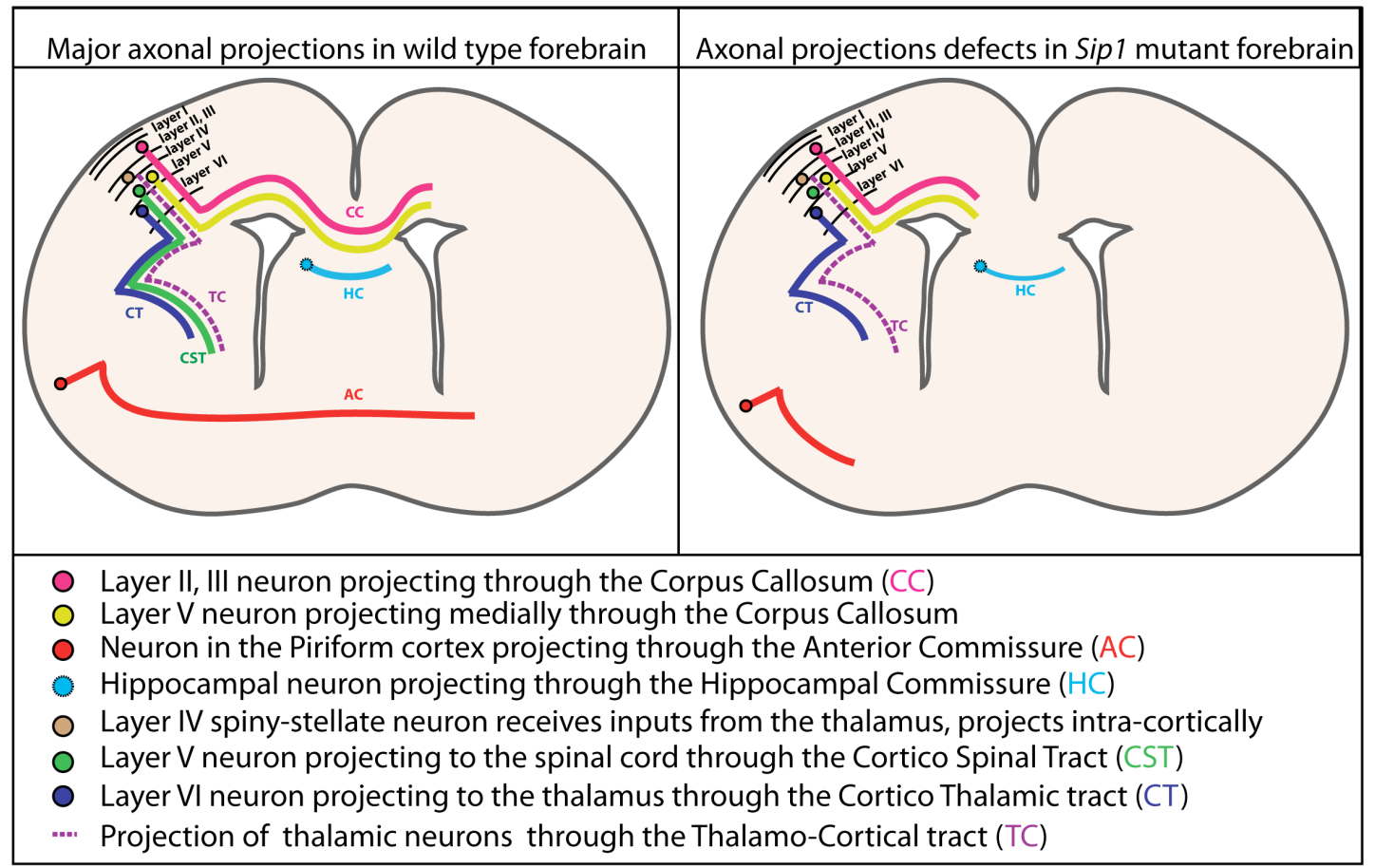

Figure 44. Connectivity defects observed in the Sip1 mutant forebrain

A summary of the connectivity defects observed in the Sip $1^{f / f l} \mathrm{Nex}$ Cre conditional mutant when compared to the wild type. The corpus callosum (CC), anterior commissure (AC) and corticospinal tract (CST) are absent. The cortico-thalamic tract (CT), thalamo-cortical tract (TC) and hippocampal commissure (HC) are unaffected by Sip1 deletion.

A possible explanation for the presence of the HC in the Sip1 mutant while the $\mathrm{CC}$ does not form could be because the $\mathrm{HC}$ forms further ventral to the CC. In the Sip1 mutant, since the fusion of the dorsal midline does not occur, CC axons cannot cross, but since midline fusion does occur ventrally, HC axons have the required scaffold to navigate across to the contralateral side. At the outset, this argument does not hold true for the $\mathrm{AC}$, since these axons traverse further ventral to the $\mathrm{HC}$ in the wild type forebrain, but do not form in the Sip1 mutant although midline fusion has occurred ventrally. DiI labeling at P0 in the Sip1 mutant brain showed that AC axons misproject 
ventrally from the external capsule instead of projecting towards the contralalteral hemisphere. We hence, believe that the absence of the AC axons in the Sip1 mutant is due to misprojection of the axons rather than midline defects.

Cortical connections projecting to subcortical targets are also affected to varying degrees in the Sip1 mutant (Figure 44). While the CT tract projecting to the thalamus is present in the Sip1 mutant, the CST is absent. Although initial projections of the CST can be seen at the level of the cerebral peduncle of Sip1 mutants at P0, these projections disappear by P5. This loss of CST in the Sip1 mutant at early postnatal stages is not due to cell death of layer $\mathrm{V}$ neurons as confirmed by cleaved caspase 3 staining in the neocortex at P2. This indicates that the loss of CST in the Sip1 mutant is due to axon guidance or axon growth defects.

It is interesting to note that of all the axonal tracts originating from within the neocortex it is only the CT tract that is unaffected in the Sip1 mutant. This might be correlated to the fact that the CT is the only tract, which depends on an external (non-cortical) source of axons to function as pioneer neurons (Koester \& O'Leary, 1994; McConnell et al., 1989; Molnar, Adams, \& Blakemore, 1998; Rash \& Richards, 2001). It is probable that like all other neocortical tracts the neocortical pioneer axons are also affected in the Sip1 mutant which in turn influence the formation of the commissural projections and the corticopsinal tract. In case of the CT, since the incoming thalamic axons are unaffected, the CT might be affected to a lesser degree. Interestingly, the CST, which follows the CT in its initial trajectory till the internal capsule, is also present in the Sip1 mutant at early stages. Its disappearance after P0 might be due to the absence of guidance cues further ahead in the trajectory or deficiency in internal axonal machinery of the neuron.

An alternate possibility for the presence of CT but none of the other cortical tracts could be that, the trajectory of corticothalamic axons does not cross the 
midline. While the CC crosses the midline at the dorsal forebrain and AC at the ventral forebrain, the CST crosses the midline at the pyramidal deccussation in the brainstem. As the midline is a very important intermediate target that involves a complex interplay between attractive and repulsive cues; specific receptors need to be expressed by the crossing axons at specific time points in order to cross the midline (Chen et al., 2008; Colamarino \& Tessier-Lavigne, 1995; Dickson \& Zou, 2010; Evans \& Bashaw, 2010; Kaprielian et al., 2000; Kidd et al., 1998; Klämbt, Jacobs, \& Goodman, 1991; Long et al., 2004), which in turn may be under Sip1 control. For example, Robo-Slit interactions have been shown to be extremely important for midline crossing. Our in situ hybridization experiments in the Sip1 mutant show that Robo2 is severly downregulated in the absence of Sip1.

\subsection{Dual role of Sip1 in CC formation}

Heterozygous mutation in SIP1 gene cause a disease known as the MowatWilson syndrome in humans which is characterized by severe craniofacial defects, microcephaly and severe mental retardation. In most cases, it is also associated with agenesis of the CC (Cacheux et al., 2001; D R Mowat, M J Wilson, 2003; Garavelli \& Mainardi, 2007; Moal et al., 2007; Verstappen et al., 2008; Wakamatsu et al., 2001; Wilson et al., 2003; K. Yamada et al., 2001), which makes studying the role of Sip1 in CC formation essential, also from a clinical point of view. We firstly dissected out the cell autonomous as well as the non-cell autonomous function of Sip1 in aiding callosum formation. Reexpression of Sip1 in the Sip1 mutant neocortex, so as to express the molecule in a mosaic manner in a mutant background, showed that unlike Sip1 deficient neurons that stall within the ipsilateral neocortex much before the midline fissure, the Sip1 over-expressing neurons were capable of extending their axons all the way till the midline fissure and stopped short of crossing the midline. This experiment goes to show that Sip1 plays a dual role with respect to CC formation. It cell intrinsically controls certain factors, at least within callosal neurons, that help in extension of the axons towards the 
midline, and additionally, the lack of midline crossing shows that there are cell extrinsic mechanisms involved as well.

\section{$\underline{4.3 \text { Non-cell autonomous role of Sip1 at the cortical midline }}$}

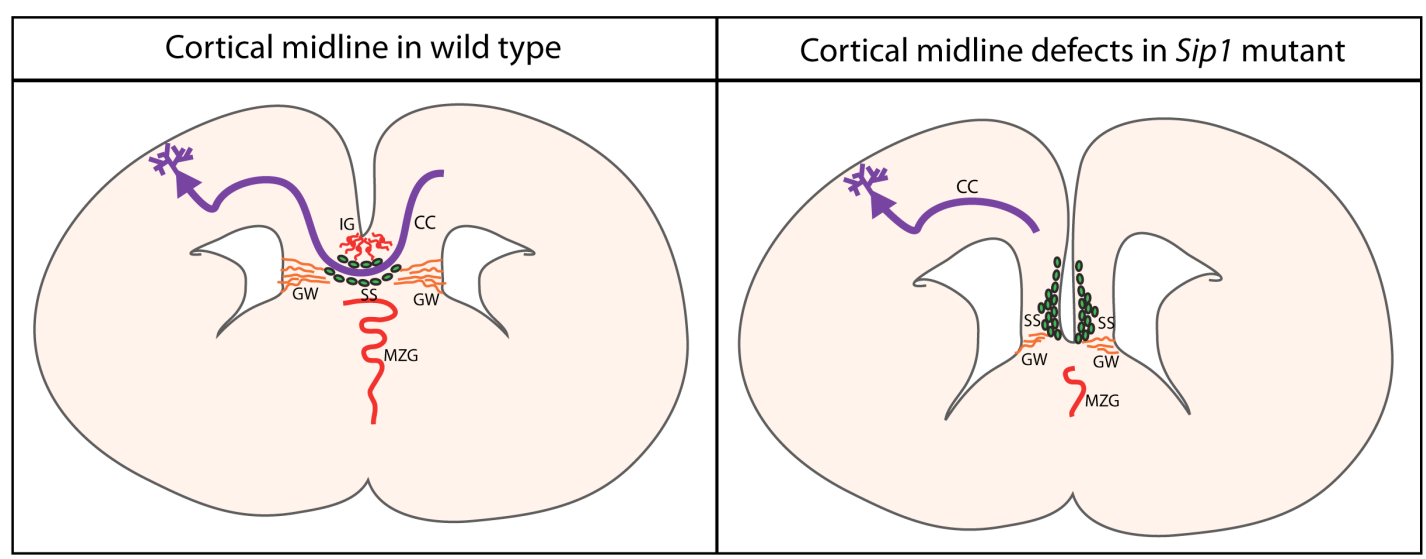

Figure 45. Non-cell autonomous defects in the Sip1 mutant midline

A schematic representation of the wild type and Sip $1^{f / f l} \mathrm{Nex}{ }^{\mathrm{Cre}}$ mutant midline, summarizing the non-cell autonomous effects of Sip1 deletion on the morphology of the cortical midline and its effect on callosal projections. In the Sip1 mutant the dorsal midline does not fuse, no indusium griseum glia (IG) is present, glial wedge (GW) and midline zipper glia (MZG) are highly reduced, sub callosal sling neurons (SS) are mislocalized. As a consequence callosal axons (CC) do not pass the mutant midline and instead form Probst bundles.

The midline forms a very critical intermediate guidepost structure for the formation of commissural projections. In case of the $\mathrm{CC}$, the cortical midline possesses various physical and chemical cues, which act as short and long range signals to help direct axons towards the contralateral hemisphere (Niquille et al., 2009; Shu \& Richards, 2001; Shu et al., 2003; Shu et al., 2003; Silver et al., 1993). In the Sip1 mutant, the cortical midline shows a variety of developmental defects (Figure 45). Firstly, unlike in the wild type neocortex, the dorsal midline in the Sip1 mutant is not fused, thus not providing a pathway for axons to pass through. The process of midline fusion is thought to be performed by midline zipper glia (MZG) that replace the fibroblasts present in the midline fissure with CNS tissue (Richards, Plachez, \& Ren, 2004; Shu \& Richards, 2001; Silver et al., 1993). The MZG is severely reduced in the Sip1 mutant. Secondly, midline glia that act as guidepost cells namely the indusium griseum cells (IG) and the glial wedge cells (GW) are not 
formed in the Sip1 mutant. These structures express Slit family of proteins, which provide a repulsive environment for Robo expressing callosal neurons (Shu \& Richards, 2001). Concurrent with the loss of midline glia, Slit1 expression is also lost in the Sip1 mutant midline. Since Sip1 expression is restricted to the post-mitotic population of the neocortex, the lack of midline glial cells is probably not due to a proliferation defect at the midline, but most-likely due to defects in the differentiation process of the radial glia into the midline glia. Thirdly, sub-callosal sling neurons (SS) that form another group of guidance structures for the formation of the CC (Niquille et al., 2009; Shu et al., 2003), are also absent in the Sip1 mutant. They also do not express Sema3C, which acts as an attractive guidance cue for the Nrp1 expressing pioneer axons (Niquille et al., 2009). The contribution of Sip1 to the formation of these midline guidance structures is a non-cell autonomous effect, as deleting Sip1 by Cre electroporation in a small population of cells within the cortical midline of Sip1 $1^{f / f l}$ embryos does not result in a phenotype similar to the Sip $1^{f l f l}$ Nex ${ }^{C r e}$ mutant.

It has not been clearly understood whether the formation of sling neurons is independent of the formation of the CC and precedes the crossing of the first callosal fibers or if the pioneering fibers of the CC assist in the migration of these sling neurons hence exhibiting a more synergistic interdependent relationship. The Sip1 mutant can be used as an interesting model for studying such a relationship. For example, since re-expression of Sip1 in the mutant neocortex causes further extension of the callosal fibers at the midline, using live imaging experiments, one could follow the difference in behavior of the sling neurons between a mutant scenario and Sip1 re-expression scenario. This would help to shed further light on the accessory role of the cortical midline in assisting CC formation. 


\section{$\underline{4.4 \text { Cell autonomous regulation of axon development by Sip1 }}$}

We observed that on cell autonomous deletion of Sip1 within a subset of cortical projection neurons, callosal fibers were unable to cross the midline by P0. While at E18.5 these axons did not make it to the contralateral hemisphere, by P8 at least most of the fibers could cross over. This together with the in vitro axon length assay showed that Sip1 deletion results in shortened axons due to a stunted growth rate. Additionally, cell intrinsic deletion of Sip1 in UL neurons, also affects the formation of interstitial axonal collaterals, which help in establishing synaptic contacts with the dendrites of Layer V pyramidal neurons. These findings hence suggest that apart from the non-cell autonomous role of Sip1, that regulates the architecture and cell specification of the cortical midline, Sip1 controls many cell intrinsic aspects in cortical neurons that are important for axon growth, guidance and branching, making it an essential transcription factor for the development of neocortical circuitry.

\subsection{Mechanism involved in cell intrinsic control of Sip1 over axon growth and guidance}

The formation of axonal projections that connect specific neurons to specific targets is a multi-step process that involves a plethora of axon guidance molecules that help the axonal growth cone navigate through a permissive environment while avoiding repulsive cues (Tessier-Lavigne \& Goodman, 1996). While one form of molecular control over axon guidance regulates the time and expression pattern of guidance cues, regulation of various cytoskeletal structures that are at the core of growth cone behavior in response to these extracellular cues, is another critical step (Conde \& Cáceres, 2009; Erik W Dent et al., 2011; Erik W. Dent, Tang, \& Kalil, 2003; Quinn \& Wadsworth, 2008). Our findings show that Sip1 exerts a regulatory role on both these critical aspects of axon guidance - regulating the expression of axon guidance cues as well as influencing cytoskeletal modifications. 
The expression of many different axon guidance molecules expressed by cortical projection neurons like Cntn2, EphrinA4, Robo2 and Nrp2 were affected in the Sip1 mutant. While it remains to be investigated if Sip1 regulates the expression of these molecules directly or indirectly, it can indeed be inferred that alterations in these many important guidance signals could be contributing to the vast number of guidance defects in the Sip1 mutant.

Loss of Sip1 in the neocortex also leads to a severe downregulation of ninein, a minus-end MT binding protein. In addition, as confirmed by ChIP assay, Sip1 directly regulates the transcription of ninein by binding to its promoter region (Figure 46A). Although in vivo Sip1 acts as a transcriptional activator of ninein, when luciferase assay was performed in vitro in HEK293T cells, there was a significant downregulation of transcription driven by the ninein enhancer region in the presence of Sip1. This discrepancy between the in vivo and in vitro function could probably be attributed to the fact that a heterologous system was used for the luciferase assay, and probable cofactors that might be required for Sip1 to behave as an activator were absent like activated RSMADs and SMAD4 (Yoshimoto et al., 2005). In the heterologous system used for the luciferase assay, such co-factors might not be present. It is also possible that Sip1 activation of ninein is specific to neurons as it might be binding to neuron specific co-factors that aid in the transcriptional activation of ninein. The role of Sip1 as a transcriptional repressor has been well established (Comijn et al., 2001; Postigo \& Dean, 2000; Postigo et al., 2003; Postigo, 2003; Remacle et al., 1999; Verschueren, 1999), with few well-characterized instances where Sip1 has been shown to play the role of an activator (Yoshimoto et al., 2005). The direct interaction between Sip1 and ninein enhancer in this study further strengthens the evidence that Sip1 can not only behave as a repressor but also as an activator and can thus perform a broader regulatory role depending on the cellular context. 


\subsection{Ninein localization and function}

Ninein was initially discovered to be a centrosome-associated protein localized to the pericentriolar matrix of cells that possess a radial array of MTs. In these cells, ninein acts as a MT minus-end binding protein that helps to anchor the MTs to the centrosome (Bouckson-Castaing et al., 1996). In cells that are polarized and show the presence of non-centrosomal MTs, ninein is also shown to be present in non-centrosomal locations in association with the MT minus-end, thus conferring a more general 'MT minus-end capping' role to ninein that is independent of the centrosome (Mogensen et al., 2000). Additionally Moss et al., have shown that ninein granules are transported in a MT dependent manner from the centrosome to peripheral locations supporting a non-centrosomal role for ninein (Moss et al., 2007).

In neurons, the presence of ninein has previously been shown in the somatodendritic compartments of the cell (Baird et al., 2004; Ohama \& Hayashi, 2009). In this study we have presented evidence using direct immunohistochemical labeling and expression of GFP tagged ninein, that ninein is also present in the axonal compartment of neurons (Figure 46B). In vivo, ninein is expressed in the white matter of the neocortex that corresponds to the fasciculate axons of pyramidal projection neurons. In vitro, in cultured cortical neurons at DIV 2 and DIV 7, GFP-ninein could be observed in the somato-dendritic as well as axonal compartments of the cell including the axonal collaterals. These results hence suggest that ninein could be playing a more extensive role in neurons that is not restricted to the somato-dendritic compartments.

Overexpression of ninein in Sip1 deficient neurons could rescue the axon extension defect both in vitro and in vivo. In vivo, although the axons of the electroporated neurons could extend across the midline into the contralateral hemisphere, the extent of midline crossing and invasion into the contralateral hemisphere was lesser than in the wild type. One possible explanation for such a partial rescue of the CC could be attributed to the fact that many axon 
guidance molecules also showed altered expression patterns and levels in the Sip1 mutant. These axon guidance molecules, apart from dictating the course of the axonal trajectory also act as signals that initiate axonal outgrowth and regulate the rate of axon extension (Quinn \& Wadsworth, 2008).

\subsection{Mode of action: Ninein stabilizes microtubules}

Neurons are extremely polarized cells and the bulk of MTs in neurons are present as free MTs in axons and dendrites (Ahmad \& Baas, 1995; Keating et al., 1997). Presence of non-centrosomal MT arrays allows for a more diverse set of MT arrangement and hence a diverse set of cellular morphology. It has been proposed that although the free MTs are not attached to the centrosome at the minus end, in order to remain stable, these MTs bind to other minus end stabilizing molecules (Keating \& Borisy, 1999; Murphy, Vallee, \& Borisy, 1977; Stearns \& Kirschner, 1994; Zheng, Wong, Alberts, \& Mitchison, 1995). In the absence of such a minus end stabilization, free MTs can form but do not persist (Keating \& Borisy, 1999). Experiments by Rodinov et al., have shown that in the absence of minus end capping, the minus end of MTs are very unstable and continually shorten. Hence regulating the levels and distribution of these minus-end capping/stabilizing proteins acts as a way to control MT distribution and turnover (Rodionov, Nadezhdina, \& Borisy, 1999).

The rescue of axonal length of Sip1 deficient neurons on re-expression of ninein together with the finding that that ninein is expressed in all cellular compartments, in addition to the previous knowledge that ninein binds MT minus-ends, makes it a very suitable candidate for a minus end capping and MT stabilizing protein. Evidence from previously published work has shown that in cells with radial MT arrays (where only centrosomal MTs are present), MTs associated with the ninein expressing mother centriole, are more resistant to MT destabilizing agents (Mogensen et al., 2000) and the knockdown of ninein from the pericentriolar matrix of such cells results in severe MT disorganization (Dammermann \& Merdes, 2002). Although these 
findings indicate that centrosome-associated ninein plays an important role as a stabilizing molecule for the radial MT asters, the role of non-centrosomal ninein has not been investigated so far. To our knowledge we have for the first time, shown that ninein plays a similar MT stabilizing role in neurons and possibly in other cells exhibiting non-centrosomal MTs as well (Figure 46C). Multiple pieces of evidence support this idea. Firstly, most cell types where non-centrosomal MTs have been studied, non-centrosomal ninein has also been found (Mogensen et al., 2000; Ohama \& Hayashi, 2009). Secondly, work by Baird et al., showed that in migrating cells where non-centrosomal MTs are required, non-centrosomal ninein might be responsible for capturing the minus end of released MTs so as to prevent re-capture by the centrosome (Baird et al., 2004). Thirdly, in the present study we have shown that in neurons, ninein localizes to all cellular compartments where MTs are present including axons and axonal branches. And most importantly, we have also shown, for the first time that non-centrosomal ninein can also act as a MTstabilizing agent in the axons and most likely dendrites of neurons. Neurons in culture over-expressing ninein show a very strong resistance to MT depolymerization in the axonal as well as dendritic compartments in the presence of Nocodazole- a microtubule destabilizing agent (De Brabander et al., 1976; Friedman \& Platzer, 1978; Hoebeke et al., 1976; Ireland et al., 1979; Lee et al., 1980).

\subsection{Ninein function in axon collateral formation}

During growth and navigation of axons, MTs reorganize and re-orient themselves towards the direction of axon growth. Similarly at the vicinity of the target region of an axon from which interstitial branches emerge, MTs undergo extensive re-organization (Dent et al., 1999; Dent et al., 2003; Gallo, 2011; Kalil et al., 2000; Schmidt \& Rathjen, 2010). Overexpression of ninein in Sip1 deficient upper layer neurons could also rescue the axonal collaterals formed by these axons at layer V. Similar mechanism might be involved downstream of Sip1 in axon growth and branch initiation, mediated by 
ninein. For example, just as MTs are severed and transported into the growing axon from the centrosome with the help of enzymes like katanin and spastin (Ahmad et al., 1999; Baas, 1998; Sharp \& Ross, 2012), at the interstitial branch sites too, MTs are cleaved by these enzymes (Yu et al., 1994; Yu et al., 2008). This generates smaller MTs, while simultaneously generating many free minus ends that need to be stabilized. We propose that similar to its role in the main axonal shaft, ninein might be required to re-capture the free MT minus-ends generated at the interstitial branch points to stabilize the newly formed MTs. A second possibility is that the defect in axon branch formation in the Sip1 mutant is secondary to the retarded axon growth. For example, target-derived signals from the contralateral neocortex might be required for callosal upper layer neurons to start forming collaterals at the ipsilateral layer V. Since Sip1 deficient axons are much slower than their wild type counterparts, they show a delay in reaching the target and in turn a delay in branch formation. In the presence of ninein, since the rate of extension of the axon is partially restored, the branching process is also rescued. Further experiments where the GFP-IRES-Cre electroporated Sip1flfl brains are taken at sequential stages beyond P7 will help to deduce if collateral development in the Sip1 deficient scenario is completely abolished or is only retarded similar to the axon growth defect.

\subsection{Microtubule stability influencing microtubule growth}

MTs exist in a state of dynamic instability, where the macromolecules oscillate between a state of growth or polymerization and break down or catastrophe, which may or may not be interspersed with a pause period (Mitchison \& Kirschner, 1984a, 1984b; Walker et al., 1988). This dynamic instability enables rapid changes in the cytoskeleton that in turn influences axon growth, guidance and branching (Conde \& Cáceres, 2009). Since MTs form the basic internal architecture of any cell, the growth rate of the MTs dictates the growth rate of the axon (Daniels, 1973; K. M. Yamada, Spooner, \& Wessells, 1970). As the growth rate of MTs at the plus-end is much faster than the 
minus-end, MT plus-end dynamics serves as a good indicator of net MT growth rate (Akhmanova \& Steinmetz, 2008). Several MT binding proteins have been shown to bind specifically to the plus-end, thus making it possible to track MT dynamics by tracking the movement of these plus-end associated molecules (Galjart, 2005). One such molecule that has been successfully used for studying MT growth is EB3 (Stepanova et al. 2003). Examining GFP tagged EB3 dynamics in Sip1 deficient neurons revealed that the plus end growth of the MTs was significantly slower when compared to controls, which also co-related with the finding that Sip1 negative neurons have slow growing axons.

Many different factors control and influence the rate of MT growth (Cassirneris, 1993; Erickson \& Brien, 1992), one such factor being the presence of stabilizing or destabilizing molecules associated with MTs. For example, in the presence of nanomolar concentration of Nocodazole, a MT destabilizing agent, the dynamic instability of MTs is disrupted and the MTs show very slow growth rate at the plus-end (Vasquez et al., 1997). Since ninein overexpressing neurons showed resistance to nocodazole treatment indicating a more stable MT framework in addition to the finding that the rate of axonal growth in Sip1 mutants could be rescued by ninein, we hypothesized that ninein might contribute to the MT growth in these cells (Figure 46C). When GFP-EB3 dynamics was tested, we observed the rate of growth of MTs returned to wild type levels on expressing ninein in Sip1 negative cells, hence indicating that ninein influences the dynamic instability of MTs thereby regulating axonal growth. It is still unclear as to how ninein being a MT minus-end binding protein could influence plus-end dynamics. We suggest two possibilities. By binding to the MT, ninein serves to primarily stabilize the growing MT polymer to which new tubulin dimmers are added, and in the absence of ninein, this MT lattice might be weak hence reducing the rate at which polymerization takes place. Alternatively, ninein might have additional role in MT dynamics other than binding to the minus-end. For example, in dendritic growth cones it has been shown that about $40 \%$ of 
ninein granules observed were not associated with MTs (Ohama \& Hayashi, 2009). These free ninein molecules might intermittently associate with other MAPs or regions other than the MT minus-end and in turn directly influence the plus-end dynamics. Such a dual role for a minus-end binding protein to directly regulate the dynamics of the plus end has been shown in case of the $\gamma$-TurC complex in Drosophila S2-cells (Bouissou et al., 2009).

\subsection{New insights gained: The role of Sip1 in axon development}

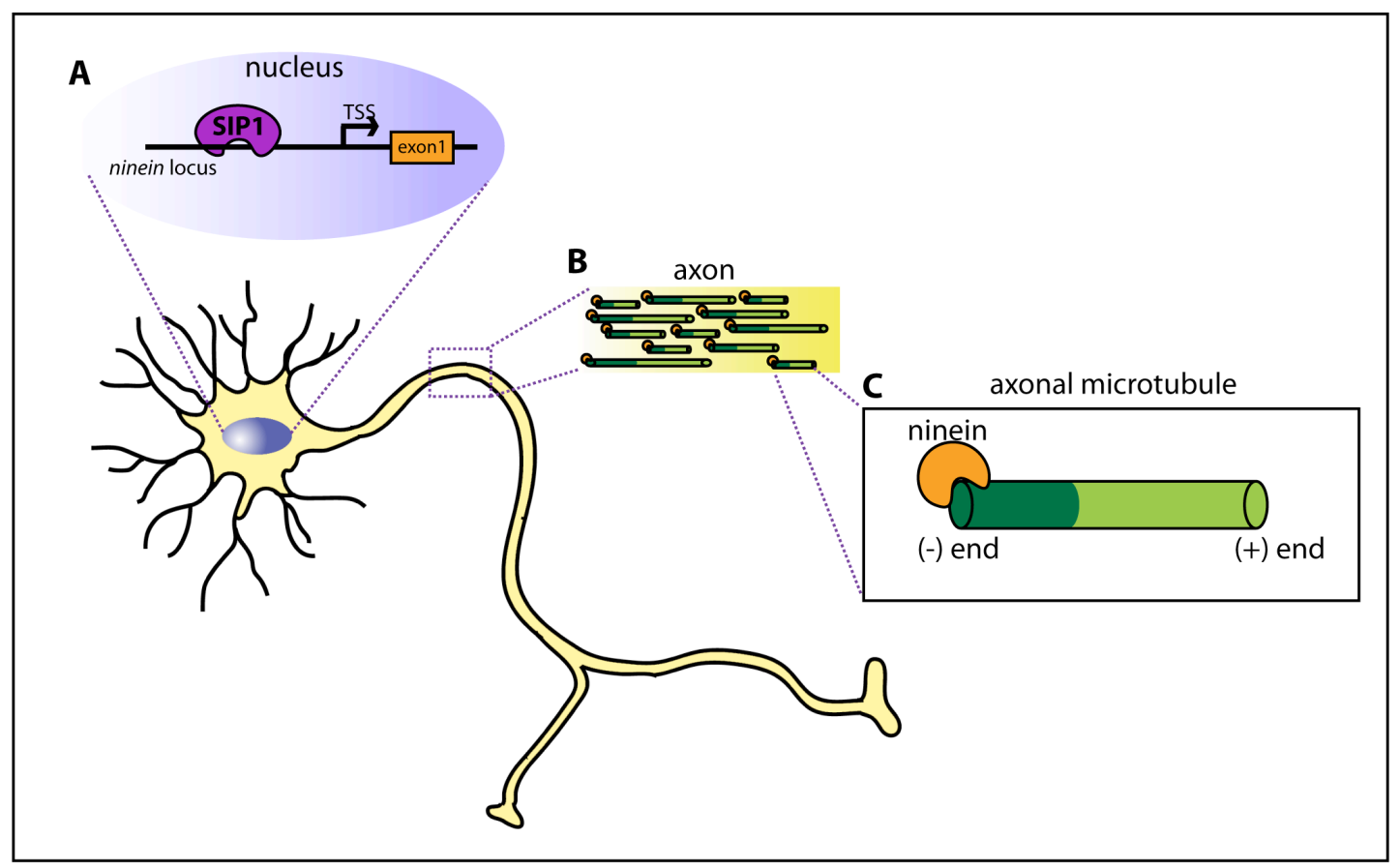

Figure 46. Ninein localizes to neocortical axons and influences microtubule growth and stability downstream of Sip1

The schematic representation summarizes the cell autonomous role of Sip1 in axon growth and branching. (A) Transcriptional factor Sip1 binds to the ninein promoter and activates its expression in cortical postmitotic neurons. (B) Ninein protein localizes to the minus end of the non-centrosomal MTs in the soma, dendrites, axon and axonal branches. (C) Ninein influences the rate of axon growth and the formation of axonal branches by stabilizing MTs and influencing MT growth rate.

In this study, we provide evidence to show that Sip1 transcriptionally activates the expression of ninein. Ninein binds to the minus end of free MTs and is present in the soma dendrites as well as the axons of neurons. By stabilizing the MT and regulating the growth of MTs, ninein influences axonal growth as well as branch formation. Although, the work presented here 
concentrates on the formation of the $\mathrm{CC}$, it is quite probable that such a Sip1 ninein dependent mechanism for axon growth is not specific to callosal neurons but extends to other cortical neurons as well. This idea is supported by the findings that in the Sip1 mutant almost all cortical projections are affected. Additionally, ninein does not show a layer specific pattern of expression in the wild type cerebral cortex. On these lines we could speculate that the loss of CST in the Sip1 mutant could be due to slow growing axons that do not reach a specific intermediate target at an appropriate developmental time point. It is hence withdrawn and does not exist at later developmental stages. Further experiments, specifically looking at the formation of other cortical tracts in the Sip1 mutant need to be performed in order to understand the role of ninein in these cells. 


\subsection{Satb2 and Ctip2 control the formation of divergent axonal}

\section{paths}

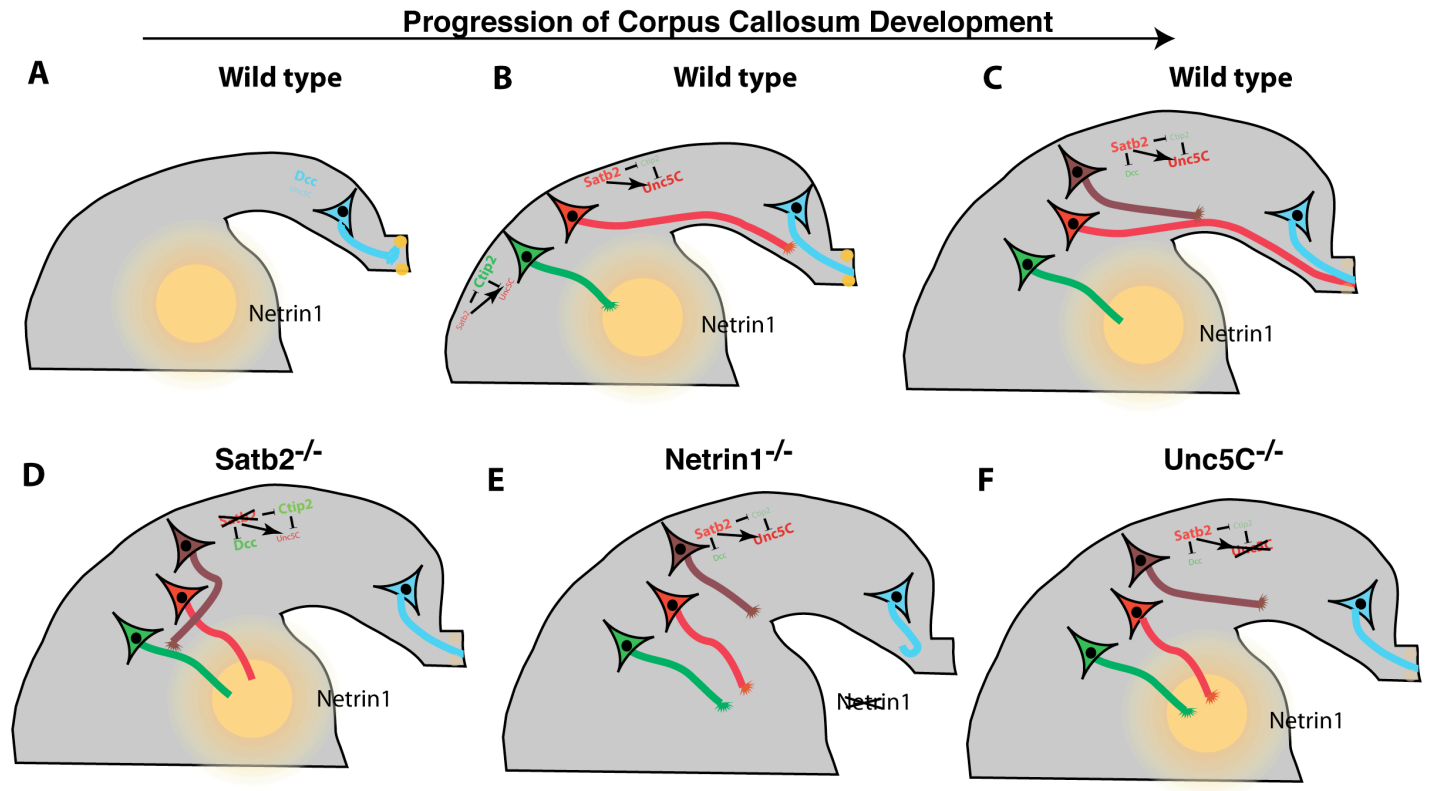

Figure 47. Satb2 Ctip2 dependent establishment of cortical connections

(A) During early corticogenesis cingulate neurons with high DCC expression form the pioneer axons for the CC. These axons respond to midline Netrin1 and are attracted towards the Netrin1 source (B) Later, when Layer V cells start projecting medially, Satb2 represses Ctip2, thereby promoting Unc5C expression. These Unc5C positive axons are repelled by the Netrin1 source in the internal capsule and thus turn towards the midline. Corticofugally projecting neurons however, express high levels of Ctip2 and thus repress Unc5C. Additionally the lack of repression of DCC by Satb2, promotes high DCC levels in these neurons. Thus, these axons are attracted towards the Internal capsule (C). Despite expressing high levels of Unc5C and low levels of DCC, upper layer neurons are not dependent on these molecules and instead either follow the deep layer callosal pioneer axons or are dependent on other axon guidance molecules. (D-F) Represent the scenario in the Satb2, Netrin1 or Unc5C mutants, respectively. In each of the mutants, the lack of an Unc5C-Netrin1 interaction causes a mis-routing of deep layer callosal axons to sub-cortical targets.

The expression of Satb2 or Ctip2 in cortical neurons confers mutually exclusive genetic programs with respect to their targeting (Alcamo et al., 2008; Arlotta et al., 2005; Britanova et al., 2008; Lickiss et al., 2012). Ctip2 dictates a corticofugal fate and is required for the fasciculation and appropriate targeting of these fibers (Arlotta et al., 2005). In contrast, deletion of Satb2 from the neocortex results in the absence of the CC and misrouting of callosal axons towards the internal capsule. Deletion of Satb2 also causes an ectopic up-regulation of Ctip2 in callosal neurons as Satb2 acts upstream of Ctip2 and represses it (Alcamo et al., 2008; Britanova et al., 2008). Although, a 
generalization can be made that Satb2 marks callosal neurons, it must be noted that not all callosal neurons express Satb2 (Britanova et al., 2008). The complete absence of the CC in Satb2 mutants suggests that callosal axons may require Satb2 both cell intrinsically and cell extrinsically. At the same time, some Satb2 positive neurons within the deep cortical layers, do not downregulate Ctip2, and project to the internal capsule (Britanova et al., 2008). Satb2 was shown to require the transcriptional co-factor Ski in order to repress Ctip2, therefore, these Satb2-positive/Ctip2-positive/Ski-negative cells are apparently non-callosal neurons (Baranek \& Atanasoski, 2012; Baranek et al., 2012).

\subsection{Lack of Ctip2 repression in the absence of $S a t b 2$ contributes to the acallosal phenotype in Satb2 $\%$ mice}

In this study we have shown that the ectopic up-regulation of Ctip2 in callosal neurons is one of the reason that contributes to the lack of a CC in Satb2\% mutants. Upon deletion of Ctip2 from the Satb2-\% cortex using Satb2- ;Ctip2\% double mutants, the CC was partially restored. Similar results were also recently reported (Alcamo et al., 2008; Britanova et al., 2008; Srinivasan et al., 2012). As the rescue was only partial, other factors in addition to the upregulation of Ctip2, could be responsible for the lack of a CC in Satb2\% mutants. Since the up-regulation of Ctip2 in Satb2\% mutants is a cell intrinsic effect, the rescue we observed in the Satb2-Ctip $2^{-\%}$ brains is likely to reveal the cell intrinsic ability of Satb2-positive cells to form the CC (Britanova et al., 2008).

\subsection{Unc5C is under the direct transcriptional control of Ctip2 and indirect control of Satb2}

Unc5C and DCC act as receptors for the secreted ligand Netrin1, mediating either a chemo-repulsive (Unc5C-DCC together) or a chemo-attractive (DCC alone) response (Colamarino \& Tessier-Lavigne, 1995; Fazeli et al., 1997; 
Hamelin et al., 1993; Hedgecock, Culotti, \& Hall, 1990). DCC independent role for Unc5C has also been shown. Expression patterns of both these receptor molecules in the developing neocortex are very dynamic. DCC is expressed at a high level in the early cortical plate at E14.5 and it is down regulated in later born neurons between E16.5-E18.5. In contrast, the highest levels of Unc5C expression are detected in later born neurons at E16.5-E18.5 and its expression is low in the early cortical plate. The expression of these two genes is also different in the neocortex of Satb2 mutants: while Unc5C is down-regulated in the absence of $S a t b 2, D C C$ is upregulated (Figure 47D).

Ctip2 acts as a transcriptional repressor of Unc5C. Multiple pieces of evidence support this finding. Firstly, Unc5C expression is regained in Satb2-/Ctip2-/compound mutants. Secondly, we demonstrated through ChIP and luciferase assay that Ctip2 is a direct transcriptional repressor of Unc5C. Thirdly, electroporation of Satb2 could ectopically induce Unc5C expression in cells when Ctip2 was downregulated. Thus, Satb2 controls the expression of Unc5C in the neocortex indirectly by repressing Ctip2 expression. Overexpression of Unc5C in Satb2\% mutants, similar to the Satb2- ;Ctip2\% double mutant, also leads to a partial restoration of the CC, demonstrating a cell autonomous ability for Unc5C to promote callosal targeting. This result was independently confirmed in a recent publication (Srinivasan et al., 2012).

\section{$\underline{4.14}$ Unc5C mutants also show corpus callosum defects}

It has been previously reported that Unc5C mutants show a normal CC, with cortical fibers crossing the midline (Ackerman et al., 1997). Contrarily, our experiments indicate that Unc5C contributes cell autonomously to the formation of the CC. The question then arises that if $U n c 5 C$ is required for $C C$ formation then why are there no obvious defects of the CC observed in Unc5C-- mutants? Retrograde labeling of sub-cortically projecting neurons in Unc5C $C^{-1}$ mutants showed that these mutants display an increase in the number of Satb2 positive neurons that project sub-cortically (Figure 47F). 
These misprojected axons that would have projected callosally in the wild type situation probably go undetected on histological and morphological analysis of the Unc5C null brains, as they constitute only a small percentage of the total axonal bundle. Thus, Unc5C is probably required for CC formation only in those neurons that rely on Satb2 cell intrinsically to project callosally. Since late electroporation of Unc5C does not rescue the callosum in Satb2\%mutants, these Unc5C dependent neurons seem to be early born deep layer neurons but not later born upper layer neurons.

\subsection{Unc5C aids in corpus callosum formation in response to Netrin1 expression}

Netrin1 belongs to a family of phylogenetically conserved proteins and acts as a long-range diffusible guidance cue (Hedgecock et al., 1990; Ishii, Wadsworth, Stern, Culotti, \& Hedgecock, 1992; Serafini et al., 1996; Wadsworth, Bhatt, \& Hedgecock, 1996). In the wild type forebrain, there are two major sources of Netrin1 in the developing telencephalon; one in the septum, at the cortical midline (in the vicinity of the CC) and the other in the basal ganglia (in the vicinity of the initial segment of the corticofugal tracts in the internal capsule) (Barallobre et al., 2000; Metin et al., 1997). With the help of an in vitro cortical slice culture model we showed that, Unc5C expressing cortical neurons were responsive to Netrin1 signals and were repelled by it. We further showed that similar to the Unc5C mutants, Netrin 1 mutants had a higher proportion of Satb2 positive cells projecting sub-cortically compared to wild type brains (Figure 47E). The differences in the proportion of Satb2 positive cells that we observed projecting subcortically in the Unc5C mutant when compared to Netrin1 mutants might be due to the different genetic backgrounds of these strains and/or because of the involvement of other Netrin1 receptors such as DCC and Draxin (Ahmed et al., 2011; Islam et al., 2009). Both these findings support the hypothesis that Unc5C mediated CC formation is dependent on Unc5C-Netrin1 repulsion. 


\subsection{Unc5C and Netrin1 mutants show similar axonal behavior} albeit dissimilar callosal phenotype

Although based on the theory put forward by this study, Unc5C and Netrin 1 control the same pathway for CC formation, an apparent discrepancy arises between the Netrin 1 mutant which is acallosal and the Unc5C mutant which is not. We propose that Netrin 1 mutant and the Unc5C mutant show a similar behavior with respect to cortical axons projecting medially. A probable explanation for this could be as follows. The Netrin1 mutants lack the expression of Netrin 1 at the basal ganglia as well as in the cortical midline. Midline Netrin 1 expression is important for CC formation as it is responsible for attracting DCC positive cingulate axons that pioneer the CC. In the Netrin1 mutant, since midline Netrin1 expression is lost, the pioneering axons of the CC do not cross (Thomas Fothergill et al., 2013)(Figure 47A). Although the Netrin 1 mutant does not show callosal crossing, the majority of callosal fibers nonetheless project medially and arrive at the midline. These fibers are unable to cross due to the absence of pioneering callosal axons forming Probst bundles instead (Ren et al., 2007). On the other hand, in Unc5C mutants, since the midline Netrin1 interaction with DCC-positive cingulate axons is not disrupted, callosal axons cross the midline. Therefore, we suggest that in Netrin $1 \%$ and Unc5C $\%$ mutant mice, only the callosal fibers that depend on Netrin1-Unc5C interaction misproject to sub-cortical targets, whereas neurons which depend on other signaling pathways, continue to project medially towards the midline. In the Unc5 $\mathrm{C}^{-1}$ brains they cross the midline but in the Netrin 1\% brains they stall at the midline.

It is further interesting to note that Unc5C expressing callosal neurons do not respond to midline Netrin1. This is probably because, at late embryonic stages (E16.5 onwards) the expression level of Netrin1 in the midline is very low as compared to the expression in the basal ganglia (Barallobre et al., 2000; Thomas Fothergill et al., 2013) and secondly, it has been shown that axons of later born cortical neurons do not require midline Netrin1 to form the CC (Thomas Fothergill et al., 2013) (Figure 47B). 


\subsection{Together with Netrin1 - Unc5C interaction, Netrin1 - DCC interaction regulates medial vs. lateral axonal trajectory choice}

In addition to the changes in Unc5C expression levels in the Satb2 mutant, we also observed that DCC was upregulated in the absence of Satb2 (Figure 47D). We demonstrated in vivo, through ChIP and in vitro luciferase assay that DCC is under direct transcriptional control of Satb2. We hence hypothesized that in the Satb2 mutant due to the upregulation of DCC in callosal neurons there might be an increase in DCC-Netrin1 attraction towards the internal capsule. Additionally, the downregulation of Unc5C results in reduced Unc5C-Netrin1 mediated repulsion. This hence forces callosal fibers, which are normally repelled by Netrin1 from the internal capsule in the wild type, to be attracted to the Netrin1 source in the Satb2 mutant. In support of our hypothesis, we found that down-regulation of DCC by electroporation of DCC shRNA in Satb2- mice, resulted in a partial rescue of the CC.

We hence propose that tempering down the DCC signal helps the axons make a medial choice and hence project callosally. At the outset, this theory seems to contradict the fact that DCC mutants display an acallosal phenotype (Fazeli et al., 1997). The question then arises that if the downregulation of DCC helps in CC formation, why does the DCC mutant show an acallosal phenotype. This apparent discrepancy could probably again be explained by the fact that the acallosal phenotype in the DCC mutant is characterized by callosal axons projecting medially but not crossing the midline (Fazeli et al., 1997). The lack of midline crossing is probably due to a secondary defect, the absence of DCC expression in the cingulate pioneer neurons (Figure 47A). Therefore, similar to the DCCshRNA electroporation in the Satb2 mutant, DCC mutant brains also show medial projections. A simultaneous co-electroporation of Unc5C together with DCC shRNA did not result in a more profound rescue of the CC. Instead we observed that there was no rescue at all. This is a surprising result indicating that the dosage of Unc5C/DCC in each cell might be critical in deciding the projection fate of cortical projection neurons (Muramatsu et al., 2010). 


\section{$\underline{4.18 \text { Satb2 } \%, \text { DCC } \% \text { and Netrin1 mutants show disparate accalosal }}$}

\section{phenotypes}

Although the Satb2 mutant, DCC mutant and the Netrin1 mutant are all acallosal, they differ with respect to the trajectory of these callosal fibers. While in both the Netrin1 and DCC mutants callosal fibers project medially but fail to cross the midline and form Probst bundles, in the Satb2 mutant, the acallosal phenotype is not restricted to the absence of callosal midline crossing, but occurs because of the absence of any neocortical axons navigating medially. Satb2 mutants do not display Probst bundles. The callosal axons are misrouted towards the internal capsule and the anterior commissure (Alcamo et al., 2008; Britanova et al., 2008). Overexpression of Unc5C or downregulation of DCC, in Satb2\% brains results in a subset of neurons projecting medially, which can be seen as a clear bundle of axons leaving the electroporated region and approaching the midline. Hence, it is not only the midline crossing of these axons that is of significance, but more so the choice to project interhemispherically (medially) and not in the corticofugal direction (laterally). Although we have not been able to detect any neocortical axons forming the CC in our Satb2 mutant, Alcamo et al. reported that their Satb2 mutant has a severely reduced callosum. These few fibers that remain in the Satb2 mutant could be attributed to the presence of DCC positive cingulate axons that project interhemispherically through the CC that maybe unaffected in the Satb2 mutant (Alcamo et al., 2008).

\subsection{Cell intrinsic control of Satb2/Ctip2 and hence DCC/Unc5C} in the formation of commissural vs. corticofugal projections is restricted to a sup-population of deep layer neurons

Our data strongly indicates that the control of Satb2 and Ctip2 over callosal and corticofugal fate specification is dependent on Unc5C and DCC only with respect to deep layer neurons (Figure 47B) as none of the rescue experiments performed at E14.5 lead to the formation of a CC. Secondly, the DiI 
experiments on Unc5C and Netrin1 mutants predominantly labeled deep layer neurons and thus the differences that we report here are most likely a reflection of the misrouting of deep layer Satb2 positive neurons. Electroporation of Satb2 into the Satb2\% brain could as well not rescue the entire acallosal phenotype in these mice, and was effective in rescuing only early born, deep layer, callosal projections. This clearly shows that the potential of each individual Satb2 positive cell to project callosally is highly limited and requires a supportive environment. Hence, it is likely that deep layer neurons act in a cell autonomous manner in deciding between callosal and sub-cortical fates and that later born Satb2 positive cells are dependent on other mechanisms for correct path-finding (Figure 47B, C).

\section{$\underline{4.20 ~ N e w ~ i n s i g h t s ~ g a i n e d ~: ~ U n d e r s t a n d i n g ~ t h e ~ m o l e c u l a r ~ b a s i s ~ o f ~}$ axonal trajectory choice}

Together the experiments performed in this study indicate an important role for Netrin1, DCC and Unc5C interaction in the trajectory choice made by deep layer cortical axons. We suggest a scenario where the source of Netrin1 in the internal capsule attracts Unc5C-negative/DCC-positive axons while repelling Unc5C-positive/DCC-negative axons. Hence, callosally projecting deep layer neurons require higher levels of Unc5C and lower levels of DCC while on the other hand, low levels of Unc5C and high levels of DCC instruct neurons to project subcortically (Figure 47A, B, C). It is still not clear how this Netrin1-DCC/Unc5C interaction mechanism functions during the initial steps of axon navigation. We suggest two alternative scenarios. One possibility is that cortical neurons send two axonal branches in two opposite directions, one towards CC and the other towards the internal capsule. The presence of such dual projections in early neocortical development has been reported previously (Garcez et al., 2007; Lickiss et al., 2012). Here, Unc5Cpositive/DCC-negative neurons would retract the lateral branch after arrival at the Netrin1 source in the internal capsule and stabilize the callosal branch. Unc5C-negative/DCC-positive neurons would retract the callosal branch and 
stabilize their subcortical branch. A second alternative scenario is that all early cortical neurons send their axons towards the internal capsule, but upon reaching the vicinity of a Netrin1 source, Unc5C-positive/DCC-negative neurons would retract these axons and develop an independent callosal branch. Further experiments using advanced live-imaging techniques can help answer these questions and shed further light on how the complex yet highly organized system of cortical connections is established. 


\section{REFERENCES}

Aboitiz, F., \& Montiel, J. (2003). One hundred million years of interhemispheric communication: the history of the corpus callosum. Brazilian Journal of Medical and Biological Research $=$ Revista Brasileira de Pesquisas Médicas E Biológicas / Sociedade Brasileira de Biofísica ... [et Al.], 36(4), 409-20.

Acebes, a, \& Ferrús, a. (2000). Cellular and molecular features of axon collaterals and dendrites. Trends in Neurosciences, 23(11), 557-65.

Ackerman, S. L., Kozak, L. P., Przyborski, S. A., Rund, L. A., Boyer, B. B., \& Knowles, B. B. (1997). The mouse rostral cerebellar malformation gene encodes an UNC-5-like protein. Nature, 386(6627), 838-842. doi:10.1038/386838a0

Ahmad, F. J., \& Baas, P. W. (1995). Microtubules released from the neuronal centrosome are transported into the axon. Journal of Cell Science, 108 ( Pt 8, 2761-9.

Ahmad, F. J., Yu, W., McNally, F. J., \& Baas, P. W. (1999). An essential role for katanin in severing microtubules in the neuron. The Journal of Cell Biology, 145, 305-315. doi:10.1083/jcb.145.2.305

Ahmed, G., Shinmyo, Y., Ohta, K., Islam, S. M., Hossain, M., Naser, I. B., ... Tanaka, H. (2011). Draxin inhibits axonal outgrowth through the netrin receptor DCC. J Neurosci, 31(39), 14018-14023. doi:10.1523/JNEUROSCI.0943-11.2011 31/39/14018 [pii]

Akhmanova, A., \& Steinmetz, M. O. (2008). Tracking the ends: a dynamic protein network controls the fate of microtubule tips. Nature Reviews. Molecular Cell Biology, 9(4), 309-22. doi:10.1038/nrm2369

Alcamo, E. A., Chirivella, L., Dautzenberg, M., Dobreva, G., Farinas, I., Grosschedl, R., \& McConnell, S. K. (2008). Satb2 regulates callosal projection neuron identity in the developing cerebral cortex. Neuron, 57(3), 364-377. doi:S0896-6273(07)01017-3 [pii] 10.1016/j.neuron.2007.12.012

Angevine, J. B., \& Sidman, R. L. (1961). Autoradiographic study of cell migration during histogenesis of cerebral cortex in the mouse. Nature, 192, 766-768. doi:10.1038/192766b0

Anthony, T. E., Klein, C., Fishell, G., \& Heintz, N. (2004). Radial glia serve as neuronal progenitors in all regions of the central nervous system. Neuron, 41, 881-890. doi:10.1016/S0896-6273(04)00140-0

Arlotta, P., Molyneaux, B. J., Chen, J., Inoue, J., Kominami, R., \& Macklis, J. D. (2005). Neuronal subtype-specific genes that control corticospinal motor neuron development in vivo. Neuron, 45(2), 207-221. doi:S0896627304008530 [pii] 10.1016/j.neuron.2004.12.036

Aronoff, R., Matyas, F., Mateo, C., Ciron, C., Schneider, B., \& Petersen, C. C. H. (2010). Long-range connectivity of mouse primary somatosensory barrel cortex. The European Journal of Neuroscience, 31(12), 2221-33. doi:10.1111/j.1460-9568.2010.07264.x 
Baas, P. W. (1998). The role of motor proteins in establishing the microtubule arrays of axons and dendrites. Journal of Chemical Neuroanatomy, 14, 175180. doi:10.1016/S0891-0618(98)00012-X

Baas, P. W., \& Ahmad, F. J. (1992). The plus ends of stable microtubules are the exclusive nucleating structures for microtubules in the axon. The Journal of Cell Biology, 116, 1231-1241. doi:10.1083/jcb.116.5.1231

Baas, P. I. V, \& Yu, W. (1996). A Composite Model for Establishing the Microtubule Arrays of the Neuron, 12(2), 145-161.

Bagnard, D., Lohrum, M., Uziel, D., Püschel, A. W., \& Bolz, J. (1998). Semaphorins act as attractive and repulsive guidance signals during the development of cortical projections. Development (Cambridge, England), 125, 5043-5053.

Baird, D. H., Myers, K. a, Mogensen, M., Moss, D., \& Baas, P. W. (2004). Distribution of the microtubule-related protein ninein in developing neurons. Neuropharmacology, 47(5), 677-83. doi:10.1016/j.neuropharm.2004.07.016

Bak, M., \& Fraser, S. E. (2003). Axon fasciculation and differences in midline kinetics between pioneer and follower axons within commissural fascicles. Development (Cambridge, England), 130(20), 4999-5008. doi:10.1242/dev.00713

Barallobre, M. J., Del Rio, J. A., Alcantara, S., Borrell, V., Aguado, F., Ruiz, M., ... Soriano, E. (2000). Aberrant development of hippocampal circuits and altered neural activity in netrin 1-deficient mice. Development, 127(22), 4797-4810.

Baranek, C., \& Atanasoski, S. (2012). Modulating epigenetic mechanisms: the diverse functions of Ski during cortical development. Epigenetics, 7(7), 676-679. doi:10.4161/epi.20590 20590 [pii]

Baranek, C., Dittrich, M., Parthasarathy, S., Bonnon, C. G., Britanova, O., Lanshakov, D., ... Atanasoski, S. (2012). Protooncogene Ski cooperates with the chromatin-remodeling factor Satb2 in specifying callosal neurons. Proc Natl Acad Sci U S A, 109(9), 3546-3551. doi:10.1073/pnas.1108718109 1108718109 [pii]

Bedogni, F., Hodge, R. D., Elsen, G. E., Nelson, B. R., Daza, R. A. M., Beyer, R. P., ... Hevner, R. F. (2010). Tbr1 regulates regional and laminar identity of postmitotic neurons in developing neocortex. Proceedings of the National Academy of Sciences of the United States of America, 107(29), 13129-34. doi:10.1073/pnas.1002285107

Bornens, M. (2002). Centrosome composition and microtubule anchoring mechanisms. Current Opinion in Cell Biology, 14(1), 25-34.

Bouckson-Castaing, V., Moudjou, M., Ferguson, D. J., Mucklow, S., Belkaid, Y., Milon, G., \& Crocker, P. R. (1996). Molecular characterisation of ninein, a new coiled-coil protein of the centrosome. Journal of Cell Science, 109 ( Pt 1, 179-90.

Bouissou, A., Vérollet, C., Sousa, A., Sampaio, P., Wright, M., Sunkel, C. E., ... Raynaud-Messina, B. (2009). \{gamma\}-Tubulin ring complexes regulate microtubule plus end dynamics. The Journal of Cell Biology, 187(3), 327-34. doi:10.1083/jcb.200905060 
Bray, D., \& Bunge, M. B. (1981). Serial analysis of microtubules in cultured rat sensory axons. Journal of Neurocytology, 10, 589-605. doi:10.1007/BF01262592

Britanova, O., de Juan Romero, C., Cheung, A., Kwan, K. Y., Schwark, M., Gyorgy, A., ... Tarabykin, V. (2008). Satb2 is a postmitotic determinant for upper-layer neuron specification in the neocortex. Neuron, 57(3), 378392. doi:S0896-6273(08)00033-0 [pii] 10.1016/j.neuron.2007.12.028

Britanova, O., Depew, M. J., Schwark, M., Thomas, B. L., Miletich, I., Sharpe, P., \& Tarabykin, V. (2006). Satb2 haploinsufficiency phenocopies 2q32q33 deletions, whereas loss suggests a fundamental role in the coordination of jaw development. Am J Hum Genet, 79(4), 668-678. doi:S0002-9297(07)63077-8 [pii] 10.1086/508214

Brose, K., \& Tessier-Lavigne, M. (2000). Slit proteins: key regulators of axon guidance, axonal branching, and cell migration. Current Opinion in Neurobiology, 10, 95-102. doi:10.1016/S0959-4388(99)00066-5

Burkhalter, A. (1989). Intrinsic connections of rat primary visual cortex: laminar organization of axonal projections. The Journal of Comparative Neurology, 279(2), 171-86. doi:10.1002/cne.902790202

Cacheux, V., Dastot-Le Moal, F., Kääriäinen, H., Bondurand, N., Rintala, R., Boissier, B., ... Goossens, M. (2001). Loss-of-function mutations in SIP1 Smad interacting protein 1 result in a syndromic Hirschsprung disease. Human Molecular Genetics, 10(14), 1503-10.

Cassirneris, L. (1993). Views and Reviews Regulation of Microtubule Dynamic Instability, 281, 275-281.

Chan, S. S., Zheng, H., Su, M. W., Wilk, R., Killeen, M. T., Hedgecock, E. M., \& Culotti, J. G. (1996a). UNC-40, a C. elegans homolog of DCC (Deleted in Colorectal Cancer), is required in motile cells responding to UNC-6 netrin cues. Cell, 87(2), 187-195. doi:S0092-8674(00)81337-9 [pii]

Chen, B., Schaevitz, L. R., \& McConnell, S. K. (2005). Fezl regulates the differentiation and axon targeting of layer 5 subcortical projection neurons in cerebral cortex. Proceedings of the National Academy of Sciences of the United States of America, 102(47), 17184-9. doi:10.1073/ pnas.0508732102

Chen, B., Wang, S. S., Hattox, A. M., Rayburn, H., Nelson, S. B., \& McConnell, S. K. (2008). The Fezf2-Ctip2 genetic pathway regulates the fate choice of subcortical projection neurons in the developing cerebral cortex. Proceedings of the National Academy of Sciences of the United States of America, 105(32), 11382-7. doi:10.1073/pnas.0804918105

Chen, Z., Gore, B. B., Long, H., Ma, L., \& Tessier-Lavigne, M. (2008). Alternative splicing of the Robo3 axon guidance receptor governs the midline switch from attraction to repulsion. Neuron, 58(3), 325-32. doi:10.1016/j.neuron.2008.02.016

Colamarino, S. A., \& Tessier-Lavigne, M. (1995a). The axonal chemoattractant netrin-1 is also a chemorepellent for trochlear motor axons. Cell, 81(4), 621-629. doi:0092-8674(95)90083-7 [pii]

Colón-Ramos, D. A., \& Shen, K. (2008). Cellular Conductors: Glial Cells as Guideposts during Neural Circuit Development. PLoS Biology, 6, 3. doi:10.1371/journal.pbio.0060112 
Comijn, J., Berx, G., Vermassen, P., Verschueren, K., van Grunsven, L., Bruyneel, E., ... van Roy, F. (2001). The Two-Handed E Box Binding Zinc Finger Protein SIP1 Downregulates E-Cadherin and Induces Invasion. Molecular Cell, 7(6), 1267-1278.

Conde, C., \& Cáceres, A. (2009). Microtubule assembly, organization and dynamics in axons and dendrites. Nature Reviews. Neuroscience, 10(5), 319-32. doi:10.1038/nrn2631

D R Mowat, M J Wilson, M. G. (2003). Mowat-Wilson syndrome, (Table 1), 305-311.

Dammermann, A., \& Merdes, A. (2002). Assembly of centrosomal proteins and microtubule organization depends on PCM-1. The Journal of Cell Biology, 159(2), 255-66. doi:10.1083/jcb.200204023

Daniels, M. P. (1973). FINE STRUCTURAL CHANGES IN NEURONS AND NERVE FIBERS ASSOCIATED WITH COLCHICINE INHIBITION. The Journal of Cell Biology, 58, 463-470.

De Brabander, M. J., Van de Veire, R. M., Aerts, F. E., Borgers, M., \& Janssen, P. A. (1976). The effects of methyl (5-(2-thienylcarbonyl)-1Hbenzimidazol-2-yl) carbamate, (R 17934; NSC 238159), a new synthetic antitumoral drug interfering with microtubules, on mammalian cells cultured in vitro. Cancer Research, 36(3), 905-16.

deAzevedo, L. C., Hedin-Pereira, C., \& Lent, R. (1997). Callosal neurons in the cingulate cortical plate and subplate of human fetuses. The Journal of Comparative Neurology, 386(1), 60-70.

Deiner, M. S., Kennedy, T. E., Fazeli, A., Serafini, T., Tessier-Lavigne, M., \& Sretavan, D. W. (1997). Netrin-1 and DCC mediate axon guidance locally at the optic disc: loss of function leads to optic nerve hypoplasia. Neuron, 19(3), 575-589. doi:S0896-6273(00)80373-6 [pii]

Delgehyr, N., Sillibourne, J., \& Bornens, M. (2005). Microtubule nucleation and anchoring at the centrosome are independent processes linked by ninein function. Journal of Cell Science, 118(Pt 8), 1565-75. doi:10.1242/jcs.02302

Dent, E. W., Callaway, J. L., Szebenyi, G., Baas, P. W., \& Kalil, K. (1999). Reorganization and movement of microtubules in axonal growth cones and developing interstitial branches. The Journal of Neuroscience : The Official Journal of the Society for Neuroscience, 19, 8894-8908.

Dent, E. W., Gupton, S. L., \& Gertler, F. B. (2011). The growth cone cytoskeleton in axon outgrowth and guidance. Cold Spring Harbor Perspectives in Biology, 3(3). doi:10.1101/cshperspect.a001800

Dent, E. W., Tang, F., \& Kalil, K. (2003). Axon Guidance by Growth Cones and Branches: Common Cytoskeletal and Signaling Mechanisms. The Neuroscientist, 9(5), 343-353. doi:10.1177/1073858403252683

Desai, A. R., \& McConnell, S. K. (2000). Progressive restriction in fate potential by neural progenitors during cerebral cortical development. Development (Cambridge, England), 127(13), 2863-2872.

Dickson, B. J., \& Zou, Y. (2010). Navigating Intermediate Targets : The Nervous System Midline Navigating Intermediate Targets : The Nervous System Midline. 
Eisaki, A., Kuroda, H., Fukui, A., \& Asashima, M. (2000). XSIP1, a member of two-handed zinc finger proteins, induced anterior neural markers in Xenopus laevis animal cap. Biochemical and Biophysical Research Communications, 271, 151-157. doi:10.1006/bbrc.2000.2545

Erickson, H. P., \& Brien, E. T. O. (1992). MICROTUBULE DYNAMIC INSTABILITY AND GTP HYDROLYSIS, 145-166.

Evans, T. a, \& Bashaw, G. J. (2010). Axon guidance at the midline: of mice and flies. Current Opinion in Neurobiology, 20(1), 79-85. doi:10.1016/j.conb.2009.12.006

Fame, R. M., MacDonald, J. L., \& Macklis, J. D. (2011). Development, specification, and diversity of callosal projection neurons. Trends in Neurosciences, 34(1), 41-50. doi:10.1016/j.tins.2010.10.002

Fazeli, A., Dickinson, S. L., Hermiston, M. L., Tighe, R. V, Steen, R. G., Small, C. G., ... Weinberg, R. A. (1997). Phenotype of mice lacking functional Deleted in colorectal cancer (Dcc) gene. Nature, 386(6627), 796-804. doi:10.1038/386796a0

Finger, J. H., Bronson, R. T., Harris, B., Johnson, K., Przyborski, S. A., \& Ackerman, S. L. (2002). The netrin 1 receptors Unc5h3 and Dcc are necessary at multiple choice points for the guidance of corticospinal tract axons. J Neurosci, 22(23), 10346-10356. doi:22/23/10346 [pii]

Fishell, G., \& Hanashima, C. (2008a). Pyramidal neurons grow up and change their mind. Neuron, 57(3), 333-338. doi:10.1016/j.neuron.2008.01.018

Fishell, G., \& Kriegstein, A. R. (2003). Neurons from radial glia: The consequences of asymmetric inheritance. Current Opinion in Neurobiology. doi:10.1016/S0959-4388(03)00013-8

Fothergill, T., Donahoo, A. L., Douglass, A., Zalucki, O., Yuan, J., Shu, T., ... Richards, L. J. (2013). Netrin-DCC Signaling Regulates Corpus Callosum Formation Through Attraction of Pioneering Axons and by Modulating Slit2-Mediated Repulsion. Cereb Cortex. doi:bhs395 [pii] 10.1093/cercor/bhs395

Frantz, G. D., \& McConnell, S. K. (1996). Restriction of late cerebral cortical progenitors to an upper-layer fate. Neuron, 17, 55-61. doi:10.1016/S08966273(00)80280-9

Friedman, P. A., \& Platzer, E. G. (1978). Interaction of anthelmintic benzimidazoles and benzimidazole derivatives with bovine brain tubulin. Biochimica et Biophysica Acta, 544(3), 605-14.

Galjart, N. (2005). CLIPs and CLASPs and cellular dynamics. Nature Reviews. Molecular Cell Biology, 6(6), 487-98. doi:10.1038/nrm1664

Gallo, G. (2011). The cytoskeletal and signaling mechanisms of axon collateral branching. Developmental Neurobiology, 71(3), 201-20. doi:10.1002/dneu.20852

Garavelli, L., \& Mainardi, P. C. (2007). Mowat-Wilson syndrome. Orphanet Journal of Rare Diseases, 2, 42. doi:10.1186/1750-1172-2-42

Garcez, P. P., Henrique, N. P., Furtado, D. A., Bolz, J., Lent, R., \& Uziel, D. (2007). Axons of callosal neurons bifurcate transiently at the white matter before consolidating an interhemispheric projection. Eur J Neurosci, 25(5), 1384-1394. doi:EJN5387 [pii] 10.1111/j.1460-9568.2007.05387.x 
Geschwind, D. H., \& Rakic, P. (2013). Perspective Cortical Evolution : Judge the Brain by Its Cover. Neuron, 633-647.

Gitai, Z., Yu, T. W., Lundquist, E. A., Tessier-Lavigne, M., \& Bargmann, C. I. (2003). The netrin receptor UNC-40/DCC stimulates axon attraction and outgrowth through enabled and, in parallel, Rac and UNC-115/ AbLIM. Neuron, 37(1), 53-65. doi:S0896627302011492 [pii]

Goebbels, S., Bormuth, I., Bode, U., Hermanson, O., Schwab, M. H., \& Nave, K.-A. (2006). Genetic targeting of principal neurons in neocortex and hippocampus of NEX-Cre mice. Genesis (New York, N.Y. : 2000), 44, 611621. doi:17146780

Goldberg, J. L. (2003). How does an axon grow? Genes \& Development, 17(8), 941-58. doi:10.1101/gad.1062303

Goodman, C. S., \& Shatz, C. J. (1993). Developmental mechanisms that generate precise patterns of neuronal connectivity. Cell, 72 Suppl, 77-98.

Grabitz, A. L., \& Duncan, M. K. (2012). Focus on molecules: Smad Interacting Protein 1 (Sip1, ZEB2, ZFHX1B). Experimental Eye Research, 101, 105-6. doi:10.1016/j.exer.2010.09.010

Greig, L. C., Woodworth, M. B., Galazo, M. J., Padmanabhan, H., \& Macklis, J. D. (2013). Molecular logic of neocortical projection neuron specification, development and diversity. Nature Reviews. Neuroscience, 14(11), 755-769. doi:10.1038/nrn3586

Grooteclaes, M. L., \& Frisch, S. M. (2000). Evidence for a function of CtBP in epithelial gene regulation and anoikis. Oncogene, 19(33), 3823-8. doi:10.1038/sj.onc.1203721

Guo, C., Eckler, M. J., McKenna, W. L., McKinsey, G. L., Rubenstein, J. L. R., \& Chen, B. (2013). Fezf2 expression identifies a multipotent progenitor for neocortical projection neurons, astrocytes, and oligodendrocytes. Neuron, 80(5), 1167-1174. doi:10.1016/j.neuron.2013.09.037

Hamasaki, T., Goto, S., Nishikawa, S., \& Ushio, Y. (2001). A role of netrin-1 in the formation of the subcortical structure striatum: repulsive action on the migration of late-born striatal neurons. The Journal of Neuroscience : The Official Journal of the Society for Neuroscience, 21(12), 4272-80.

Hamelin, M., Zhou, Y., Su, M. W., Scott, I. M., \& Culotti, J. G. (1993). Expression of the UNC-5 guidance receptor in the touch neurons of C. elegans steers their axons dorsally. Nature, 364(6435), 327-330. doi:10.1038/364327a0

Han, W., Kwan, K. Y., Shim, S., Lam, M. M. S., Shin, Y., Xu, X., ... Sestan, N. (2011). TBR1 directly represses Fezf2 to control the laminar origin and development of the corticospinal tract. Proceedings of the National Academy of Sciences of the United States of America, 108(7), 3041-6. doi:10.1073/pnas.1016723108

Harwell, C. C., Parker, P. R. L., Gee, S. M., Okada, A., McConnell, S. K., Kreitzer, A. C., \& Kriegstein, A. R. (2012). Sonic hedgehog expression in corticofugal projection neurons directs cortical microcircuit formation. Neuron, 73(6), 1116-26. doi:10.1016/j.neuron.2012.02.009

Haubensak, W., Attardo, A., Denk, W., \& Huttner, W. B. (2004). Neurons arise in the basal neuroepithelium of the early mammalian telencephalon: a major site of neurogenesis. Proceedings of the National Academy of Sciences 
of the United States of America, 101, 3196-3201.

doi:10.1073/pnas.0308600100

Hedgecock, E. M., Culotti, J. G., \& Hall, D. H. (1990). The unc-5, unc-6, and unc-40 genes guide circumferential migrations of pioneer axons and mesodermal cells on the epidermis in C. elegans. Neuron, 4(1), 61-85. doi:0896-6273(90)90444-K [pii]

Heffner, C. D., Lumsden, A. G., \& O'Leary, D. D. (1990). Target control of collateral extension and directional axon growth in the mammalian brain. Science (New York, N.Y.), 247(4939), 217-20.

Heidemann, S. R., Landers, J. M., \& Hamborg, M. A. (1981). Polarity orientation of axonal microtubules. The Journal of Cell Biology, 91, 661-665. doi:10.1083/jcb.91.3.661

Heldin, C. H., Miyazono, K., \& ten Dijke, P. (1997). TGF-beta signalling from cell membrane to nucleus through SMAD proteins. Nature, 390(6659), 465-71. doi:10.1038/37284

Hevner, R. F., Miyashita-Lin, E., \& Rubenstein, J. L. R. (2002). Cortical and thalamic axon pathfinding defects in Tbr1, Gbx2, and Pax6 mutant mice: evidence that cortical and thalamic axons interact and guide each other. The Journal of Comparative Neurology, 447(1), 8-17. doi:10.1002/cne.10219

Higashi, Y., Maruhashi, M., Nelles, L., Putte, T. Van De, Verschueren, K., Miyoshi, T., ... Huylebroeck, D. (2002). Generation of the Floxed Allele of the SIP1 ( Smad-Interacting Protein 1 ) Gene for Cre-Mediated Conditional Knockout in the Mouse. Genesis, 32, 82-84. doi:10.1002/gene.10048

Hill, R. S., \& Walsh, C. A. (2005). Molecular insights into human brain evolution. Nature, 437(7055), 64-67. doi:nature04103 [pii] 10.1038 / nature04103

Hoebeke, J., Van Nijen, G., \& De Brabander, M. (1976). Interaction of oncodazole (R 17934), a new antitumoral drug, with rat brain tubulin. Biochemical and Biophysical Research Communications, 69(2), 319-24.

Hong, K., Hinck, L., Nishiyama, M., Poo, M. M., Tessier-Lavigne, M., \& Stein, E. (1999). A ligand-gated association between cytoplasmic domains of UNC5 and DCC family receptors converts netrin-induced growth cone attraction to repulsion. Cell, 97(7), 927-941. doi:S0092-8674(00)80804-1 [pii]

Hong, Y. R., Chen, C. H., Chang, J. H., Wang, S., Sy, W. D., Chou, C. K., \& Howng, S. L. (2000). Cloning and characterization of a novel human ninein protein that interacts with the glycogen synthase kinase 3 beta. Biochimica et Biophysica Acta, 1492, 513-516.

Huang, Z. (2009). Molecular regulation of neuronal migration during neocortical development. Molecular and Cellular Neurosciences, 42(1), 1122. doi:10.1016/j.mcn.2009.06.003

Hur, E.-M., Saijilafu, Lee, B. D., Kim, S.-J., Xu, W.-L., \& Zhou, F.-Q. (2011). GSK3 controls axon growth via CLASP-mediated regulation of growth cone microtubules. Genes \& Development, 25(18), 1968-81.

doi:10.1101/gad.17015911 
Innocenti, G. M., \& Price, D. J. (2005). Exuberance in the development of cortical networks. Nature Reviews. Neuroscience, 6(12), 955-65. doi:10.1038/nrn1790

Inoué, S., \& Salmon, E. D. (1995). Force generation by microtubule assembly/disassembly in mitosis and related movements. Molecular Biology of the Cell, 6(12), 1619-40.

Ireland, C. M., Gull, K., Gutteridge, W. E., \& Pogson, C. I. (1979). The interaction of benzimidazole carbamates with mammalian microtobule protein. Biochemical Pharmacology, 28(17), 2680-2.

Ishii, N., Wadsworth, W. G., Stern, B. D., Culotti, J. G., \& Hedgecock, E. M. (1992). UNC-6, a laminin-related protein, guides cell and pioneer axon migrations in C. elegans. Neuron, 9(5), 873-881. doi:0896-6273(92)90240-E [pii]

Islam, S. M., Shinmyo, Y., Okafuji, T., Su, Y., Naser, I. B., Ahmed, G., ... Tanaka, H. (2009). Draxin, a repulsive guidance protein for spinal cord and forebrain commissures. Science, 323(5912), 388-393. doi:10.1126/science.1165187 323/5912/388 [pii]

Ivy, G. O., \& Killackey, H. P. (1981). The ontogeny of the distribution of callosal projection neurons in the rat parietal cortex. The Journal of Comparative Neurology, 195(3), 367-89. doi:10.1002/cne.901950302

Jeub, M., Emrich, M., Pradier, B., Taha, O., Gailus-Durner, V., Fuchs, H., ... Racz, I. (2011). The transcription factor Smad-interacting protein 1 controls pain sensitivity via modulation of DRG neuron excitability. Pain, 152(10), 2384-98. doi:10.1016/j.pain.2011.07.006

Kalil, K., Szebenyi, G., \& Dent, E. W. (2000). Common Mechanisms Underlying Growth Cone Guidance and Axon Branching.

Kaprielian, Z., Imondi, R., \& Runko, E. (2000). Axon guidance at the midline of the developing CNS. The Anatomical Record, 261(5), 176-97.

Keating, T. J., \& Borisy, G. G. (1999). Centrosomal and non-centrosomal microtubules. Biology of the Cell / under the Auspices of the European Cell Biology Organization, 91(4-5), 321-9.

Keating, T. J., Peloquin, J. G., Rodionov, V. I., Momcilovic, D., \& Borisy, G. G. (1997). Microtubule release from the centrosome. Proceedings of the National Academy of Sciences of the United States of America, 94(10), 5078-83.

Keino-Masu, K., Masu, M., Hinck, L., Leonardo, E. D., Chan, S. S., Culotti, J. G., \& Tessier-Lavigne, M. (1996). Deleted in Colorectal Cancer (DCC) encodes a netrin receptor. Cell, 87(2), 175-185. doi:S0092-8674(00)81336-7 [pii]

Kellogg, D., Moritz, M., \& Alberts, B. (1994). THE CENTROSOME AND CELLULAR ORGANIZATION. Annual Review of ....

Kennedy, H., \& Dehay, C. (1993). Cortical specification of mice and men. Cerebral Cortex (New York, N.Y. : 1991), 3(3), 171-86.

Kennedy, T. E., Serafini, T., de la Torre, J. R., \& Tessier-Lavigne, M. (1994). Netrins are diffusible chemotropic factors for commissural axons in the embryonic spinal cord. Cell, 78, 425-435. doi:10.1016/0092-8674(94)904219

Kennedy, T. E., \& Tessier-Lavigne, M. (1995). Guidance and induction of branch formation in developing axons by target-derived diffusible 
factors. Current Opinion in Neurobiology, 5, 83-90. doi:10.1016/09594388(95)80091-3

Kidd, T., Brose, K., Mitchell, K. J., Fetter, R. D., Tessier-Lavigne, M., Goodman, C. S., \& Tear, G. (1998). Roundabout controls axon crossing of the CNS midline and defines a novel subfamily of evolutionarily conserved guidance receptors. Cell, 92, 205-215. doi:10.1016/S00928674(00)80915-0

Killeen, M. T., \& Sybingco, S. S. (2008). Netrin, Slit and Wnt receptors allow axons to choose the axis of migration. Developmental Biology, 323, 143-151. doi:10.1016/j.ydbio.2008.08.027

Kim, D., \& Ackerman, S. L. (2011). The UNC5C netrin receptor regulates dorsal guidance of mouse hindbrain axons. J Neurosci, 31(6), 2167-2179. doi:31/6/2167 [pii] 10.1523/JNEUROSCI.5254-10.2011

Kim, G. J., Shatz, C. J., \& McConnell, S. K. (1991). Morphology of pioneer and follower growth cones in the developing cerebral cortex. Journal of Neurobiology, 22, 629-642.

Kirschner, M., \& Mitchison, T. (1986). Beyond self-assembly: from microtubules to morphogenesis. Cell, 45, 329-342. doi:10.1016/00928674(86)90318-1

Klämbt, C., Jacobs, J. R., \& Goodman, C. S. (1991). The midline of the drosophila central nervous system: A model for the genetic analysis of cell fate, cell migration, and growth cone guidance. Cell, 64(4), 801-815. doi:10.1016/0092-8674(91)90509-W

Kmet, M., Guo, C., Edmondson, C., \& Chen, B. (2013). Directed differentiation of human embryonic stem cells into corticofugal neurons uncovers heterogeneous Fezf2-expressing subpopulations. PloS One, 8, e67292. doi:10.1371/journal.pone.0067292

Koester, E., \& O'Leary, D. (1994). Axons of Early Generated Corpus Callosum Neurons in Cingulate Cortex Pioneer the. The Journal of Neuroscience, 14(November).

Kretzschmar, M., \& Massague, J. (1998). SMADs : mediators and regulators of TGF-13 signaling I III. Current Opinion in Genetics \& Development, 8, 103-111.

Kriegstein, A. R., \& Noctor, S. C. (2004). Patterns of neuronal migration in the embryonic cortex. Trends in Neurosciences, 27(7), 392-399. doi:10.1016/j.tins.2004.05.001

Kritzer, M. F., \& Goldman-Rakic, P. S. (1995). Intrinsic circuit organization of the major layers and sublayers of the dorsolateral prefrontal cortex in the rhesus monkey. The Journal of Comparative Neurology, 359(1), 131-43. doi:10.1002/cne.903590109

Kuijpers, M., \& Hoogenraad, C. C. (2011). Centrosomes, microtubules and neuronal development. Molecular and Cellular Neurosciences, 48(4), 349-58. doi:10.1016/j.mcn.2011.05.004

Kullander, K., \& Klein, R. (2002). Mechanisms and functions of Eph and ephrin signalling. Nature Reviews. Molecular Cell Biology, 3, 475-486. doi:10.1038/nrm856

Kwan, K. Y., Lam, M. M. S., Krsnik, Z., Kawasawa, Y. I., Lefebvre, V., \& Sestan, N. (2008). SOX5 postmitotically regulates migration, 
postmigratory differentiation, and projections of subplate and deep-layer neocortical neurons. Proceedings of the National Academy of Sciences of the United States of America, 105(41), 16021-6. doi:10.1073/pnas.0806791105

Lai, T., Jabaudon, D., Molyneaux, B. J., Azim, E., Arlotta, P., Menezes, J. R. L., \& Macklis, J. D. (2008). SOX5 controls the sequential generation of distinct corticofugal neuron subtypes. Neuron, 57(2), 232-47. doi:10.1016/j.neuron.2007.12.023

Lee, J. C., Field, D. J., \& Lee, L. L. (1980). Effects of nocodazole on structures of calf brain tubulin. Biochemistry, 19(26), 6209-15.

Lickiss, T., Cheung, A. F., Hutchinson, C. E., Taylor, J. S., \& Molnar, Z. (2012). Examining the relationship between early axon growth and transcription factor expression in the developing cerebral cortex. J Anat, 220(3), 201211. doi:10.1111/j.1469-7580.2011.01466.x

Long, H., Sabatier, C., Ma, L., Plump, A., Yuan, W., Ornitz, D. M., ... TessierLavigne, M. (2004). Conserved roles for Slit and Robo proteins in midline commissural axon guidance. Neuron, 42, 213-223. doi:10.1016/S08966273(04)00179-5

Malatesta, P., Hartfuss, E., \& Götz, M. (2000a). Isolation of radial glial cells by fluorescent-activated cell sorting reveals a neuronal lineage. Development (Cambridge, England), 127(24), 5253-5263.

Malatesta, P., Hartfuss, E., \& Götz, M. (2000b). Isolation of radial glial cells by fluorescent-activated cell sorting reveals a neuronal lineage. Development (Cambridge, England), 127(24), 5253-5263.

McConnell, S. K. (1995). Constructing the cerebral cortex: neurogenesis and fate determination. Neuron, 15(4), 761-768.

McConnell, S. K., Ghosh, A., \& Shatz, C. J. (1989). Subplate neurons pioneer the first axon pathway from the cerebral cortex. Science (New York, N.Y.), 245, 978-982. doi:10.1126/ science.2475909

McKenna, W. L., Betancourt, J., Larkin, K. A., Abrams, B., Guo, C., Rubenstein, J. L. R., \& Chen, B. (2011). Tbr1 and Fezf2 regulate alternate corticofugal neuronal identities during neocortical development. The Journal of Neuroscience : The Official Journal of the Society for Neuroscience, 31(2), 549-64. doi:10.1523/JNEUROSCI.4131-10.2011

McKinsey, G. L., Lindtner, S., Trzcinski, B., Visel, A., Pennacchio, L. a, Huylebroeck, D., ... Rubenstein, J. L. R. (2013). Dlx1\&2-dependent expression of Zfhx1b (Sip1, Zeb2) regulates the fate switch between cortical and striatal interneurons. Neuron, 77(1), 83-98. doi:10.1016/j.neuron.2012.11.035

Metin, C., Deleglise, D., Serafini, T., Kennedy, T. E., \& Tessier-Lavigne, M. (1997). A role for netrin-1 in the guidance of cortical efferents. Development, 124(24), 5063-5074.

Métin, C., Deléglise, D., Serafini, T., Kennedy, T. E., \& Tessier-Lavigne, M. (1997). A role for netrin-1 in the guidance of cortical efferents. Development (Cambridge, England), 124(24), 5063-74.

Miquelajauregui, A., Van de Putte, T., Polyakov, A., Nityanandam, A., Boppana, S., Seuntjens, E., ... Tarabykin, V. (2007). Smad-interacting protein-1 (Zfhx1b) acts upstream of Wnt signaling in the mouse 
hippocampus and controls its formation. Proceedings of the ..., 104(31), 12919-12924.

Mitchison, T., \& Kirschner, M. (1984). Dynamic instability of microtubule growth. Nature, 312(5991), 237-42.

Miyata, T., Kawaguchi, a, Okano, H., \& Ogawa, M. (2001). Asymmetric inheritance of radial glial fibers by cortical neurons. Neuron, 31(5), 727741.

Moal, F. D., Wilson, M., Mowat, D., Collot, N., Niel, F., \& Ã, M. G. (2007). ZFHX1B Mutations in Patients With Mowat- Wilson Syndrome, 28(4), 313-321. doi:10.1002/humu

Mogensen, M. M. (1999). Microtubule release and capture in epithelial cells. Biology of the Cell / under the Auspices of the European Cell Biology Organization, 91(4-5), 331-41.

Mogensen, M. M., Malik, a, Piel, M., Bouckson-Castaing, V., \& Bornens, M. (2000). Microtubule minus-end anchorage at centrosomal and noncentrosomal sites: the role of ninein. Journal of Cell Science, 113 ( Pt 1, 3013-23.

Molnar, Z., Adams, R., \& Blakemore, C. (1998). Mechanisms Underlying the Early Establishment of Thalamocortical Connections in the Rat. J. Neurosci., 18(15), 5723-5745.

Molnár, Z., Hoerder-Suabedissen, A., Wang, W. Z., DeProto, J., Davies, K., Lee, S., ... Cheung, A. F. P. (2007). Genes involved in the formation of the earliest cortical circuits. Novartis Foundation Symposium, 288, 212-24; discussion 224-9, 276-81.

Molyneaux, B. J., Arlotta, P., Fame, R. M., MacDonald, J. L., MacQuarrie, K. L., \& Macklis, J. D. (2009). Novel subtype-specific genes identify distinct subpopulations of callosal projection neurons. J Neurosci, 29(39), 1234312354. doi:10.1523/JNEUROSCI.6108-08.2009 29/39/12343 [pii]

Molyneaux, B. J., Arlotta, P., Menezes, J. R. L., \& Macklis, J. D. (2007). Neuronal subtype specification in the cerebral cortex. Nature Reviews. Neuroscience, 8(6), 427-37. doi:10.1038/nrn2151

Mortimer, D., Feldner, J., Vaughan, T., Vetter, I., Pujic, Z., Rosoff, W. J., ... Goodhill, G. J. (2009). Bayesian model predicts the response of axons to molecular gradients. Proc Natl Acad Sci U S A, 106(25), 10296-10301. doi:10.1073/pnas.0900715106 0900715106 [pii]

Moss, D. K., Bellett, G., Carter, J. M., Liovic, M., Keynton, J., Prescott, A. R., ... Mogensen, M. M. (2007). Ninein is released from the centrosome and moves bi-directionally along microtubules. Journal of Cell Science, $120(\mathrm{Pt}$ 17), 3064-74. doi:10.1242/jcs.010322

Muramatsu, R., Nakahara, S., Ichikawa, J., Watanabe, K., Matsuki, N., \& Koyama, R. (2010). The ratio of "deleted in colorectal cancer" to "uncoordinated-5A" netrin-1 receptors on the growth cone regulates mossy fibre directionality. Brain, 133(Pt 1), 60-75. doi:awp266 [pii] 10.1093/brain/awp266

Murphy, D. B., Vallee, R. B., \& Borisy, G. G. (1977). Identity and polymerization-stimulatory activity of the nontubulin proteins associated with microtubules. Biochemistry, 16(12), 2598-605. 
Nadarajah, B., Brunstrom, J. E., Grutzendler, J., Wong, R. O., \& Pearlman, a L. (2001). Two modes of radial migration in early development of the cerebral cortex. Nature Neuroscience, 4(2), 143-50. doi:10.1038/83967

Nadarajah, B., \& Parnavelas, J. G. (2002). Modes of neuronal migration in the developing cerebral cortex. Nature Reviews. Neuroscience, 3(6), 423-32. doi:10.1038/nrn845

Nakamura, F., Kalb, R. G., \& Strittmatter, S. M. (2000). Molecular basis of semaphorin-mediated axon guidance. Journal of Neurobiology, 44, 219-229. doi:10.1002/1097-4695(200008)44:2<219::AID-NEU11>3.0.CO;2-W

Neukirchen, D., \& Bradke, F. (2011). Neuronal polarization and the cytoskeleton. Seminars in Cell E Developmental Biology, 22(8), 825-33. doi:10.1016/j.semcdb.2011.08.007

Niquille, M., Garel, S., Mann, F., Hornung, J.-P., Otsmane, B., Chevalley, S., ... Lebrand, C. (2009). Transient neuronal populations are required to guide callosal axons: a role for semaphorin 3C. PLoS Biology, 7(10), e1000230. doi:10.1371/journal.pbio.1000230

Noctor, S. C., Flint, a C., Weissman, T. a, Dammerman, R. S., \& Kriegstein, a R. (2001). Neurons derived from radial glial cells establish radial units in neocortex. Nature, 409(6821), 714-720. doi:10.1038/35055553

Noctor, S. C., Flint, A. C., Weissman, T. a, Wong, W. S., Clinton, B. K., \& Kriegstein, A. R. (2002). Dividing precursor cells of the embryonic cortical ventricular zone have morphological and molecular characteristics of radial glia. The Journal of Neuroscience : The Official Journal of the Society for Neuroscience, 22(8), 3161-3173. doi:20026299

Noctor, S. C., Martínez-Cerdeño, V., Ivic, L., \& Kriegstein, A. R. (2004). Cortical neurons arise in symmetric and asymmetric division zones and migrate through specific phases. Nature Neuroscience, 7(2), 136-144. doi:10.1038/nn1172

O'Leary, D. M. (1996). Dynamics of Target Recognition by Interstitial along Developing Cortical Axons Axon Branching, 76(4), 1450-1459.

O'Leary, D. M., \& Terashima, T. (1988). Cortical axons branch to multiple subcortical targets by interstitial axon budding: implications for target recognition and "waiting periods". Neuron, 1(10), 901-10.

Ohama, Y., \& Hayashi, K. (2009). Relocalization of a microtubule-anchoring protein, ninein, from the centrosome to dendrites during differentiation of mouse neurons. Histochemistry and Cell Biology, 132(5), 515-24. doi:10.1007/s00418-009-0631-z

Paul, L. K., Brown, W. S., Adolphs, R., Tyszka, J. M., Richards, L. J., Mukherjee, P., \& Sherr, E. H. (2007). Agenesis of the corpus callosum: genetic, developmental and functional aspects of connectivity. Nature Reviews. Neuroscience, 8(4), 287-99. doi:10.1038/nrn2107

Piel, M., Meyer, P., Khodjakov, A., Rieder, C. L., \& Bornens, M. (2000). The respective contributions of the mother and daughter centrioles to centrosome activity and behavior in vertebrate cells. The Journal of Cell Biology, 149(2), 317-30.

Polleux, F., \& Ghosh, A. (2002). The slice overlay assay: a versatile tool to study the influence of extracellular signals on neuronal development. Sci STKE, 2002(136), p19. doi:10.1126/stke.2002.136.p19 2002/136/p19 [pii] 
Portera-Cailliau, C., Weimer, R. M., De Paola, V., Caroni, P., \& Svoboda, K. (2005). Diverse modes of axon elaboration in the developing neocortex. PLoS Biology, 3(8), e272. doi:10.1371/journal.pbio.0030272

Postigo, A. a. (2003). Opposing functions of ZEB proteins in the regulation of the TGFbeta/BMP signaling pathway. The EMBO Journal, 22(10), 2443-52. doi:10.1093/emboj/cdg225

Postigo, A. a, \& Dean, D. C. (2000). Differential expression and function of members of the zfh-1 family of zinc finger/homeodomain repressors. Proceedings of the National Academy of Sciences of the United States of America, 97(12), 6391-6.

Postigo, A. a, Depp, J. L., Taylor, J. J., \& Kroll, K. L. (2003). Regulation of Smad signaling through a differential recruitment of coactivators and corepressors by ZEB proteins. The EMBO Journal, 22(10), 2453-62. doi:10.1093/emboj/cdg226

Quinn, C. C., \& Wadsworth, W. G. (2008). Axon guidance: asymmetric signaling orients polarized outgrowth. Trends in Cell Biology, 18(12), 597603. doi:10.1016/j.tcb.2008.09.005

Rakic, P. (1974). Neurons in rhesus monkey visual cortex: systematic relation between time of origin and eventual disposition. Science (New York, N.Y.), 183, 425-427. doi:10.1126/ science.183.4123.425

Rash, B. G., \& Richards, L. J. (2001). A role for cingulate pioneering axons in the development of the corpus callosum. The Journal of Comparative Neurology, 434(2), 147-57.

Remacle, J. E., Kraft, H., Lerchner, W., Wuytens, G., Collart, C., Verschueren, K., ... Huylebroeck, D. (1999). New mode of DNA binding of multi-zinc finger transcription factors: deltaEF1 family members bind with two hands to two target sites. The EMBO Journal, 18(18), 5073-84. doi:10.1093/emboj/18.18.5073

Ren, T., Zhang, J., Plachez, C., Mori, S., \& Richards, L. J. (2007). Diffusion tensor magnetic resonance imaging and tract-tracing analysis of Probst bundle structure in Netrin1- and DCC-deficient mice. J Neurosci, 27(39), 10345-10349. doi:27/39/10345 [pii] 10.1523/JNEUROSCI.2787-07.2007

Richards, L. J., Plachez, C., \& Ren, T. (2004). Mechanisms regulating the development of the corpus callosum and its agenesis in mouse and human. Clinical Genetics, 66(4), 276-89. doi:10.1111/j.13990004.2004.00354.x

Robin, M. (2006). The Corpus Callosum as an Evolutionary Innovation. JOURNAL OF EXPERIMENTAL ZOOLOGY, 17(August 2005), 8-17. doi:10.1002/jez.b

Rodionov, V., Nadezhdina, E., \& Borisy, G. (1999). Centrosomal control of microtubule dynamics. Proceedings of the National Academy of Sciences of the United States of America, 96(January), 115-120.

Rouaux, C., \& Arlotta, P. (2013). Direct lineage reprogramming of post-mitotic callosal neurons into corticofugal neurons in vivo. Nature Cell Biology, 15(2), 214-221. doi:10.1038/ncb2660

Sabatier, C., Plump, A. S., Brose, K., Tamada, A., Murakami, F., Lee, E. Y.-H. ., \& Tessier-Lavigne, M. (2004). The Divergent Robo Family Protein Rig1/Robo3 Is a Negative Regulator of Slit Responsiveness Required for 
Midline Crossing by Commissural Axons. Cell, 117(2), 157-169. doi:10.1016/S0092-8674(04)00303-4

Saito, T. (2006). In vivo electroporation in the embryonic mouse central nervous system. Nat Protoc, 1(3), 1552-1558. doi:nprot.2006.276 [pii] 10.1038/nprot.2006.276

Sakakibara, A., Ando, R., Sapir, T., \& Tanaka, T. (2013). Microtubule dynamics in neuronal morphogenesis. Open Biology, 3(7), 130061. doi:10.1098/rsob.130061

Schmidt, H., \& Rathjen, F. G. (2010). Signalling mechanisms regulating axonal branching in vivo. BioEssays : News and Reviews in Molecular, Cellular and Developmental Biology, 32(11), 977-85. doi:10.1002/bies.201000054

Schüz, A., \& Preiß1, H. (1996). Basic Connectivity of the Cerebral Cortex and some Considerations on the Corpus Callosum. Neuroscience $\mathcal{E}$ Biobehavioral Reviews, 20(4), 567-570. doi:10.1016/0149-7634(95)00069-0

Seeger, M., Tear, G., Ferres-Marco, D., \& Goodman, C. S. (1993). Mutations affecting growth cone guidance in Drosophila: genes necessary for guidance toward or away from the midline. Neuron, 10, 409-426. doi:10.1016/0896-6273(93)90330-T

Serafini, T., Colamarino, S. A., Leonardo, E. D., Wang, H., Beddington, R., Skarnes, W. C., \& Tessier-Lavigne, M. (1996). Netrin-1 is required for commissural axon guidance in the developing vertebrate nervous system. Cell, 87, 1001-1014. doi:10.1016/S0092-8674(00)81795-X

Seuntjens, E., Nityanandam, A., Miquelajauregui, A., Debruyn, J., Stryjewska, A., Goebbels, S., ... Tarabykin, V. (2009). Sip1 regulates sequential fate decisions by feedback signaling from postmitotic neurons to progenitors. Nat Neurosci, 12(11), 1373-1380. doi:nn.2409 [pii] 10.1038/nn.2409

Sharp, D. J., \& Ross, J. L. (2012). Microtubule-severing enzymes at the cutting edge. Journal of Cell Science, 125(Pt 11), 2561-9. doi:10.1242/jcs.101139

Shinohara, H., Sakayori, N., Takahashi, M., \& Osumi, N. (2013). Ninein is essential for the maintenance of the cortical progenitor character by anchoring the centrosome to microtubules. Biology Open, 2(7), 739-49. doi:10.1242/ bio.20135231

Shu, T., Puche, A. C., \& Richards, L. J. (2003). Development of midline glial populations at the corticoseptal boundary. Journal of Neurobiology, 57(1), 81-94. doi:10.1002/neu.10252

Shu, T., \& Richards, L. J. (2001). Cortical axon guidance by the glial wedge during the development of the corpus callosum. The Journal of Neuroscience : The Official Journal of the Society for Neuroscience, 21(8), 2749-58.

Shu, T., Ying, L., Keller, A., \& Richards, L. J. (2003). The glial sling is a migratory population of developing neurons. Development, 130(13), 29292937. doi:10.1242/dev.00514

Silver, J., Edwards, M. a, \& Levitt, P. (1993). Immunocytochemical demonstration of early appearing astroglial structures that form boundaries and pathways along axon tracts in the fetal brain. The Journal of Comparative Neurology, 328(3), 415-36. doi:10.1002/cne.903280308 
Silver, J., \& Ogawa, M. Y. (1983). Postnatally induced formation of the corpus callosum in acallosal mice on glia-coated cellulose bridges. Science (New York, N.Y.), 220(4601), 1067-9.

Smith, K. M., Ohkubo, Y., Maragnoli, M. E., Rasin, M.-R., Schwartz, M. L., Sestan, N., \& Vaccarino, F. M. (2006). Midline radial glia translocation and corpus callosum formation require FGF signaling. Nature Neuroscience, 9(6), 787-97. doi:10.1038/nn1705

Srinivasan, K., Leone, D. P., Bateson, R. K., Dobreva, G., Kohwi, Y., KohwiShigematsu, T., ... McConnell, S. K. (2012). A network of genetic repression and derepression specifies projection fates in the developing neocortex. Proc Natl Acad Sci U S A, 109(47), 19071-19078. doi:10.1073/pnas.1216793109 1216793109 [pii]

Stearns, T., \& Kirschner, M. (1994). In vitro reconstitution of centrosome assembly and function: the central role of gamma-tubulin. Cell, 76(4), 623-37.

Stepanova, T., Slemmer, J., Hoogenraad, C. C., Lansbergen, G., Dortland, B., De Zeeuw, C. I., ... Galjart, N. (2003). Visualization of microtubule growth in cultured neurons via the use of EB3-GFP (end-binding protein 3-green fluorescent protein). The Journal of Neuroscience : The Official Journal of the Society for Neuroscience, 23(7), 2655-64.

Szebenyi, G., Callaway, J. L., Dent, E. W., \& Kalil, K. (1998). Interstitial branches develop from active regions of the axon demarcated by the primary growth cone during pausing behaviors. The Journal of Neuroscience : The Official Journal of the Society for Neuroscience, 18(19), 7930-40.

Szemes, M., Gyorgy, A., Paweletz, C., Dobi, A., \& Agoston, D. V. (2006). Isolation and characterization of SATB2, a novel AT-rich DNA binding protein expressed in development- and cell-specific manner in the rat brain. Neurochem Res, 31(2), 237-246. doi:10.1007/s11064-005-9012-8

Takahashi, T. (1995). The Cell Cycle of the Pseudostratified Embryonic Murine Cerebral Wall Ventricular Epithelium of the, 15(September), 6046-6057.

Tarabykin, V., Stoykova, A., Usman, N., \& Gruss, P. (2001). Cortical upper layer neurons derive from the subventricular zone as indicated by Svet1 gene expression. Development (Cambridge, England), 128, 1983-1993.

Tessier-Lavigne, M., \& Goodman, C. S. (1996). The Molecular Biology of Axon Guidance. Science. doi:10.1126/science.274.5290.1123

Thomson, A. M., \& Lamy, C. (2007). Functional maps of neocortical local circuitry EMPLOYED TO STUDY LOCAL CIRCUIT, 1(1).

Van de Putte, T., Maruhashi, M., Francis, A., Nelles, L., Kondoh, H., Huylebroeck, D., \& Higashi, Y. (2003). Mice lacking ZFHX1B, the gene that codes for Smad-interacting protein-1, reveal a role for multiple neural crest cell defects in the etiology of Hirschsprung disease-mental retardation syndrome. American Journal of Human Genetics, 72(2), 465-70. doi:10.1086/346092

Van den Berghe, V., Stappers, E., Vandesande, B., Dimidschstein, J., Kroes, R., Francis, A., ... Seuntjens, E. (2013). Directed migration of cortical 
interneurons depends on the cell-autonomous action of Sip1. Neuron, 77(1), 70-82. doi:10.1016/j.neuron.2012.11.009

Van Grunsven, L. A., Papin, C., Avalosse, B., Opdecamp, K., Huylebroeck, D., Smith, J. C., \& Bellefroid, E. J. (2000). XSIP1, a Xenopus zinc finger/homeodomain encoding gene highly expressed during early neural development. Mechanisms of Development, 94, 189-193. doi:10.1016/S0925-4773(00)00318-X

Vasquez, R. J., Howell, B., Yvon, a M., Wadsworth, P., \& Cassimeris, L. (1997). Nanomolar concentrations of nocodazole alter microtubule dynamic instability in vivo and in vitro. Molecular Biology of the Cell, 8(6), 973-85.

Verschueren, K. (1999). SIP1, a Novel Zinc Finger/Homeodomain Repressor, Interacts with Smad Proteins and Binds to 5'-CACCT Sequences in Candidate Target Genes. Journal of Biological Chemistry, 274(29), 2048920498. doi:10.1074/jbc.274.29.20489

Verstappen, G., van Grunsven, L. a, Michiels, C., Van de Putte, T., Souopgui, J., Van Damme, J., ... Huylebroeck, D. (2008). Atypical Mowat-Wilson patient confirms the importance of the novel association between ZFHX1B/SIP1 and NuRD corepressor complex. Human Molecular Genetics, 17(8), 1175-83. doi:10.1093/hmg/ddn007

W.J.Rosoff M.A.Esrick, G.J.Goodhill, J.S.Urbach, R. M. (2004). Generating controlled Molecular Gradients in 3D gels. Wiley Periodicals. doi:10.1002/ bit.20564

Wadsworth, W. G., Bhatt, H., \& Hedgecock, E. M. (1996). Neuroglia and pioneer neurons express UNC-6 to provide global and local netrin cues for guiding migrations in C. elegans. Neuron, 16(1), 35-46. doi:S08966273(00)80021-5 [pii]

Wakabayashi, Y., Watanabe, H., Inoue, J., Takeda, N., Sakata, J., Mishima, Y., ... Kominami, R. (2003). Bcl11b is required for differentiation and survival of alphabeta T lymphocytes. Nat Immunol, 4(6), 533-539. doi:10.1038/ni927 ni927 [pii]

Wakamatsu, N., Yamada, Y., Yamada, K., Ono, T., Nomura, N., Taniguchi, H., ... Nagaya, M. (2001). Mutations in SIP1, encoding Smad interacting protein-1, cause a form of Hirschsprung disease. Nature Genetics, 27(4), 369-70. doi:10.1038/86860

Walker, R. A., O'Brien, E. T., Pryer, N. K., Soboeiro, M. F., Voter, W. A., Erickson, H. P., \& D, S. E. (1988). Dynamic Instability of Individual Microtubules Analyzed by Video Light Microscopy: Rate Constants and Transition Frequencies, 107(October), 1437-1448.

Wang, X., Tsai, J., Imai, J., Lian, W., Vallee, R., \& Shi, S. (2009). Asymmetric centrosome inheritance maintains neural progenitors in neocortex. Nature, 461(7266), 947-955. doi:10.1038/nature08435.Asymmetric

Weimann, J. M., Zhang, Y. A., Levin, M. E., Devine, W. P., Brûlet, P., \& McConnell, S. K. (1999). Cortical Neurons Require Otx1 for the Refinement of Exuberant Axonal Projections to Subcortical Targets. Neuron, 24(4), 819-831. doi:10.1016/S0896-6273(00)81030-2

Weng, Q., Chen, Y., Wang, H., Xu, X., Yang, B., He, Q., ... Lu, Q. R. (2012). Dual-mode modulation of Smad signaling by Smad-interacting protein 
Sip1 is required for myelination in the central nervous system. Neuron, 73(4), 713-28. doi:10.1016/j.neuron.2011.12.021

Whitman, M. (1998). Smads and early developmental signaling by the TGFbeta superfamily. Genes \& Development, 12(16), 2445-2462. doi:10.1101/gad.12.16.2445

Wilson, M., Mowat, D., Dastot-Le Moal, F., Cacheux, V., Kääriäinen, H., Cass, D., ... Goossens, M. (2003). Further delineation of the phenotype associated with heterozygous mutations in ZFHX1B. American Journal of Medical Genetics. Part A, 119A(3), 257-65. doi:10.1002/ajmg.a.20053

Yamada, K. M., Spooner, B. S., \& Wessells, N. K. (1970). Axon growth: roles of microfilaments and microtubules. Proceedings of the National Academy of Sciences of the United States of America, 66(4), 1206-12.

Yamada, K., Yamada, Y., Nomura, N., Miura, K., Wakako, R., Hayakawa, C., ... Wakamatsu, N. (2001). Nonsense and frameshift mutations in ZFHX1B, encoding Smad-interacting protein 1, cause a complex developmental disorder with a great variety of clinical features. American Journal of Human Genetics, 69(6), 1178-85.

Yamamoto, N., Higashi, S., \& Toyama, K. (1997). Stop and branch behaviors of geniculocortical axons: a time-lapse study in organotypic cocultures. The Journal of Neuroscience : The Official Journal of the Society for Neuroscience, 17, 3653-3663.

Yoshimoto, A., Saigou, Y., Higashi, Y., \& Kondoh, H. (2005). Regulation of ocular lens development by Smad-interacting protein 1 involving Foxe3 activation. Development (Cambridge, England), 132(20), 4437-48. doi:10.1242/ dev.02022

Yu, W., Ahmad, F. J., \& Baas, P. W. (1994). Microtubule fragmentation and partitioning in the axon during collateral branch formation. The Journal of Neuroscience : The Official Journal of the Society for Neuroscience, 14, 58725884.

Yu, W., Centonze, V. E., Ahmad, F. J., \& Baas, P. W. (1993). Microtubule nucleation and release from the neuronal centrosome. The Journal of Cell Biology, 122(2), 349-59.

Yu, W., Qiang, L., Solowska, J. M., Karabay, A., Korulu, S., \& Baas, P. W. (2008). The microtubule-severing proteins spastin and katanin participate differently in the formation of axonal branches. Molecular Biology of the Cell, 19, 1485-1498. doi:10.1091/mbc.E07-09-0878

Zheng, Y., Wong, M. L., Alberts, B., \& Mitchison, T. (1995). Nucleation of microtubule assembly by a gamma-tubulin-containing ring complex. Nature, 378(6557), 578-83. doi:10.1038/378578a0 


\section{ACKNOWLEDGEMENTS}

I would like to thank my PhD supervisor Prof. Dr. Victor Tarabykin for taking me under his wing and for sharing his knowledge and providing valuable guidance through these past years. Victor, your energy has always been contagious and your enthusiasm for science will forever be an inspiration.

I would also like to extend my gratitude to all those who provided me with valuable reagents and resources that helped me advance my work, Manuela Schwark, Roman Wunderlich, Jutta Schüler, Denis Lajko and Marni Pollrich for their scientific and administrative assistance, Ingo Bormuth for unflinchingly dealing with all the bureaucratic paper work and animal licenses, Steffi and Ms. Stefanie Beyer and others who took care of my animal colonies at MPI-Göttingen and Charité-Berlin, and my friends and colleagues at the MPI-for Experimental Medicine, Göttingen, and the Institute for Cell and Neurobiology, Berlin for providing a delightful environment to work in.

I would also like to thank the International Max-Plank Research SchoolNeuroscience program especially Prof. Dr. Michael Hörner, Sandra Drübe, and my thesis committee members Dr. Judith Stegmüller and Prof. Dr. André Fischer for their continuous support and guidance during the course of my PhD.

A heartfelt thanks to the entire Boehringer Ingelheim Fonds team, with a special mention of Dr. Claudia Walther, Dr. Sabine Achten, Dr. Anja Hoffman and Sandra Schedler, for making my experience with the fellowship so personal and enjoyable, for believing in me, even when my confidence was running low and for connecting me to a whole world of opportunities and possibilities.

Thank you Mom, Dad, Ammamma and Thatha, none of this would be possible without your unconditional love and heartfelt blessings. You taught me to dream big and to believe that not even the sky is my limit. Thank you Ele, Johannes, Clementine, Theresa, Alejandro, Hema, Jatin, Anjana, Kruthika, Pratima, Neeta, Anu and all my other dear friends who have been my pillars of strength. Thank you for all the love and laughter! And lastly, and most importantly, thank you Srini, for being my home away from home. Your presence made work seem like play, turned failures into stepping-stones and made the taste of success seem that much sweeter. 